\title{
Indagine sul mercato \\ degli alloggi in locazione \\ nel comune di Bologna
}

Andrea Gentili

Filippo Tassinari

Andrea Zoboli

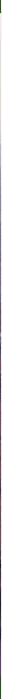


Fondazione di iricerca Istituto Carlo Cattaneo

\section{Indagine sul mercato \\ degli alloggi in locazione \\ nel comune di Bologna}

Andrea Gentili

Filippo Tassinari

Andrea Zoboli 


\section{Crediti}

Questa ricerca è l'esito del lavoro di indagine condotto dall'Istituto Carlo Cattaneo su incarico del Settore Politiche Abitative del Comune di Bologna. Le opinioni espresse in questo documento sono quelle degli autori e non rappresentano necessariamente quelle del committente.

Il gruppo di ricerca dell'Istituto Carlo Cattaneo:

PhD Andrea Gentili (responsabile) - Istituto Carlo Cattaneo, DSE Università di Bologna, DIMA Università Politecnica delle Marche

Dr. Filippo Tassinari - Istituto Carlo Cattaneo, DSE Università di Bologna

Dr. Andrea Zoboli - Istituto Carlo Cattaneo

Si ringraziano per la preziosa collaborazione:

Per l'Università di Bologna il Prorettore Vicario Mirko Degli Esposti e il dott. Danilo Roberto Cinti, responsabile Ufficio Data Warehouse e rilevazioni ministeriali.

Per ER.GO., la Dott.ssa Maria Cristina Scarazzini

Per ACER e AMA l'Avv. Carmela Cappello e la Dott.ssa Cinzia Minarini

Per FIAIP, il Presidente Gianluca Giordani.

Per ASPPI, il Presidente Amedeo Pangrazi.

Per Confabitare, il Presidente Alberto Zanni.

Per A.I.P.I.- AssoProprietari, il Presidente Tommaso Veronesi.

Questa pubblicazione è l'esito dell'attività d'indagine affidata dal Comune di Bologna all'Istituto Carlo Cattaneo con atto P.G. N. 309572/2017 del 7 Settembre 2017

Isbn 9788894112634

Direzione e amministrazione: Fondazione di ricerca Istituto Carlo Cattaneo 40125 Bologna, Via Guido Reni, 5 - tel. +39 051239766

E-mail: istitutocattaneo@cattaneo.org

Sito: www.cattaneo.org 


\section{Sommario}

INDAGINE SUL MERCATO DEGLI ALLOGGI IN LOCAZIONE NEL COMUNE DI BOLOGNA.............. 1

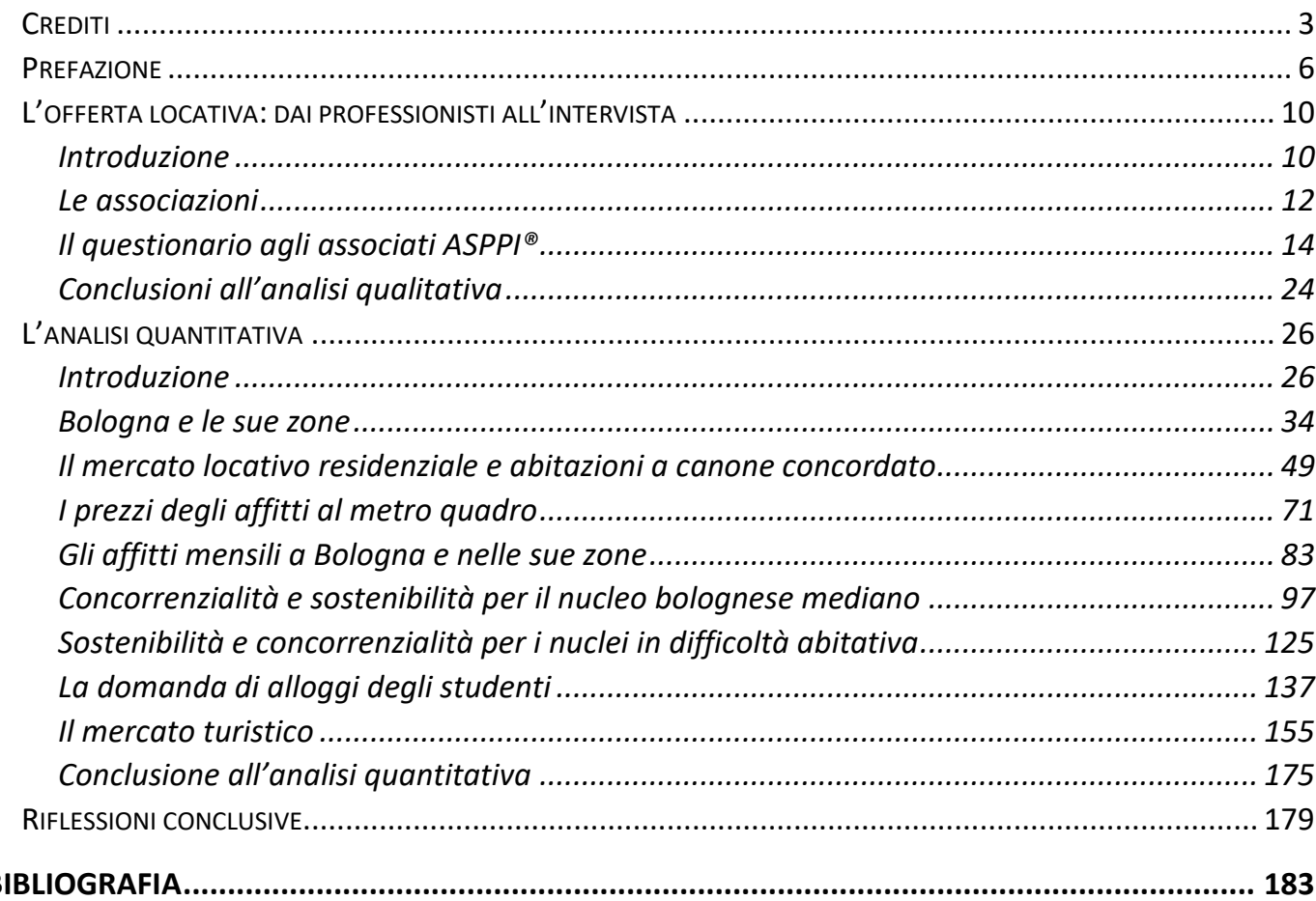




\section{Prefazione}

Nel settembre 2017 il Comune di Bologna ha commissionato la ricerca presentata in queste pagine all'Istituto Cattaneo, al fine di comprendere nel dettaglio le condizioni del mercato locativo bolognese. Bologna è infatti un caso particolare all'interno del panorama nazionale essendo investita da un flusso costante di studenti provenienti da fuori provincia che corrispondono a più di un quinto della popolazione cittadina.

La presenza di un flusso costante (e crescente negli ultimi anni) pari a 35'000 studenti circa ha effetti sia sulle dinamiche demografiche (circa il 25\% della popolazione residente nel Comune si rinnova ogni decennio) sia sui mercati dei beni e dei servizi. Sebbene l'Università sia una delle principali risorse cittadine, la presenza di un largo numero di studenti ha storicamente avuto effetti importanti sul mercato immobiliare, sia dell'acquisto che, in particolare, della locazione. I prezzi degli affitti a Bologna sono tra i più elevati del Paese e questo ha un forte impatto tanto sugli studenti (che sono la componente omogenea più consistente della domanda di locazioni) quanto sulle famiglie residenti che, spesso, hanno dovuto abbandonare il territorio comunale alla ricerca di prezzi meno elevati nell'hinterland. Se, da un lato, I'Università ha ricadute estremamente positive sulla città (fra cui l'ingente flusso di risorse economiche che gli studenti portano negli anni di permanenza), dall'altro non si può non annoverare tra le esternalità negative l'effetto sul mercato locativo per le famiglie.

Negli ultimi anni, la pressione sul mercato locativo è inoltre aumentata a causa di una rilevante crescita delle presenze turistiche in città. Il turismo, come verrà discusso, a fronte dell'indubbio beneficio che ha generato in termini economici per Bologna, si stima abbia sottratto in due anni circa 2'000 unità abitative a quel mercato locativo cittadino che era già sotto pressione. Nell'auspicio di una ulteriore crescita del turismo in città, tenendo in considerazione un'offerta alberghiera e di attività turistiche "classiche" fondamentalmente piatta, nuove opportunità fornite da servizi quali Airbnb $^{\mathrm{TM}}$ possono avere effetti positivi per l'economia cittadina e al contempo appesantire la condizione del mercato locativo. Si è voluto quindi provvedere ad una prima stima dell'effetto che il turismo ha su questo mercato e quale sia ad oggi il suo potenziale espansivo.

In questo contesto la ricerca è stata svolta su di un duplice canale. I due approcci, uno di tipo qualitativo e l'altro di tipo analitico quantitativo, sviluppati in contemporanea e separatamente, sono tuttavia giunti fondamentalmente agli stessi risultati. Da un lato si è provveduto ad una indagine di mercato prima prettamente qualitativa, col supporto degli operatori del settore, e poi tramite una prima indagine conoscitiva su circa 250 proprietari di immobili nel comune di Bologna. Dall'altro lato, la parte analitica è basata su una analisi del mercato locativo della città, utilizzando e rileggendo i dati forniti dell'Osservatorio del Mercato Immobiliare (OMI) dell'Agenzia delle Entrate, dal Comune di Bologna, da ISTAT, da Alma Mater Studiorum e, per il mercato turistico, da fonti indipendenti. 
L'analisi qualitativa, partendo dall'esperienza dell'Agenzia Metropolitana per l'Affitto (AMA), ripropone le informazioni raccolte in una serie di incontri con gli operatori del mercato immobiliare (intermediari, agenzia per l'affitto, associazioni di proprietari, operatori coinvolti nella gestione degli studentati).

Mentre le schede dei singoli incontri sono agli atti del Comune, in questo lavoro si riportano i principali argomenti trattati. Tali incontri, svolti coi rappresentanti e, a volte, in formato di focus con i soci, sono stati finalizzati a comprendere le caratteristiche generiche dell'utenza, le cause delle difficoltà di AMA e quali strumenti possano essere sviluppati e pensati per far sì che aumenti l'offerta, sia nel breve che nel lungo periodo. Gli incontri sono stati poi la base di partenza per la stesura di un questionario online atto a fornire una prima base conoscitiva del fenomeno in analisi. L'indagine è stata svolta nel mese di gennaio, tramite un questionario digitalizzato e telefonate, su tutto il parco dei soci ASPPI ${ }^{\circledR}$, associazione resasi molto disponibile nei confronti di tale ricerca. Poiché la normativa sulla privacy rende estremamente complesso raggiungere direttamente da parte dell'Istituto gli associati ASPPI ${ }^{\circledR}$, I'associazione ha invitato a tutti i soci un link al questionario al fine di raggiungere il massimo numero possibile di persone. II tasso di risposta è stato nell'ordine dell' $8 \%$ dei contattati, non dissimile dal tasso di risposta ottenuto da ASPPI ${ }^{\circledR}$ stessa due anni fa su di una indagine svolta in formato cartaceo sulle caratteristiche dei propri soci. Sebbene quindi l'indagine non possa essere considerata un campionamento corretto della popolazione dei proprietari immobiliari bolognesi, ha fornito interessanti spunti che non si distanziano nella propria struttura dai risultati nel frattempo emersi nell' analisi quantitativa.

Come detto, l'analisi quantitativa si basa invece su dati forniti direttamente dal Comune di Bologna e da altre fonti ufficiali. In tale analisi abbiamo incrociato le informazioni sui prezzi di mercato fornite dall'osservatorio OMI, lo stock di alloggi a canone concordato del Comune e le informazioni sulle condizioni economiche dei cittadini bolognesi (e di coloro che presentano domanda per gli alloggi ERP) al fine di comprendere la reale dimensione del mercato e se le difficoltà segnalate nel trovare alloggi in affitto siano da attribuirsi a specifiche condizioni di mercato, a particolari aree o all'insorgere di nuovi fenomeni. Tali informazioni sono integrate con quelle gentilmente fornite dall'Università in merito ai flussi di studenti nell'ultimo decennio, divisi per provincia di provenienza, oltre ai dati forniti da ER.GO. sulle richieste di supporto all'affitto, alle informazioni raccolte sulle residenze universitarie e ai dati disponibili nell'analisi svolta una decina di anni fa sulle condizioni degli studenti a Bologna. Infine, si sono utilizzate fonti indipendenti quali i dati raccolti da Tom Slee (professionista che si occupa da anni di analisi del mercato Airbnb ${ }^{\mathrm{TM}}$ ) al fine di poter quantificare l'effetto del turismo sul mercato locativo.

Come emergerà nelle prossime pagine, Bologna, negli ultimi 2 anni, presenta una offerta locativa piatta per non dire decrescente, a fronte di una domanda stabile o in crescita. Larga parte del mercato locativo è assorbita dagli studenti ai quali risulta strategicamente conveniente affittare, in particolare grazie ad alcuni vantaggi "de 
facto" e normativi. Per i proprietari, in particolare i piccoli proprietari particolarmente interessati a poter ritornare in tempi relativamente brevi in possesso del proprio immobile - spaventati da eventuali lunghi tempi di sfratto - risulta strategia dominante affittare agli studenti, a pari contratto, rispetto alle famiglie. In questa situazione, come verrà mostrato, il canone concordato rischia di non assolvere più alle funzioni per cui era originariamente stato concepito. Sebbene infatti il canone concordato risulti essere fortemente competitivo sul mercato cittadino in molte zone rispetto ai valori OMI, al punto da essere il traino dell'intero mercato in termini di prezzi, data la peculiarità dell'applicazione del canone concordato stesso anche a studenti, esso rischia di diventare al contempo un mezzo di esclusione delle famiglie.

La famiglia mediana bolognese appare infatti in difficoltà sul mercato locativo per le tipologie di alloggi che potrebbero essere di interesse per tali nuclei, ed in particolare appare generalmente più conveniente l'acquisto fuori dall'area comunale piuttosto che l'affitto in città. Le famiglie in difficoltà economica sono letteralmente escluse dalla possibilità di competere sul mercato per il livello degli affitti, dovendo quindi sperare negli alloggi di Edilizia Residenziale Pubblica (ERP) o dovendosi allontanare, spesso non di poco, dal comune stesso.

Il mercato dell'affitto turistico a breve ha potenziale di espansione economica non indifferente; alle attuali condizioni, infatti, l'offerta di alloggi di tipo Airbnb ${ }^{\text {TM }}$ potrebbe tranquillamente più che raddoppiare mantenendo un buon margine di profittabilità, ma sottraendo all'offerta locativa almeno altre 2'000 unità abitative. Questo, supponendo che il flusso sia stabile e arresti la propria crescita. In caso di ulteriore espansione agli attuali tassi, potenzialmente l'offerta (e di conseguenza la riduzione della stessa nel mercato locativo) può triplicare in poco tempo.

Come emergerà sia dall'indagine di mercato che dall'analisi quantitativa, l'offerta abitativa nel comune di Bologna appare sottodimensionata, o meglio, è tale da trovare equilibrio nel mercato ad un livello di prezzi particolarmente oneroso per le famiglie. In questo lavoro si farà "colloquialmente" riferimento ad eccesso di domanda e di mancanza di offerta in questi termini. Non si intende dire che vi sia domanda in sé non soddisfatta (come direbbe Adam Smith, la mano invisibile del mercato funziona benissimo), ma semplicemente che a queste condizioni l'equilibrio di mercato viene raggiunto a prezzi particolarmente elevati se rapportati ai redditi delle famiglie. Questo ha ripercussioni sulla qualità di vita delle famiglie, sulle loro capacità di spesa in altri beni e servizi e, soprattutto, spinge un consistente numero di famiglie a trovare alloggio fuori dai confini del Comune. Poiché il bene casa è uno di quei beni a particolare valenza sociale, la questione viene trattata nell'ottica di una famiglia mediana di Bologna e di coloro che sono in difficoltà economica al fine di valutare anche l'aspetto sociale di tali prezzi. Sempre in quest'ottica quindi si tengono in considerazione le ricadute sociali legate ad alti prezzi di affitto.

La leva fiscale appare come la strategia di più immediato effetto nel riallocare i contratti verso prezzi calmierati; tuttavia questa leva è in gran parte fuori dal controllo del Comune 
Nelle prossime pagine, oltre all'analisi, si proporranno alcune valutazioni di merito sullo specifico problema. In termini generali, tuttavia, si può anticipare come sia necessario pensare ad una strategia di sviluppo urbano, concordata tra i vari attori in causa, che vada ad aumentare l'offerta locativa o che riallochi la domanda nell'area metropolitana. 


\section{L'offerta locativa: dai professionisti all'intervista}

\section{Introduzione}

Questa prima parte della ricerca riporta le informazioni ottenute durante una serie di incontri individuali e di focus group con gli operatori (intermediari e associazioni di categoria) del settore immobiliare nel Comune di Bologna (e in alcuni casi anche nelle aree esterne al nucleo urbano centrale della città metropolitana) e i risultati di una indagine svolta con il supporto di ASPPI ${ }^{\circledR}$. Quest'ultima, sviluppata secondo le indicazioni ottenute negli incontri con i professionisti del settore, è stata concepita al fine di interrogare direttamente i proprietari di immobili nel comune di Bologna riguardo alla loro esperienza da conduttori e alle tipologie di incentivi e disincentivi (diretti o indiretti) che fanno sì che essi mantengano le proprie proprietà sul mercato dell'offerta (o che da esso si ritirino). Si vuole precisare come gli incontri con gli operatori di mercato siano riportati agli atti del Comune come schede. Le osservazioni svolte dagli operatori stessi e quindi l'inquadramento contestuale è brevemente riportato nella prima parte di questo capitolo.

L'indagine conoscitiva, che è stata sviluppata tramite interviste telefoniche e questionario online, ha ricevuto la risposta di circa 250 proprietari; sebbene non statisticamente rappresentativa della città, permette come prima indagine di far emergere alcune questioni che collimano con i risultati dell'analisi quantitativa. $\mathrm{Ne}$ emerge un quadro abbastanza chiaro, in cui la grandissima parte delle abitazioni eccedenti quella ad uso personale sono affittate (70\%). Se si escludono le case utilizzate per altri membri della famiglia, solo il $10 \%$ delle proprietà viene dichiarato sfitto. Fra gli alloggi affittati, larga quota (70\%) va a canone concordato, mentre il restante a prezzi di mercato. Come si evincerà anche nell'analisi quantitativa, la cedolare secca agevolata per i canoni concordati ha prodotto, negli ultimi anni, un importante trasferimento di contratti, prima a canone libero, verso questa tipologia contrattuale.

Professionisti e proprietari sottolineano comunque alcune difficoltà sul mercato, in particolare in merito all' affidabilità economica degli inquilini. Indubbiamente questo aspetto è centrale e ha risvolti sociali preoccupanti. In particolare, giovani coppie e immigrati sono i destinatari principali di queste preoccupazioni, sia per il timore di morosità e difficoltà economiche (l'attuale clima nel Paese non è certo favorevole, in particolare nei confronti degli stranieri) che per la paura di non riuscire più a rientrare in possesso dell'immobile. Nei confronti delle giovani coppie, laddove procreino, sarebbe più complesso eseguire uno sfratto e questo influisce indubbiamente sui comportamenti dei proprietari. Morosità e impossibilità di liberarsi dell'inquilino moroso sono nettamente le maggiori preoccupazioni che emergono, sia negli incontri con gli operatori di mercato, che dalle interviste ai proprietari. 
Sebbene le agevolazioni fiscali appaiano la leva principale per espandere ulteriormente l'offerta, risulta più complesso identificare uno spazio di espansione dell'offerta stessa, considerando che le abitazioni sembrano in larga misura già affittate. In queste condizioni, quindi, lo spazio di manovra sembrerebbe alquanto ridotto, se non nullo, in termini di politiche che favoriscano l'accesso ad abitazioni per le categorie più deboli. Gli studenti universitari, i quali scontano la preoccupazione dei conduttori nei confronti di eventuali danni, appaiono comunque essere preferiti rispetto a chi, all'occhio del conduttore (e spesso correttamente) sembri essere meno affidabile dal punto di vista economico. 


\section{Le associazioni}

Tra settembre e ottobre 2017, sono stati svolti una serie di incontri e focus group con gli operatori del settore immobiliare, al fine di cogliere criticità e punti di forza del mercato locativo bolognese. Gli attori con cui ci siamo interfacciati sono stati ACER e AMA, FIAIP, ASPPI, Confabitare, A.I.P.I. - Assoproprietari, ed ER.GO. ${ }^{1}$.

Sono state redatte delle schede riassuntive di questi incontri che sono agli atti depositati al Comune. In questa sede, dato il quadro omogeneo evidenziato trasversalmente da tutti gli operatori, si è ritenuto più opportuno presentare una sintesi complessiva, analizzando i temi che sono emersi in tutti gli incontri.

Tema iniziale di ogni discussione è stata l'Agenzia Metropolitana per l'Affitto (AMA)2: un'associazione senza fini di lucro nata con l'intento di agevolare l'incontro tra domanda e offerta di abitazioni, a vantaggio di potenziali inquilini con redditi troppo alti per accedere all'edilizia residenziale pubblica, ma troppo bassi per sostenere $i$ canoni di libero mercato. In tutti gli incontri è emerso come AMA sia stata un'esperienza negativa, che non ha portato ai risultati sperati. Tra le ragioni più comuni è stata riscontrata l'inefficacia della strategia pubblicitaria adottata per diffondere la conoscenza del servizio e comunicarne le modalità di accesso, oltre ad altre ragioni legate alla struttura stessa di AMA e del mercato in cui questa si inseriva. Nello specifico, l'impossibilità per il proprietario di scegliere personalmente I'inquilino, una generale sfiducia verso l'istituzione pubblica (che nel caso di AMA si pone come intermediario tra locatore e locatario) ed infine l'elevata competitività del mercato di riferimento. In questo senso l'elevata domanda d'affitto permette al locatore di affittare in tempi brevi e con estrema facilità ${ }^{3}$.

Quest'ultima ragione merita un ulteriore sviluppo, in quanto centrale per cogliere l'andamento del mercato locativo cittadino. Tutte le associazioni di categoria hanno sottolineato la presenza di un mercato fortemente dinamico, con un'elevata domanda a fronte di una scarsa offerta. In quest'ottica i tempi di sottoscrizione del contratto sono molto brevi e il proprietario può sempre affittare con facilità scegliendo fra un parco di conduttori. Questa possibilità di scelta dell'inquilino spesso si traduce in una maggiore difficoltà per le famiglie straniere nel trovare una soluzione abitativa in quanto, a detta degli intermediari, i proprietari preferiscono affittare ad altre tipologie familiari.

Un terzo tema emerso trasversalmente in tutti gli incontri è l'efficacia dei contratti a canone concordato. Si tratta di una forma contrattuale molto apprezzata dai

\footnotetext{
${ }^{1}$ L'incontro con ER.GO., a differenza degli altri, è stato specifico per il mercato immobiliare locativo studentesco.

${ }^{2}$ http://www.ama.bo.it/1/1/il-progetto

${ }^{3}$ Affidarsi ad AMA comporterebbe un allungamento delle tempistiche per la stipula del contratto e la percezione di un canone uguale o inferiore.
} 
proprietari, grazie principalmente alle riduzioni fiscali destinate loro, che permette alle famiglie di sostenere canoni d'affitto inferiori rispetto a quelli del libero mercato. In tutte gli incontri questa risulta essere la forma contrattuale preferita dagli associati e viene adottata nella maggior parte delle stipule. Tale tipologia contrattuale non viene però uniformemente adottata su tutto il territorio cittadino: in centro, essendo i prezzi vantaggiosi in quest'area, i contratti a canone concordato sono largamente utilizzati; diversamente nelle zone periferiche, a causa della bassa profittabilità, $\mathbf{i}$ contratti a libero mercato continuano ad essere maggiormente prediletti.

Un ultimo tema che è emerso in tutti gli incontri è quello dell'affitto a breve termine. Si sta assistendo ad un trend che va sempre di più in questa direzione, favorendo quindi il turismo a discapito delle famiglie. Sempre più abitazioni, infatti, vengono sottratte dal mercato locativo residenziale per essere destinate a piattaforme dedite all'affitto turistico breve che assicura maggiori margini di guadagno e minori rischi. In particolare, questo fenomeno si concentra nelle zone del centro storico, della Fiera e delle strutture ospedaliere.

Complessivamente, il quadro che emerge da tutti gli incontri suggerisce come l'introduzione degli accordi per la definizione dei contratti a canone concordato sia una soluzione efficace, ma non sufficiente, per sostenere le famiglie in un mercato in cui l'eccesso di domanda determina uno squilibrio di potere a vantaggio dei proprietari. In quest'ottica alcune delle associazioni intervistate vedono nel social housing un possibile strategia efficace per ridurre la distanza tra domanda e offerta nel mercato locativo cittadino. 


\section{|l questionario agli associati ASPPI ${ }^{\circledR}$}

\section{Obiettivi e metodologia}

Obiettivo di questa sezione dello studio è quello di cogliere atteggiamenti e orientamenti dei proprietari relativamente alla situazione del mercato locativo residenziale nel comune di Bologna. L'analisi evidenzia un risultato per certi versi atteso, in buona parte già emerso negli incontri preparatori con gli operatori di mercato, ossia la presenza di un problema abitativo dovuto ad un eccesso di domanda rispetto all'offerta.

Per cercare di approfondire le ragioni di una disponibilità di affitti tale da comportare prezzi sul mercato particolarmente elevati, occorre indagare a fondo le ragioni dei proprietari, di chi cioè determina l'offerta. A questo scopo è stato possibile sottoporre ad alcuni di essi un questionario strutturato, appositamente predisposto in seguito agli incontri organizzati con le associazioni di proprietari e inquilini.

Il questionario, realizzato attraverso la piattaforma "Google Form"4, è allegato in appendice a questo report. Non disponendo delle informazioni necessarie per prevedere un campionamento che coprisse l'intera popolazione di proprietari immobiliari bolognesi, ci si è giovati della collaborazione di una delle principali associazioni di categoria, $\mathrm{ASPPI}^{\circledR}$, che ci ha consentito di sottoporre tale questionario ad una parte dei suoi associati ${ }^{5}$. La somministrazione è avvenuta attraverso l'inoltro alla mailing-list, effettuata in data 30 gennaio 2018, con un secondo invio (recall) ad una settimana di distanza. II periodo di somministrazione va dal 30 gennaio al 14 febbraio 2018. Parallelamente, il medesimo questionario è stato sottoposto a 30 associati, dei quali ci era stato fornito un contatto telefonico personale: 22 di essi hanno portato a termine l'intervista.

Combinando le due diverse metodologie di somministrazione, sono state ottenute 279 risposte. Tale platea di rispondenti, dettagliatamente descritta nella sua composizione, non garantisce una piena rappresentatività della popolazione di riferimento, ciò nonostante offre numerosi spunti di interesse e risultati meritevoli di attenzione.

Nello specifico vengono sostanzialmente confermate le informazioni ottenute durante i colloqui con gli operatori di mercato, ma soprattutto emerge la scarsa possibilità di espandere l'offerta di mercato. II 70\% degli immobili è già destinato all'affitto e del restante $30 \%$ solo un $10 \%$ è fuori mercato realmente perché il $90 \%$ è già allocato a parenti $\mathrm{o}$, comunque, mantenuto fuori mercato per intenzioni legate ad esigenze familiari.

\footnotetext{
${ }^{4}$ https://www.google.it/int//it/forms/about/

${ }^{5}$ Secondo quanto dichiarato da ASPPI il proprio database contiene circa 3000 indirizzi email.
} 


\section{Caratteristiche socio-demografiche degli intervistati}

Il campione si compone di 279 rispondenti, 22 dei quali intervistati telefonicamente, mentre i restanti 257 hanno autonomamente compilato il questionario online. La Figura 1 mostra una ripartizione di genere pressoché uniforme: 142 uomini e 137 donne.

Figura 1. Genere rispondenti.

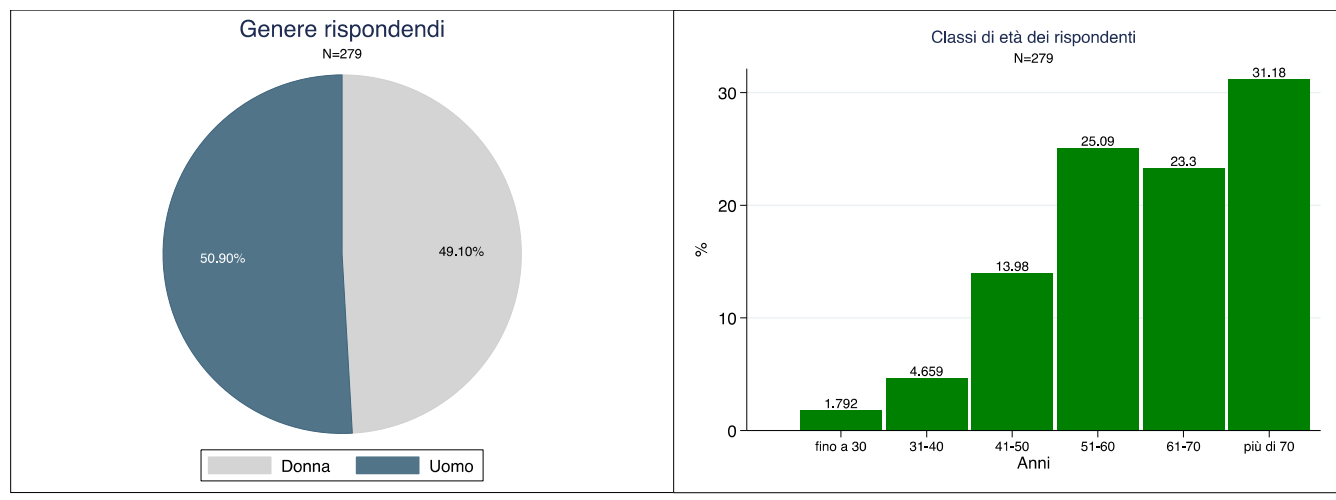

Figura 2. Frequenza percentuale rispondenti per classe di età.

L'età media è di 62.4 anni, con una deviazione standard ${ }^{6}$ di 14.3 anni; il rispondente più giovane ha 23 anni, quello più anziano 100. Il dato è confermato dalla Figura 2 che rappresenta la ripartizione degli intervistati per classe d'età. La classe modale, che registra il 31.18\% dei rispondenti, è quella degli over 70 . Complessivamente il $79.57 \%$ ha un'età superiore ai cinquant' anni.

Data l'età media piuttosto avanzata degli intervistati, non stupisce notare la cospicua presenza di pensionati $(44,7 \%$ degli intervistati), categoria seconda in termini di frequenza soltanto agli occupati (49,5\% del totale); marginale è invece la presenza di casalinghe $(4,7 \%)$ e ancor meno sono i disoccupati (Figura 3). Per ciò che concerne quanti si dichiarano occupati, il $75 \%$ lavora nel settore privato, mentre il restante $25 \%$ nel pubblico.

${ }^{6}$ Vedesi glossario 
Figura 3. Condizione occupazionale rispondenti.

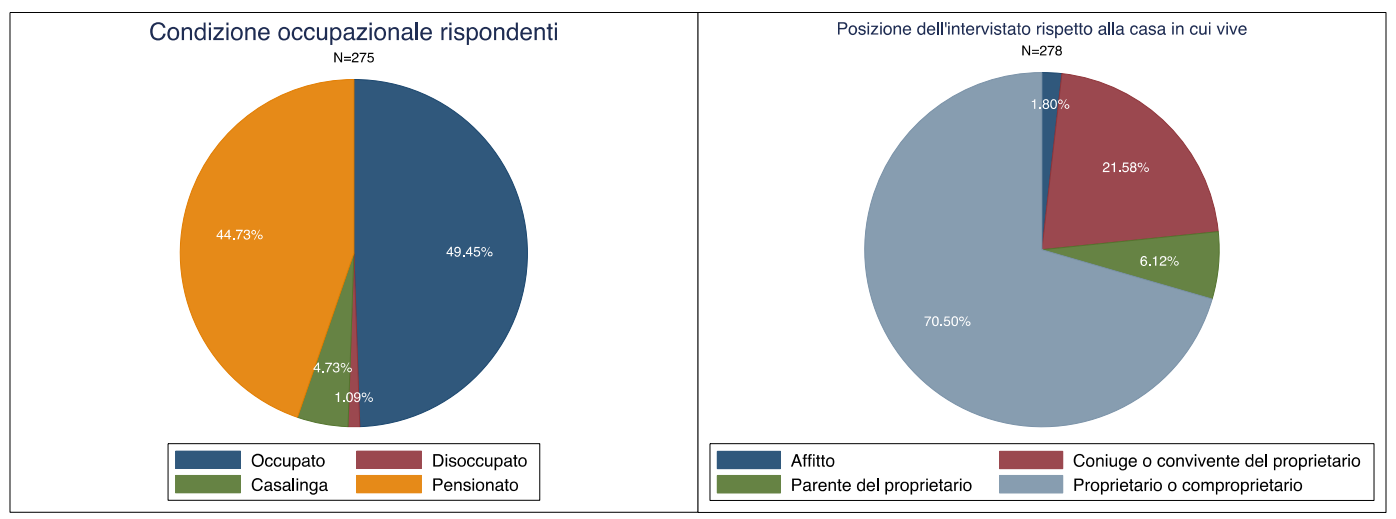

Figura 4. Posizione dell'intervistato rispetto all'abitazione in cui vive.

La Figura 4, infine, è relativa al titolo di godimento dell'abitazione di residenza: la maggior parte dei rispondenti è proprietario o comproprietario (70.50\%), quasi tre persone su dieci risultano coniugi o parenti del proprietario, mentre esiste anche una piccola percentuale $(1.8 \%)$ di associati $\mathrm{ASPPI}^{\circledR}$ che vive in affitto

\section{Le abitazioni possedute nel Comune di Bologna}

La Figura 5 evidenzia la distribuzione degli intervistati rispetto al numero di abitazioni possedute oltre alla residenza principale: uno su cinque dichiara di non essere in possesso di altre abitazioni; poco più di un terzo è intestatario di un solo stabile; chi possiede due o tre abitazioni - rispettivamente $20,4 \%$ e $12,9 \%$; infine, coloro per i quali si può ipotizzare una gestione imprenditoriale degli stabili - da quattro in su costituiscono il restante $12,5 \%$.

Figura 5. Numero di abitazioni possedute nel Comune di Bologna oltre all'abitazione principale.

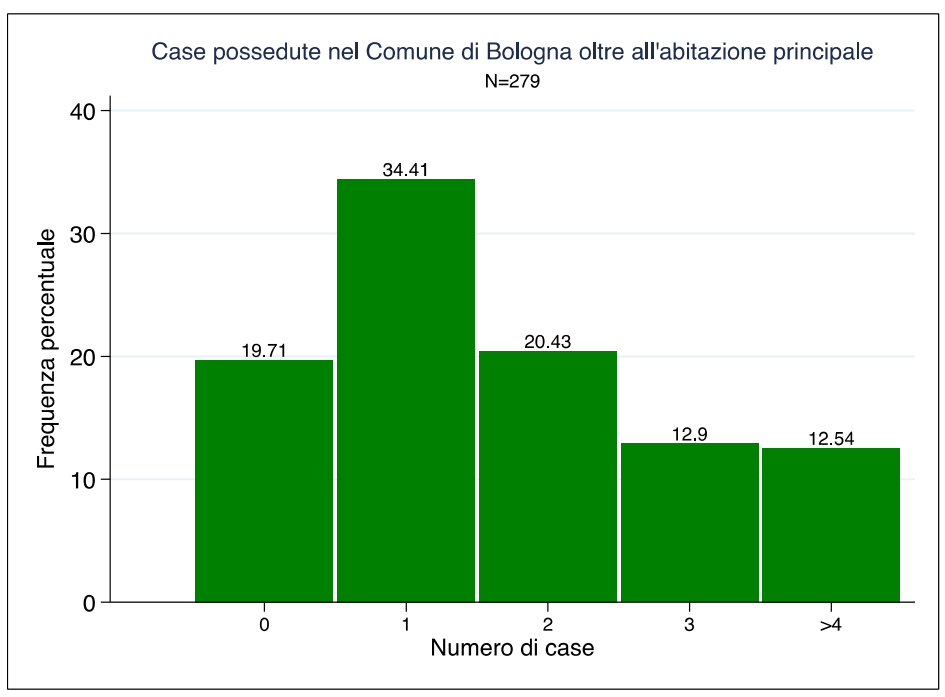


Tra gli intervistati, 200 (pari al 72\% del campione) dichiarano di possedere case destinate all'affitto nel comune di Bologna, concorrendo a formare un totale di 415 abitazioni (dunque una media di 2,11 a testa, con un massimo di dieci alloggi riconducibili al medesimo proprietario). A questo proposito, la Figura 6 evidenzia che tra i 200 locatori, il 48,5\% sono proprietari di una sola abitazione, il 38,5\% titolari di due o tre immobili oltre alla residenza principale, ed il restante $13 \%$ è composto da intestatari di patrimoni immobiliari più consistenti (da 4 case in su).

Figura 6 Frequenza percentuale per numero di abitazioni possedute e destinate all'affitto nel Comune di Bologna.

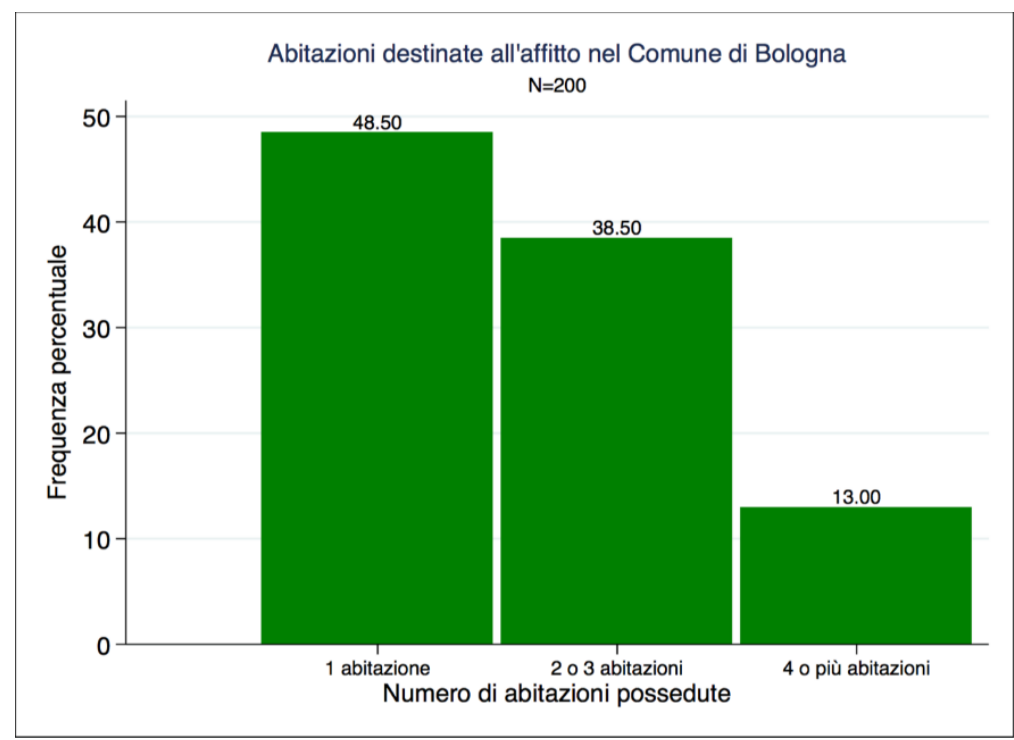

La forma contrattuale cui si affidano prevalentemente i proprietari di abitazioni destinate all'affitto si evince osservando la Figura 7 e la Figura 8. Vi è un ampio ricorso a contratti a canone concordato (applicati al 76,1\% del parco abitazioni), mentre i contratti in regime di libero mercato costituiscono una scelta minoritaria $(17,4 \%$ del parco abitativo destinato all'affitto); si segnala infine una quota minoritaria di abitazioni attualmente sfitte, in attesa di locazione, che si attesta al 6,5\% del totale ${ }^{7}$.

La preferenza per i contratti a canone concordato diminuisce tra i proprietari di quattro o più abitazioni, per i quali aumenta l'incidenza degli accordi sottoscritti a canone libero. Da ultimo, si segnala che la percentuale di abitazioni momentaneamente non occupate, per quanto residuale, cresca tra gli intestatari di più abitazioni (rispettivamente 7,5\% e 7,7\%), raggiungendo valori doppi rispetto a quanto registrato tra $\mathrm{i}$ proprietari di un singolo alloggio $\mathrm{i}$ quali, probabilmente, possono permettersi di tenere l'abitazione sfitta in attesa di un contratto pienamente

\footnotetext{
7 Si noti che il totale delle abitazioni considerate è di 397, e non 415 come precedentemente emerso rispetto al numero di abitazioni in affitto: sono state infatti escluse le risposte non coerenti da parte di 7 rispondenti.
} 
soddisfacente per un periodo di tempo minore rispetto a chi detiene un patrimonio immobiliare superiore.

Figura 7. Tipologia di locazione per le abitazioni destinate all'affitto.

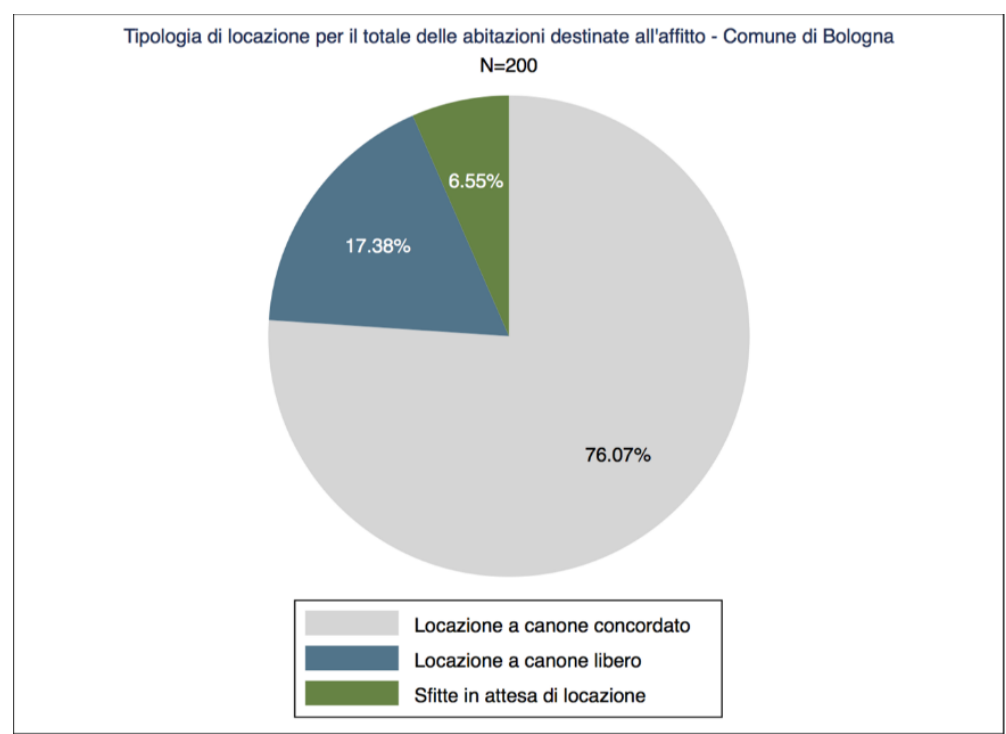

Figura 8. Tipologia di locazione suddivisa per numero di case possedute e destinate alla locazione.

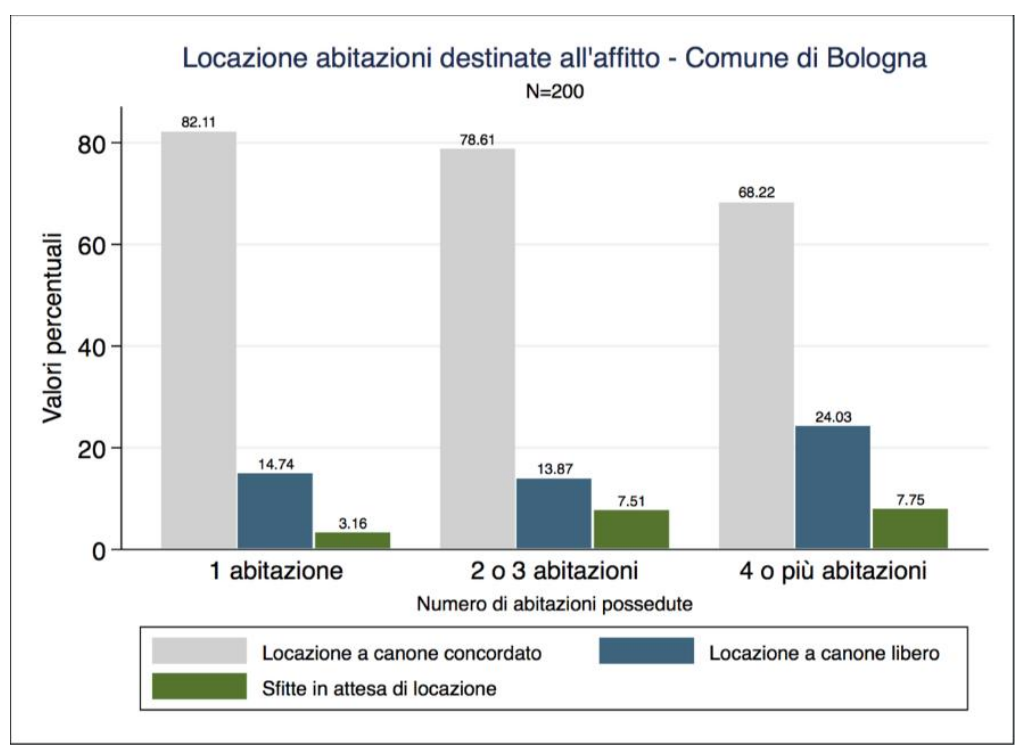

Esaminati i proprietari di abitazioni in affitto e le forme contrattuali applicate, va rimarcata una componente di intervistati che dichiara di possedere case esplicitamente non destinate all'affitto: si tratta, nel dettaglio, di 52 rispondenti, tra i quali sono suddivise 63 abitazioni. Come confermato dalla Figura 9, la ragione prevalente alla base della scelta di non accedere al mercato locativo è da individuare 
nell'utilizzo delle suddette case da parte di familiari (l'88\% degli interessati) e solo secondariamente nell'influenza di esperienze negative con i conduttori precedenti (8\%) o negli eccessivi costi di ristrutturazione (4\%).

Figura 9. Ragioni per cui il proprietario decide di non destinare all'affitto alcune delle abitazioni possedute nel comune di Bologna.

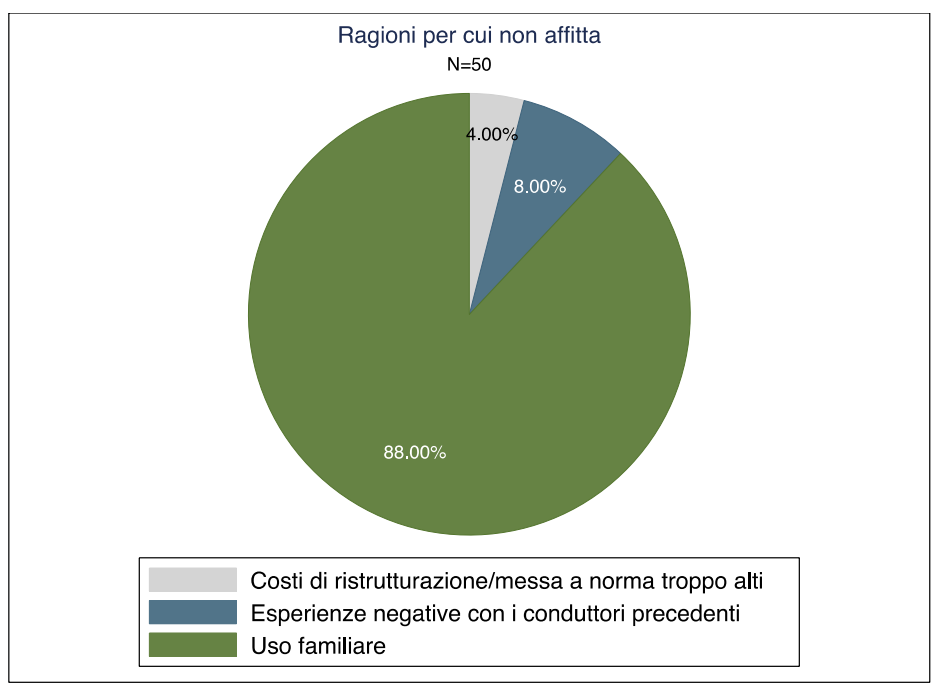

A conclusione di questa ricognizione del patrimonio immobiliare degli intervistati, e del relativo impiego, l'ipotesi che le abitazioni sfitte, sulla cui entità molto si discute anche per la complessità di rilevarle esattamente, possano avere un'incidenza modesta sul totale del mercato della locazione a Bologna, appare meritevole di una verifica. Se il cosiddetto sfitto appare infatti una soluzione di breve periodo di interesse per il policy maker, questa prima indagine farebbe pensare ad un patrimonio immobiliare sfitto (e non finalizzato alla vendita) non particolarmente consistente. Poiché però si tratta di una preliminare indagine, questo punto richiede assolutamente più profonda investigazione.

\section{Le precedenti esperienze di locazione}

Una volta definite le tipologie di proprietà, l'entità del parco case in affitto, le forme contrattuali praticate e le eventuali ragioni per il mancato accesso al mercato, si è provveduto a raccogliere informazioni rispetto alle esperienze di locazione, passate $o$ in corso, evidenziando eventuali elementi utili a favorire un accordo di affitto e i fattori di preoccupazione negativi per lo stesso. Si è inoltre indagata la disponibilità ad ospitare particolari gruppi di inquilini. 
Tabella 1. "Da 0 a 5, quanto ritiene importanti i seguenti fattori per una locazione?". Punteggi medi, deviazione standard, moda e \% di risposte " 5 , massimamente importante". N rispondenti $\mathbf{2 7 9}$.

Da 0 a 5, quanto ritiene importanti i seguenti fattori per una locazione?

\begin{tabular}{|l|r|r|r|r|}
\hline & Media & $\begin{array}{c}\text { Deviazione } \\
\text { standard }\end{array}$ & Moda & $\begin{array}{c}\text { \% di } \\
\text { giudizio } \\
\text { massimo }\end{array}$ \\
\hline $\begin{array}{l}\text { Agevolazioni fiscali sul reddito } \\
\text { (es. "Cedolare secca") }\end{array}$ & 4.57 & 0.98 & 5 & 77.82 \\
\hline $\begin{array}{l}\text { Agevolazioni sulle imposte locali } \\
\text { (es. riduzione dell'IMU) }\end{array}$ & 4.56 & 0.98 & 5 & 77.82 \\
\hline Possibilità di selezione dell'inquilino & 4.04 & 1.20 & 5 & 43.58 \\
\hline Affidabilità economica dell'inquilino & 4.70 & 0.71 & 5 & 79.38 \\
\hline Minor durata del contratto di locazione & 2.81 & 1.51 & 3 & 17.51 \\
\hline
\end{tabular}

La Tabella 1 sintetizza le risposte relative al livello di importanza attribuito ad una serie di fattori tesi a favorire la locazione di un'abitazione, siano esse di natura fiscale, contrattuale o riferite all'inquilino. Emerge in maniera significativa l'esigenza di tutelarsi economicamente da rischi di morosità, ragion per cui l'affidabilità dell'inquilino è giudicata massimamente importante da 4 proprietari su 5 (media=4,7). Altrettanto significative sono le agevolazioni fiscali, come la cedolare secca sugli affitti ( $m e d i a=4,57$ ) e riduzioni sulle imposte locali, come I'IMU (media=4,56). Un peso inferiore, ma comunque importante, viene affidato alla possibilità di selezione dell'inquilino (media=4,04), da leggersi probabilmente non tanto dal punto di vista della solidità economica, quanto piuttosto come volontà di individuare inquilini congeniali alle proprie aspettative e consuetudini di affitto. Infine, non pare particolarmente interessante, per i proprietari intervistati, la prospettiva di contratti di minore durata, grazie ai quali poter rientrare in pieno possesso dello stabile in tempi inferiori a quanto previsto finora (media $=2,81$ ).

Tabella 2. "Da 0 a 5, quanto ritiene preoccupanti i seguenti elementi per una locazione?". Punteggi medi, deviazione standard, moda e \% di risposte " 5 , massimamente preoccupante". N rispondenti $\mathbf{2 7 9}$.

\begin{tabular}{|l|r|r|r|r|}
\hline \multicolumn{2}{|c|}{ Da 0 a 5, quanto ritiene preoccupanti i seguenti elementi per una locazione? } \\
\hline & Media & $\begin{array}{c}\text { Deviazione } \\
\text { standard }\end{array}$ & Moda & $\begin{array}{c}\text { \% giudizio } \\
\text { massimo }\end{array}$ \\
\hline Morosità & 4.80 & 0.68 & 5 & 90.27 \\
\hline Inquilino non italiano & 2.90 & 1.55 & 4 & 16.34 \\
\hline Danni alla casa & 4.42 & 0.92 & 5 & 61.87 \\
\hline Durata del contratto & 2.77 & 1.49 & 3 & 15.56 \\
\hline Tempistiche di sfratto & 4.47 & 1.15 & 5 & 77.04 \\
\hline
\end{tabular}

La Tabella 2 è riferita alla domanda tesa ad esplorare le principali preoccupazioni dei locatori nel corso di un'esperienza d'affitto. La morosità degli inquilini è la prospettiva 
più sgradita, confermando indirettamente l'importanza attribuita all'affidabilità economica della controparte nella domanda precedente: la media è di 4,8 con oltre il $90 \%$ dei rispondenti che ha dato il massimo punteggio. L'incapacità dell'inquilino di far fronte agli impegni economici può essere una possibile causa di sfratto: procedura, quest'ultima, che preoccupa i rispondenti (media=4,47) più dei rischi di danneggiamenti dell'abitazione che portino a costose manutenzioni e a svalutazione dell'immobile (media 4,42, seppur con un numero di risposte "estremamente preoccupante" inferiore ai fattori citati in precedenza). Più difficile interpretare l'orientamento del campione rispetto alle prospettive di convivenza con un inquilino straniero, in quanto la risposta potrebbe incappare in un effetto di desiderabilità sociale tale per cui atteggiamenti estremi o ritenuti contrari al sentire prevalente verrebbero celati, dichiarando posizioni più moderate. La media si attesta a 2,90, ma si registra una notevole variabilità intorno a tale tendenza centrale; la modalità prevalente risulta corrispondere all'opzione di risposta 4 ed il numero di giudizi esprimenti preoccupazione (dunque da 3 a 5) risulta alquanto superiore a quelli che esprimono posizioni più tolleranti. Potremmo concludere che si percepisce una certa diffidenza di fondo la quale, pur non sfociando in atteggiamenti apertamente ostili, merita comunque attenzione. Infine, non è particolarmente temuta la durata del contratto (media $=2,77$ ), a conferma di quanto detto in precedenza relativamente alla poca importanza attribuita a forme contrattuali più brevi.

Tabella 3. "Su una scala da 0 a 5 in cui 5 indica la massima disponibilità, in che misura è disponibile ad affittare ai seguenti soggetti?". Punteggio medio, deviazione standard, \% di risposte che rivelano disponibilità, \% di risposte "massima disponibilità". N rispondenti=279.

\begin{tabular}{|l|r|r|r|r|}
\hline \multicolumn{1}{|c|}{ Disponibilità ad affittare a diversi soggetti (valori da 0 a 5) } \\
\hline
\end{tabular}

La Tabella 3 e la Figura 10 riguardano l'ultima domanda della sezione del questionario relativa alle esperienze di locazione. Data la vastità e la diversità della domanda di alloggi a Bologna, il proprietario di un immobile è nelle condizioni di avere a che fare con differenti tipologie di inquilino, per composizione del nucleo familiare, affidabilità 
economica e profilo sociale. In questo senso bisogna ricordare la composizione della platea di intervistati, di età media elevata e costituito in buona parte da pensionati.

Figura 10. Disponibilità ad affittare a diverse tipologie di inquilino: \% risposte tendenziale indisponibilità ( $d a \mathrm{O}$ a 2) e tendenziale disponibilità (da 3 a 5).

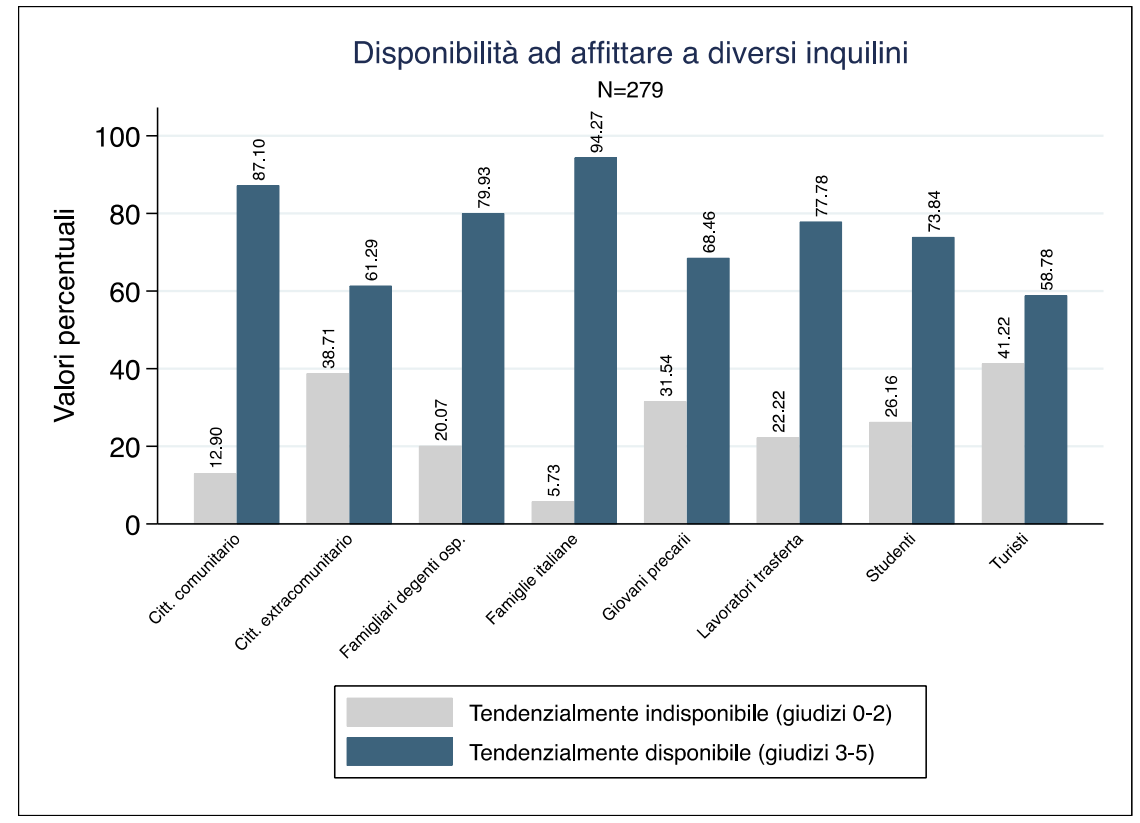

La maggiore disponibilità all'affitto è dimostrata nei confronti delle "famiglie italiane" (media=4,47): si tratta di un'immagine, d'altronde, che evoca stabilità e sicurezza, sia in termini di durata della permanenza che in termini di stili di vita e garanzie economiche. Per contrasto con le caratteristiche appena enunciate, emergono i profili dei conduttori meno graditi: in primis i turisti (media $=2,55$ ) interessati a soggiorni brevi e in seconda battuta i cittadini stranieri, anche in ragione della diffidenza evidenziata in precedenza. All'interno di questo intervallo si collocano altri profili, tra cui studenti, famigliari di degenti e lavoratori in trasferta (dei quali probabilmente non si apprezzano le prospettive di soggiorno a breve termine) e i giovani precari, che evidentemente non forniscono adeguate garanzie economiche. In merito all'atteggiamento nei confronti degli stranieri, influisce fortemente nella percezione dell'intervistato il termine "extracomunitari" rispetto al termine "comunitari". Tale differenza vale oltre un punto in più di media in termini di disponibilità all'accoglienza.

\section{Possibili incentivi alla locazione}

Agli iscritti $\mathrm{ASPPI}^{\circledR}$ intervistati si è infine richiesto un giudizio relativamente ad alcune possibili misure volte a incentivare la locazione. Rispetto a cinque diversi provvedimenti ipotizzati, ogni rispondente era chiamato a selezionare il primo, il secondo ed il terzo per importanza. La Tabella 4 riporta i risultati emersi. 
Tabella 4. Possibili incentivi alla locazione. Ogni intervistato era chiamato ad indicare il primo, il secondo ed il terzo per importanza. Totale menzioni e \% menzioni come primo, secondo o terzo elemento più importante. $\mathrm{N}$ rispondenti=279.

\begin{tabular}{|l|r|r|r|r|}
\hline \multicolumn{1}{|c|}{ Incentivo } & \multicolumn{1}{|c|}{$\begin{array}{c}\text { Totale } \\
\text { Menzioni }\end{array}$} & $\begin{array}{c}\text { \% } \\
\text { Primo }\end{array}$ & $\begin{array}{c}\text { \% } \\
\text { Secondo }\end{array}$ & $\begin{array}{c}\% \\
\text { Terzo }\end{array}$ \\
\hline Sensibile riduzione IMU & 200 & 38.32 & 23.90 & 11.03 \\
\hline $\begin{array}{l}\text { Fondo di garanzia gratuito contro il rischio di } \\
\text { morosità, a copertura di massimo 12 mensilità } \\
\text { del canone non corrisposto }\end{array}$ & 235 & 43.80 & 30.88 & 11.40 \\
\hline $\begin{array}{l}\text { Tutela legale gratuita in caso di morosità del } \\
\text { conduttore }\end{array}$ & 185 & 8.03 & 20.22 & 39.71 \\
\hline $\begin{array}{l}\text { Polizza assicurativa gratuita contro eventuali } \\
\text { danneggiamenti all'immobile prodotti dal } \\
\text { conduttore }\end{array}$ & 118 & 4.01 & 16.18 & 23.16 \\
\hline $\begin{array}{l}\text { Alla stipula del contratto, possibilità di incassare } \\
\text { anticipatamente 6 mensilità del canone di } \\
\text { locazione in un'unica soluzione, oltre la cauzione }\end{array}$ & 71 & 5.47 & 8.82 & 11.76 \\
\hline Nessuna delle misure indicate & & & & \\
\hline
\end{tabular}

L'incentivo più menzionato nel complesso, e più volte indicato come maggiormente significativo, è la costituzione di un fondo di garanzia contro il rischio di morosità, che copra fino a 12 mensilità di canone non corrisposto (235 menzioni). Segue in termini di gradimento la prospettiva di una sensibile riduzione dell'IMU a favore dei proprietari (200 menzioni). Ricorrenti, ma quasi mai individuati come intervento più importante, sono l'offerta di una tutela legale gratuita in caso di morosità del conduttore (185 menzioni) e la possibilità di stipulare un'assicurazione contro i danneggiamenti all'immobile (118 menzioni). Meno interessante risulta infine la possibilità di incassare anticipatamente 6 mensilità del canone di locazione, oltre alla cauzione (71 menzioni). In altri termini, possiamo osservare in questi risultati un riflesso delle esigenze e delle preoccupazioni evidenziate in precedenza: se la morosità dell'inquilino era la prospettiva più temuta, non stupisce che il primo e il terzo provvedimento ritenuti più importanti siano quelli mirati a tutelare dai rischi il proprietario. La riduzione delle aliquote IMU è invece facilmente riconducibile all'importanza attribuita alle agevolazioni fiscali, a partire dalla cedolare secca. 


\section{Conclusioni all'analisi qualitativa}

Stando agli associati ASPPI ${ }^{\circledR}$ intervistati e ai colloqui svolti con gli operatori e le associazioni di categoria, il mercato cittadino appare saturo con la domanda che esaurisce l'offerta. Non vi sono infatti spazi di notevole espansione del mercato stesso, data la scarsissima disponibilità di unità abitative sfitte. Nello specifico la forte domanda permette di affittare in tempi brevi e le agevolazioni fiscali legate alle forme di contratto a canone concordato, associate alla scarsa distanza tra i prezzi calmierati e quelli di mercato, rendono questa forma contrattuale molto interessante.

All'interno di questo contesto abbastanza roseo per i locatori, emergono comunque alcune difficoltà legate alla difficile gestione dell'inquilino moroso. Queste difficoltà, sebbene circoscritte ad una frazione ridotta dei locatori consultati, hanno una forte influenza sull'opinione complessiva degli intervistati, rendendo poco graditi quegli inquilini che sono universalmente più deboli dal punto di vista economico e sociale: giovani coppie, lavoratori precari e immigrati (ovvero coloro che maggiormente richiedono alloggi in affitto, non essendo nella posizione di ottenere un mutuo).

In questo contesto la leva fiscale (su cui già il Governo è intervenuto con la cedolare secca al $10 \%$ per i canoni concordati) appare il primo strumento di interesse dei locatori, seguito a breve distanza da tutele del patrimonio in caso di morosità dell'inquilino.

Va sottolineato come ampia parte del patrimonio immobiliare appartenga a soggetti in età avanzata e quindi potenzialmente non interessati all'intraprendere attività quali I'affitto a breve per scopo turistico; tuttavia non è da escludere che nei prossimi anni vi sia un consistente ricambio generazionale nella proprietà stessa e che quindi, anche attraverso attività di intermediazione, questo fenomeno si estenda in maniera considerevole.

La predisposizione all'affitto verso le famiglie italiane tende a scontrarsi con la poca predisposizione ad affittare a giovani coppie. Se la famiglia italiana è percepita come affidabile, i giovani no. La chiave di volta della questione risiede nella scarsa affidabilità economica (o nella percezione di scarsa affidabilità economica). Questa investe in maniera ancora maggiore i lavoratori a termine e i migranti. Sui migranti, infine, si deve sottolineare come vi sia un atteggiamento di diffidenza che sfocia spesso in posizioni di rifiuto. 


\section{L'analisi quantitativa}

\section{Introduzione}

L'analisi quantitativa del mercato locativo bolognese è stata svolta al fine di dare una prima stima del patrimonio immobiliare attualmente a mercato, con un particolare riguardo nei confronti di come questo si possa intrecciare con le differenti domande di spazi abitativi. Nel comune di Bologna, a fronte di un patrimonio immobiliare in larga parte costituito da prime case di proprietà, esiste un consistente mercato della locazione (in gran parte assorbito dai contratti di canone concordato) che deve soddisfare la domanda di circa 35'000 studenti fuori sede e delle famiglie. Questa duplice domanda oggi compete anche con l'emergente fenomeno dell'affitto turistico a breve termine. In particolare, negli ultimi 2 anni circa 2'000 unità abitative complete sono state destinate a questa forma di business.

Sebbene l'analisi sia una prima valutazione e pertanto non esaustiva del problema, tenendo in considerazione che la casa - intesa come luogo di vita e familiarità, rifugio e centro da cui parte la mobilità- non riguarda il solo mercato locativo, ma quello in generale dell'assetto strategico cittadino (che va dalla pianificazione urbana ai servizi di mobilità, passando per l'educazione, il welfare, il turismo, etc.), si ritiene che questo lavoro chiarisca la centralità del problema fornendo una prima informazione da cui partire.

Soluzioni semplici a quella che è una chiara carenza di unità abitative in locazione all'interno dell'area comunale non ve ne sono. Come già detto, s'intende carenza di alloggi il fatto che l'equilibrio di mercato venga raggiunto a prezzi particolarmente gravosi per la famiglia mediana bolognese e assolutamente non avvicinabili dai nuclei in difficoltà. Al fine di porre rimedio alla situazione, una profonda riflessione sulla struttura cittadina dovrà essere intrapresa assieme a futuri studi. Alle attuali condizioni infatti, al fine di rendere disponibili sul mercato unità abitative adatte alle esigenze delle famiglie (affitti più bassi, de facto) le soluzioni sono particolarmente complesse. Da un lato si potrebbe espandere l'offerta immobiliare (a locazione), ${ }^{8}$ dall'altro si potrebbe pensare di riallocare parte della domanda all'interno di uno spazio più ampio, quello della città metropolitana. ${ }^{9}$ Entrambe le soluzioni (o un mix delle stesse) infatti partono dall'ovvio presupposto che sia il turismo che l'Università

\footnotetext{
${ }^{8}$ All'interno del contesto normativo emiliano romagnolo e della nuova legge urbanistica regionale entrata in vigore il primo Gennaio 2018, si tende ad escludere la possibilità di sopperire a tali esigenze tramite "nuove costruzioni". L'aumento dell'offerta sarebbe quindi da sviluppare attraverso il recupero dello sfitto e la riqualificazione delle aree abbandonate

${ }^{9}$ La dislocazione della domanda è altresì una questione particolarmente delicata, considerando che la scelta abitativa non dipende generalmente solo dai prezzi e dalla qualità delle abitazioni ma anche da tutti quei servizi, di mercato e non, che rendono di interesse un territorio.
} 
sono patrimonio chiave della città. In questo contesto, quindi, trovare soluzioni che non danneggino (e anzi possano favorire) questi asset appare una sfida importante.

Essendo questa parte della ricerca particolarmente lunga, si fornisce in introduzione al capitolo un breve sunto delle principali informazioni racchiuse nei prossimi paragrafi. Ognuno di essi si concentra su un particolare aspetto del mercato locativo bolognese. Dopo una breve descrizione della città dal punto di vista socio economico e una carrellata sulle suddivisioni spaziali disponibili utilizzate in seguito, si procede ad analizzare - nell'ordine - i prezzi al metro quadro, i prezzi per abitazione, la sostenibilità degli affitti (per la famiglia mediana e per le fasce in difficoltà economica), la profittabilità degli affitti, il mercato locativo studentesco e quello dell'affitto breve a scopo turistico.

\section{Bologna e le sue zone}

389mila abitanti, una densità abitativa superiore ai 2'400 residenti per chilometro quadrato, una popolazione in crescita - nonostante un saldo naturale negativo - grazie alla presenza di oltre 50 mila residenti stranieri, un reddito medio vicino ai 25 mila euro (ma attorno ai 19mila se ci si riferisce al valore mediano). Questa, per sommi capi, la carta d'identità del comune di Bologna. Per studiare il mercato locativo bolognese, però, non si può considerare la città nel suo insieme, bensì occorre ricorrere a ripartizioni del territorio comunale che consentano di cogliere le differenze nel dettaglio. In questa ricerca viene dunque introdotta la suddivisione in tre fasce, determinata dagli accordi di applicazione del canone concordato ("Zona di Pregio", "Zona A", "Zona B"), seguita poi dalle 34 aree omogenee disegnate dall'OMI. Questi due tracciati, tuttavia, non sempre riportano fedelmente le distinzioni all'interno della città rispetto a variabili rilevanti come il reddito medio o la densità di abitanti, qui analizzate. Ciò avviene in particolar modo per le zone di applicazione del canone, dove la Zona B risulta essere dall'analisi eccessivamente ampia, comprendendo al suo interno quartieri popolari e aree rurali, settori relativamente poveri ed altri con redditi medi, non distanti da quelli registrati in prossimità del centro storico.

\section{II mercato locativo residenziale e abitazioni a canone concordato}

Secondo le stime effettuate sulla base del censimento ISTAT 2011 e dei dati catastali relativi allo stesso anno, il mercato locativo bolognese è caratterizzato da un potenziale "eccesso di domanda" di circa 6'000 famiglie. Per quanto questi conti siano basati su numerose ipotesi (e la stima si basi su un incrocio di informazioni censuarie del 2011), risulta evidente come tale mercato sia caratterizzato da una domanda sostenuta, determinata da un maggior numero di persone in cerca di affitto rispetto al totale delle abitazioni offerte sul mercato (ovvero da prezzi di mercato particolarmente elevati). Date le finalità della ricerca, si è ritenuto fondamentale 
indagare nel dettaglio la questione abitativa soffermandosi sulla tipologia contrattuale che, per antonomasia, è stata pensata al fine di permettere l'incontro tra domanda e offerta locativa delle famiglie: il contratto a canone concordato.

Per studiare approfonditamente la questione è stato costruito un database utilizzando i dati posseduti dal Comune relativamente ai contratti di locazione a canone concordato in essere negli anni 2010, 2012 e 2014 nel territorio bolognese. In termini numerici si dispone di 20'988 abitazioni nel 2010, 20'588 nel 2012 e 25'345 nel 2014. A fronte di una minima differenza tra i primi due anni, nel 2014 si assiste ad un netto incremento del numero di contratti in essere (+23.09\%). Tale aumento è probabilmente il risultato delle politiche adottate per favorire l'accesso ad unità abitative che hanno permesso una riduzione della distanza tra domanda e offerta. Da un lato l'accordo territoriale stipulato nel 2013 ha ridotto i prezzi massimi e minimi applicabili nei contratti a canone concordato, rendendo più sostenibili gli affitti per le famiglie; dall'altro, lo sconto fiscale legato alla riduzione della cedolare secca al $10 \%$ a partire dal 2014 rende, per i proprietari, maggiormente competitiva tale tipologia contrattuale rispetto alle locazioni a libero mercato, nonostante i prezzi maggiori di locazione di questi ultimi.

\section{I prezzi degli affitti al metro quadro}

Studiare l'andamento dei valori al metro quadro consente di mettere in luce il differente meccanismo di definizione dei prezzi. II canone concordato applica parametri crescenti all'aumentare della metratura e delle cosiddette caratteristiche di pregio dell'abitazione e decrescenti all'aumentare del numero dei vani che compongono l'abitazione stessa.

Nel 2013 i nuovi accordi sul canone concordato hanno sancito un calo vicino al 10\% dei valori al metro quadro, più sensibile nei parametri massimi. Ancora più intenso il calo dei prezzi di mercato: nel 2017, al termine della serie storica considerata, i valori risultano decisamente inferiori rispetto al 2009: $-16 \%$ per i minimi e $-22 \%$ per i massimi. Ad accentuare la caduta di questi ultimi ha contribuito proprio la variazione del canone concordato - d'altronde oltre metà ( e più ) degli affitti a Bologna applicano tale regime contrattuale: lo testimoniano una correlazione alta tra i rispettivi valori e la caduta dei prezzi di mercato tra 2013 e, soprattutto, 2014. Ad oggi, il combinato disposto tra calo sensibile dei prezzi al metro quadro sul libero mercato e diversa modalità di definizione dei prezzi, porta anche ad esiti contraddittori rispetto alle finalità del canone concordato stesso.

\section{Gli affitti mensili a Bologna e nelle sue zone}

Riscontrato il calo dei valori al metro quadro, la stima degli affitti mensili deve tenere conto delle superfici delle abitazioni incluse nella nostra popolazione, pressoché 
costanti nonostante la variazione degli stock di riferimento, tra le grandi metrature nella fascia pedecollinare e in parte del centro storico, e quelle inferiori in molti quartieri popolari. Mentre la collocazione dell'abitazione influisce similmente su affitti a canone concordato e a canone di mercato, che calano allontanandosi dai colli e dal centro verso le periferie, gli affitti a canone concordato attenuano fortemente gli effetti dell'aumento delle superfici e dei vani. L'effetto appena citato rende dunque i contratti a canone concordato sempre più convenienti per le abitazioni medie e grandi ma, di contro, rende gli affitti più alti rispetto al mercato per i monolocali e, in parte, i bilocali. Infine, introducendo le soglie di sostenibilità per i nuclei familiari (l'affitto non deve superare il $30 \%$ del reddito complessivo familiare), il canone concordato risulta alla loro portata nella Zona $B$ e, anche se in misura minore, nella Zona $A$; più complessa la situazione nella costosa Zona di Pregio, mentre paiono chiudersi molte porte per i nuclei mono-personali (o monoreddito), costretti a spingersi in periferia.

\section{Concorrenzialità e sostenibilità per il nucleo bolognese mediano}

Affinché un contratto d'affitto venga stipulato è necessario che si verifichi una duplice soddisfazione tra proprietario e inquilino. Per soddisfare la domanda deve essere rispettata la sostenibilità del canone mensile, definendo sostenibile una spesa per l'affitto inferiore al $30 \%$ del reddito netto. La sostenibilità viene valutata in funzione della famiglia bolognese mediana, considerando rispettivamente nuclei di una, due, tre e quattro persone. Questa, però, non è condizione sufficiente: affinché si determini una stipula del contratto è necessario che anche il locatore decida di offrire il proprio immobile sul mercato dei canoni concordati. L'offerta è valutata in funzione della concorrenzialità del canone concordato rispetto al canone libero di mercato: generalmente i canoni mensili concordati sono inferiori rispetto al mercato, ma questa tipologia contrattuale può risultare vantaggiosa grazie ai diversi regimi fiscali applicati.

La combinazione di concorrenzialità e sostenibilità determina diverse categorie all'interno delle quali sono classificate le abitazioni della banca dati. Il numero di abitazioni sostenibili e concorrenziali, e quindi effettivamente locabili a canone concordato, aumenta nel 2014 rispetto agli anni precedenti, attestandosi al $25 \%$ circa dell'intero stock. Queste abitazioni, però, sono perlopiù di dimensioni ridotte (superficie media $52.18 \mathrm{mq}$ e numero medio di vani 1.76, rispetto alla media cittadina di circa $80 \mathrm{mq}$ e 3.32 vani - valori 2014) e non adatte, quindi, alle famiglie più numerose.

Analizzando questo aspetto anche all'interno delle singole zone di applicazione del canone concordato si nota come, fatte salve le ipotesi e sempre con riferimento al 2014 , la percentuale di abitazioni ottimali sia maggiore per la zona di pregio (31.42\%) rispetto alla zona $A(28.25 \%)$ e alla $B(20.31 \%)$. Le percentuali più basse registrate per le zone $\mathrm{A}$ e $\mathrm{B}$ sono determinate dalla minor concorrenzialità, come dimostrano le 
quote delle categorie residue (le abitazioni sostenibili ma non concorrenziali aumentano passando da zona di pregio a zona B).

\section{Sostenibilità e concorrenzialità per i nuclei in difficoltà abitativa}

Lo stesso discorso relativo a concorrenzialità e sostenibilità è sviluppato anche in funzione delle famiglie in difficoltà economica, ossia quelle che hanno presentato domanda per l'edilizia residenziale pubblica ma non sono sufficientemente ben posizionate in graduatoria per accedere ad un immobile nei prossimi 3 anni. In questo caso, non essendo possibile definire soglie differenti in base alla composizione del nucleo familiare, vengono definiti due valori limite. Quello inferiore è il 30\% del reddito medio degli individui esclusi appartenenti al primo quartile, quello superiore il $30 \%$ di quello degli individui del quarto quartile. Le soglie così ottenute permettono di sostenere un canone mensile di $68.74 €$ per il valore minimo e di $495.31 €$ per quello massimo. Il valore superiore è maggiore rispetto al reddito mediano della famiglia bolognese unipersonale e permette di sostenere alcuni affitti. Tale sostenibilità, però, è registrata per le abitazioni di piccole dimensioni (monolocali e bilocali). Poiché le famiglie escluse dall'edilizia residenziale pubblica, ma che ad essa si rivolgono, sono generalmente numerose, l'offerta presente sul mercato del canone concordato appare inadatta a soddisfare le esigenze di queste persone. La situazione è ancora più critica se si considera che per la soglia minima, ossia per gli individui più poveri di questo gruppo, nessuna abitazione risulta sostenibile.

L'offerta del mercato del canone concordato bolognese è inadatta a soddisfare le esigenze dei nuclei più deboli e la causa principale va ricercata nella sostenibilità: da un lato i redditi di queste famiglie sono troppo bassi, dall'altro i canoni di locazione sono troppo elevati. Se in termini economici per altri beni tale problema potrebbe essere marginale, la casa, assieme all'alimentazione, rientrano nei beni fondamentali per la sopravvivenza e quindi il dato appare particolarmente allarmante.

\section{La domanda di alloggio degli studenti}

Sono stabilmente oltre trentamila gli universitari fuorisede che ogni anno devono trovare un alloggio in città (35'000 nell'ultimo anno) cui vanno aggiunti studenti, ricercatori e visiting professor impegnati in programmi di scambio dall'estero. II tutto accettando l'italiana peculiarità per la quale i cosiddetti studenti “in sede" (altri 30'000 circa) continuino a vivere con le famiglie. Questa mole di iscritti è la principale componente omogenea della domanda di alloggi sul mercato locativo di Bologna, anche a causa di un numero ridotto di posti negli studentati (ad oggi $1^{\prime} 600$ ) e nei collegi privati (peraltro frequentemente molto costosi).

Gli accordi di canone concordato prevedono una tipologia di contratto apposita, le cui condizioni risultano più vantaggiose rispetto ad un normale contratto residenziale: 
consente accordi di breve durata, garantendo al proprietario la possibilità di rientrare in possesso dell'immobile in tempi rapidi; permette di ospitare più studenti contemporaneamente, aumentando i margini di guadagno (ovvero riducendo il rischio di morosità ripartendolo su più famiglie); gode della tassazione agevolata al $10 \%$.

Assunto che ogni studente paga in media $350 €$ per una camera singola in appartamento condiviso, un proprietario potrà applicare i prezzi al metro quadro più alti consentiti dal canone, che in Zona di Pregio non sarebbero sostenibili dalle famiglie, trovando pressoché sempre redditizia risposta al proprio annuncio di locazione. Questo meccanismo di potenziale esclusione dei residenti, aggravato da potenziali pratiche illecite (l'utilizzo dei canoni concordati per coprire una cospicua parte in nero o di sub-affitto),$^{10}$ e alle recenti innovazioni nel calcolo delle tasse universitarie (il passaggio all'ISEE nello specifico), fanno di questo mercato un fenomeno da monitorare approfonditamente.

\section{II mercato turistico}

Con le presenze turistiche a Bologna, da alcuni anni in costante crescita, l'offerta ricettiva in città ha visto il fiorire del mercato degli affitti a breve termine attraverso I'utilizzo di piattaforme di home-sharing come Airbnb ${ }^{\text {TM }}$.

Ciò avviene all'interno di un quadro legislativo di difficile interpretazione ${ }^{11}$ che cerca di normare un settore sorto in breve tempo: prova ne sono le sole 382 strutture ricettive ritenute compatibili con il "modello Airbnb" ${ }^{\text {TM" }}$ registrate presso il Comune di Bologna, contro le oltre 3000 offerte uniche attualmente presenti sul portale stesso. Negli ultimi due anni gli annunci su Airbnb ${ }^{\mathrm{TM}}$ sono pressoché raddoppiati; nel 2017 hanno generato ricavi per oltre 27 milioni di euro (+66\% rispetto all'anno precedente). In questo contesto, va rimarcato l'accordo tra la start-up dell'home-sharing e Comune per la riscossione automatica della tassa di soggiorno, per scongiurare l'elusione e meglio monitorare il settore.

Peraltro, in un mercato così fiorente è presto emerso il fine imprenditoriale: quasi metà delle offerte sono pubblicate da proprietari che controllano più annunci, fino ad

\footnotetext{
${ }^{10}$ Nonostante oggi sia assolutamente più complesso affittare in nero rispetto ad alcuni anni fa, fatti di cronaca anche recenti indicano la necessità di non sottovalutare tale componente: http://www.bolognatoday.it/economia/case-affitto-nero-bologna-agente-immobiliareabusivo.html http://www.la7.it/lariadestate/video/bologna-la-giungla-degli-affitti-in-nero-agli-studenti-31-082016-192228

https://www.ilfattoquotidiano.it/2014/02/28/affitti-in-nero-famiglia-proprietaria-di-117immobili-evade-3-milioni-di-euro/897138/

11 La difficoltà interpretativa riguarda quantomeno il singolo cittadino che volesse intraprendere questo tipo di attività.
} 
arrivare al caso estremo della società Halldis Spa che controlla 76 "case intere" a Bologna.

A fronte degli indubbi benefici che la città riceve da tale flusso, nell'interesse della ricerca, la questione da monitorare è la riallocazione di unità abitative al mercato turistico a breve termine. Destinare una abitazione a questo mercato, sottraendola da quelle disponibili per normali affitti residenziali, è decisamente più conveniente, non solo per la flessibilità nella gestione degli annunci (disattivabili a piacimento e senza costi di entrata), ma soprattutto per gli enormi ricavi se paragonati all'affitto a lungo periodo. Nel 2017 una "casa intera" con un prezzo medio e occupata per 232 giorni I'anno (dato mediano per la città) può arrivare a fruttare, al netto delle tasse, da tre a cinque volte un affitto medio a canone concordato. Margini talmente ampi da consentire teoricamente, a parità di flussi turistici, un trasferimento di oltre 1'700 ulteriori abitazioni dal mercato residenziale a quello turistico, garantendo comunque a tutti gli affittuari Airbnb ${ }^{\text {TM }}$ entrate superiori al massimo ottenibile con un normale contratto d'affitto a canone di mercato. 


\section{Bologna e le sue zone}

Al 30 novembre 2017, Bologna conta 389'625 abitanti, settimo comune italiano per numero di residenti. L'andamento demografico della città negli anni di interesse di questa ricerca è rappresentato nella sottostante Figura 11. Si può notare in particolare un deciso incremento dei cittadini bolognesi tra il 2007 e il 2012 prima e tra il 2013 e 2017 poi, in ragione dei quali la popolazione risulta aumentata di circa il $4.3 \%$ su tutto il periodo (con un saldo positivo superiore alle 17 mila unità). Aumenta conseguentemente anche la densità abitativa in città, superando a fine periodo la soglia di 2400 abitanti per chilometro quadrato (2422 ab./ $\mathrm{km}^{2}$ nel 2017, per la precisione).

Figura 11. Popolazione residente a Bologna al 31 dicembre e densità abitativa (abitanti/(km²).

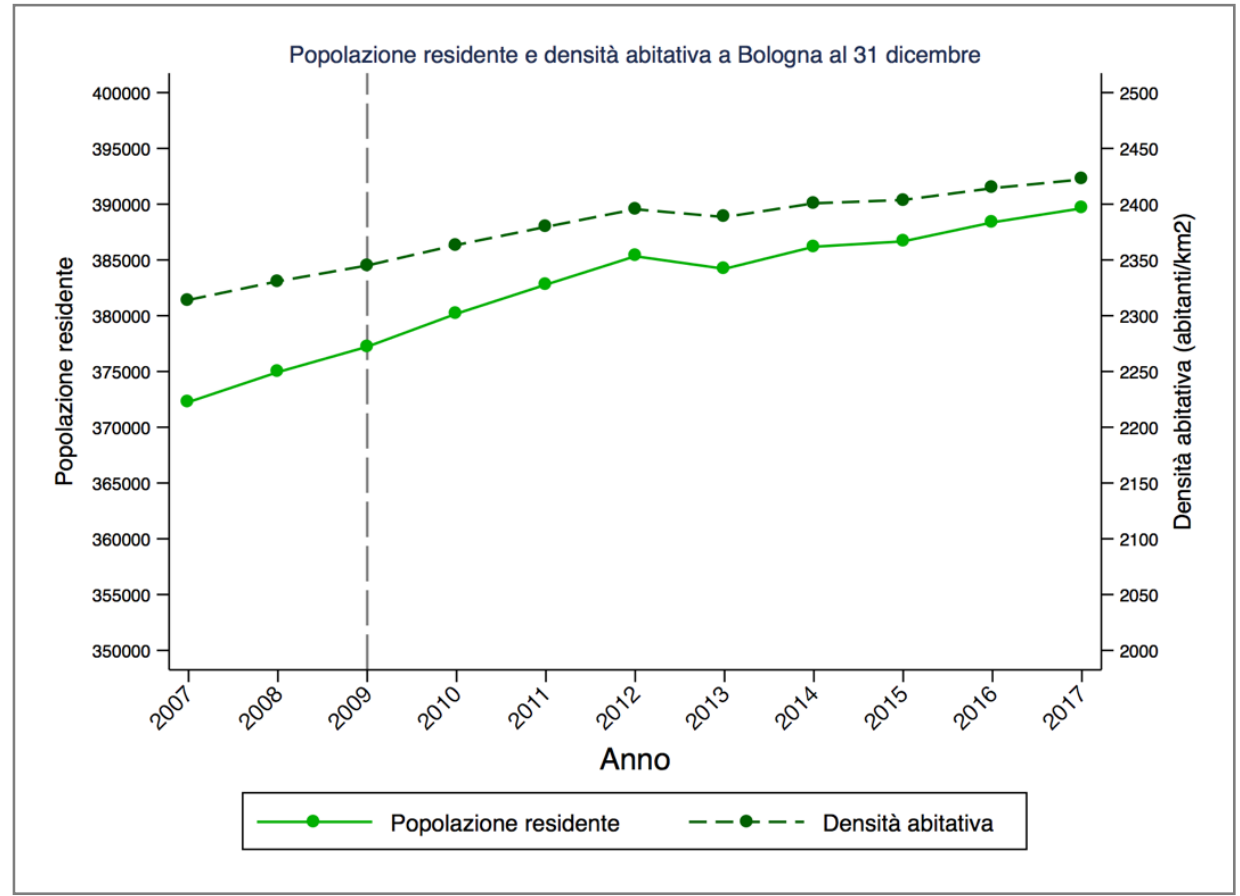

Per ciò che concerne i nuclei familiari, al 2017 erano 206'456, ricalcando la tendenza registrata per il totale dei residenti, con un aumento di oltre 12 mila famiglie a partire dalle 194'708 di dieci anni prima, concentrato prevalentemente nella prima metà del periodo considerato. Come rivela la Tabella 5, questo incremento della popolazione avviene nonostante un saldo naturale (ossia lo scarto tra nuovi nati e defunti) stabilmente in negativo: il tasso di natalità infatti registra un rapporto di 8 nascite ogni 1'000 abitanti nel 2016 mente quello di mortalità raggiunge i 12 decessi ogni 1'000 residenti. A determinare l'aumento della popolazione residente a Bologna contribuiscono invece i saldi migratori, sia interno (ossia da altri comuni italiani) che estero. In particolare, è interessante osservare l'andamento del primo per notare 
un'inversione dei flussi: fino al 2008 il capoluogo emiliano cedeva popolazione verso altri comuni italiani, mentre dal 2009 in poi la dinamica si è invertita sensibilmente. II crollo del 2007-2008 e il periodo di stagnazione dei 3 anni successivi sono facilmente imputabili agli effetti della crisi recessiva del 2007 e al netto calo di iscrizioni di cui ha sofferto l'Università nei primi anni della stessa. II saldo migratorio da paesi esteri rimane positivo in tutti gli anni considerati, benché dopo il picco raggiunto nel 2008, lo scarto tra nuovi iscritti e cancellati diminuisca fino ai 2'263 del 2016. Conseguentemente, il numero di cittadini stranieri residenti pressoché raddoppia nei dieci anni considerati, superando nel 2016 la soglia dei 58mila (dunque il 15\% della popolazione totale).

Tabella 5. Bilancio demografico del comune di Bologna. Fonte: ISTAT.

\begin{tabular}{|c|c|c|c|c|c|c|c|c|c|}
\hline & $\begin{array}{l}\frac{0}{0} \\
\frac{0}{N} \\
\frac{0}{0} \\
\frac{0}{0} \\
0\end{array}$ & 을 를 & 든 흘 & 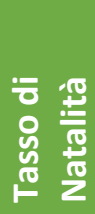 & 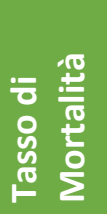 & 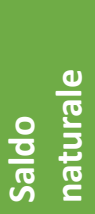 & 응 홍 을 을 & 응 을 을 율 & 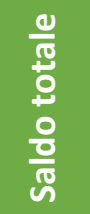 \\
\hline 2007 & 372256 & 194708 & 33602 & 8.1 & 12.8 & -1754 & -2294 & 3590 & -770 \\
\hline 2008 & 374944 & 197808 & 39480 & 8.1 & 12.9 & -1807 & -899 & 4914 & 2688 \\
\hline 2009 & 377220 & 200058 & 43664 & 8.4 & 12.7 & -1588 & 374 & 2821 & 2276 \\
\hline 2010 & 380181 & 202684 & 48466 & 8.2 & 12.3 & -1537 & 535 & 3204 & 2961 \\
\hline 2011 & 382784 & 204598 & 52473 & 8.2 & 12.5 & -1626 & 879 & 2029 & 2603 \\
\hline 2012 & 385329 & 206244 & 52473 & 8.0 & 12.6 & -1762 & 1694 & 3493 & 2545 \\
\hline 2013 & 384202 & 204681 & 56155 & 8.2 & 11.9 & -1428 & 2116 & 2551 & -1127 \\
\hline 2014 & 386181 & 205520 & 56302 & 8.6 & 11.8 & -1259 & 1681 & 2395 & 1979 \\
\hline 2015 & 386663 & 205199 & 57979 & 8.3 & 13.1 & -1837 & 1181 & 2391 & 482 \\
\hline 2016 & 388367 & 206050 & 58873 & 8.0 & 12.0 & -1540 & 2003 & 2263 & 1704 \\
\hline
\end{tabular}

Secondo quanto riportato nella Tabella 6, il reddito medio imponibile ai fini IRPEF registrato a Bologna, in riferimento alle dichiarazioni dei redditi 2015, si attesta sui 25 mila euro pro-capite, al di sopra della media nazionale di 20.690 euro. Alla rappresentazione della serie storica dei valori nominali, si affianca la serie storica del reddito medio (e mediano) rivalutato secondo il coefficiente dell'Indice dei prezzi al consumo ISTAT. 
Tabella 6. Reddito imponibile IRPEF a Bologna; dato nominale e rivalutato secondo i coefficienti dell'Indice dei prezzi al consumo per le famiglie di operai e impiegati ISTAT. Valore medio e mediano. Anni 2009-2015. ${ }^{12}$

\begin{tabular}{|c|c|c|c|c|c|}
\hline Anno & $\begin{array}{l}\text { Reddito } \\
\text { medio } \\
\text { nominale } \\
\text { (€) }\end{array}$ & $\begin{array}{l}\text { Reddito } \\
\text { mediano } \\
\text { nominale } \\
\text { (€) }\end{array}$ & $\begin{array}{l}\text { Coefficiente } \\
\text { di } \\
\text { rivalutazione } \\
\text { ITALIA }\end{array}$ & $\begin{array}{l}\text { Reddito } \\
\text { medio } \\
\text { reale (€) }\end{array}$ & $\begin{array}{l}\text { Reddito } \\
\text { mediano } \\
\text { reale }(€)\end{array}$ \\
\hline 2009 & 23713 & 18270 & 1.088 & 25800 & 19878 \\
\hline 2010 & 23771 & 18332 & 1.071 & 25459 & 19634 \\
\hline 2011 & 23757 & 18458 & 1.043 & 24779 & 19252 \\
\hline 2012 & 23682 & 18734 & 1.012 & 23966 & 18959 \\
\hline 2013 & 23904 & 19261 & 1.001 & 23928 & 19280 \\
\hline 2014 & 24628 & 19408 & 0.999 & 24603 & 19389 \\
\hline 2015 & 24995 & 19557 & 1.000 & 24995 & 19557 \\
\hline
\end{tabular}

La successiva Figura 12 restituisce graficamente l'andamento dei redditi presentati nella tabella precedente.

Figura 12. Reddito imponibile ai fini Irpef dichiarato dai contribuenti bolognesi in serie storica (anni 2009-2015). Valore medio e mediano in migliaia di euro. ${ }^{13}$ II dato nominale è stato successivamente rivalutato moltiplicandolo per i coefficienti dell'Indice dei prezzi al consumo per le famiglie di operai e impiegati ISTAT.

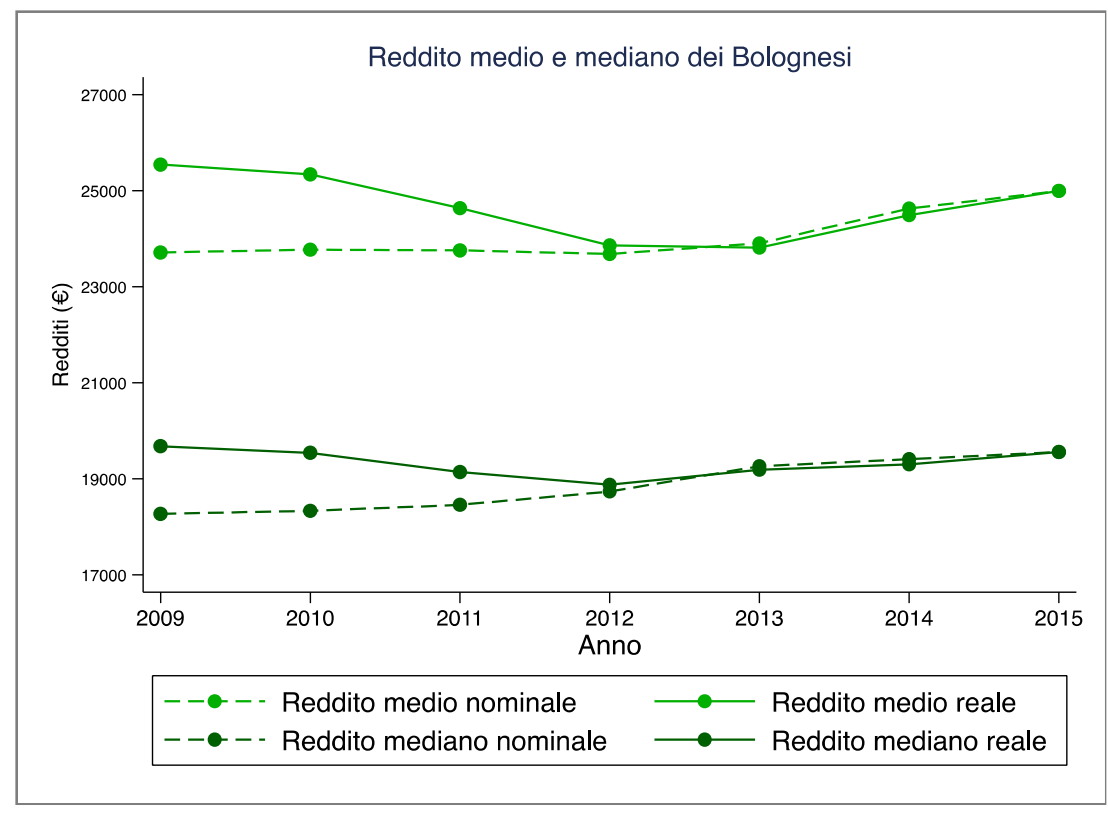

\footnotetext{
${ }^{12}$ Fonte per il reddito nominale: "I redditi 2015 dichiarati dalle cittadine e dai cittadini di Bologna", Comune di Bologna, Area Programmazione Controlli e Statistica, e precedenti.

${ }^{13}$ Fonte: "I redditi 2015 dichiarati dalle cittadine e dai cittadini di Bologna", Comune di Bologna, Area Programmazione Controlli e Statistica, e precedenti.
} 
Possiamo osservare l'effetto della rivalutazione nello scarto tra valori reali e nominali sui primi anni della serie: a fronte di un reddito nominale sostanzialmente stabile, il dato che tiene conto dei prezzi al consumo fa registrare un calo netto ( $-7 \%$ nei redditi medi reali) tra 2009 e 2012 - anni in cui l'impatto della crisi economica è risultato più forte - con una ripresa di entrambi a partire dal 2014.

Attenendoci alle annuali indagini ISTAT sulle "Spese per consumi delle famiglie", una quota rilevante del reddito dei nuclei viene destinato all'affitto dell'abitazione. Nei suoi report ISTAT stima la voce dei cosiddetti "affitti figurativi", ossia i costi da sostenere per la locazione di una abitazione identica per caratteristiche a quella in cui si vive. In particolare, nel 2016 quasi un quarto della spesa media mensile delle famiglie è destinata a questo capitolo (23,3\%). Giovandosi poi delle disaggregazioni territoriali, scopriamo che sono i nuclei del Centro Italia a dover destinare la quota di reddito maggiore $(28,8 \%)$, a fronte dei residenti nel Nord-Ovest che devono riservare poco più di un quinto del proprio budget mensile $(20,4 \%)$. Bologna infine ricade nella macro-area Nord-Est, dove la spesa per gli affitti figurativi è stimata pari al $24,4 \%$.

Figura 13. Prezzi medi mensili di locazione nelle prime dodici città italiane per numero di abitanti. Valori espressi in $€ / m q$, anno $2016^{14}$.

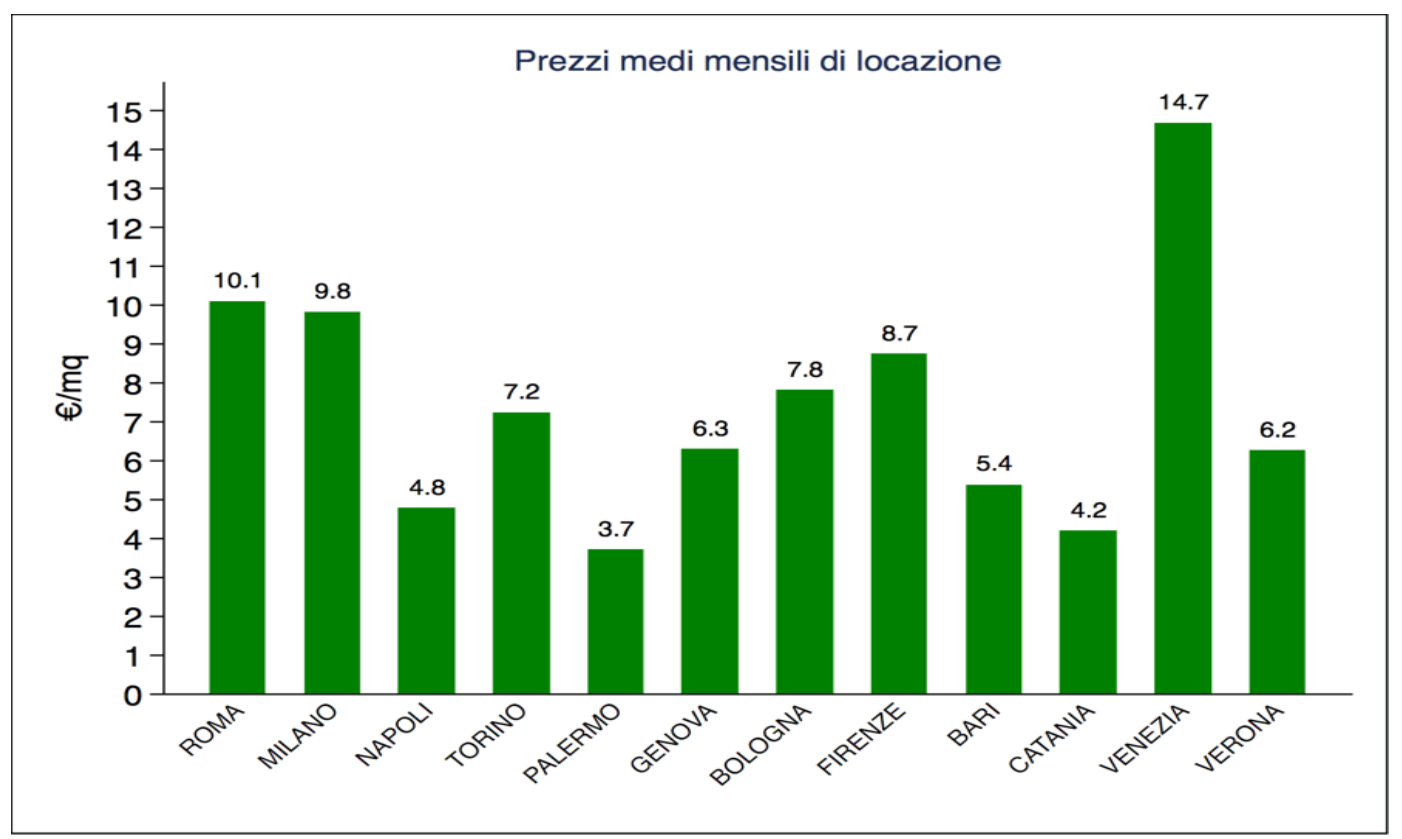

\footnotetext{
14 Il prezzo medio per Venezia risente della mancata rilevazione in numerose zone della città, tra cui in particolar modo quelle periferiche. Fonte: Quotazioni immobiliari OMI, secondo semestre 2016.
} 
Venendo dunque al mercato immobiliare, ed in particolar modo a quello degli affitti, su cui questo studio si concentra, la Figura 13 riporta i prezzi medi mensili relativi alla locazione di una abitazione a Bologna e nelle principali città italiane. I dati presentati, elaborati a partire dalle quotazioni dell'OMI ${ }^{15}$ nel secondo semestre del 2016, attribuiscono al capoluogo emiliano un canone mensile pari a 7,7 euro al metro quadro. Questo valore colloca Bologna sensibilmente alle spalle delle più grandi Milano e Roma e, in misura minore, di Firenze. D'altro canto, però, risulta particolarmente ampia la differenza nei prezzi di affitto tra Bologna stessa ed altri importanti centri come Torino e Genova al Nord e, ancor più, Napoli e Palermo a Sud.

\section{Bologna nelle sue 90 aree statistiche}

La descrizione di Bologna richiede una suddivisione della città in zone di dimensioni più circoscritte che aiutino a rendere conto delle differenze demografiche, economiche, sociali e abitative che la attraversano. Una prima possibile suddivisione di Bologna è quella nelle 90 aree statistiche tracciate dal Comune di Bologna nel 2004 (Figura 14 e la Tabella 7), che offre una ripartizione del territorio intermedia tra quella amministrativa in quartieri (9) o zone (18) e quella delle sezioni di censimento ISTAT (2'333).

\footnotetext{
15 I prezzi riportati nella Figura 13 sono ottenuti a partire dalle quotazioni OMI del secondo semestre 2016, per la locazione abitativa nelle città considerate. Sono state inclusi nel calcolo del prezzo medio soltanto i valori relativi a tipologie di edifici a finalità residenziale, nel loro stato prevalente.
} 
Figura 14. Mappa delle aree statistiche del comune di Bologna.

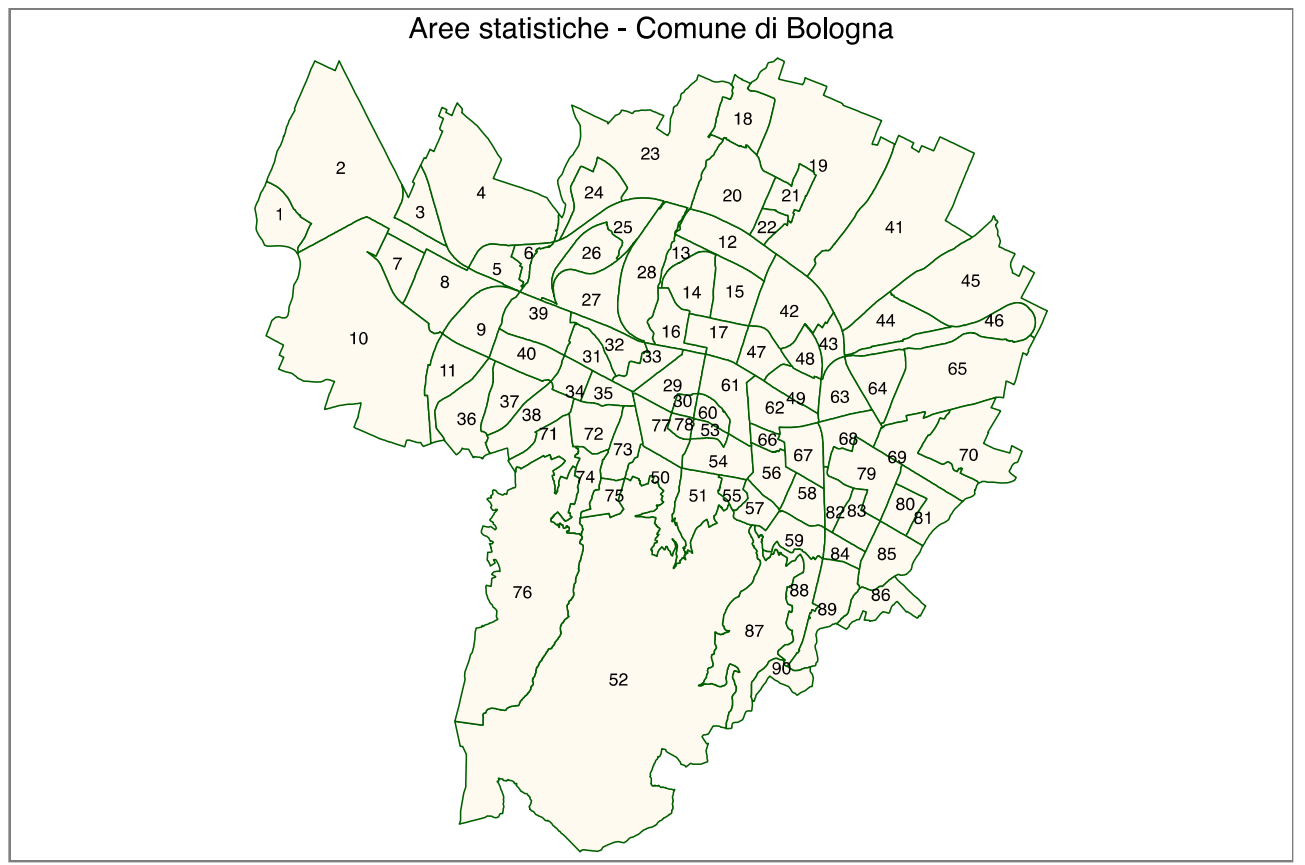

Rispetto alle 90 aree statistiche è possibile reperire informazioni sulle principali variabili di sfondo di nostro interesse: la popolazione, la densità abitativa, i redditi dichiarati, il numero di contribuenti.

Tabella 7. Elenco delle 90 aree statistiche del comune di Bologna.

\begin{tabular}{|l|l|l|l|l|l|}
\hline $\begin{array}{l}N^{\circ} \\
\text { area }\end{array}$ & Nome area & N & Nome area & N & Noa \\
\hline $\mathbf{1}$ & LAVINO DI MEZZO & $\mathbf{2 1}$ & MULINO DEL GOMITO & $\mathbf{4 0}$ & EMILIA PONENTE \\
\hline $\mathbf{2}$ & VIA DEL VIVAIO & $\mathbf{2 2}$ & LA DOZZA & $\mathbf{4 1}$ & $\begin{array}{l}\text { CADRIANO- } \\
\text { CALAMOSCO }\end{array}$ \\
\hline $\mathbf{3}$ & BARGELLINO & $\mathbf{2 3}$ & LAGHETTI DEL ROSARIO & $\mathbf{4 2}$ & FIERA \\
\hline $\mathbf{4}$ & AEROPORTO & $\mathbf{2 4}$ & LA NOCE & $\mathbf{4 3}$ & SAN DONNINO \\
\hline $\mathbf{5}$ & LA BIRRA & $\mathbf{2 5}$ & TIRO A SEGNO & $\mathbf{4 4}$ & PILASTRO \\
\hline $\mathbf{6}$ & LUNGO RENO & $\mathbf{2 6}$ & PESCAROLA & $\mathbf{4 5}$ & CAAB \\
\hline $\mathbf{7}$ & DUCATI-VILLAGGIO INA & $\mathbf{2 7}$ & LAZZARETTO & $\mathbf{4 6}$ & $\begin{array}{l}\text { SCALO MERCI SAN } \\
\text { DONATO }\end{array}$ \\
\hline $\mathbf{8}$ & BORGO CENTRO & $\mathbf{2 8}$ & BEVERARA & $\mathbf{4 7}$ & VIA DEL LAVORO \\
\hline $\mathbf{9}$ & TRIUMVIRATO-PIETRA & $\mathbf{2 9}$ & MARCONI-2 & $\mathbf{4 8}$ & MICHELINO \\
\hline $\mathbf{1 0}$ & RIGOSA & $\mathbf{3 0}$ & MARCONI-1 & $\mathbf{4 9}$ & VIA MONDO \\
\hline $\mathbf{1 1}$ & CASTELDEBOLE & $\mathbf{3 1}$ & $\begin{array}{l}\text { PRATI DI CAPRARA- } \\
\text { OSPEDALE MAGGIORE }\end{array}$ & $\mathbf{5 0}$ & OSSERVANZA \\
\hline $\mathbf{1 2}$ & CASERME ROSSE- & $\mathbf{3 2}$ & SCALO RAVONE & $\mathbf{5 1}$ & $\begin{array}{l}\text { SAN MICHELE IN } \\
\text { BOSCO }\end{array}$ \\
\hline $\mathbf{1 3}$ & CNR & $\mathbf{3 3}$ & ZANARDI & $\mathbf{5 2}$ & PADERNO \\
\hline $\mathbf{1 4}$ & ARCOVEGGIO & $\mathbf{3 4}$ & VELODROMO & $\mathbf{5 3}$ & GALVANI-1 \\
\hline $\mathbf{1 5}$ & VIA FERRARESE & $\mathbf{3 5}$ & VIA VITTORIO VENETO & $\mathbf{5 4}$ & GALVANI-2 \\
\hline & & & & & \\
\hline
\end{tabular}




\begin{tabular}{|l|l|l|l|l|l|}
\hline $\mathbf{1 6}$ & $\begin{array}{l}\text { EX MERCATO } \\
\text { ORTOFRUTTICOLO }\end{array}$ & $\mathbf{3 6}$ & $\begin{array}{l}\text { VILLAGGIO DELLA } \\
\text { BARCA }\end{array}$ & $\mathbf{5 5}$ & $\begin{array}{l}\text { GIARDINI } \\
\text { MARGHERITA }\end{array}$ \\
\hline $\mathbf{1 7}$ & PIAZZA DELL'UNITA' & $\mathbf{3 7}$ & BATTINDARNO & $\mathbf{5 6}$ & MEZZOFANTI \\
\hline $\mathbf{1 8}$ & SAN SAVINO & $\mathbf{3 8}$ & CANALE DI RENO & $\mathbf{5 7}$ & SIEPELUNGA \\
\hline $\mathbf{5 8}$ & DAGNINI & $\mathbf{6 9}$ & CROCE DEL BIACCO & $\mathbf{8 0}$ & DUE MADONNE \\
\hline $\mathbf{5 9}$ & CHIESANUOVA & $\mathbf{7 0}$ & STRADELLI GUELFI & $\mathbf{8 1}$ & LUNGO SAVENA \\
\hline $\mathbf{6 0}$ & IRNERIO-1 & $\mathbf{7 1}$ & STADIO-MELONCELLO & $\mathbf{8 2}$ & PONTEVECCHIO \\
\hline $\mathbf{6 1}$ & IRNERIO-2 & $\mathbf{7 2}$ & XXI APRILE & $\mathbf{8 3}$ & BITONE \\
\hline $\mathbf{6 2}$ & CIRENAICA & $\mathbf{7 3}$ & SAN GIUSEPPE & $\mathbf{8 4}$ & CAVEDONE \\
\hline $\mathbf{6 3}$ & SCANDELLARA & $\mathbf{7 4}$ & RAVONE & $\mathbf{8 5}$ & VIA ARNO \\
\hline $\mathbf{6 4}$ & VIA LARGA & $\mathbf{7 5}$ & VIA DEL GENIO & $\mathbf{8 6}$ & $\begin{array}{l}\text { OSPEDALE } \\
\text { BELLARIA }\end{array}$ \\
\hline $\mathbf{6 5}$ & ROVERI & $\mathbf{7 6}$ & SAN LUCA & $\mathbf{8 7}$ & MONTE DONATO \\
\hline $\mathbf{6 6}$ & $\begin{array}{l}\text { OSPEDALE } \\
\text { SANT'ORSOLA }\end{array}$ & $\mathbf{7 7}$ & MALPIGHI-2 & $\mathbf{8 8}$ & VIA TOSCANA \\
\hline $\mathbf{6 7}$ & MENGOLI & $\mathbf{7 8}$ & MALPIGHI-1 & $\mathbf{8 9}$ & CORELLI \\
\hline $\mathbf{6 8}$ & GUELFA & $\mathbf{7 9}$ & FOSSOLO & $\mathbf{9 0}$ & PONTE SAVENA-LA \\
\hline & & & & & BASTIA \\
\hline
\end{tabular}

La Figura 15 riporta la densità abitativa nelle 90 aree statistiche in cui è suddiviso il territorio di Bologna. Osservando la mappa, le cinque sfumature che colorano le zone corrispondono ai quintili della distribuzione, abbinando alla tonalità più chiara il $20 \%$ del campione a minore densità (espressa in abitanti $/ \mathrm{km}^{2}$ ), salendo progressivamente di tono e densità. A questo proposito, la successiva Tabella 8 contiene le principali statistiche descrittive delle cinque fasce adottate. L'area più densamente abitata risulta essere quella di "Bitone", sull'asse della via Emilia, ad est del centro storico, con un rapporto pari a $17^{\prime} 796$ abitanti per $\mathrm{km}^{2}$, dovuto alla presenza di oltre diecimila residenti in un'area contenuta $\left(0,6 \mathrm{~km}^{2}\right)$. Attorno ad essa si concentrano altre zone fortemente abitate, come "Pontevecchio", "Dagnini", "Mezzofanti", "Mengoli". Più in generale, un'alta densità si registra in buona parte del centro storico, nelle sue prossimità occidentali (zona "XXI Aprile", la più popolosa, "Velodromo", "Zanardi"), nei quartieri a nord della stazione ferroviaria ("Piazza dell'Unità", "Arcoveggio", "via Ferrarese", "via del Lavoro", "Via Mondo").

Di contro, sono le parti più periferiche del territorio comunale, dal settore collinare a sud, alle aree di prima campagna a nord-est e ad ovest oltre Borgo Panigale, a rivelare una minore densità abitativa. Occorre inoltre segnalare che, in virtù del disegno delle zone che tiene conto di specifici insediamenti e infrastrutture, alcune di esse non rispondono a funzioni abitative specifiche, dunque inevitabilmente mostrano una scarsa densità; tra questi, ad esempio: "Aeroporto", "Fiera", "Scalo merci San Donato", "CAAB" ed i poli ospedalieri. 
Figura 15. Densità abitativa nelle novanta aree statistiche della città di Bologna. Le aree sono raffigurate in diversi toni di verde a seconda del quintile della distribuzione di frequenza entro cui ricadono: a colori più intensi corrisponde una maggiore densità abitativa.

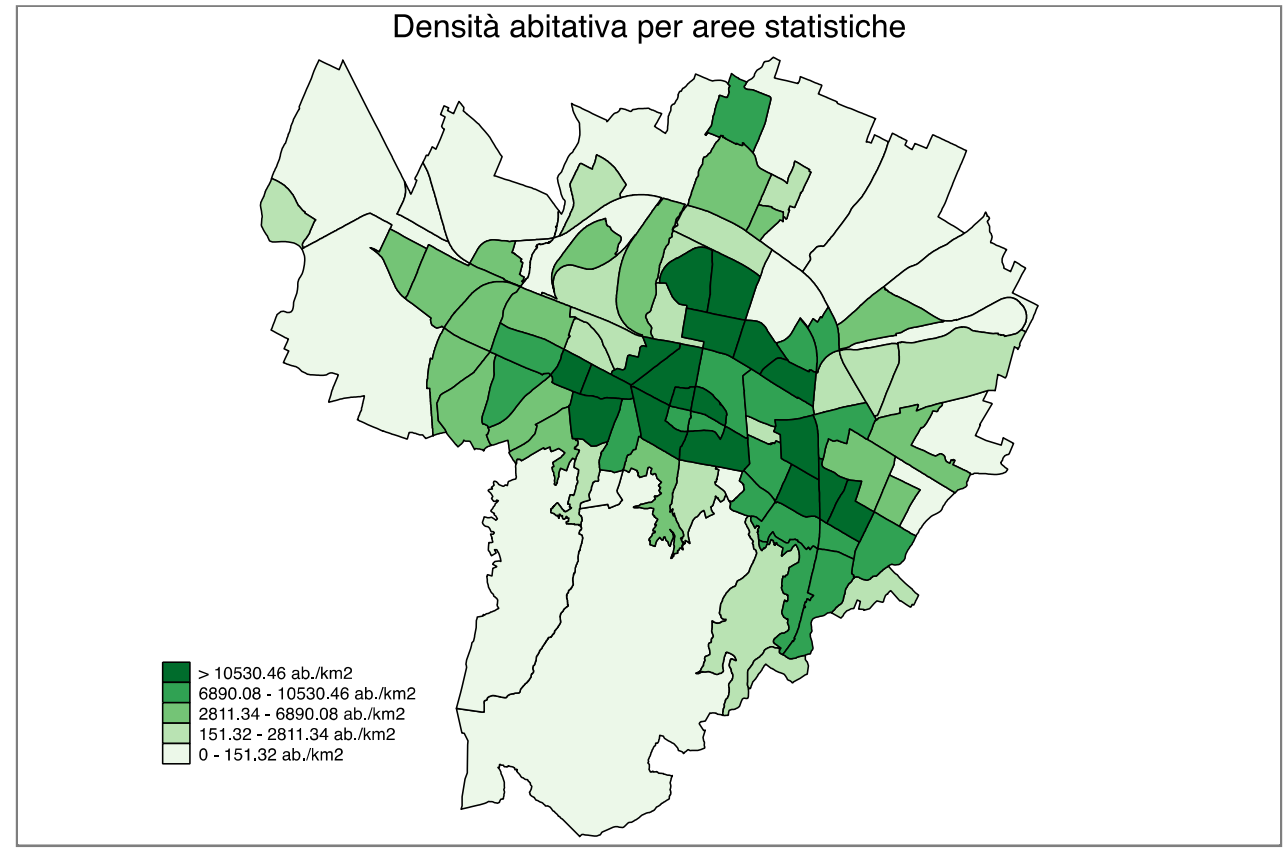

Tabella 8. Statistiche descrittive densità abitativa (abitanti/km2) per aree statistiche

\begin{tabular}{|l|r|r|r|}
\hline Quantile & Densità media & Densità minima & Densità massima \\
\hline $\mathbf{1}$ & 100.84 & 14.69 & 151.32 \\
\hline $\mathbf{2}$ & 1074.77 & 160.32 & 2811.34 \\
\hline $\mathbf{3}$ & 4634.18 & 2943.68 & 6890.08 \\
\hline $\mathbf{4}$ & 9116.22 & 7017.41 & 10530.46 \\
\hline $\mathbf{5}$ & 13189.37 & 11078.08 & 17796.40 \\
\hline
\end{tabular}

Prima di discutere i livelli di reddito nelle zone di Bologna, osserviamo la Figura 16 e la Tabella 9, relative alla densità di contribuenti all'interno delle aree statistiche, calcolata dividendo il numero di contribuenti ai fini IRPEF per l'ampiezza del territorio, espressa in $\mathrm{km}^{2}$. Ricordando che per la lettura delle differenti tonalità nella mappa si applica il criterio definito in precedenza per la densità abitativa, non possiamo che rimarcare la pressoché perfetta sovrapponibilità rispetto alla precedente Figura 15. 
Figura 16. Densità di contribuenti per area statistica (numero contribuenti / $\mathrm{km}^{2}$ ). Le aree sono raffigurate in diversi toni di verde a seconda del quintile della distribuzione di frequenza entro cui ricadono: a colori più intensi corrisponde una maggiore densità di contribuenti.

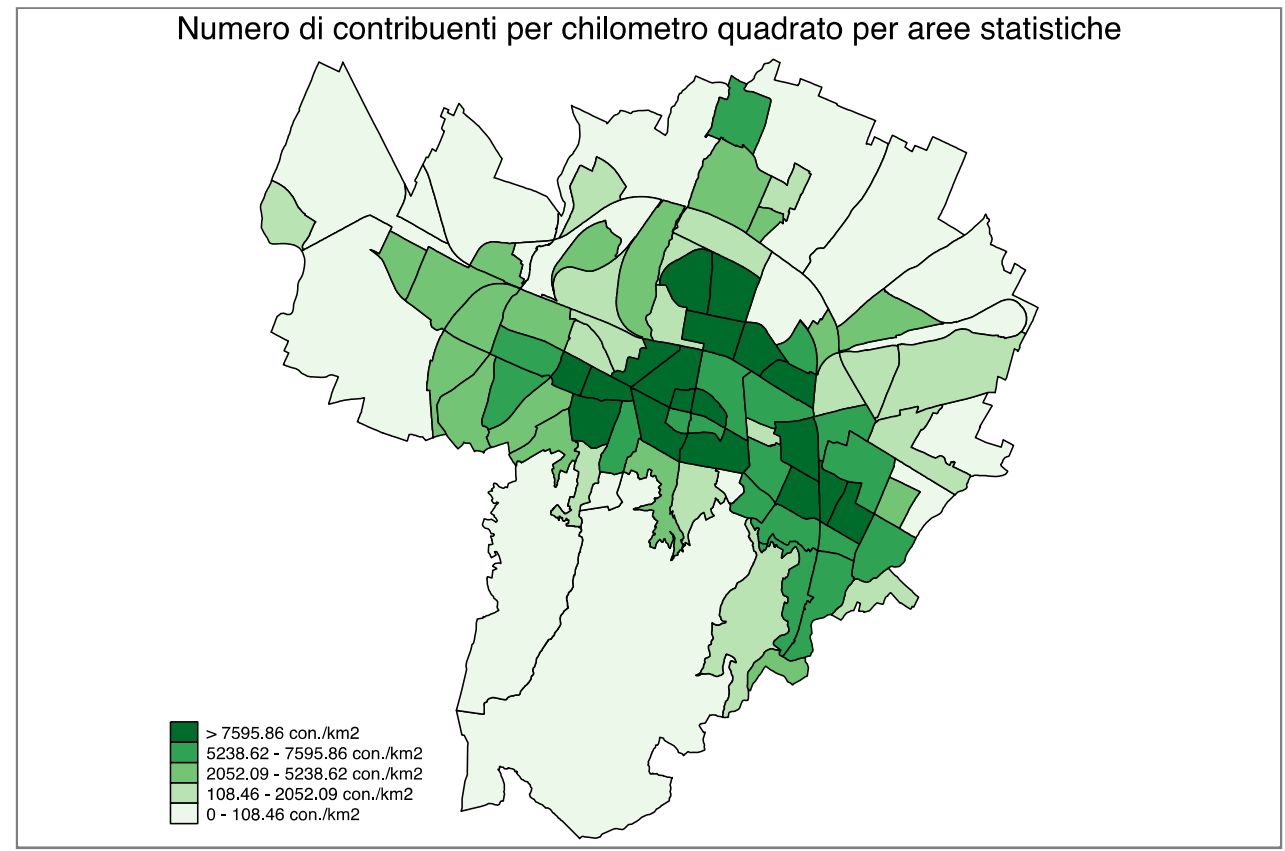

Da una densità media pari a 66 contribuenti per kilometro quadrato caratteristica delle zone a basso impatto residenziale si passa a 9638 contribuenti per kilometro quadrato nelle già citate aree fortemente abitate.

Tabella 9. Statistiche descrittive densità di contribuenti (contribuenti/km2) per aree statistiche.

\begin{tabular}{|l|r|r|r|}
\hline Quantile & Densità media & Densità minima & Densità massima \\
\hline $\mathbf{1}$ & 66.66 & 8.82 & 108.46 \\
\hline $\mathbf{2}$ & 718.75 & 109.36 & 2052.09 \\
\hline $\mathbf{3}$ & 3404.50 & 2079.72 & 5238.62 \\
\hline $\mathbf{4}$ & 6640.83 & 5354.13 & 7595.86 \\
\hline $\mathbf{5}$ & 9637.90 & 8019.70 & 13218.49 \\
\hline
\end{tabular}

Veniamo ora alla distribuzione del reddito nelle aree statistiche di Bologna, calcolato dividendo il reddito imponibile IRPEF totale, dichiarato all'interno delle singole zone, per il numero di contribuenti che le stesse annoverano. La Figura 17 e la relativa Tabella 10, contenenti le statistiche descrittive, evidenziano una distribuzione particolarmente diversificata, all'interno della quale il reddito medio per contribuente cresce gradualmente nei primi quattro quartili (dai $19^{\prime} 592.50$ euro del $20 \%$ più povero si sale progressivamente fino ai $26^{\prime} 350.80$ della seconda fascia più ricca), per poi raggiungere un valore oltre i 36 mila euro per il quintile più ricco. 
Figura 17. Distribuzione dei quintili di reddito nelle aree statistiche del comune di Bologna. II dato è ricavato dividendo il reddito imponibile IRPEF totale per il totale dei contribuenti della determinata zona. Le aree sono raffigurate in diverse tonalità a seconda del quintile della distribuzione di frequenza entro cui ricadono: a colori più intensi corrisponde maggior reddito medio.

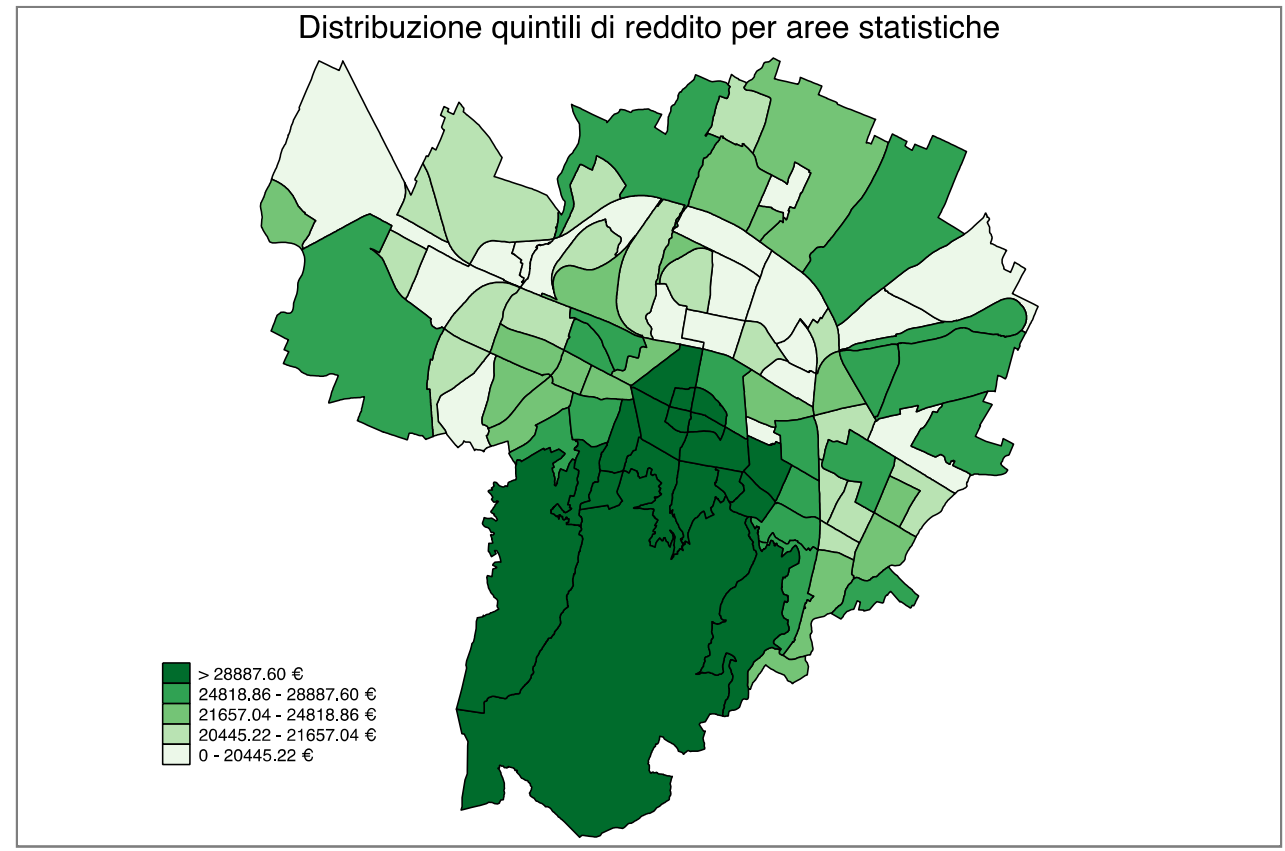

La Tabella 10 riporta il reddito medio nelle cinque fasce individuate, ponderato a seconda del peso demografico delle singole zone, in modo che eventuali outliers (rilevati in particolare nelle code della distribuzione) non incidano oltremodo sulla media di gruppo.

In generale i colori della mappa si fanno più intensi nella zona che va dal centro storico ai colli, a sud, ossia porzioni del territorio comunale densamente abitate soltanto nella parte urbana. Di contro, possiamo affermare che le condizioni meno agiate si registrano in particolare nei quartieri della prima periferia settentrionale, tra cui emergono- in quanto fortemente popolate- l'area di Piazza dell'Unità e circostanti, tra cui "Via Ferrarese", "Via Mondo" (dove il reddito medio per contribuente si attesta attorno ai ventimila euro); ad esse si affiancano altri quartieri popolari come il Pilastro, il "Villaggio della Barca", e Borgo Panigale. Da segnalare infine come, ai margini del territorio comunale, avvicinandosi alla prima campagna, si trovino alcune aree mediamente ricche, benché scarsamente abitate; tra queste citiamo "Rigosa" ad ovest, "Laghetti del Rosario" e "Cadriano-Calamoscio" a nord. 
Tabella 10. Statistiche descrittive per la distribuzione del reddito nelle aree statistiche. In corsivo tra parentesi la deviazione standard.

\begin{tabular}{|l|r|r|r|r|}
\hline Quintile & $\begin{array}{c}\text { Reddito medio } \\
\text { ponderato }(€)^{16}\end{array}$ & $\begin{array}{c}\text { Reddito } \\
\text { medio (€) }\end{array}$ & Reddito minimo (€) & Reddito Massimo (€) \\
\hline $\mathbf{1}$ & 19592.50 & 18703.48 & 13751.27 & 20445.22 \\
\hline $\mathbf{2}$ & 21144.07 & 21068.28 & 20452.81 & 21657.04 \\
\hline $\mathbf{3}$ & 23166.05 & 23080.99 & 21735.63 & 24818.86 \\
\hline $\mathbf{4}$ & 26350.80 & 26653.01 & 25320.96 & 28887.60 \\
\hline $\mathbf{5}$ & 36222.20 & 44289.87 & 28998.19 & 80118.10 \\
\hline
\end{tabular}

Le mappe del mercato immobiliare: zone OMI e zone di applicazione del Canone Concordato

Dopo aver fatto ricorso alle aree statistiche disegnate nel 2004 per offrire una breve descrizione di sfondo della città di Bologna nelle sue differenti zone, rispetto a variabili rilevanti come la densità abitativa e il reddito medio, occorre introdurre due nuove suddivisioni del territorio comunale, funzionali all'analisi del mercato immobiliare: le fasce di applicazione dei contratti a canone concordato e le aree omogenee tracciate dall'OMI dell'Agenzia delle Entrate.

Se le aree statistiche rappresentavano un reticolo coerente rispetto alla suddivisione della città in quartieri da un lato e in sezioni di censimento dall'altro, lo specifico ambito di applicazione delle due nuove mappe che ci apprestiamo ad utilizzare comporta un disegno dei loro confini non sovrapponibile alle prime.

In dettaglio, gli accordi di applicazione del canone concordato suddividono la città in tre fasce (Zona di pregio, Zona A e Zona B), mentre OMI individua 34 aree omogenee.

La Figura 18 sovrappone questi due differenti riferimenti territoriali, tracciando in nero i confini delle aree omogenee $\mathrm{OMI}$, mentre le più spesse linee rosse delimitano le zone di applicazione del canone concordato.

16 Il dato rappresenta la media dei redditi all'interno dei quintili, ponderata per il numero di contribuenti all'interno delle aree statistiche incluse nel relativo quintile. 


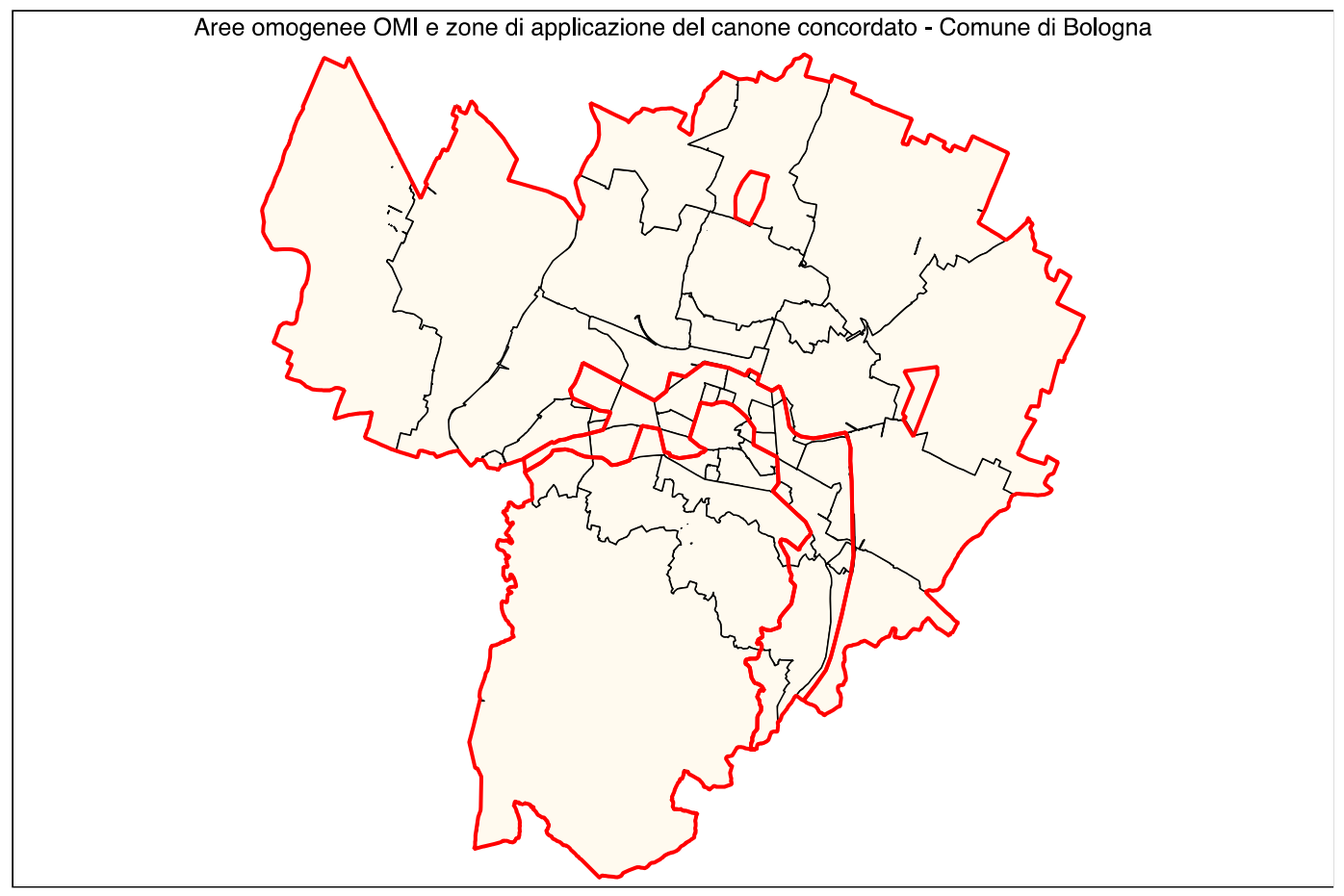

La zona di pregio racchiude le aree meridionali della città: dalla zona collinare fino a parte del centro storico. La parte restante del centro storico, insieme alle zone ad est di questo, ricadono nella zona A. Infine, la zona B copre la maggior parte del territorio cittadino, soprattutto per quel che riguarda le zone più abitate, comprendendo tutti $i$ terreni a Nord del centro storico. Confrontando poi questi valori con la distribuzione della ricchezza per aree statistiche (Figura 17), la zona di pregio racchiude tutte le aree più ricche della città. La Zona $A$ delimita aree statistiche con ricchezza medio-alta, mentre la zona B è abbastanza eterogenea. Quest'ultima zona, infatti, comprende le aree statistiche più povere del comune (quelle più vicine al centro) e aree caratterizzate da una maggior ricchezza, ossia alcune di quelle prossime ai confini comunali. Relativamente alla densità abitativa la zona di pregio è quella meno densamente popolata, insieme alla parte settentrionale della zona B. La restante parte di questa zona è mediamente popolata rispetto alle superfici di riferimento, anche se è la zona $\mathrm{A}$ che racchiude le aree statistiche più densamente popolate.

La Figura 19 considera soltanto le 34 aree omogenee $\mathrm{OMI}^{17}$, evidenziandone la sigla identificativa. Questo report farà riferimento a 32 di queste zone, poiché il dataset utilizzato non registra alcuna abitazione locata a canone concordato nell'area D6 -

\footnotetext{
17 Per un maggior dettaglio relativo alla suddivisione territoriale in zone OMI si rimanda alla nota metodologica.
} 
"Fiera"; inoltre, per una maggiore correttezza di calcolo dei valori di locazione, le zone R1 ed E5 sono state unite e considerate congiuntamente ${ }^{18}$.

La Tabella 11 riassume queste informazioni, riportando per ogni zona OMI, oltre al relativo nome per esteso, la collocazione all'interno delle fasce di applicazione di canone. Soltanto 15 delle 32 aree omogenee ricadono interamente in un' unica fascia. Tra le aree "miste" sono sei quelle comprese tra Zona di Pregio e Zona A, mentre sette sono suddivise tra Zona A e Zona B; infine, la zona D19, "Stadio - Funivia", presenta al suo interno tutte e tre le fasce di applicazione del canone.

Figura 19. Mappa delle zone omogenee OMI del comune di Bologna.

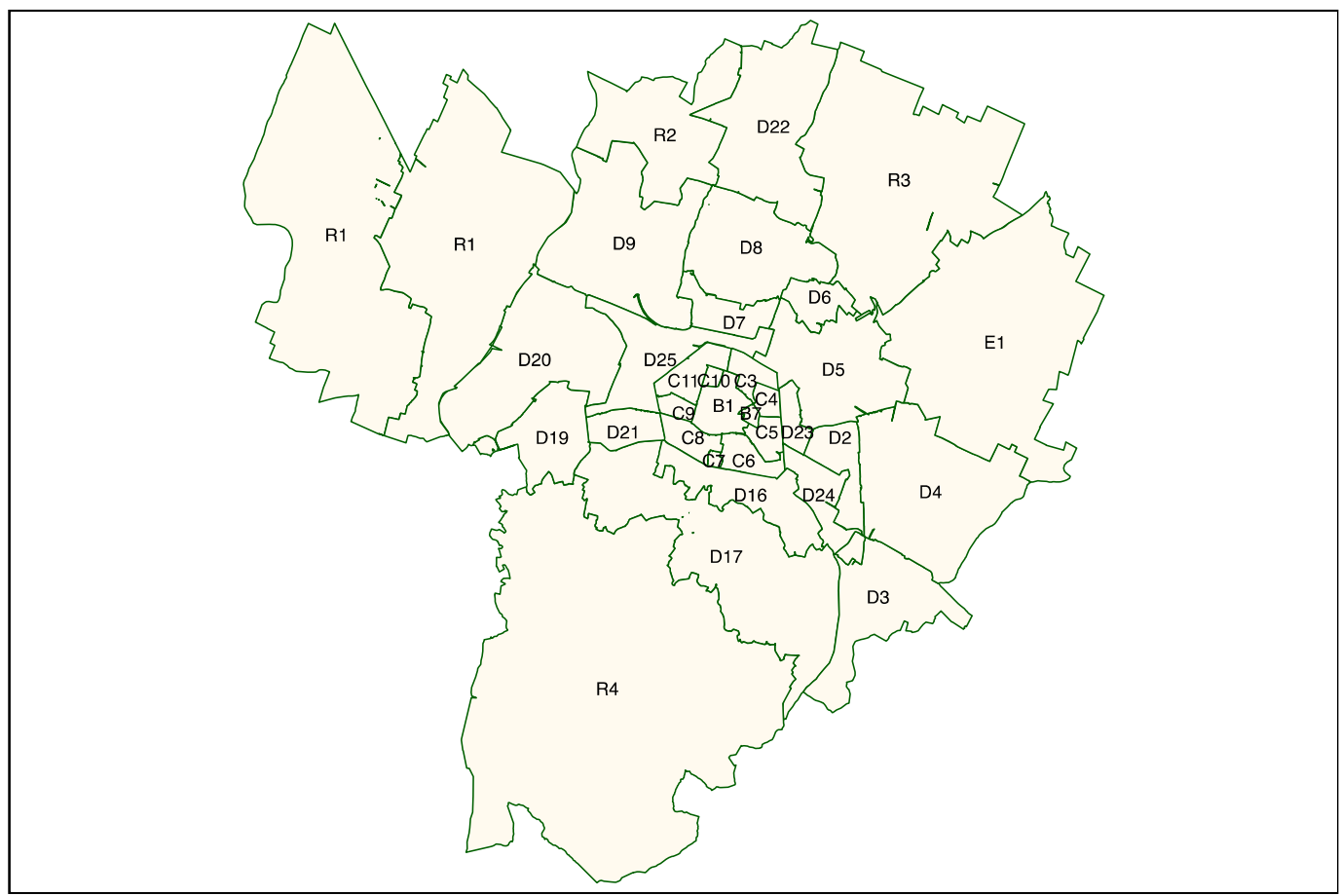

${ }^{18} \mathrm{Nel}$ report si farà riferimento alla somma di queste due zone con la sigla R1. 
Tabella 11. Prospetto delle Aree OMI di Bologna e relativa zona di canone concordato.

\begin{tabular}{|c|c|c|}
\hline $\begin{array}{c}\text { Sigla } \\
\text { Aree } \\
\text { OMI }\end{array}$ & Definizione Aree OMI & $\begin{array}{l}\text { Zone Canone } \\
\text { Concordato }\end{array}$ \\
\hline B1 & Centro Storico & PREGIO + A \\
\hline B7 & Zamboni - Casteltialto- Aldrovandi - San Vitale & PREGIO \\
\hline C10 & Quartiere Marconi Porto: Mille - Galliera - Riva Reno - Marconi & A \\
\hline C11 & $\begin{array}{l}\text { Quartiere Marconi: S. Felice - Grada - Vicini - Pietramellara - } \\
\text { Indipendenza - Falegnami - Galliera - Mille - Marconi }\end{array}$ & A \\
\hline C3 & $\begin{array}{l}\text { Irnerio - Centotrecento - Moline - Righi - P.zza VIII Agosto - } \\
\text { Indipendenza - V.li Masini e Pichat }\end{array}$ & A \\
\hline C4 & $\begin{array}{l}\text { Centotrecento - Irnerio - P.zza di Porta San Donato - V.le Filopanti - } \\
\text { San Vitale Petroni }\end{array}$ & A \\
\hline C5 & $\begin{array}{l}\text { San Vitale - Viale Ercolani - Piazza Carducci - Dante - Santo Stefano } \\
\text { - Guerrazzi - Piazza Aldrovandi }\end{array}$ & PREGIO + A \\
\hline C6 & $\begin{array}{l}\text { Quartiere Galvani: Santo Stefano - P.zza di Porta S. Stefano - Viali } \\
\text { Gozzadini e Panzacchi - Savenella Tovaglie - D’Azeglio - }\end{array}$ & PREGIO \\
\hline C7 & $\begin{array}{l}\text { Quartiere Galvani - Mura di Porta Castiglione: D’Azeglio - Tovaglie } \\
\text { Savenella - Mura di Porta Castiglione }\end{array}$ & PREGIO \\
\hline C8 & $\begin{array}{l}\text { Quartiere Malpighi Saragozza: Sant Isaia - Viali Pepoli Aldini - } \\
\text { D’Azeglio Barberia }\end{array}$ & PREGIO + A \\
\hline C9 & $\begin{array}{l}\text { Quartiere Malpighi: S. Felice - Grada - Vicini - S. Isaia - S. Francesco } \\
\text { - Malpighi }\end{array}$ & A \\
\hline D16 & Pedecollinare & PREGIO + A \\
\hline D17 & Pedecollinare e semicollinare & PREGIO + A \\
\hline D19 & Stadio - Funivia & $\begin{array}{l}\text { PREGIO + A + } \\
\text { B }\end{array}$ \\
\hline D2 & San Vitale & $\mathrm{A}$ \\
\hline D20 & Saffi - Santa Viola - Barca & $A+B$ \\
\hline D21 & Andrea Costa - Saragozza & PREGIO + A \\
\hline D22 & Corticella & $A+B$ \\
\hline D23 & V.le Filopanti - Massarenti - Zanolini - Stazione S. Vitale & $A+B$ \\
\hline D24 & Murri & A \\
\hline D25 & Stazione - Viale Filopanti - Via del Chiù & $A+B$ \\
\hline D3 & San Ruffillo Est & $A+B$ \\
\hline D4 & Mazzini - Fossolo & B \\
\hline D5 & San Donato & $A+B$ \\
\hline D6 & Fiera & B \\
\hline D7 & Matteotti - P.zza Unità & B \\
\hline D8 & Bolognina - Arcoveggio & B \\
\hline D9 & Marco Polo - Pescarola & B \\
\hline E1 & Roveri - Pilastro & $A+B$ \\
\hline R1 & $\begin{array}{l}\text { Agricola nord ovest (comprende Borgo Panigale - Birra- } \\
\text { Casteldebole) }\end{array}$ & B \\
\hline $\mathbf{R 2}$ & Agricola nord & B \\
\hline R3 & Agricola nord est & B \\
\hline R4 & Agricola sud & A \\
\hline
\end{tabular}




\section{II mercato locativo residenziale e abitazioni a canone concordato}

\section{Il mercato locativo residenziale del Comune di Bologna}

Al fine di comprendere le dinamiche del fenomeno locativo nel comune di Bologna è utile presentare una prima stima della domanda e dell'offerta di tale mercato. A tal fine ci si avvale di diverse fonti e sono necessarie alcune ipotesi. II mercato come sottolineato "è in equilibrio economico", nel senso che a tali prezzi l'offerta è tutta soddisfatta, tuttavia il fatto che il mercato abbia allocato le unità offerte non implica che tale risultato sia socialmente ottimale o desiderabile data la particolarità del bene casa. L'obiettivo in questo caso diventa quello di individuare una dimensione indicativa della differenza tra domanda e offerta, essendo impossibile quantificare esattamente la dimensione della domanda di alloggi in locazione non soddisfatta. Come riferimento vengono utilizzate le informazioni del Censimento ISTAT 2011 e le statistiche catastali elaborate da OMI, sempre del 2011, per avere un confronto temporale coerente.

Tabella 12. Famiglie in abitazione per titolo di godimento. Censimento ISTAT 2011.

\begin{tabular}{|l|r|r|r|r|r|}
\hline \multicolumn{5}{|c|}{ Famiglie in abitazione per titolo di godimento - Censimento 2011} \\
\hline Tipo di godimento & Proprietà & Affitto & \multicolumn{1}{c|}{ Altro } & \multicolumn{1}{c|}{ Totale } & $\begin{array}{c}\text { \% di Famiglie in } \\
\text { Affitto }\end{array}$ \\
\hline Italia & 17660769 & 4402786 & 2431774 & 24495329 & 17.97 \\
\hline Emilia-Romagna & 1365668 & 371169 & 173101 & 1909938 & 19.43 \\
\hline $\begin{array}{l}\text { Provincia Di } \\
\text { Bologna }\end{array}$ & 315911 & 104107 & 38719 & 458737 & 22.69 \\
\hline Comune di Bologna & 118720 & 57279 & 16574 & 192573 & 29.74 \\
\hline
\end{tabular}

La Tabella 12 presenta la suddivisione delle famiglie bolognesi per titolo di godimento dell'abitazione in cui risiedono, secondo i dati registrati in occasione del censimento ISTAT 2011. Nel comune di Bologna, 57'279 delle 192'573 famiglie totali vivevano in affitto. Si tratta di una quota molto alta (29.74\%), ben superiore alla media regionale e nazionale: a Bologna una famiglia su tre risiedeva in affitto ed è facilmente comprensibile come questo determini un'elevata domanda di locazioni residenziali. II dato risulta ancor più sorprendente alla luce del fatto che non comprende gli studenti fuorisede, ipotizzando che questi non acquisiscano la residenza in città. La popolazione censita, anche detta legale, infatti, è quella residente, come dichiarato nella Gazzetta Ufficiale n.294 del 18 dicembre 2012. Generalmente gli studenti fuorisede non acquisiscono la residenza in città, a maggior ragione nei primi anni, anche per non perdere alcuni vantaggi relativi al contratto di locazione, riservati appunto agli studenti senza residenza in città (questo fenomeno ha però subito una 
brusca inversione di tendenza nell'ultimo anno a causa del passaggio all'utilizzo dell'ISEE per il computo delle tasse universitarie da parte dell'Alma Mater Studiorum).

I dati del Censimento 2011 aiutano a stimare la domanda di abitazioni in affitto; diversamente, per quantificare l'offerta, si fa riferimento alle statistiche catastali pubblicate annualmente da $\mathrm{OMI}^{19}$. Nella Tabella 13 sono riportate le abitazioni di tipo residenziale, ossia quelle classificate nel gruppo A ad esclusione di A/10 (uffici e studi privati), per il Comune di Bologna. II totale di queste ammontava a 222'133, anche se ai fini della nostra analisi abbiamo deciso di considerare esclusivamente le tipologie di abitazione di interesse per i nuclei familiari di riferimento: abitazioni di tipo civile, economico, popolare e ville, per un totale di 219'961 alloggi nel Comune di Bologna.

Tabella 13. Numero di unità immobiliari urbane (U.I.U.) di tipo residenziale. Statistiche catastali 2011.

Numero di unità immobiliari urbane (U.I.U.) di tipo residenziale - Statistiche catastali 2011

\begin{tabular}{|l|c|r|}
\hline A/1 & Abitazioni di tipo signorile & 85 \\
\hline A/2 & Abitazioni di tipo civile & 1750942 \\
\hline A/3 & Abitazioni di tipo economico & 15550817 \\
\hline A 4 & Abitazioni di tipo popolare & 4450741 \\
\hline A/5 & Abitazioni di tipo ultrapopolare & 150413 \\
\hline A 6 & Abitazioni di tipo rurale & 105 \\
\hline A 7 & Abitazioni in villini & 150461 \\
\hline A/8 & Abitazioni in ville & 134 \\
\hline A $/ 9$ & Castelli, palazzi di eminenti pregi artistici o storici & 435 \\
\hline A 11 & Abitazioni ed alloggi tipici dei luoghi & 0 \\
\hline Totale & & 222133 \\
\hline Totale di interesse & A/2 + A/3 + A/4 + A/7 & 219961 \\
\hline
\end{tabular}

Per arrivare ad una determinazione più precisa di domanda e offerta è opportuno tenere in considerazione altri elementi. Delle 57'279 famiglie residenti in affitto secondo il censimento 2011, circa 12'000 risiedono nelle abitazioni messe a disposizioni da ACER. Dal momento che il mercato di riferimento per i contratti di locazione a canone concordato e a libero mercato esula dalle famiglie che cercano risposta nell'edilizia residenziale pubblica, per determinare più correttamente domanda e offerta abbiamo sottratto queste $12^{\prime} 000$ abitazioni, e quindi famiglie, sia dalla domanda potenziale (le 57'279 famiglie) sia dal numero complessivo di abitazioni di interesse presenti nel Comune (219'961). In questo modo si ottiene uno stock complessivo di 207'961 abitazioni e una domanda potenziale di 45'279 famiglie.

\footnotetext{
${ }^{19}$ Si è scelto di utilizzare le statistiche catastali del 2011 per poter confrontare lo stock di alloggi complessivo con i dati effettivi del censimento ISTAT. Link: http://www.agenziaentrate.gov.it/wps/content/nsilib/nsi/schede/fabbricatiterreni/omi/pubblica zioni/statistiche+catastali/archivio+statistiche+catastali
} 
Calcolando nuovamente la percentuale di famiglie in affitto nel Comune di Bologna secondo il censimento 2011, si ottiene un dato pari al 25.08\% (45279/180573). Applicando la suddetta percentuale al numero totale di abitazioni, al netto di quelle ACER, si ottiene un valore pari a 52'147 abitazioni nel 2011.

Di queste, una parte sarà necessariamente destinata agli studenti: nei capitoli successivi si spiegherà come per il proprietario affittare il proprio immobile a studenti possa essere più vantaggioso rispetto a locarlo ad una famiglia. Nel 2011 il polo cittadino dell'Università di Bologna contava 31'474 studenti fuorisede, a fronte di un'offerta di posti letto in studentato pari a $1^{\prime} 414 .{ }^{20}$ Ipotizziamo che $30^{\prime} 060$ fuorisede non riescano ad accedere alle soluzioni offerte da ER.GO. e supponiamo una media di 3 studenti per appartamento (data la dimensione media delle abitazioni disponibili a sul mercato bolognese) tutti locati nel comune di Bologna, di conseguenza saranno necessarie $10^{\prime} 020$ abitazioni per sopperire a tale domanda di alloggio.

L'offerta di abitazioni in affitto si riduce quindi a 42'127 per le famiglie. Questo valore dell'offerta stimata va confrontato con la domanda registrata nel 2011 di 45'279 famiglie. Secondo i valori stimati per il 2011 esisteva un eccesso di domanda di 3'152 famiglie, discrepanza non indifferente. Volendo trasporre ad oggi l'analisi, la situazione risulterebbe maggiormente aggravata dal fatto che 1'966 "case intere" vengono affittate nel mercato turistico a breve termine su Airbnb ${ }^{\text {TM }},{ }^{21}$ riducendo ulteriormente l'offerta del mercato locativo residenziale. Inoltre, oggi l'Università conta circa 35'000 fuori sede con un ulteriore aggravio sul mercato per almeno altre mille e più unità abitative. Queste prime valutazioni, sebbene basate su assunzioni forti, quali una sostanziale inelasticità del mercato dell'offerta, mettono alla luce una potenziale domanda inevasa di 6'000 alloggi in locazione. Queste 6'000 famiglie (o un compositum di studenti e famiglie) si ritrova nella condizione di uscire dai confini comunali ovvero di vivere in abitazioni condivise con altre famiglie. L'eccesso di domanda "stimato" in 6'000 unità non implica che queste persone non abbiano un tetto sulla testa, ma più semplicemente che non trovino sul mercato bolognese una soluzione per loro economicamente sostenibile o qualitativamente adeguata.

\section{Le abitazioni a canone concordato}

Il contratto a canone concordato è una tipologia di contratto locativo previsto dalla legge 431/98. Esso è stato pensato per alcune aree soggette ad alta densità abitativa, nelle quali la domanda di immobili maggiore dell'offerta tende a far lievitare i prezzi di libero mercato, rendendo difficile l'accesso alla casa per le famiglie più fragili. I contratti locativi a canone concordato rappresentano un tentativo di soluzione al

\footnotetext{
${ }^{20}$ I dati relativi agli studenti sono tratti dal data warehouse d'ateneo; per un maggior descrizione si rimanda al capitolo 7, interamente dedicato agli studenti.

${ }^{21}$ Per un maggior dettaglio sulla questione si rimanda al capitolo 8 che affronta in modo dettagliato il mercato locativo a breve termine.
} 
problema abitativo da parte degli enti locali, i quali si interpongono come mediatori tra locatari e locatori, con l'obiettivo di facilitare l'incontro tra le due parti.

Questa tipologia contrattuale è applicabile in presenza di accordi territoriali tra i rappresentanti delle organizzazioni di proprietari e inquilini, accordi che mirano in particolare a definire valori massimi e minimi dei prezzi applicabili. A differenza del canone libero, in cui le parti stabiliscono liberamente il canone e altri aspetti della locazione, quello concordato non può discostarsi dall'accordo territoriale. Questo definisce prezzi annuali di locazione al metro quadro minimi e massimi, creando diversi intervalli di scelta dei prezzi sulla base del numero dei vani, della localizzazione della abitazione sul territorio cittadino e delle caratteristiche dell'alloggio (numero di elementi di pregio). ${ }^{22}$

Per incentivare la conclusione di contratti di locazione, gli enti territoriali possono rinunciare ad alcune entrate per venire incontro alle esigenze delle due parti e proporre ad entrambe alcuni vantaggi. Per gli inquilini il vantaggio è legato al minor prezzo d'affitto rispetto al canone di mercato: si vogliono in questo senso tutelare le famiglie più deboli che potrebbero non permettersi un canone libero con prezzi crescenti. Per garantire un vantaggio al proprietario, che accetta di ricevere un minor incasso rispetto al libero mercato, sono previste una serie di agevolazioni e sconti fiscali. La più importante è sicuramente la riduzione della cedolare secca: $21 \%$ per gli immobili locati a mercato, $19 \%$ fino al 2013 , poi ridotta al $15 \%$ ed infine al $10 \%$ per il quadriennio 2014-2017 23 - per quelli locati a canone concordato. Oltre a questo vantaggio, l'applicazione di questa forma contrattuale garantisce al proprietario uno sconto su altre imposte, ad esempio l'IMU laddove i comuni lo permettano. L'accordo attualmente in vigore per l'area metropolitana di Bologna, che avrà durata triennale, è stato rinnovato il 26 settembre 2017 ed è entrato in vigore il 9 ottobre.

Per analizzare il mercato locativo residenziale bolognese, in questa ricerca ci si avvale delle informazioni su metratura e localizzazione dei canoni concordati stipulati all'interno del comune di Bologna negli anni 2010, 2012 e 2014, sulla base delle informazioni disponibili al Comune stesso in funzione delle richieste di agevolazioni IMU. La base informativa comprende poco più di 25'000 unità per i primi due anni e di circa 30'000 unità per lo stock 2014; tuttavia si è scelto di eliminare dal campione le unità abitative di metratura non idonea alla permanenza stabile e dignitosa di una famiglia. Si sono quindi escluse dall'analisi tutte le abitazioni con metratura al di sotto

\footnotetext{
22 Tra gli elementi di pregio, facendo riferimento all'accordo territoriale della città metropolitana di Bologna in vigore dal 9 ottobre 2017, figurano: tipologia di certificazione catastale $A / 1-A / 2$ $A / 3-A / 7$ - A/8 - A/9; autorimessa singola; posto auto esclusivo; accesso all'immobile fino al secondo piano o, se superiori, con ascensore; presenza di un riscaldamento autonomo o di un sistema di contabilizzazione delle calorie se l'impianto è centralizzato; porta blindata o sistema di allarme o cancello e doppi vetri; condizionamento; area verde condominiale; area verde esclusiva; doppio servizio; interventi di risparmio energetico; cantina o soffitta; cortile con possibilità di parcheggio in area condominiale; balcone o terrazza o lastrico solare di pertinenza esclusiva; anno di costruzione dell'alloggio o di recupero edilizio importante successivo allo 01/01/2000.

${ }^{23}$ La Finanziaria 2018 ha poi esteso l'agevolazione fino al 2019.
} 
dei $30 \mathrm{mq}$. Ne risulta un parco abitativo pari rispettivamente a 20'988, 20'588 e $25^{\prime} 345$. Le informazioni relative al numero totale di osservazioni e a quelle effettivamente utilizzate nell'analisi sono riportate nella Tabella 14.

Considerando che lo stock complessivo di abitazioni è rimasto pressoché stabile rispetto al 2011 e utilizzando il numero di abitazioni locate precedentemente calcolato per il 2011 (43.862), i contratti di locazione a canone concordato rappresentano, nel 2014 , il $69.79 \%$ di tutti i contratti locativi residenziali, lasciando la percentuale residua al libero mercato (valore assolutamente in linea con i risultati dell'indagine campionaria). Analogamente il peso esercitato dai contratti a canone concordato sul totale del mercato locativo residenziale è del $57.07 \%$ sia nel 2010 che nel 2012.

Tabella 14 - Composizione dataset abitazioni a canone concordato.

\begin{tabular}{|c|r|r|r|}
\hline & 2010 & 2012 & 2014 \\
\hline Osservazioni iniziali & 25031 & 25033 & 30612 \\
\hline Osservazioni eliminate & 4043 & 4445 & 5267 \\
\hline \% osservazioni eliminate & 16.15 & 17.76 & 17.21 \\
\hline Osservazioni finali & 20988 & 20588 & 25345 \\
\hline
\end{tabular}

I tre stock di alloggi 2010, 2012 e 2014 costituiscono il riferimento di questa analisi per l'intero periodo considerato (2009-2017). In particolare, le abitazioni registrate nel 2010 fungeranno da popolazione statistica di riferimento per l'arco temporale compreso tra 2009 e 2011, quelle registrate nel 2012 per il 2012 e il 2013, mentre si adotterà lo stock più recente per tutti gli anni successivi.

Tabella 15 - Numero di alloggi locate a canone concordato per anno e relative variazioni.

\begin{tabular}{|c|r|r|r|}
\hline Numero osservazioni & 2010 & 2012 & 2014 \\
\hline $\begin{array}{c}\text { Variazione percentuale numero totale alloggi } \\
\text { rispetto allo stock precedente }\end{array}$ & 20988 & 20588 & 25345 \\
\hline \% alloggi che permangono tra due stock consecutivi & - & -1.91 & 23.09 \\
\hline
\end{tabular}

L'utilizzo dell'effettivo numero di contratti in essere consente una migliore rappresentazione del mercato reale, con riferimento alle locazioni in atto a canone

\footnotetext{
24 Il matching è stato effettuato, dopo aver eliminato le abitazioni con metratura inferiore ai 30mq, confrontando indirizzo, coordinate geografiche e superficie dell'appartamento. È infatti possibile che all'interno di uno stesso edificio (stesso indirizzo e medesime coordinate) siano presenti più abitazioni locate a canone concordato: utilizzare la metratura permette, salvo in rari casi, di tenere in considerazione questo elemento.
} 
concordato. L'analisi temporale dei prezzi al metro quadro non risente infatti dei diversi campioni utilizzati durante il periodo; al contrario, i canoni di locazione mensili saranno influenzati in quanto calcolati a partire dalle superfici delle specifiche abitazioni.

Con riferimento alle abitazioni oggetto dell'indagine, come si può osservare nella Tabella 15, il numero di alloggi locati a canone concordato nel 2012 è inferiore di 400 unità (-1.91\%) rispetto a quelle del 2010. Benché il totale rimanga pressoché invariato, analizzando il tasso di variazione delle singole abitazioni tra i diversi stock annuali, il $76.5 \%{ }^{25}$ delle abitazioni presenti nello stock 2010 continua ad essere allocata a canone concordato anche nel 2012. La percentuale di alloggi non presenti nel dataset 2010, ossia le nuove locazioni rispetto a quelle precedenti, è notevole e giustifica la scelta di adottare i diversi stock disponibili anziché utilizzarne uno unico.

Lo stock 2014 presenta un numero di contratti in essere nettamente maggiore: si passa dalle 20'588 abitazioni del 2012 alle 25'345 del 2014. L'alta percentuale di variazione $(+23.09 \%)$ potrebbe essere dettata dalle modifiche sui prezzi massimi e minimi determinate dall'accordo 2013 e dalla riduzione della cedolare secca applicabile a questa tipologia di contratti. In realtà, poiché l'accordo del 2013 ha ridotto i prezzi di locazione a canone concordato, è più realistico credere che l'aumento dell'offerta, determinata dalla disponibilità dei proprietari, sia dovuto in larga parte all'agevolazione fiscale introdotta piuttosto che alla riduzione dei prezzi. La diminuzione dei prezzi è stata infatti inferiore alla dimensione dell'agevolazione fiscale.

A fronte di un incremento del $23.09 \%$ del numero di alloggi totali, il $93.10 \%$ di quelli presenti nello stock 2012 continuano ad essere locate a canone concordato anche nel 2014. Questi valori mostrano come l'offerta di contratti a canone concordato sia notevolmente aumentata tra il 2012 e il 2014, ma come larga parte dei contratti in essere nel 2012 continui ad avere valore nel 2014. Si tratta di segnali positivi: i contratti in essere a canone concordato continuano a rimanere tali, e il numero complessivo di abitazioni aumenta a fronte di nuove entrate in tale mercato, pur conservando buona parte di quelle precedentemente presenti.

La Tabella 16 presenta il numero di abitazioni locate a canone concordato di cui si compone la nostra banca dati, per gli anni relativi agli stock disponibili e suddiviso nelle aree omogenee OMI.

\footnotetext{
${ }^{25}$ La percentuale è calcolata escludendo ancora una volta le abitazioni con una superficie inferiore ai $30 \mathrm{mq}$ e quelle non identificate unicamente (in alcuni casi abitazioni diverse possono essere identificate dallo stesso indirizzo, essendo ad esempio due interni diversi, di uguale metratura, all'interno di uno stesso condominio).
} 
Tabella 16. Numero di abitazioni locate a canone concordato componenti il dataset e variazioni delle stesse tra i diversi stock utilizzati, suddivise per aree OMI.

\begin{tabular}{|c|c|c|c|c|c|c|c|c|c|c|}
\hline \multicolumn{5}{|c|}{ Numero di abitazioni } & \multicolumn{6}{|c|}{ Variazioni tra stock 26} \\
\hline \multirow{2}{*}{$\begin{array}{l}\text { Aree } \\
\text { OMI }\end{array}$} & \multicolumn{2}{|c|}{2010} & \multicolumn{2}{|c|}{2012} & \multicolumn{2}{|c|}{2014} & \multicolumn{2}{|c|}{$\Delta_{12-10}(\%)$} & \multicolumn{2}{|c|}{$\Delta_{14-12}(\%)$} \\
\hline & $\mathrm{N}$ & $\%$ & $\mathrm{~N}$ & $\%$ & $\mathrm{~N}$ & $\%$ & Zona & Assoluta & Zona & Assoluta \\
\hline B1 & 706 & 3.36 & 747 & 3.63 & 905 & 3.57 & 5.81 & 10.25 & 21.15 & 3.32 \\
\hline B7 & 70 & 0.33 & 78 & 0.38 & 92 & 0.36 & 11.43 & 2.00 & 17.95 & 0.29 \\
\hline C10 & 124 & 0.59 & 126 & 0.61 & 155 & 0.61 & 1.61 & 0.50 & 23.02 & 0.61 \\
\hline C11 & 673 & 3.21 & 682 & 3.31 & 847 & 3.34 & 1.34 & 2.25 & 24.19 & 3.47 \\
\hline C3 & 440 & 2.1 & 405 & 1.97 & 494 & 1.95 & -7.95 & -8.75 & 21.98 & 1.87 \\
\hline C4 & 203 & 0.97 & 211 & 1.02 & 249 & 0.98 & 3.94 & 2.00 & 18.01 & 0.80 \\
\hline C5 & 410 & 1.95 & 407 & 1.98 & 473 & 1.87 & -0.73 & -0.75 & 16.22 & 1.39 \\
\hline C6 & 572 & 2.73 & 576 & 2.8 & 671 & 2.65 & 0.7 & 1.00 & 16.49 & 2.00 \\
\hline C7 & 84 & 0.4 & 90 & 0.44 & 119 & 0.47 & 7.14 & 1.50 & 32.22 & 0.61 \\
\hline C8 & 460 & 2.19 & 470 & 2.28 & 536 & 2.12 & 2.17 & 2.50 & 14.04 & 1.39 \\
\hline C9 & 339 & 1.62 & 347 & 1.69 & 420 & 1.66 & 2.36 & 2.00 & 21.04 & 1.54 \\
\hline D16 & 629 & 3 & 654 & 3.18 & 778 & 3.07 & 3.97 & 6.25 & 18.96 & 2.61 \\
\hline D17 & 613 & 2.92 & 599 & 2.91 & 722 & 2.85 & -2.28 & -3.50 & 20.53 & 2.59 \\
\hline D19 & 623 & 2.97 & 595 & 2.89 & 721 & 2.85 & -4.49 & -7.00 & 21.18 & 2.65 \\
\hline D2 & 1107 & 5.27 & 1064 & 5.17 & 1302 & 5.14 & -3.88 & -10.75 & 22.37 & 5.01 \\
\hline D20 & 1791 & 8.53 & 1707 & 8.29 & 2132 & 8.41 & -4.69 & -21.00 & 24.90 & 8.94 \\
\hline D21 & 833 & 3.97 & 812 & 3.94 & 1004 & 3.96 & -2.52 & -5.25 & 23.65 & 4.04 \\
\hline D22 & 680 & 3.24 & 698 & 3.39 & 860 & 3.39 & 2.65 & 4.50 & 23.21 & 3.41 \\
\hline D23 & 244 & 1.16 & 252 & 1.22 & 289 & 1.14 & 3.28 & 2.00 & 14.68 & 0.78 \\
\hline D24 & 1053 & 5.02 & 963 & 4.68 & 1228 & 4.85 & -8.55 & -22.50 & 27.52 & 5.57 \\
\hline D25 & 1165 & 5.55 & 1154 & 5.61 & 1403 & 5.54 & -0.94 & -2.75 & 21.58 & 5.24 \\
\hline D3 & 890 & 4.24 & 880 & 4.27 & 1128 & 4.45 & -1.12 & -2.50 & 28.18 & 5.22 \\
\hline D4 & 2035 & 9.7 & 1953 & 9.49 & 2470 & 9.75 & -4.03 & -20.50 & 26.47 & 10.88 \\
\hline D5 & 1587 & 7.56 & 1563 & 7.59 & 1915 & 7.56 & -1.51 & -6.00 & 22.52 & 7.40 \\
\hline D6 & 0 & 0 & 0 & 0 & 0 & 0 & - & 0 & - & 0 \\
\hline D7 & 535 & 2.55 & 486 & 2.36 & 610 & 2.41 & -9.16 & -12.25 & 25.51 & 2.61 \\
\hline D8 & 1417 & 6.75 & 1352 & 6.57 & 1643 & 6.48 & -4.59 & -16.25 & 21.52 & 6.12 \\
\hline D9 & 384 & 1.83 & 356 & 1.73 & 474 & 1.87 & -7.29 & -7.00 & 33.15 & 2.48 \\
\hline E1 & 225 & 1.07 & 243 & 1.18 & 277 & 1.09 & 8 & 4.50 & 13.99 & 0.72 \\
\hline R1 & 987 & 4.7 & 1009 & 4.9 & 1291 & 5.09 & 2.23 & 5.50 & 27.95 & 5.93 \\
\hline R2 & 25 & 0.12 & 25 & 0.12 & 21 & 0.08 & - & 0.00 & -16 & -0.08 \\
\hline R3 & 48 & 0.23 & 53 & 0.26 & 64 & 0.25 & 10.42 & 1.25 & 20.75 & 0.23 \\
\hline R4 & 36 & 0.17 & 31 & 0.15 & 49 & 0.19 & -13.9 & -1.25 & 58.06 & 0.38 \\
\hline Tot. & 20988 & 100 & 20588 & 100 & 25342 & 100 & -1.91 & - & 23.09 & - \\
\hline
\end{tabular}

${ }^{26}$ Le variazioni di zona rappresentano le percentuali di variazione relative alla singola zona OMI (numero della variazione di zona rapportato al numero di abitazioni totali della specifica zona presente nel dataset più datato); le variazioni assolute sono calcolate in funzione della variazione registrata per tutto il campione (numero della variazione di zona rapportata al numero assoluto della variazione su tutto il campione). 
Le variazioni percentuali 2010-2012 del numero di abitazioni suddivise per zone OMI sono molto eterogenee: per alcune di queste zone il numero di alloggi rimane costante, mentre altre mostrano variazioni superiori al $10 \%$ in valore assoluto. Nell'analisi di tali variazioni, però, è importante considerare anche il peso delle stesse sul totale del numero di alloggi cambiati tra i due stock (colonna "Assoluta"). Ad esempio, la variazione 2010-12 più alta in valore assoluto è il $-13.89 \%$ registrato per la zona R4; si tratta però di una differenza di 5 abitazioni, il cui peso complessivo sul numero di alloggi variate tra i due stock è dell'1.25\%. In questo senso assumono rilevanza le variazioni registrate nelle zone D20 "Saffi-Santa Viola-Barca" (-4.69\%), D24 “Murri" (-8.55\%) e D4 "Mazzini-Fossolo" (-4.03\%), che hanno pesi maggiori del $20 \%$ rispetto al numero totale di variazioni tra i due stock.

La Tabella 17, invece, ripartisce le abitazioni presenti nel nostro database per area di applicazione del canone concordato, facendo sempre riferimento ai tre anni considerati.

Relativamente ad esse le variazioni sono più contenute rispetto a quelle delle zone OMI: nel 2012 lo stock di alloggi della zona di pregio è il 3.59\% superiore allo stesso stock 2010; diversamente le zone A e B vedono ridurre il rispettivo numero di abitazioni nel secondo anno rilevato. Per quanto queste variazioni siano minime, risulta interessante notare come nel 2012, rispetto al 2010, sia aumentato il numero di contratti stipulati nella zona più costosa di Bologna (zona di pregio). Nonostante questo aumento, osservando la distribuzione all'interno dello stesso anno, questa zona rimane quella con la concentrazione più bassa di abitazioni (15.15\% dello stock 2012) tra tutte quelle allocate a canone concordato.

I tassi di variazione nel numero di abitazioni presenti nelle zone tra 2012 e 2014 sono sicuramente influenzati dall'aumento del $23.09 \%$ del numero totale di alloggi componenti il dataset. Di conseguenza, le percentuali di variazione all'interno delle singole zone OMI registrano valori molto elevati. Anche in questo caso, però, è doveroso considerare il peso delle variazioni interne alle zone rispetto alla variazione totale tra i due stock. In questo senso la riduzione del $16 \%$, unico valore negativo, relativo alla zona R2 "Agricola Nord-Ovest", ha un peso marginale (0.08\%) sul campione complessivo.

Tabella 17 - Numero di abitazioni locate a canone concordato componenti il dataset, suddivise per zone di applicazione del canone concordato.

\begin{tabular}{|c|c|c|c|c|c|c|c|c|}
\hline & \multicolumn{8}{|c|}{ Numero di abitazioni } \\
\hline \multirow{2}{*}{$\begin{array}{c}\text { Zona Canone } \\
\text { Concordato }\end{array}$} & \multicolumn{2}{|c|}{2010} & \multicolumn{2}{|c|}{2012} & \multirow{2}{*}{$\begin{array}{r}\Delta_{12-10} \\
\%\end{array}$} & \multicolumn{2}{|c|}{2014} & \multirow{2}{*}{$\begin{array}{r}\Delta_{14-12} \\
\%\end{array}$} \\
\hline & $\mathrm{N}$ & $\%$ & $\mathrm{~N}$ & $\%$ & & $\mathrm{~N}$ & $\%$ & \\
\hline Zona di Pregio & 3012 & 14.35 & 3120 & 15.15 & 3.59 & 3734 & 14.73 & 19.68 \\
\hline Zona A & 8031 & 38.26 & 7838 & 38.07 & -2.40 & 9574 & 37.77 & 22.15 \\
\hline Zona B & 9945 & 47.38 & 9630 & 46.77 & -3.17 & 12037 & 47.49 & 25.00 \\
\hline Totale & 20988 & 100 & 20588 & 100 & -1.91 & 25345 & 100 & 23.09 \\
\hline
\end{tabular}


Più semplice è l'analisi delle variazioni percentuali relative alla suddivisione in zone di applicazione del canone concordato: I'aumento complessivo del $23.09 \%$ può essere scomposto in un aumento del $19.68 \%$ delle abitazioni localizzate nella zona di pregio, del $22.15 \%$ nella zona A e del $25 \%$ nella zona B. È quindi quest'ultima zona, quella con la maggior concentrazione di abitazioni (47.49\% delle abitazioni totali nel 2014 ) che vede aumentare maggiormente il numero di contratti a canone concordato. Va però osservato come la zona $\mathrm{B}$ sia la più ampia e sarebbe opportuno considerare indicatori di densità che tengano conto non solamente del numero di abitazioni, ma anche della superficie territoriale della zona.

La Tabella 18 riporta per ogni zona OMI, oltre alla relativa superficie territoriale espressa in chilometri quadrati, la densità ${ }^{27}$ di alloggi locati a canone concordato per ognuno degli anni relativi agli stock disponibili e le relative variazioni percentuali delle stesse.

La Tabella 18 conferma quanto precedentemente illustrato: esiste un'importante eterogeneità nella localizzazione delle abitazioni affittate a canone concordato tra le diverse zone OMI. La densità combina il numero delle stesse con la superficie del territorio di riferimento, indicando il numero di abitazioni per chilometro quadrato.

Il complessivo aumento del numero totale di abitazioni locate a canone concordato tra il 2012 e il 2014 risulta in una maggiore densità registrata nel 2014 di questa tipologia di abitazioni, come dimostrano i valori medi: 644.61 alloggi/km2 nel 2012 e 758.03 alloggi/km2 nel 2014. Tale aumento della densità si registra per tutte le zone OMI di Bologna, con I'unica eccezione della zona R2 (Zona rurale - Agricola Nord) che mostra una riduzione del 16\% tra 2012 e 2014 (anche se, in termini assoluti, si tratta di una riduzione di 4 abitazioni: 25 nel 2012 e 21 nel 2014).

A fronte di zone OMI con una densità molto bassa (la zona meridionale R4 - area collinare - registra nel 2010 solamente 1.30 abitazioni locate a canone concordato per chilometro quadrato), esistono zone $\mathrm{OMI}$ in cui la concentrazione è molto elevata. La grande eterogeneità è evidenziata dalle statistiche di dispersione rispetto alla media: nel 2010 si registra una varianza di 595.07 alloggi/km2, e di 711.23 alloggi $/ \mathrm{km} 2$ nel 2014.

Le variazioni percentuali tra le densità registrate nei diversi anni, ossia dei diversi stock a disposizione, dimostrano l'evoluzione interna alle singole zone del mercato locativo a canone concordato. Tra il 2010 e il 2012, ad esempio, la zona B7 (Zamboni-San Vitale) registra un tasso di variazione dell' $11 \%$ nella densità di abitazioni a canone concordato. All'estremo opposto, la zona R4 registra una riduzione del 14\%. Questi tassi di variazione sono analoghi a quelli precedentemente presentati nella Tabella 16, in quanto la superficie delle zone è sempre costante durante gli anni, e di conseguenza si osservano nuovamente alti tassi di variazione tra 2012 e 2014.

\footnotetext{
${ }^{27}$ La densità è definita rapportando il numero di alloggi e la superficie in chilometri quadrati della zona $\mathrm{OMI}$ in questione.
} 
Tabella 18 - Densità alloggi a canone concordato per zona OMI e variazioni percentuali; media $(\mu)$ e deviazione standard $(\sigma)$.

\begin{tabular}{|c|c|c|c|c|c|c|}
\hline \multirow{2}{*}{ Zona } & \multirow[b]{2}{*}{$\begin{array}{l}\text { Superficie } \\
(\mathrm{km} 2)\end{array}$} & \multirow{2}{*}{$\begin{array}{l}2010 \\
\text { densità } \\
\text { (alloggi/km2) }\end{array}$} & \multirow{2}{*}{$\begin{array}{l}2012 \\
\text { densità } \\
\text { (alloggi/km2) }\end{array}$} & \multirow{2}{*}{$\begin{array}{l}2014 \\
\text { densità } \\
\text { (allogi/km2) }\end{array}$} & \multicolumn{2}{|c|}{$\Delta$ percentuali } \\
\hline & & & & & $\Delta_{2010-12}$ & $\Delta_{2012-14}$ \\
\hline B1 & 0.82 & 860.33 & 910.29 & 1102.83 & 5.81 & 21.15 \\
\hline B7 & 0.10 & 726.48 & 809.51 & 954.80 & 11.43 & 17.95 \\
\hline C10 & 0.11 & 1174.23 & 1193.17 & 1467.79 & 1.61 & 23.02 \\
\hline C11 & 0.74 & 913.93 & 926.16 & 1150.23 & 1.34 & 24.19 \\
\hline C3 & 0.45 & 984.57 & 906.25 & 1105.40 & -7.95 & 21.98 \\
\hline C4 & 0.25 & 806.17 & 837.94 & 988.85 & 3.94 & 18.01 \\
\hline C5 & 0.40 & 1013.55 & 1006.13 & 1169.29 & -0.73 & 16.22 \\
\hline C6 & 0.58 & 992.05 & 998.99 & 1163.75 & 0.70 & 16.49 \\
\hline C7 & 0.07 & 1236.39 & 1324.70 & 1751.55 & 7.14 & 32.22 \\
\hline C8 & 0.54 & 851.15 & 869.66 & 991.78 & 2.17 & 14.04 \\
\hline C9 & 0.24 & 1400.39 & 1433.43 & 1734.99 & 2.36 & 21.04 \\
\hline D16 & 2.17 & 289.26 & 300.76 & 357.79 & 3.97 & 18.96 \\
\hline D17 & 8.42 & 72.78 & 71.12 & 85.72 & -2.28 & 20.53 \\
\hline D19 & 0.21 & 2936.01 & 2804.06 & 3397.86 & -4.49 & 21.18 \\
\hline D2 & 1.13 & 976.22 & 938.30 & 1148.18 & -3.88 & 22.37 \\
\hline D20 & 5.43 & 329.78 & 314.32 & 392.57 & -4.69 & 24.90 \\
\hline D21 & 0.81 & 1026.92 & 1001.03 & 1237.73 & -2.52 & 23.65 \\
\hline D22 & 5.14 & 132.26 & 135.76 & 167.27 & 2.65 & 23.21 \\
\hline D23 & 0.47 & 516.17 & 533.09 & 611.36 & 3.28 & 14.68 \\
\hline D24 & 1.07 & 985.83 & 901.57 & 1149.67 & -8.55 & 27.52 \\
\hline D25 & 2.37 & 491.91 & 487.26 & 592.40 & -0.94 & 21.58 \\
\hline D3 & 3.58 & 248.79 & 246.00 & 315.32 & -1.12 & 28.18 \\
\hline D4 & 6.19 & 328.79 & 315.54 & 399.07 & -4.03 & 26.47 \\
\hline D5 & 3.86 & 411.31 & 405.09 & 496.32 & -1.51 & 22.52 \\
\hline D7 & 1.02 & 523.47 & 475.52 & 596.85 & -9.16 & 25.51 \\
\hline D8 & 3.83 & 369.57 & 352.61 & 428.51 & -4.59 & 21.52 \\
\hline D9 & 6.08 & 63.11 & 58.51 & 77.91 & -7.29 & 33.15 \\
\hline E1 & 11.95 & 18.83 & 20.34 & 23.19 & 8.00 & 13.99 \\
\hline R1 & 25.93 & 38.06 & 38.91 & 49.78 & 2.23 & 27.95 \\
\hline R2 & 4.32 & 5.79 & 5.79 & 4.86 & 0.00 & -16.00 \\
\hline R3 & 11.55 & 4.16 & 4.59 & 5.54 & 10.42 & 20.75 \\
\hline R4 & 27.68 & 1.30 & 1.12 & 1.77 & -13.89 & 58.06 \\
\hline $\boldsymbol{\mu}$ & 4.30 & 647.80 & 644.61 & 785.03 & - & - \\
\hline $\boldsymbol{\sigma}$ & 6.73 & 595.07 & 583.11 & 711.23 & - & - \\
\hline
\end{tabular}

Le mappe sottostanti (Figura 20, Figura 21 e Figura 22) mostrano, per tutti gli anni degli stock di riferimento, le diverse densità di tali alloggi tra le zone OMI, opportunamente suddivise per quintili, riassumendo le informazioni contenute nella Tabella 18. 
Figura 20. Densità di abitazioni a canone concordato per zona OMI - 2010 (quintili).

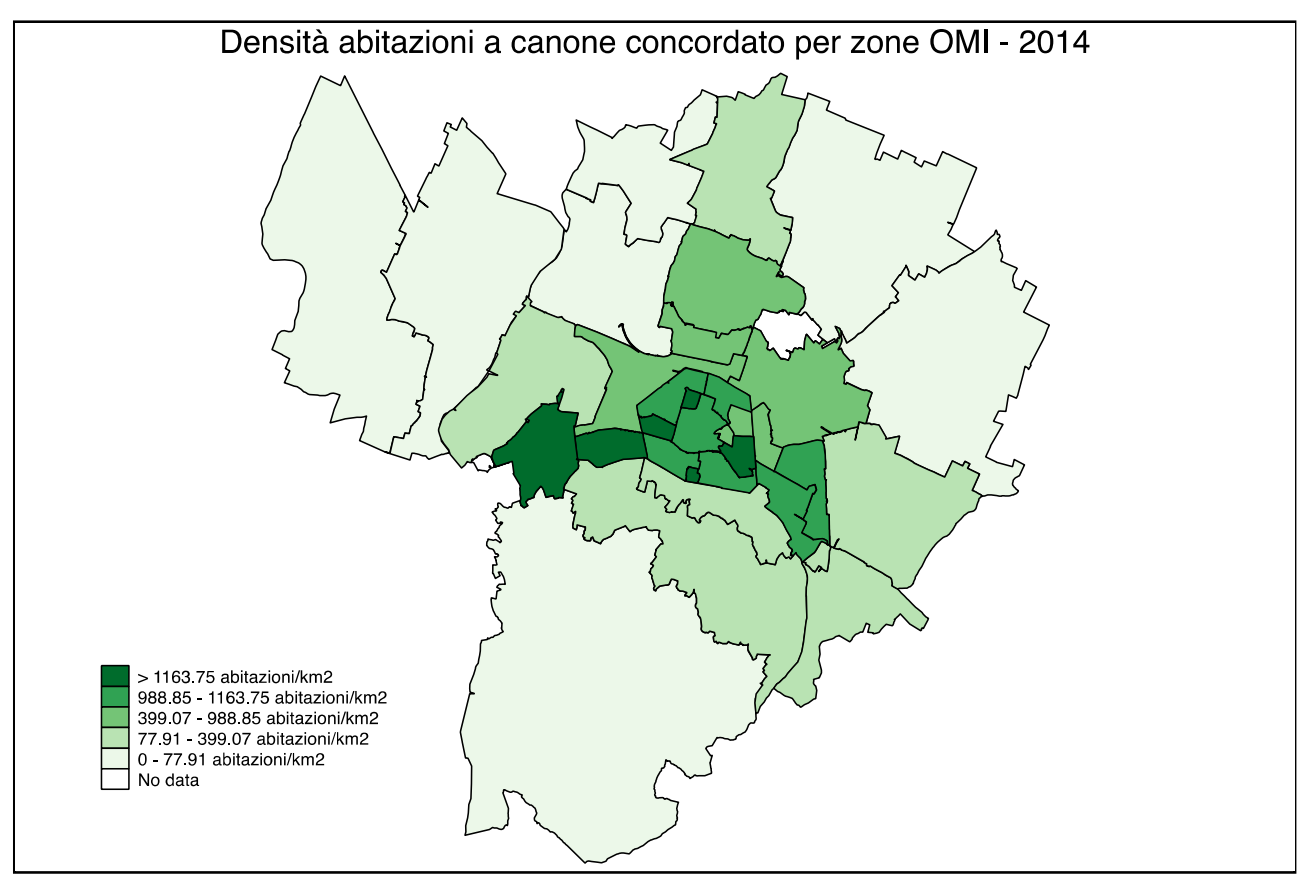

Figura 21. Densità di abitazioni a canone concordato per zona OMI - 2012 (quintili).

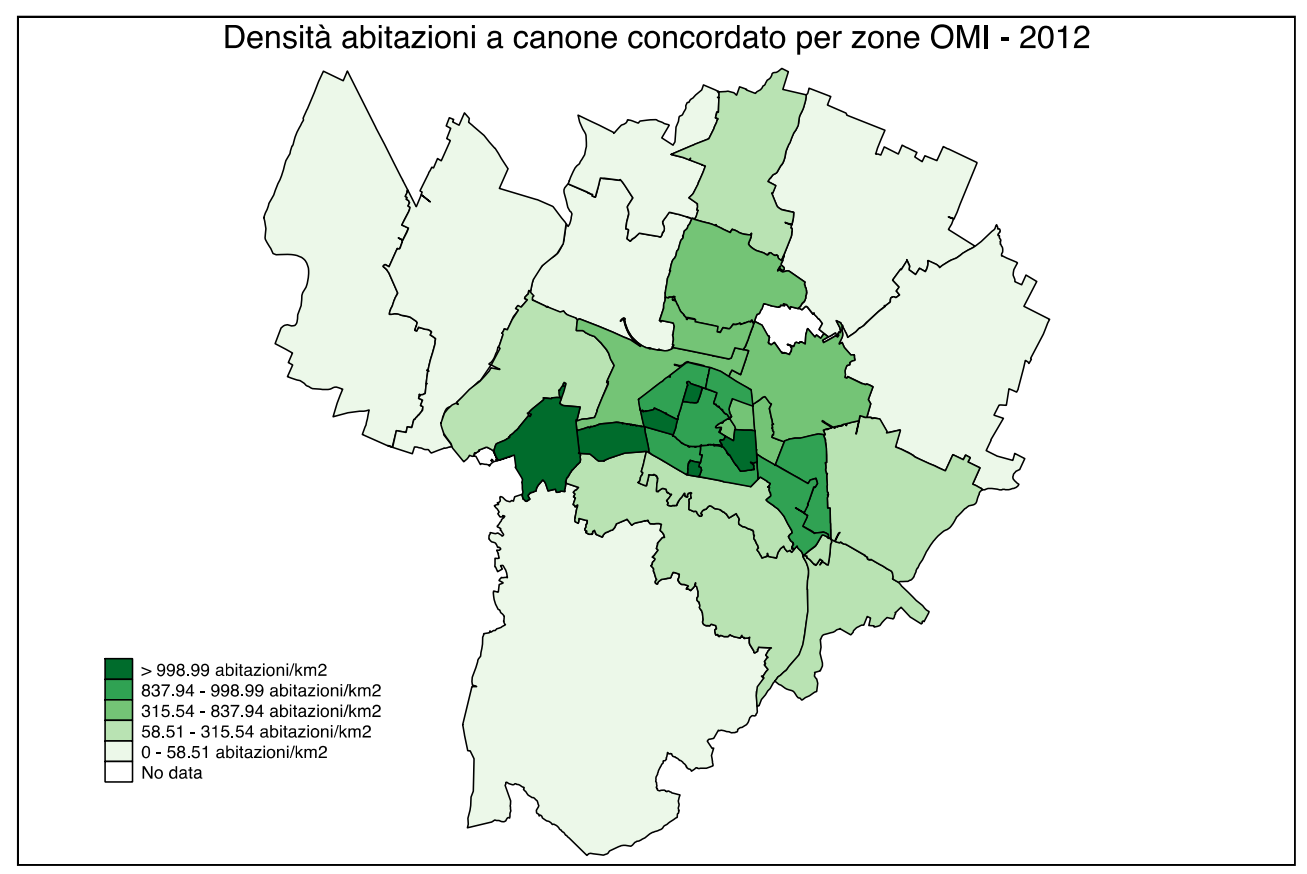


Figura 22. Densità di abitazioni a canone concordato per zona OMI - 2014 (quintili).

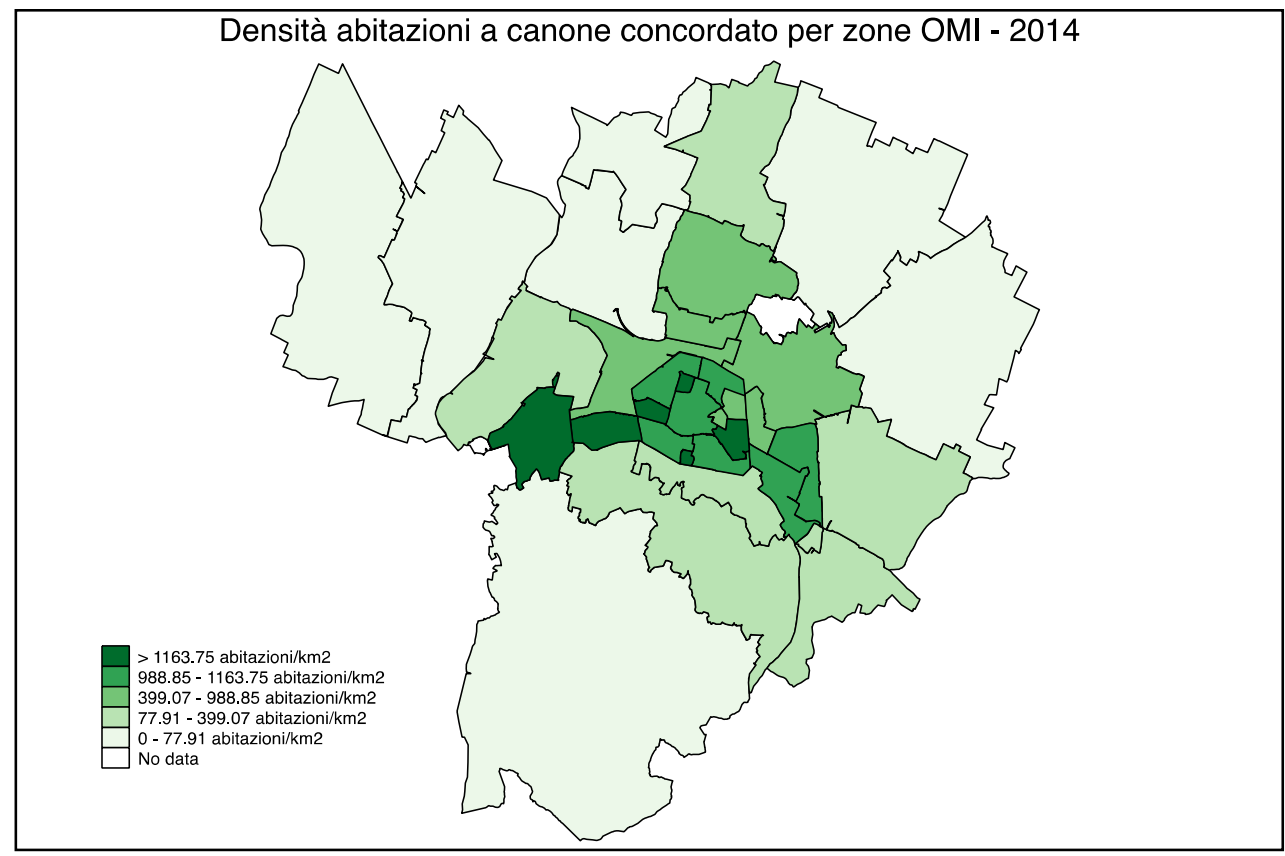

Dalle mappe precedenti (Figura 20, Figura 21 e Figura 22) è possibile osservare la distribuzione e la concentrazione sul territorio delle abitazioni a canone concordato, notando come essa sia particolarmente bassa in varie zone. Per alcune di esse (specie le aree rurali) la causa può essere individuata nella scarsa densità abitativa complessiva, ipotizzando però possa talvolta intervenire una valutazione di convenienza del contratto a canone di mercato, rispetto a quello a canone concordato, nella specifica zona.

Da tutte e tre le figure, con riferimento alla distribuzione per quintili delle densità, risulta evidente come la maggior concentrazione di alloggi locati a canone concordato, rapportate alla superficie della relativa Zona OMI, venga registrata nella fascia territoriale centrale del comune di Bologna, in corrispondenza del centro storico e della cinta appena fuori le mura (escludendo la parte meridionale). È interessante notare come, nonostante cambi lo stock applicato, la distribuzione dei quintili di densità non cambi nei tre anni considerati, e le tre mappe presentino sempre la stessa gradazione di colore per ogni zona. ${ }^{28}$

Le zone D19 (Stadio-Funivia) e D21 (Andrea Costa-Saragozza), insieme ad alcune zone del centro storico, sono quelle con la maggior concentrazione. In queste zone, appartenenti al quintile più alto, la densità media è di circa 1800 alloggi/km2 (stock 2014), con un valore estremamente alto (3'397.86 alloggi/km2 - dato 2014) per la zona D19. Le restanti zone del centro storico (ad esclusione della C4 - Irnerio-Porta San Donato-Petroni), insieme alle zone D2 (San Vitale) e D24 (Murri) costituiscono il nucleo di zone appartenenti al quarto

\footnotetext{
${ }^{28}$ Le statistiche descrittive sono però diverse, in particolare tra 2012 e 2014, per i diversi stock
} 
quintile, con una densità media (2014) leggermente superiore alle 1100 unità per chilometro quadrato. Le zone della prima periferia Nord costituiscono la maggior parte di quelle appartenenti al terzo quintile.

Le zone con la minor densità di abitazioni a canone concordato, appartenenti al primo quintile, sono quelle più periferiche a Nord (con l'esclusione della zona D22 - Corticella) e l'ampia zona collinare a Sud. Il valore medio della densità del primo quantile è di 30.40 alloggi/km2, con riferimento allo stock di alloggi 2014.

I vani

Dopo aver presentato lo stock complessivo utilizzato nel campione, è utile quantificare l'offerta di abitazioni a canone concordato distinguendole per numero di vani. Nel dataset a disposizione sono presenti le superfici delle abitazioni che vengono utilizzate, secondo alcune ipotesi dettagliatamente espresse nella nota metodologica, per determinare il numero di locali di ogni alloggio.

Nel rapporto vengono adottate due diverse definizioni di vani: relativamente all'evoluzione dei prezzi al metro quadro e dei canoni d'affitto mensili viene adottata una definizione estensiva degli stessi; diversamente, per definire l'effettiva quantità di abitazioni offerte sul mercato si è scelto di utilizzare una definizione mutuamente esclusiva del numero di locali.

In accordo con la seconda di queste definizioni, la relazione tra superficie e numero di vani è stata stabilita con riferimento ai dati rilevati in occasione del Censimento ISTAT 2011 sulla distribuzione di frequenza per numero di vani e superficie delle abitazioni bolognesi. I vani delle abitazioni vengono fatti coincidere con la classe di superficie mediana della relativa distribuzione, includendo le classi estreme nella tipologia più vicina ${ }^{29}$. La Tabella 19 sintetizza la definizione adottata in merito all'argomento in questa analisi.

Tabella 19. Definizione mutuamente esclusiva del numero di vani in relazione alla superficie.

\begin{tabular}{|l|l|l|l|l|l|l|}
\hline Numero di vani & 1 & 2 & 3 & 4 & 5 & 6 \\
\hline Classe di superficie (mq) & $30-49$ & $50-59$ & $60-79$ & $80-99$ & $100-119$ & $120+$ \\
\hline
\end{tabular}

La Figura 23 illustra la distribuzione delle diverse tipologie abitative (definite in funzione del numero di vani) negli stock utilizzati interamente considerati (senza suddividere per zone). Tra i tre stock utilizzati non si notano differenze degne di nota. I trilocali rappresentano il tipo di abitazione più diffuso, rappresentando circa un terzo di tutte le abitazioni locate a canone concordato nel Comune di Bologna.

29 Per un maggior dettaglio sulla definizione del numero di vani e per consultare i dati del Censimento ISTAT 2011 si rimanda alla nota metodologica. 
Figura 23. Distribuzione delle abitazioni disponibili per numero di vani - Comune di Bologna.

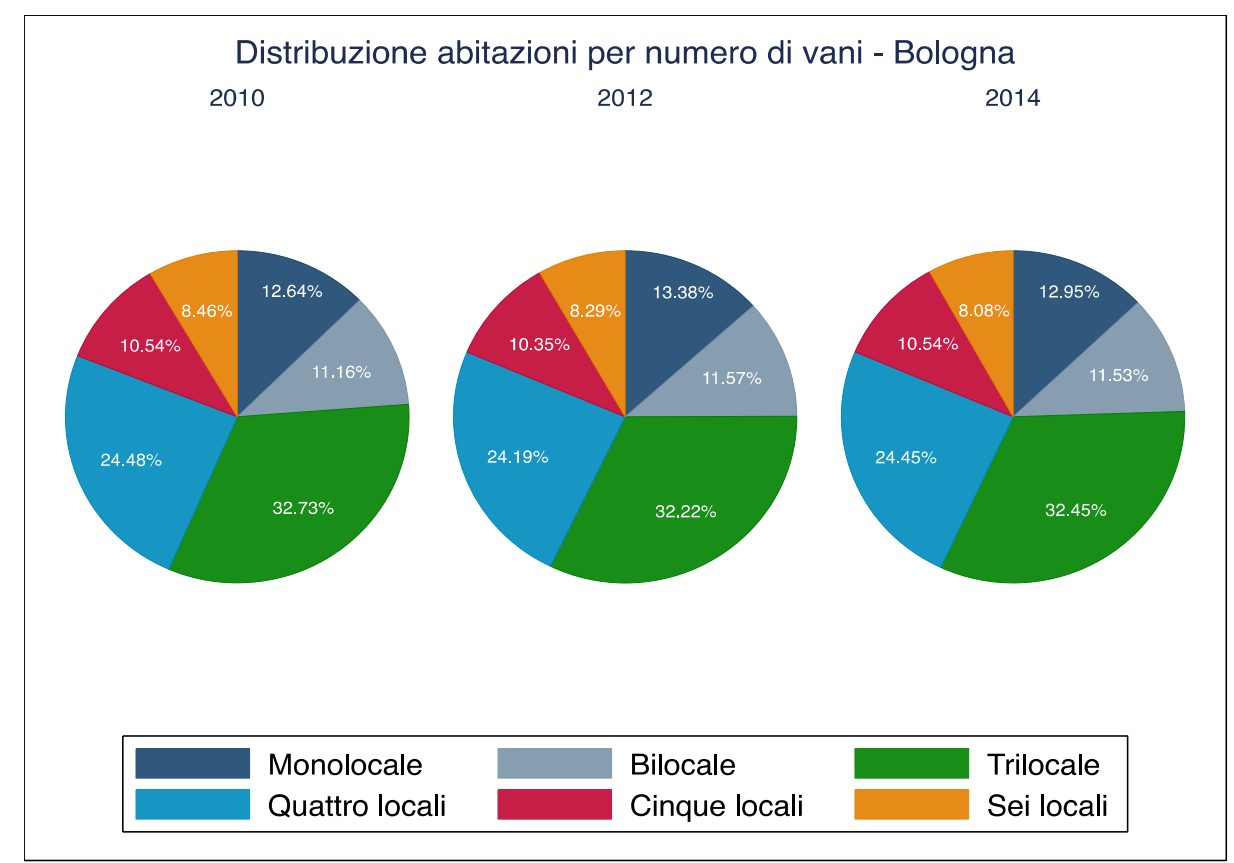

Tabella 20. Numero di abitazioni locate a canone concordato per numero di vani-comune di Bologna.

\begin{tabular}{|c|c|c|c|c|c|c|}
\hline \multicolumn{7}{|c|}{ Offerta di abitazioni a canone concordato } \\
\hline \multirow[t]{2}{*}{ Vani } & \multicolumn{2}{|l|}{2010} & \multicolumn{2}{|l|}{2012} & \multicolumn{2}{|l|}{2014} \\
\hline & $\mathrm{N}$ & $\%$ & $\mathrm{~N}$ & $\%$ & $\mathrm{~N}$ & $\%$ \\
\hline 1 & 2637 & 12.64 & 2736 & 13.38 & 3262 & 12.95 \\
\hline 2 & 2330 & 11.16 & 2366 & 11.57 & 2903 & 11.53 \\
\hline 3 & 6831 & 32.73 & 6589 & 32.22 & 8172 & 32.45 \\
\hline 4 & 5108 & 24.48 & 4948 & 24.19 & 6158 & 24.45 \\
\hline 5 & 2199 & 10.54 & 2116 & 10.35 & 2654 & 10.54 \\
\hline 6 & 1765 & 8.46 & 1696 & 8.29 & 2035 & 8.08 \\
\hline Tot & 20870 & & 20451 & & 25184 & \\
\hline
\end{tabular}

La Tabella 20 riassume i valori presentati nella figura precedente: si tratta dell'offerta di abitazioni, suddivise per tipologia, locate a canone concordato nel Comune di Bologna. Sostanzioso appare essere anche il numero di quadrilocali e la dimensione media ( 3 o 4 vani) copre per ogni anno più della metà delle abitazioni disponibili (circa $57 \%$ in ogni stock disponibile). Le abitazioni piccole (1 o 2 vani) sono leggermente più frequenti di quelle grandi (5 o 6 vani): $24.48 \%$ contro $18.62 \%$ nel 2014 .

Quante persone tale offerta abitativa possa contenere è altresì di interesse per questa analisi. Chiaramente questa stima dipende dalla tipologia di inquilino: si provvede quindi a calcolare tali valori relativamente agli studenti, alle coppie (numero di componenti medio per le famiglie bolognesi), e alle famiglie che a livello teorico sono 
più adatte alla tipologia di abitazione. Nel primo caso supponiamo di poter assegnare uno studente ad ogni vano, per le coppie avremo due persone per ogni tipologia di appartamento, e nell'ultimo caso adottiamo un criterio che sarà successivamente utilizzato per definire la sostenibilità del canone concordato mensile. Nello specifico, il numero ideale di persone per abitazione sia una persona o coppia per i monolocali e bilocali; coppia o nucleo di tre persone per le abitazioni medie (3 e 4 locali) e nuclei di 3 o 4 persone per i 5 e 6 locali. Per calcolare l'offerta abitativa in termini di numero di persone allocabili, consideriamo il nucleo più numeroso tra i due per ogni tipologia abitativa ${ }^{30}$. In questo modo si allocano due persone nei mono e bilocali, 3 persone nei tri e quadrilocali e 4 nelle abitazioni più grandi. ${ }^{31}$

Tabella 21. Numero di persone - studenti, coppie e famiglie - allocabili secondo l'offerta di abitazioni a canone concordato, suddivise per vani.

\begin{tabular}{|l|r|r|r|r|r|r|r|r|r|}
\hline \multicolumn{7}{|c|}{ Numero di persone allocabili secondo l'offerta di canone concordato } \\
\hline \multirow{2}{*}{ Vani } & \multicolumn{3}{|c|}{$\mathbf{2 0 1 0}$} & \multicolumn{3}{|c|}{2012} & \multicolumn{2}{|c|}{2014} \\
\cline { 2 - 12 } & Studenti & Coppie & Famiglie & Studenti & Coppie & Famiglie & Studenti & Coppie & Famiglie \\
\hline $\mathbf{1}$ & 2637 & 5274 & - & 2736 & 5472 & - & 3262 & 6524 & - \\
\hline $\mathbf{2}$ & 4660 & 4660 & - & 4732 & 4732 & - & 5806 & 5806 & - \\
\hline $\mathbf{3}$ & 20493 & 13662 & 20493 & 19767 & 13178 & 19767 & 24516 & 16344 & 24516 \\
\hline $\mathbf{4}$ & 20432 & 10216 & 15324 & 19792 & 9896 & 14844 & 24632 & 12316 & 18474 \\
\hline $\mathbf{5}$ & 10995 & 4398 & 8796 & 10580 & 4232 & 8464 & 13270 & 5308 & 10616 \\
\hline $\mathbf{6}$ & 10590 & 3530 & 7060 & 10176 & 3392 & 6784 & 12210 & 4070 & 8140 \\
\hline Tot & 69807 & 41740 & 51673 & 67783 & 40902 & 49859 & 83696 & 50368 & 61746 \\
\hline
\end{tabular}

Complessivamente, secondo i criteri adottati per definire il numero di persone allocabili nelle abitazioni a disposizione, il numero di studenti che potrebbero trovare spazio negli alloggi locati a canone concordato nel 2014 sono 83'696. II numero è più basso per le famiglie (61'746 persone al massimo) e ancora di più per le coppie (50'368). Negli anni precedenti lo stock è nettamente inferiore alle 25'184 del $2014 \mathrm{e}$, di conseguenza, anche le persone allocabili sono meno. Relativamente alle singole tipologie di abitazioni, quella più frequente (trilocali) offre disponibilità per il maggior numero di persone, seguita dai quadrilocali che, in alcuni casi (studenti 2012 e 2014), riescono addirittura a superare il numero di persone calcolato per i trilocali.

\footnotetext{
${ }^{30}$ La ragione principale per cui si è scelto di considerare il numero di componenti massimo tra i due è da ricercare nella definizione della sostenibilità del canone d'affitto. Nei capitolo seguenti l'analisi sarà sviluppata in questi termini e, volendo essere coerenti, si è qui mantenuto lo stesso criterio. ${ }^{31}$ Questa allocazione è ovviamente il risultato delle ipotesi fatte, ma permette quantomeno di dare una indicazione di massima sul numero di persone che l'attuale offerta locativa a Bologna può soddisfare.
} 
Figura 24. Distribuzione delle abitazioni disponibili per numero di vani - Zona di pregio.

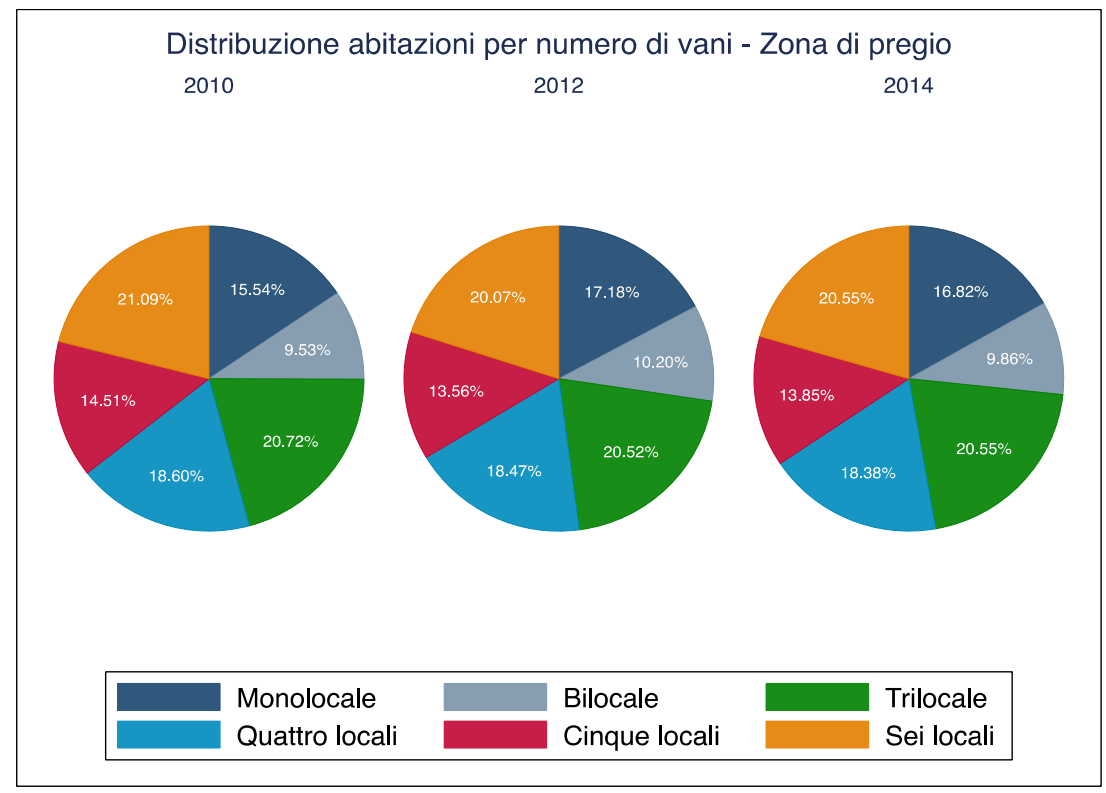

Figura 25. Distribuzione delle abitazioni disponibili per numero di vani - Zona A.

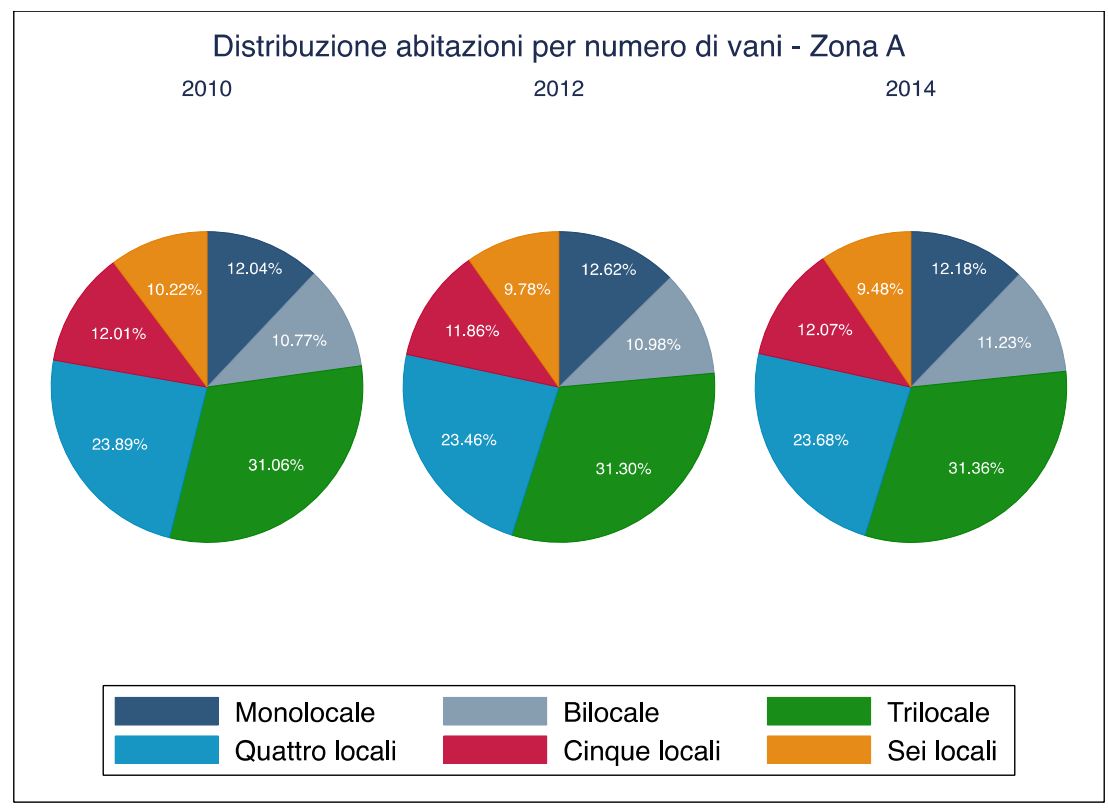

Le Figura 24, Figura 25 e Figura 26 rappresentano la distribuzione delle abitazioni offerte sul mercato a canone concordato suddivise per numero di vani secondo le zone di applicazione del canone concordato. Anche in questo caso, all'interno della stessa zona, le differenze tra gli stock 2010, 2012 e 2014 sono minime. Alcune differenze si riscontrano confrontando tra loro le diverse aree di suddivisione della città: nella zona di pregio le percentuali relative alle abitazioni molto piccole (monolocali) e molto grandi (sei locali) sono nettamente superiori rispetto alle altre zone. Nella zona $\mathrm{A}$, e ancora di più nella zona $\mathrm{B}$, le tipologie di abitazione più diffuse sono quelle di dimensioni medie (tre e quattro locali). 
Figura 26. Distribuzione delle abitazioni disponibili per numero di vani - Zona B.

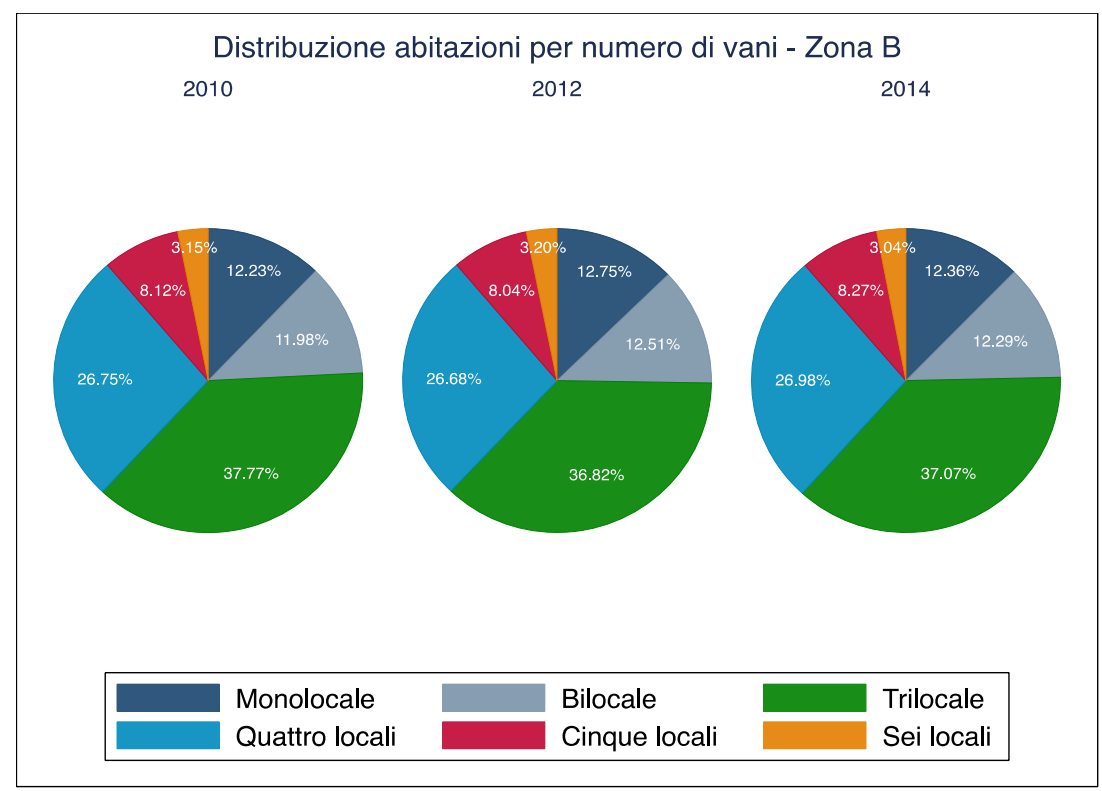

La Tabella 22 riporta il numero assoluto di abitazioni, suddivise per zone di applicazione del canone concordato e numero di vani, presenti nei diversi stock disponibili. Si noti come tendenzialmente nella Zona B le abitazioni di dimensione (e vani) maggiori risultino essere presenti in misura minore. Tale aspetto appare particolarmente di interesse per la nostra analisi, in quanto mira principale della ricerca è comprendere se vi siano spazi per le famiglie all'interno del mercato bolognese della locazione. Dal punto di vista delle dimensioni le famiglie richiedono spazi superiori, in particolare laddove vi siano bambini o nuclei estesi con anziani. Dati i relativamente bassi prezzi del canone concordato in Zona B non si esclude che, soprattutto per le metrature più ampie, escano dal canone concordato per essere trattate a mercato libero (come per altro lasciato intendere dagli incontri con gli esperti del settore).

Di tutte le abitazioni locate a canone concordato, indipendentemente dal numero di vani, nel 2014 il $14.82 \%$ si colloca nella zona di pregio, il 37.98\% nella A e il $47.20 \%$ nella B. Le percentuali sono pressoché analoghe anche per gli stock 2010 e 2012.

I valori suddivisi per vano fanno invece riferimento alla distribuzione all'interno della singola zona delle diverse tipologie abitative. Si tratta delle stesse percentuali riportate nelle figure precedenti, a cui qui vengono aggiunti i valori assoluti.

La Tabella 22 illustra efficacemente l'offerta di abitazioni, suddivise per tipologia, a canone concordato. Rispetto alla media cittadina, la zona B, per tutti gli stock, presenta una maggior concentrazione di abitazioni medie $(64.05 \%$ nel 2014 , valore simile alle percentuali degli altri stock) e un numero più basso di 5 e 6 locali (11.31\% in zona B contro il $18.62 \%$ del Comune di Bologna). Per la zona A i valori percentuali sono allineati con quelli cittadini; mentre per la zona di pregio si registra una 
percentuale inferiore di abitazioni a 3 o 4 vani (38.93\% contro il $56.90 \%$ per l'intero Comune) e una maggiore per le abitazioni di metratura maggiore ( $34.4 \%$ nella zona di pregio e $18.62 \%$ a livello cittadino).

Tabella 22. Numero di abitazioni locate a canone concordato, suddivise per zone di applicazione del canone concordato.

\begin{tabular}{|c|c|c|c|c|c|c|c|}
\hline \multicolumn{8}{|c|}{ Offerta di abitazioni a canone concordato } \\
\hline \multirow{2}{*}{$\begin{array}{l}\text { Zona } \\
\text { Canone }\end{array}$} & \multirow[t]{2}{*}{ Vani } & \multicolumn{2}{|c|}{2010} & \multicolumn{2}{|c|}{2012} & \multicolumn{2}{|c|}{2014} \\
\hline & & $\mathrm{N}$ & $\%$ & $\mathrm{~N}$ & $\%$ & $\mathrm{~N}$ & $\%$ \\
\hline \multirow{7}{*}{ 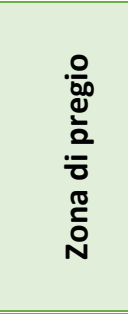 } & 1 & 468 & 15.54 & 536 & 17.18 & 628 & 16.82 \\
\hline & 2 & 287 & 9.53 & 318 & 10.20 & 368 & 9.86 \\
\hline & 3 & 624 & 20.72 & 640 & 20.52 & 767 & 20.55 \\
\hline & 4 & 560 & 18.60 & 576 & 18.47 & 686 & 18.38 \\
\hline & 5 & 437 & 14.51 & 423 & 13.56 & 517 & 13.85 \\
\hline & 6 & 635 & 21.09 & 626 & 20.07 & 767 & 20.55 \\
\hline & Tot & 3011 & 14.43 & 3119 & 15.25 & 3733 & 14.82 \\
\hline \multirow{7}{*}{ 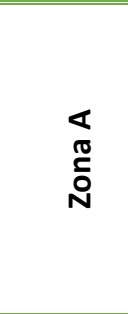 } & 1 & 966 & 12.04 & 989 & 12.62 & 1165 & 12.18 \\
\hline & 2 & 864 & 10.77 & 860 & 10.98 & 1074 & 11.23 \\
\hline & 3 & 2491 & 31.06 & 2452 & 31.30 & 2999 & 31.36 \\
\hline & 4 & 1916 & 23.89 & 1838 & 23.46 & 2265 & 23.68 \\
\hline & 5 & 963 & 12.01 & 929 & 11.86 & 1154 & 12.07 \\
\hline & 6 & 820 & 10.22 & 766 & 9.78 & 907 & 9.48 \\
\hline & Tot & 8020 & 38.43 & 7834 & 38.31 & 9564 & 37.98 \\
\hline \multirow{7}{*}{$\begin{array}{l}\infty \\
\mathbb{0} \\
\stackrel{\Sigma}{0}\end{array}$} & 1 & 1203 & 12.23 & 1211 & 12.75 & 1469 & 12.36 \\
\hline & 2 & 1179 & 11.98 & 1188 & 12.51 & 1461 & 12.29 \\
\hline & 3 & 3716 & 37.77 & 3497 & 36.82 & 4406 & 37.07 \\
\hline & 4 & 2632 & 26.75 & 2534 & 26.68 & 3207 & 26.98 \\
\hline & 5 & 799 & 8.12 & 764 & 8.04 & 983 & 8.27 \\
\hline & 6 & 310 & 3.15 & 304 & 3.20 & 361 & 3.04 \\
\hline & Tot & 9839 & 47.14 & 9498 & 46.44 & 11887 & 47.20 \\
\hline
\end{tabular}

Le abitazioni più grandi sono quelle di interesse per le famiglie numerose, generalmente più povere (in quanto le ristrettezze economiche portano le famiglie ad unire diversi nuclei - la cosiddetta famiglia estesa ampiamente studiata nella letteratura sul disagio socio economico $)^{32}$ rispetto ad altre, e la loro distribuzione sul territorio non è uniforme. La presenza di molte di queste abitazioni in Zona di Pregio le rende inadatte a queste famiglie, in quanto si tratta delle abitazioni con i canoni mensili più alti, difficilmente sostenibili dalle famiglie numerose meno abbienti. All'estremo opposto, la zona B, quella con i prezzi più accessibili per queste, registra le percentuali minori. È però importante sottolineare il peso relativo delle diverse zone: nel 2014 quella di pregio conta 3'733 abitazioni totali (1'284 alloggi a cinque o sei locali), mentre la zona B ne conta, sempre nel 2014, 11'887 (di cui 1'344 grandi).

${ }^{32}$ Si veda Gal (2010) 
Tabella 23. Numero di persone - studenti, coppie e famiglie - allocabili secondo l'offerta di abitazioni a canone concordato, suddivise per vani e zone di applicazione del canone concordato.

\begin{tabular}{|c|c|c|c|c|c|c|c|c|c|c|}
\hline \multirow[b]{2}{*}{$\stackrel{\text { ర }}{\stackrel{0}{0}}$} & \multirow[b]{2}{*}{ in } & \multicolumn{3}{|c|}{2010} & \multicolumn{3}{|c|}{2012} & \multicolumn{3}{|c|}{2014} \\
\hline & & 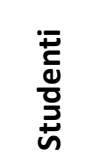 & $\frac{0}{\frac{0}{0}}$ & & 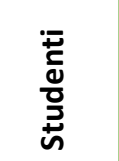 & $\frac{\frac{0}{0}}{\frac{0}{0}}$ & 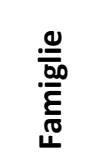 & 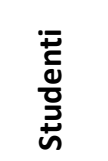 & $\frac{\frac{0}{0}}{\frac{0}{0}}$ & 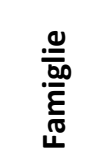 \\
\hline \multirow{7}{*}{ 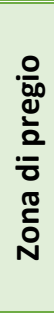 } & 1 & 468 & 936 & 936 & 536 & 1072 & 1072 & 628 & 1256 & 1256 \\
\hline & 2 & 574 & 574 & 574 & 636 & 636 & 636 & 736 & 736 & 736 \\
\hline & 3 & 1872 & 1248 & 861 & 1920 & 1280 & 1920 & 2301 & 1534 & 2301 \\
\hline & 4 & 2240 & 1120 & 1680 & 2304 & 1152 & 1728 & 2744 & 1372 & 2058 \\
\hline & 5 & 2185 & 874 & 1748 & 2115 & 846 & 1692 & 2585 & 1034 & 2068 \\
\hline & 6 & 3810 & 1270 & 2540 & 3756 & 1252 & 2504 & 4602 & 1534 & 3068 \\
\hline & Tot & 11149 & 6022 & 8339 & 11267 & 6238 & 9552 & 13596 & 7466 & 11487 \\
\hline \multirow{7}{*}{$\begin{array}{l}\varangle \\
\text { ¿ } \\
\stackrel{0}{0}\end{array}$} & 1 & 966 & 1932 & 1932 & 989 & 1978 & 1978 & 1165 & 2330 & 2330 \\
\hline & 2 & 1728 & 1728 & 1728 & 1720 & 1720 & 1720 & 2148 & 2148 & 2148 \\
\hline & 3 & 7473 & 4982 & 2592 & 7356 & 4904 & 7356 & 8997 & 5998 & 8997 \\
\hline & 4 & 7664 & 3832 & 5748 & 7352 & 3676 & 5514 & 9060 & 4530 & 6795 \\
\hline & 5 & 4815 & 1926 & 3852 & 4645 & 1858 & 3716 & 5770 & 2308 & 4616 \\
\hline & 6 & 4920 & 1640 & 3280 & 4596 & 1532 & 3064 & 5442 & 1814 & 3628 \\
\hline & Tot & 27566 & 16040 & 19132 & 26658 & 15668 & 23348 & 32582 & 19128 & 28514 \\
\hline \multirow{7}{*}{ 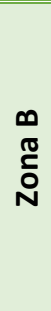 } & 1 & 1203 & 2406 & 2406 & 1211 & 2422 & 2422 & 1469 & 2938 & 2938 \\
\hline & 2 & 2358 & 2358 & 2358 & 2376 & 2376 & 2376 & 2922 & 2922 & 2922 \\
\hline & 3 & 11148 & 7432 & 3537 & 10491 & 6994 & 10491 & 13218 & 8812 & 13218 \\
\hline & 4 & 10528 & 5264 & 7896 & 10136 & 5068 & 7602 & 12828 & 6414 & 9621 \\
\hline & 5 & 3995 & 1598 & 3196 & 3820 & 1528 & 3056 & 4915 & 1966 & 3932 \\
\hline & 6 & 1860 & 620 & 1240 & 1824 & 608 & 1216 & 2166 & 722 & 1444 \\
\hline & Tot & 31092 & 19678 & 20633 & 29858 & 18996 & 18996 & 37518 & 23774 & 34075 \\
\hline
\end{tabular}

A livello complessivo, facendo riferimento ai dati 2014, la Zona B è quella che potenzialmente potrebbe allocare il maggior numero di persone, siano esse studenti, coppie o famiglie. Come per il Comune nel suo complesso, ancora una volta sono gli studenti coloro che registrano il numero più alto, anche se le famiglie registrano un valore solo leggermente inferiore. Le allocazioni disponibili nella Zona A sono inferiori di qualche migliaio, ma mai quanto quelle della Zona di Pregio, in cui il mercato a canone concordato può soddisfare la domanda di 13'596 studenti, 7'466 individui in coppia e 11'478 persone classificate come famiglia.

Dopo aver analizzato il territorio comunale nel suo complesso e aver sviluppato l'analisi per aree di applicazione del canone concordato, si conclude il capitolo presentando l'offerta di abitazioni a canone concordato per zone OMI.

La Figura 27 rappresenta la ripartizione delle abitazioni a canone concordato interamente considerato per zona $\mathrm{OMI}$, senza distinguere per dimensione. In appendice online sono invece riportate le mappe che rappresentano la stessa distribuzione tenendo conto della classificazione delle abitazioni per tipologia (monolocali, bilocali, trilocali, quadrilocali, cinque locali, sei locali). 
Figura 27. Numero abitazioni a canone concordato suddiviso per zone OMI.

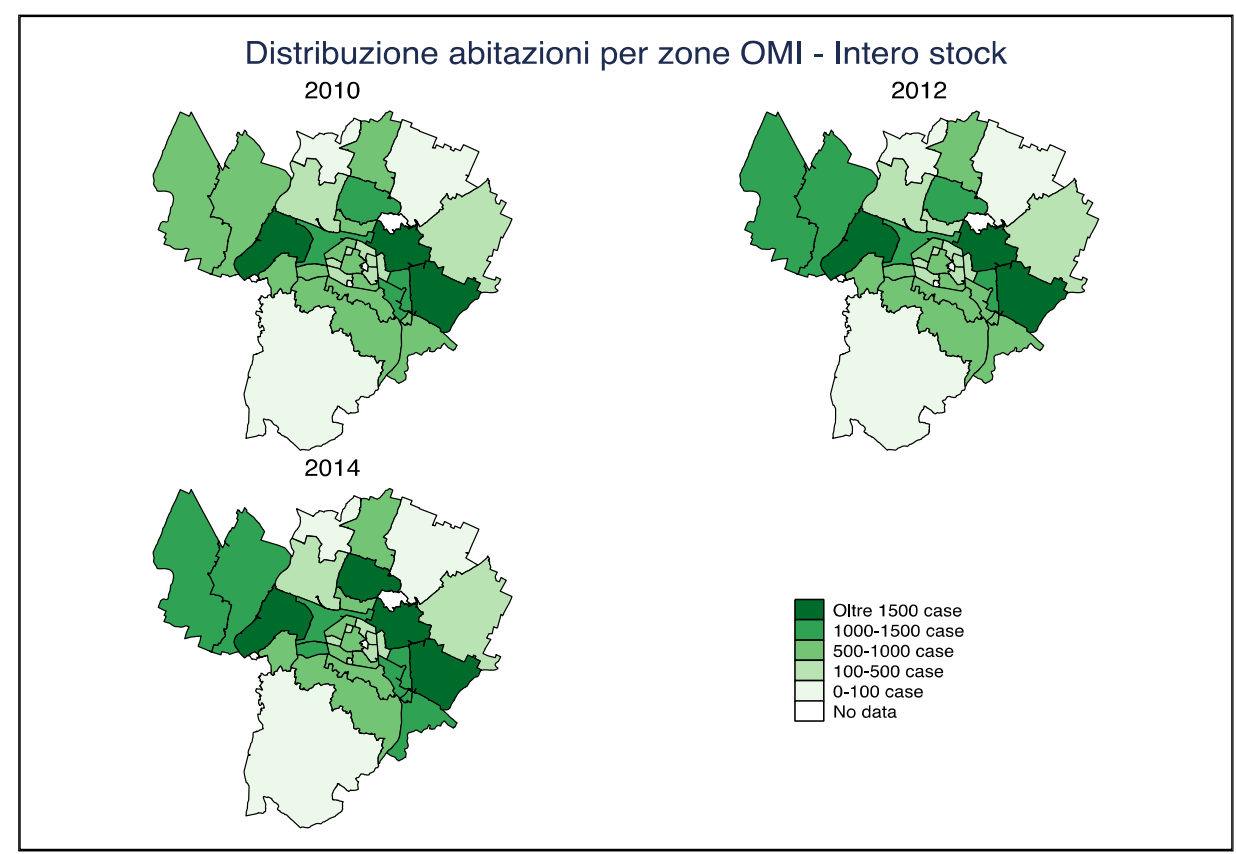

L'offerta di affitti a canone concordato non si distribuisce uniformemente su tutto il territorio cittadino, come mostrano le diverse gradazioni delle zone OMI.

Escludendo la zona D6 - Fiera, per cui non disponiamo di osservazioni, la zona collinare meridionale e alcune delle zone settentrionali centro - orientali risultano povere di offerta (tra 0 e 100 alloggi). È interessante notare come nessuna delle zone con il maggior numero di abitazioni (tra 1000 e 1500 e oltre le 1500) appartenga al centro storico. Ciò è probabilmente dovuto alla bassa superficie delle zone centrali. Le zone che presentano il maggior numero assoluto di alloggi presenti sul mercato a canone concordato (oltre le 1500 abitazioni per zona) sono: D20 - "Saffi - Santa Viola - Barca", D4 - "Mazzini - Fossolo", D5 - "San Donato", per tutti gli anni considerati, e anche la zona D8 - "Bolognina - Arcoveggio" nel 2014.

Le mappe più dettagliate, relative alla quantità di abitazioni disponibili nelle differenti Zone OMI, suddivise per numero di vani, sono fornite nell'appendice online. A questo proposito si può comunque sinteticamente affermare che le diverse tipologie di abitazioni non sono uniformemente distribuite sul territorio. Le zone del centro storico, ad esempio, presentano, rispetto al resto della città, una discreta offerta di abitazioni piccole e grandi, mentre quelle medie non registrano valori maggiori rispetto al resto della città. La maggior concentrazione di monolocali si registra, per tutti gli anni, nelle zone D20 - "Saffi - Santa Viola - Barca" e D4 - "Mazzini - Fossolo", oltre che nella zona centrale B1 per il 2014. Le stesse zone, oltre alla D5 - "San Donato" e alla D8 - "Bolognina - Arcoveggio" nel 2014 sono quelle a maggior intensità d'offerta anche per i bilocali. 
Le abitazioni di medie dimensioni (trilocali) registrano valori assoluti di offerta elevati lungo entrambe le direzioni della via Emilia, come è ben visibile dalla mappa del 2014. In questo caso anche alcune zone periferiche appartenenti all' area B di applicazione del canone concordato presentano le gradazioni più scure. Va precisato che per i tri e quadrilocali, la gradazione più scura rappresenta un numero di alloggi offerti a canone concordato nella zona maggiore a 400, diversamente dalle mappe relative alle abitazioni più piccole in cui la fascia maggiore ha come limite inferiore 200. Infine, l'offerta di abitazioni di dimensioni maggiori (5 vani), è più bassa negli stock in esame, come evidenzia il valore minimo della fascia maggiore fissato a 150 . In questo caso le zone con la presenza maggiore di alloggi a canone concordato sono quelle SudOrientali rispetto alla cinta muraria della città. 


\section{I prezzi degli affitti al metro quadro}

\section{I valori mensili a metro quadro a canone concordato}

Oggetto di questa sezione dell'analisi è l'andamento dei valori al metro quadro per le locazioni a canone concordato e a canone di mercato.

La Tabella 24 riporta i valori mensili al metro quadro per i contratti a canone concordato, in base agli accordi tra rappresentanze dei proprietari immobiliari e dei conduttori siglati nel 2008 e nel 2013, ed entrati in vigore nell'anno successivo. Importante sottolineare che i valori indicati nel 2013 rimangono identici a quattro anni di distanza: il nuovo accordo dell'autunno 2017, infatti, congela i prezzi stabiliti in precedenza. Nel dettaglio, i prezzi stessi risentono di tre fattori:

- la collocazione dell'immobile in una delle tre zone previste;

- il numero di vani dell'abitazione;

- la presenza di elementi di pregio.

Tabella 24 - Valori massimi e minimi al MQ per gli accordi a canone concordato 2008 e 2013, per zona e numero di vani; scarto percentuale 2008-2013. Si ricorda che i prezzi 2013 permangono identici nel successivo accordo 2017.

\begin{tabular}{|c|c|c|c|c|c|c|c|}
\hline \multirow[t]{2}{*}{ Zone } & \multirow[t]{2}{*}{ Vani } & \multicolumn{2}{|l|}{2008} & \multicolumn{2}{|c|}{2013 (2017) } & \multirow{2}{*}{$\begin{array}{l}\Delta \text { Massimi } \\
2008-2013(\%)\end{array}$} & \multirow{2}{*}{$\begin{array}{l}\Delta \text { Minimi } \\
\text { 2008-13 (\%) }\end{array}$} \\
\hline & & $\operatorname{Max}(€)$ & $\operatorname{Min}(€)$ & $\operatorname{Max}(€)$ & $\operatorname{Min}(€)$ & & \\
\hline \multirow{6}{*}{ 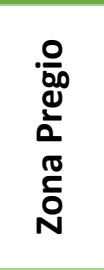 } & 1 & 14.25 & 7.75 & 13.17 & 7.17 & -7.6 & -7.5 \\
\hline & 2 & 12.42 & 6.75 & 11.50 & 6.25 & -7.4 & -7.4 \\
\hline & 3 & 10.83 & 5.83 & 10.00 & 5.42 & -7.7 & -7.1 \\
\hline & 4 & 9.83 & 5.58 & 9.08 & 5.17 & -7.6 & -7.5 \\
\hline & 5 & 8.83 & 5.00 & 8.17 & 4.67 & -7.5 & -6.7 \\
\hline & 6 & 7.92 & 4.50 & 7.33 & 4.17 & -7.4 & -7.4 \\
\hline \multirow{6}{*}{$\begin{array}{l}\varangle \\
\mathbb{\sigma} \\
\text { ¿ } \\
\text { N }\end{array}$} & 1 & 11.92 & 6.42 & 11.00 & 5.92 & -7.7 & -7.8 \\
\hline & 2 & 10.25 & 5.50 & 9.50 & 5.08 & -7.3 & -7.6 \\
\hline & 3 & 9.00 & 4.75 & 8.33 & 4.42 & -7.4 & -7.0 \\
\hline & 4 & 8.17 & 4.58 & 7.58 & 4.25 & -7.1 & -7.3 \\
\hline & 5 & 7.42 & 4.17 & 6.83 & 3.83 & -7.9 & -8.0 \\
\hline & 6 & 6.58 & 3.67 & 6.08 & 3.42 & -7.6 & -6.8 \\
\hline \multirow{6}{*}{ 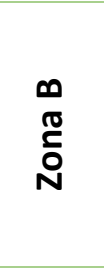 } & 1 & 10.17 & 5.50 & 9.17 & 5.00 & -9.8 & -9.1 \\
\hline & 2 & 8.83 & 4.75 & 8.00 & 4.33 & -9.4 & -8.8 \\
\hline & 3 & 7.75 & 4.17 & 7.00 & 3.75 & -9.7 & -10.0 \\
\hline & 4 & 7.00 & 4.00 & 6.33 & 3.67 & -9.5 & -8.3 \\
\hline & 5 & 6.33 & 3.58 & 5.75 & 3.25 & -9.2 & -9.3 \\
\hline & 6 & 5.67 & 3.25 & 5.17 & 3.00 & -8.8 & -7.7 \\
\hline \multicolumn{6}{|l|}{ media } & -8.1 & -7.8 \\
\hline
\end{tabular}

I valori riportati nella tabella riflettono efficacemente i primi due fattori. L'assenza di informazioni sulla distribuzione dei suddetti elementi di pregio, invece, ha comportato 
la scelta dei soli parametri estremi (divisi su dodici mensilità) - dove il massimo esprime la più alta dotazione degli stessi, e il minimo la più bassa ${ }^{33}$.

Si noti in primis il calo dei prezzi impresso dall'accordo 2013, leggermente più rilevante sui valori massimi $(-8.1 \%)$ che sui minimi (-7.8\%). In particolare, si sottolinea che il decremento è avvenuto in misura maggiore per la "Zona B" (-9.4\% i massimi e $8.9 \%$ i minimi). Una zona, questa, ampia e variegata che include i quartieri più popolari e densamente abitati (nonché più poveri - Bolognina, Pilastro, Barca, Borgo Panigale), e la fascia rurale di pianura (mediamente più ricca e a minore intensità residenziale fatta esclusione per l'area statistica di San Savino) ${ }^{34}$. In seconda battuta, il calo dei parametri all'aumentare del numero di vani, finalizzato a replicare la dinamica del mercato (per il quale i prezzi al metro quadro tendono a decrescere all'aumentare delle metrature e delle stanze riflettendo una minore domanda di mercato) può, in linea di principio, generare dinamiche di prezzo irrealistiche in casi di estrema discordanza tra ampiezza e numero di stanze per particolari tipi di alloggio quali, ad esempio, i cosiddetti Loft.

\section{I valori mensili a metro quadro a canone di mercato}

Prima di analizzare i valori mensili a metro quadro a canone di mercato, occorre premettere che le rilevazioni dell'OMI, ossia le uniche informazioni disponibili a questo livello di dettaglio e costanza nel tempo, si basano sul complesso dei contratti sottoscritti in una determinata zona. In una città quale Bologna, dove circa metà delle locazioni avvengano a canone concordato, è inevitabile che queste influiscano sui valori complessivi che noi definiamo "di mercato". È lecito attendersi dunque che $i$ prezzi al metro quadro a canone libero siano tendenzialmente superiori a quelli di cui disponiamo. Questo fattore è sicuramente rilevante nell'analisi, tuttavia, in assenza di informazione disaggregata sulle tipologie contrattuali sottoscritte nelle singole zone, non si può far altro che utilizzare i valori OMI. Sintetizzando, i valori OMI sottostimano i reali prezzi di libero mercato.

Le Tabella 25 e Tabella 26 riportano i valori mensili al metro quadro - rispettivamente minimi e massimi - a canone di mercato, come da rilevazioni OMI. Ricordiamo che: (a) il riferimento geografico sono le trentadue aree omogenee in cui l'osservatorio divide Bologna; (b) i valori sono relativi al secondo semestre di ogni anno (escluso il 2017); (c) in tabella sono raffigurate medie tra i prezzi di locazione delle varie tipologie di edificio ad uso abitativo registrate ${ }^{35}$. Entrambe le tabelle presentano agli estremi dell'arco temporale di nostro interesse i valori espressi in euro, mentre per ogni anno è indicata la variazione percentuale sul precedente. Si consideri che la mancanza di dati (da imputare all'assenza di rilevazioni OMI per l'area omogenea considerata) è

\footnotetext{
${ }^{33}$ Si veda la sezione "Il calcolo dei prezzi a canone concordato" della Nota Metodologica.

${ }^{34}$ Si veda il capitolo 1, nella sezione "Una descrizione di Bologna nelle sue 90 aree statistiche".

${ }^{35}$ Si veda la sezione "Il calcolo dei prezzi a canone di mercato" della Nota Metodologica.
} 
indicata dalla sigla "nd"; invece le celle che non riportano valori ma un semplice trattino segnalano che non si registrano variazioni percentuali sull'anno precedente.

Osservando le tabelle, si nota un calo sensibile dei valori di locazione al metro quadro, più significativo nei massimi $(-22 \%)$ che nei minimi $(-16 \%)$. Senza addentrarsi ora nelle specificità di ogni singola zona, dove particolari interventi edilizi o investimenti in riqualificazione possono alterare il quadro rispetto alla tendenza generale, possiamo comunque segnalare una sostanziale stabilità dei prezzi fino al 2012. È invece dal 2013 , e soprattutto dal 2014 , che si avverte un consistente calo, in parte dovuto al recepimento nel monte complessivo degli accordi di locazione dell'abbassamento dei valori dei canoni concordati.

La tendenza del mercato ad essere trascinata dal canone concordato conferma da un lato la validità dell'utilizzo dello stesso al fine di calmierare gli affitti, dall'altro richiede una riflessione sullo stesso. La bassa differenza tra canone concordato e canone di mercato su varie tipologie abitative richiede un approfondimento. Se i prezzi sono pressoché uguali tra canone concordato e canone di mercato a meno della tassazione, infatti, semplicemente possiamo essere di fronte a due possibilità: o non vi è co partecipazione e i locatori stanno semplicemente ottenendo lo stesso profitto o il canone concordato è così concorrenziale dall'aver assorbito tutto il mercato della locazione a canone libero. Sebbene sulla carta questa seconda ipotesi possa apparire positiva, essa potrebbe essere un campanello di allarme che indica un livello di canoni concordati troppo elevati. Va infine notato un ulteriore punto: la molto minore incidenza nelle aree popolari di canoni concordati lascia presupporre che in suddette aree il canone concordato non sia sufficientemente concorrenziale; l'identificazione del prezzo di equilibrio ottimale è d'altronde particolarmente complessa.

L'influenza dei prezzi a canone concordato sui valori complessivi OMI è testimoniata anche dalla Tabella 27, che rivela una correlazione complessiva piuttosto stretta ( $\rho>0.4$ ) sia nei minimi che nei massimi, la quale si attesta su valori simili anche se verificata anno per anno (pur essendo il dato per 2009, 2011 e 2014 leggermente inferiore).

Per una più precisa valutazione, sarebbe opportuno poter stimare il peso del mercato a canone concordato sul numero totale dei contratti d'affitto sottoscritti. Al momento della pubblicazione dell'accordo 2017 tra sindacati di inquilini e proprietari, stando ad un comunicato diffuso dal Comune di Bologna, gli affitti a canone concordato erano "oltre la metà dei contratti di locazione attivi complessivamente in città"36. Non è possibile, tuttavia, usufruire di informazioni più precise.

\footnotetext{
${ }^{36}$ Fonte: “Rinnovato l'Accordo per l'applicazione dei contratti d'affitto a canone concordato: sarà valido in tutti i comuni della Città metropolitana", Comune di Bologna, 26 Settembre 2017, consultabile al link: http://www.comune.bologna.it/news/rinnovato-l-accordo-l-applicazione-deicontratti-d-affitto-canone-concordato-sar-valido-tutti-i
} 
Tabella 25 - Valori minimi al MQ a canone di mercato nelle trentadue aree omogenee OMI per gli anni 2009-2017; variazioni \% sull'anno precedente (dal 2010 al 2017); scarto sul periodo. ${ }^{37}$

\begin{tabular}{|c|c|c|c|c|c|c|c|c|c|c|c|}
\hline & MIN & Varia & oni $(\%$ & sull'a & nno pr & eceder & & & & Mas & \\
\hline & $\begin{array}{l}(€) \\
2009\end{array}$ & 옥 & 겅 & $\stackrel{\sim}{\sim}$ & $\stackrel{m}{\stackrel{m}{\circ}}$ & 공 & 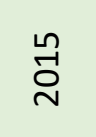 & 붕 & 공 & $\begin{array}{l}(€) \\
2017\end{array}$ & $\begin{array}{l}2017- \\
2009 \\
(\%)\end{array}$ \\
\hline B1 & 8.92 & - & -0.4 & -0.2 & -7.9 & -15.5 & - & - & - & 6.90 & -23 \\
\hline B7 & 8.50 & - & - & - & -5.9 & -6.3 & - & - & -10.0 & 6.75 & -21 \\
\hline C10 & 8.50 & -11.8 & - & - & -6.7 & -10.0 & 19.1 & - & -6.7 & 7.00 & -18 \\
\hline C11 & 9.50 & -5.3 & - & -3.9 & -10.4 & -5.2 & -15.0 & - & -4.0 & 6.00 & -37 \\
\hline C3 & 9.50 & - & - & - & -3.7 & -12.6 & -6.3 & - & -6.7 & 7.00 & -26 \\
\hline C4 & 8.75 & - & - & -5.7 & -6.1 & -9.7 & - & - & - & 7.00 & -20 \\
\hline C5 & 8.75 & - & - & -5.7 & -4.9 & -5.7 & - & - & -3.4 & 7.15 & -18 \\
\hline C6 & 8.50 & - & - & - & -2.4 & -9.6 & - & - & - & 7.50 & -12 \\
\hline C7 & 8.00 & - & - & - & -3.1 & -4.5 & - & - & - & 7.40 & -8 \\
\hline C8 & 8.25 & - & - & -3.0 & -8.1 & -6.8 & -5.1 & -1.5 & - & 6.40 & -22 \\
\hline C9 & 8.50 & - & - & - & -4.1 & -8.0 & - & - & - & 7.50 & -12 \\
\hline D16 & 8.50 & - & - & - & - & -11.8 & -6.7 & - & - & 7.00 & -18 \\
\hline D17 & 7.75 & - & - & 1.3 & -3.8 & -2.7 & -4.8 & - & - & 7.00 & -10 \\
\hline D19 & 7.00 & - & - & 0.7 & -2.8 & -5.1 & - & - & - & 6.50 & -7 \\
\hline D2 & 6.75 & - & - & -3.7 & -2.3 & -3.9 & - & - & - & 6.10 & -10 \\
\hline D20 & 6.00 & - & - & - & -1.7 & -8.5 & - & 13.0 & -15.6 & 5.15 & -14 \\
\hline D21 & 8.13 & - & - & -0.6 & -10.2 & -13.8 & - & - & - & 6.25 & -23 \\
\hline D22 & 6.00 & - & - & -1.7 & - & - & -19.5 & - & - & 4.75 & -21 \\
\hline D23 & 7.25 & - & - & - & -3.5 & -4.3 & -21.6 & - & - & 5.25 & -28 \\
\hline D24 & 7.35 & - & - & -1.5 & -5.4 & 13.1 & -6.5 & - & - & 7.25 & -1 \\
\hline D25 & 6.50 & - & - & - & -4.6 & -9.7 & - & -4.5 & - & 5.35 & -18 \\
\hline D3 & 6.75 & - & - & -1.5 & -6.0 & -5.6 & -1.7 & - & - & 5.80 & -14 \\
\hline D4 & 6.75 & - & - & -2.9 & -2.3 & -7.8 & - & - & - & 5.90 & -13 \\
\hline D5 & 6.50 & - & - & -1.5 & -4.7 & - & - & - & - & 6.10 & -6 \\
\hline D7 & 6.25 & -3.2 & - & -2.5 & - & -2.5 & -8.7 & - & - & 5.25 & -16 \\
\hline D8 & 6.00 & -4.2 & - & -4.3 & -3.6 & -1.9 & - & 3.9 & 1.9 & 5.50 & -8 \\
\hline D9 & 5.75 & -2.6 & - & -1.8 & -13.6 & -2.1 & 12.9 & -1.9 & - & 5.15 & -10 \\
\hline E1 & 6.25 & -1.6 & - & -0.4 & -1.63 & -0.41 & nd & nd & nd & nd & \\
\hline R1 & 4.63 & - & - & -1.1 & -1.09 & 8.29 & nd & nd & nd & nd & \\
\hline R2 & 4.00 & - & - & - & - & -7.50 & nd & nd & nd & nd & \\
\hline R3 & nd & nd & nd & nd & nd & nd & nd & nd & nd & nd & \\
\hline R4 & 7.50 & - & - & - & -1.3 & -3.4 & nd & nd & nd & nd & \\
\hline
\end{tabular}

37 È riportata la sigla "nd" in caso di mancanza di dati relativi ai prezzi di locazione di immobili ad uso abitativo all'interno delle rilevazioni dell'Osservatorio del Mercato Immobiliare. L'assenza di variazioni sull'anno precedente è segnalata da un trattino. 
Tabella 26 - Valori massimi al MQ a canone di mercato nelle trentadue aree omogenee OMI per gli anni 2009-2017; variazioni \% sull'anno precedente (dal 2010 al 2017); scarto sul periodo. ${ }^{38}$

\begin{tabular}{|c|c|c|c|c|c|c|c|c|c|c|c|}
\hline & MA & Varia & ni (c & sull': & inno p & recede & te & & & MAY & \\
\hline & $\begin{array}{l}(€) \\
2009\end{array}$ & 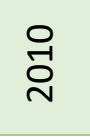 & $\underset{\sim}{\stackrel{ }{\circ}}$ & $\underset{\sim}{\stackrel{\sim}{\circ}}$ & 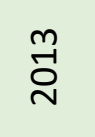 & $\underset{\sim}{\stackrel{\leftrightarrow}{\circ}}$ & 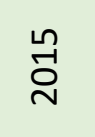 & $\begin{array}{l}\bullet \\
\stackrel{-}{O} \\
\stackrel{2}{ }\end{array}$ & 공 & $\begin{array}{l}\text { (€) } \\
2017\end{array}$ & $\begin{array}{l}2017- \\
2009 \\
(\%)\end{array}$ \\
\hline B1 & 13.83 & - & - & - & -2.4 & -9.3 & - & - & -12.2 & 10.75 & -22 \\
\hline B7 & 12 & - & - & -1.3 & - & 1.3 & - & - & -20.8 & 9.5 & -21 \\
\hline C10 & 12 & -4.2 & - & - & - & -15.2 & 2.6 & 10.0 & -18.2 & 9 & -25 \\
\hline C11 & 12.75 & -5.9 & - & -2.1 & - & -14.9 & -12.5 & - & -8.6 & 8 & -37 \\
\hline C3 & 13 & - & - & - & - & -17.3 & -14.0 & - & -8.1 & 8.5 & -35 \\
\hline C4 & 12 & - & - & - & - & -16.7 & -2.5 & - & - & 9.75 & -19 \\
\hline C5 & 12 & - & - & - & - & -10.4 & - & - & 2.3 & 11 & -8 \\
\hline C6 & 14.5 & - & - & - & - & -8.6 & -6.8 & - & - & 12.35 & -15 \\
\hline C7 & 12 & - & - & - & - & -14.6 & - & - & - & 10.25 & -15 \\
\hline C8 & 12 & - & - & - & -4.2 & -8.7 & -9.5 & - & - & 9.5 & -21 \\
\hline C9 & 13 & - & - & - & - & -11.5 & -8.7 & - & - & 10.5 & -19 \\
\hline D16 & 13.5 & - & - & -5.6 & - & -21.6 & -12.5 & - & - & 8.75 & -35 \\
\hline D17 & 13.75 & - & - & -5.5 & -3.9 & -4.0 & -2.1 & - & - & 11.75 & -15 \\
\hline D19 & 11.25 & - & - & -3.3 & -2.3 & -5.9 & -17.5 & -1.2 & - & 8.15 & -28 \\
\hline D2 & 10.5 & - & - & - & -4.8 & -9.0 & -1.7 & - & - & 8.95 & -15 \\
\hline D20 & 8.67 & - & -0.2 & -0.2 & - & -17.8 & - & 1.4 & -13.2 & 6.25 & -28 \\
\hline D21 & 12.25 & - & - & - & -6.1 & -13.0 & -10.0 & - & -6.7 & 8.4 & -31 \\
\hline D22 & 9.25 & - & - & - & -5.4 & -9.7 & -17.7 & - & - & 6.5 & -30 \\
\hline D23 & 12 & - & - & - & - & -4.2 & -28.3 & -6.1 & - & 7.75 & -35 \\
\hline D24 & 12.3 & - & -0.3 & - & -2.9 & 13.5 & -5.6 & - & - & 12.75 & 4 \\
\hline D25 & 10 & - & -0.5 & - & -5.8 & -12.0 & - & -10.3 & - & 7.4 & -26 \\
\hline D3 & 9.75 & - & - & - & -5.1 & -10.8 & -3.0 & - & - & 8 & -18 \\
\hline D4 & 9 & - & - & - & -2.8 & -2.9 & -5.9 & - & - & 8 & -11 \\
\hline D5 & 9.75 & - & - & - & -3.6 & -9.6 & -1.8 & -2.4 & - & 8.15 & -16 \\
\hline D7 & 10.75 & - & - & - & -2.3 & -14.3 & -19.4 & - & - & 7.25 & -33 \\
\hline D8 & 8.5 & - & - & - & -5.9 & -8.1 & - & 2.0 & - & 7.5 & -12 \\
\hline D9 & 9 & -5.6 & - & - & -11.8 & -9.3 & 10.3 & -3.3 & - & 7.25 & -19 \\
\hline E1 & 8.75 & -2.3 & -0.3 & - & -1.5 & -5.9 & nd & nd & nd & nd & -22 \\
\hline R1 & 7.63 & - & -0.7 & - & -1.3 & -1.7 & nd & nd & nd & nd & \\
\hline R2 & 7.00 & - & - & - & - & -11.4 & nd & nd & nd & nd & \\
\hline R3 & nd & nd & nd & nd & nd & nd & nd & nd & nd & nd & \\
\hline R4 & 12.00 & - & - & - & - & -18.7 & nd & nd & nd & nd & \\
\hline
\end{tabular}

38 È riportata la sigla "nd" in caso di mancanza di dati relativi ai prezzi di locazione di immobili ad uso abitativo all'interno delle rilevazioni dell'Osservatorio del Mercato Immobiliare. L'assenza di variazioni sull'anno precedente è segnalata da un trattino. 
Tabella 27 - Correlazione tra valori al MQ a canone concordato e a canone di mercato ( $\rho)$.

\begin{tabular}{|l|c|c|c|c|c|c|c|c|c|c|}
\hline \multicolumn{1}{c|}{ Correlazione prezzi al MQ a canone concordato e a mercato } \\
\hline & 2009 & 2010 & 2011 & 2012 & 2013 & 2014 & 2015 & 2016 & 2017 & Totale \\
\hline Valori minimi & 0.29 & 0.39 & 0.30 & 0.41 & 0.38 & 0.34 & 0.38 & 0.36 & 0.36 & 0.41 \\
\hline Valori massimi & 0.30 & 0.38 & 0.34 & 0.38 & 0.37 & 0.32 & 0.40 & 0.36 & 0.37 & 0.42 \\
\hline
\end{tabular}

Se il 2013 prima, e il 2014 poi sono stati gli anni di un calo dei valori diffuso in tutte le aree omogenee $\mathrm{OMI}$, benché in misura più o meno intensa, meno uniforme è la tendenza per gli anni successivi.

Come esplicitato dalla Tabella 28, il 2015 vede i valori massimi ancora in discesa (seppur in maniera diversificata da zona a zona), mentre i minimi si avviano ad una stabilizzazione in circa due terzi delle aree considerate (con la media degli scarti fortemente influenzata dai drastici abbassamenti dei valori di tre zone, di fatto posticipati rispetto al 2014). II 2016 e il 2017, infine, sono anni di progressiva stabilizzazione: una tendenza, questa, violata pressoché esclusivamente da un nuovo calo registrato in gran parte del centro storico (più precisamente le aree centrali B1 e $\mathrm{B} 7$, e quelle più a nord, interne al perimetro delle mura).

Sono, d'altronde, proprio le aree del centro storico ad aver subito l'abbassamento più drastico dei valori al metro quadro per le locazioni, tanto rispetto ai minimi che ai massimi (in particolare per la zona C11 "Quartiere Marconi: S. Felice - Grada - Vicini Pietramellara - Indipendenza - Falegnami - Galliera - Mille - Marconi" e C3 "Irnerio Centotrecento - Moline - Righi - P.zza VIII Agosto - Indipendenza - V.li Masini e Pichat"). Meno omogeneo l'andamento fuori le mura, dove i cali più sensibili si registrano sui valori massimi; in questo contesto, comunque, meritano una menzione le zone D16 ("Pedecollinare"), D21 ("Andrea Costa - Saragozza"), D22 ("Corticella"), D23 ("V.le Filopanti - Massarenti - Zanolini - Stazione S. Vitale"), dove lo scarto da inizio a fine periodo è significativamente maggiore della media, sia nei minimi che nei massimi.

Tabella 28 - Media e deviazione standard degli scarti percentuali sull'anno precedente, dei valori di locazione (al MQ) a canone di mercato.

\begin{tabular}{|c|c|c|c|c|c|c|c|c|c|}
\hline & & 2010 & 2011 & 2012 & 2013 & 2014 & 2015 & 2016 & 2017 \\
\hline \multirow[t]{2}{*}{$\begin{array}{l}\text { Valori } \\
\text { minimi }\end{array}$} & $\begin{array}{l}\text { Media scarti su } \\
\text { anno } \\
\text { precedente (\%) }\end{array}$ & -0.92 & -0.01 & -1.29 & -4.25 & -5.63 & -2.36 & 0.33 & -1.65 \\
\hline & St. dev. & 2.39 & 0.07 & 1.81 & 3.25 & 5.79 & 7.94 & 2.76 & 3.80 \\
\hline \multirow[t]{2}{*}{$\begin{array}{l}\text { Valori } \\
\text { massimi }\end{array}$} & $\begin{array}{l}\text { Media scarti su } \\
\text { anno } \\
\text { precedente (\%) }\end{array}$ & -0.58 & -0.06 & -0.58 & -2.32 & -9.76 & -6.17 & -0.36 & -3.17 \\
\hline & St. dev. & 1.58 & 0.16 & 1.47 & 2.76 & 6.69 & 8.15 & 3.13 & 6.11 \\
\hline
\end{tabular}


Nelle pagine successive, la Figura 28 e la Figura 29 trasferiscono graficamente su una mappa i valori di affitto al metro quadro in tre anni chiave. ${ }^{39}$

Figura 28. Valori minimi al MQ a canone di mercato nelle aree omogenee OMI per gli anni 2009, 2014 e 2017.

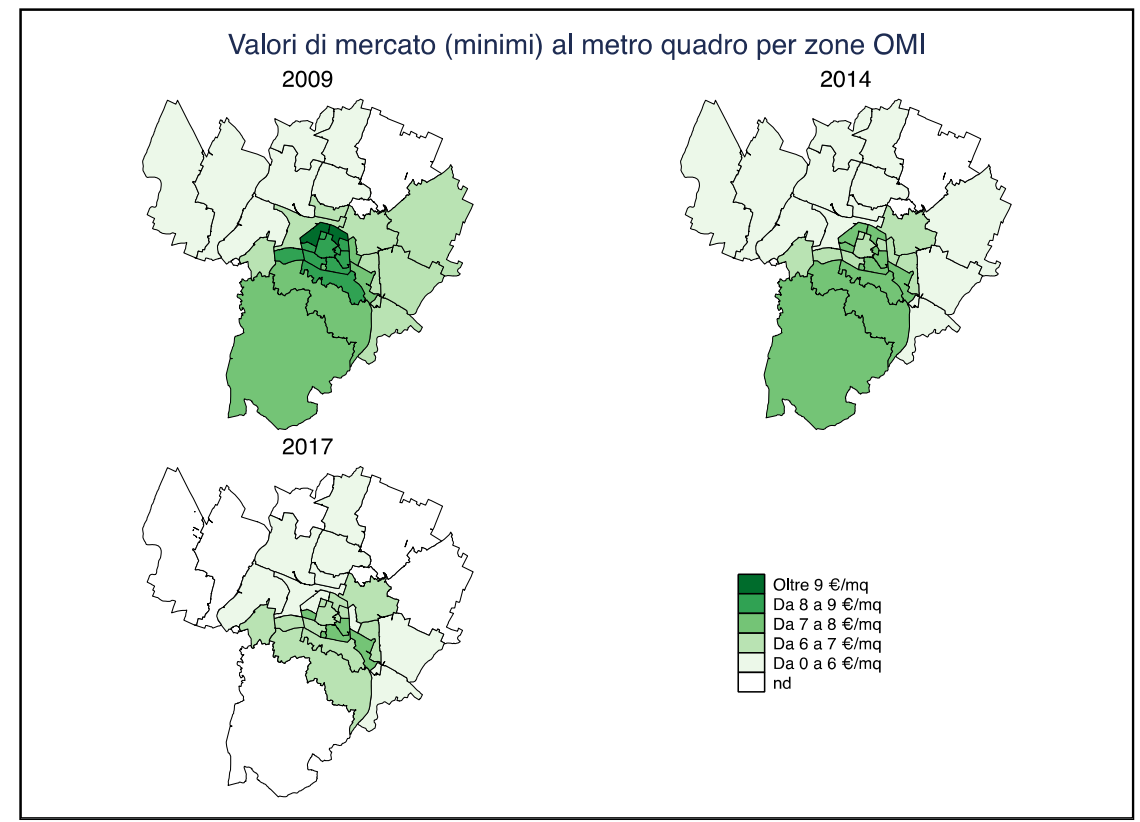

Figura 29. Valori massimi al MQ a canone di mercato nelle aree omogenee OMI per gli anni 2009, 2014 e 2017.

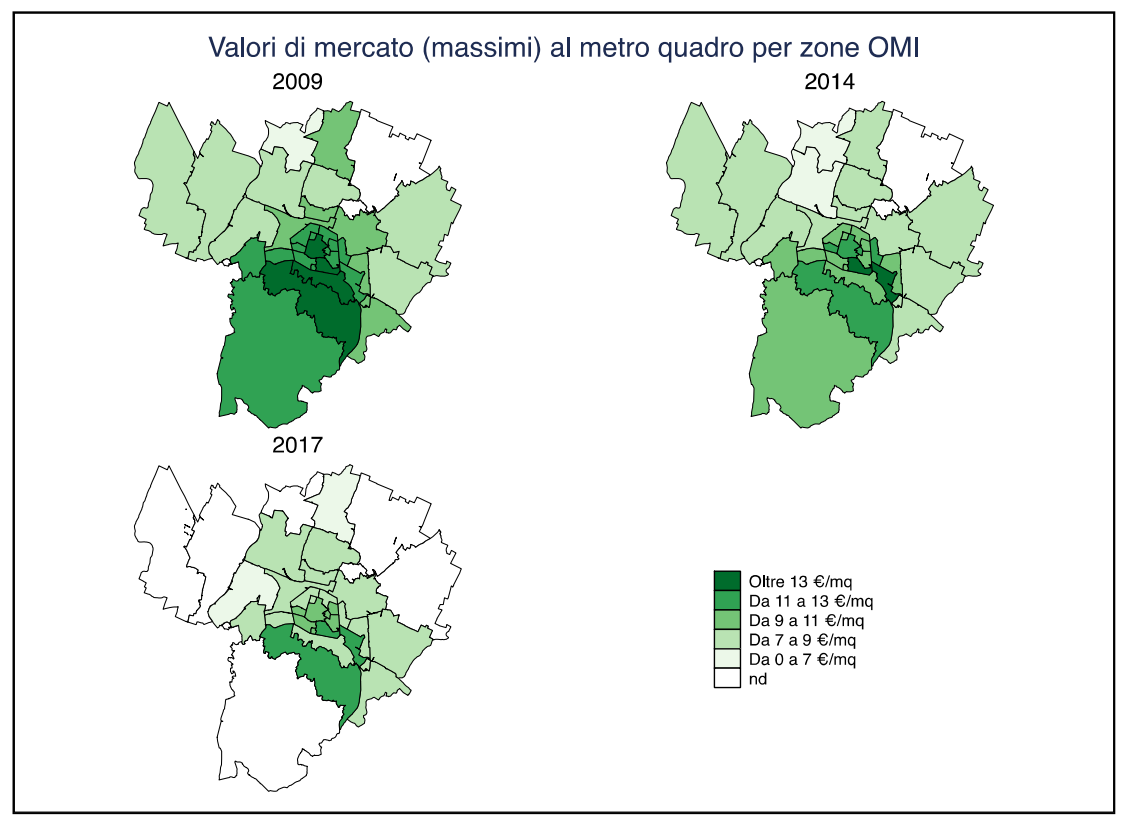

${ }^{39}$ Si segnala inoltre che per il 2017 OMI non rileva i valori al MQ nelle zone rurali: a questo si deve la presenza, ai margini della città, di numerose aree bianche. 

Bologna.

In questo paragrafo si presenta l'evoluzione temporale, lungo il periodo 2009-2017, dei prezzi al metro quadro a canone di mercato e a canone concordato. Per ogni tipologia contrattuale sono rappresentati il prezzo massimo e minimo, in modo da poter offrire un'informazione più ricca rispetto ad un unico indice sintetico. L'analisi è suddivisa per le tre aree geografiche di canone concordato: Zona di Pregio, Zona A e Zona B. All'interno di ogni zona si scompone ulteriormente per tipologia di abitazione, adottando una definizione estensiva e non mutuamente esclusiva del numero di vani. ${ }^{40}$ All'interno di ogni zona, il prezzo locativo di mercato al metro quadro è lo stesso per tutte le tipologie di abitazione considerate, in quanto l'OMI non fornisce quotazioni distinte in questo senso. I valori massimo e minimo di mercato sono calcolati come media dei prezzi tra tutte le abitazioni presenti nel dataset, collocate nella zona di applicazione del canone concordato di riferimento.

Diversamente, il valore di locazione a canone concordato si differenzia per definizione non solo a seconda della collocazione dell'immobile, ma anche del numero di vani. Conseguentemente, nei grafici seguenti, soltanto le serie storiche relative a quest'ultima forma contrattuale varieranno in funzione del numero di stanze. Si riporta inoltre il totale delle abitazioni per numero di vani, per meglio definire lo stock di abitazioni considerato, rispetto al quale vengono calcolati il valore medio, massimo e minimo dei prezzi di mercato. È possibile infatti che una variazione dei prezzi possa essere in parte dovuta ad una diversa composizione della popolazione di riferimento che, ricordiamo, è costituita dagli immobili in regime di canone concordato negli anni 2010, 2012 e $2014 .{ }^{41}$

Uno degli aspetti su cui questa sezione si sofferma è il confronto tra i livelli dei prezzi per le due forme di contratti di locazione. Infatti, poiché l'introduzione del canone concordato aveva l'obiettivo di rendere più semplice per le famiglie l'accesso alla casa, è fondamentale capire se i prezzi a canone concordato siano effettivamente inferiori rispetto a quelli di mercato.

I grafici riportati in Figura 31, Figura 33 e Figura 35 si riferiscono a Bologna, nelle sue tre fasce di applicazione del canone concordato (evidenziate per facilità di lettura nelle Figura 30, Figura 32 e Figura 34); nell' Appendice online invece sono riportati i grafici di dettaglio relativi alle trentadue aree omogenee OMI.

Al loro interno, le linee blu rappresentano i prezzi di mercato al metro quadro, massimi e minimi; quelle tratteggiate in rosso i prezzi a canone concordato; la retta grigia (i cui valori sono sull'asse destro) indica il numero di abitazioni di riferimento per area di applicazione del canone concordato e numero di vani.

\footnotetext{
${ }^{40}$ Si veda la sezione "I vani" nella Nota Metodologica.

${ }^{41}$ Si veda la sezione "I dati" nella Nota Metodologica.
} 
Osservando i grafici occorre innanzitutto sottolineare il fatto che per i monolocali il canone concordato rappresenta un'opzione leggermente più costosa nei suoi valori massimi rispetto all'affitto nel libero mercato. Rimandando all'Appendice per un confronto dettagliato all'interno delle singole zone $\mathrm{OMI}$, possiamo comunque affermare che in media, a parità di superficie, un monolocale costerà leggermente meno con contratti di libero mercato. Ciò è da ricondurre principalmente alla definizione, negli accordi 2008 e 2013 tra inquilini e proprietari, di parametri che tengono conto anche del numero di vani, oltre che della superficie.

I valori di mercato rilevati dall'OMI invece, sono identici per qualsiasi tipologia di abitazione, venendo moltiplicati per la sola superficie: questo è il motivo per cui, all'interno delle varie zone, essi rimangono uguali, risultando via via meno convenienti all'aumentare del numero di stanze e dunque allo scalare del moltiplicatore del canone.

Sebbene sulla carta le abitazioni con sei o più locali risultino essere a canone concordato ampiamente più convenienti che i valori $\mathrm{OMI}$, come verrà riscontrato più avanti nella discussione, esse appaiono essere meno presenti nelle zone di maggior interesse (economico) per le famiglie, surrogando l'idea che la struttura del mercato cittadino tenda ad escludere i nuclei familiari dalla locazione.

Valutando l'andamento dei prezzi di mercato, da un lato si osserva il progressivo calo dei prezzi unitari via via che ci si allontana dalla "Zona di Pregio", dall'altro lo scarto tra i valori di inizio e fine periodo è più marcato nelle zone più costose. Si segnala infine una decrescita più evidente nei valori massimi piuttosto che nei valori minimi, mentre il biennio 2013-2015 appare essere quello che presenta la principale fase di discesa dei prezzi, intervallando due periodi di maggiore stabilità. 
Figura 30. Collocazione zona di applicazione del canone concordato "Zona di pregio".

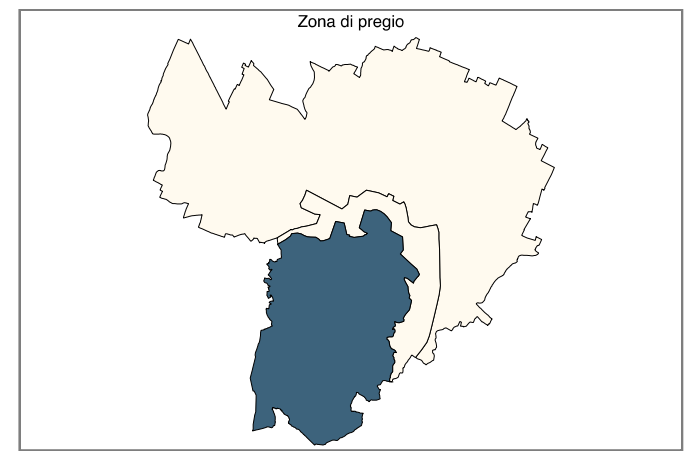

Figura 31. Andamento dei valori al MQ a canone di mercato e canone concordato per numero di vani, nella "Zona di pregio" (anni 2009-2017). N totale abitazioni per tipologia.

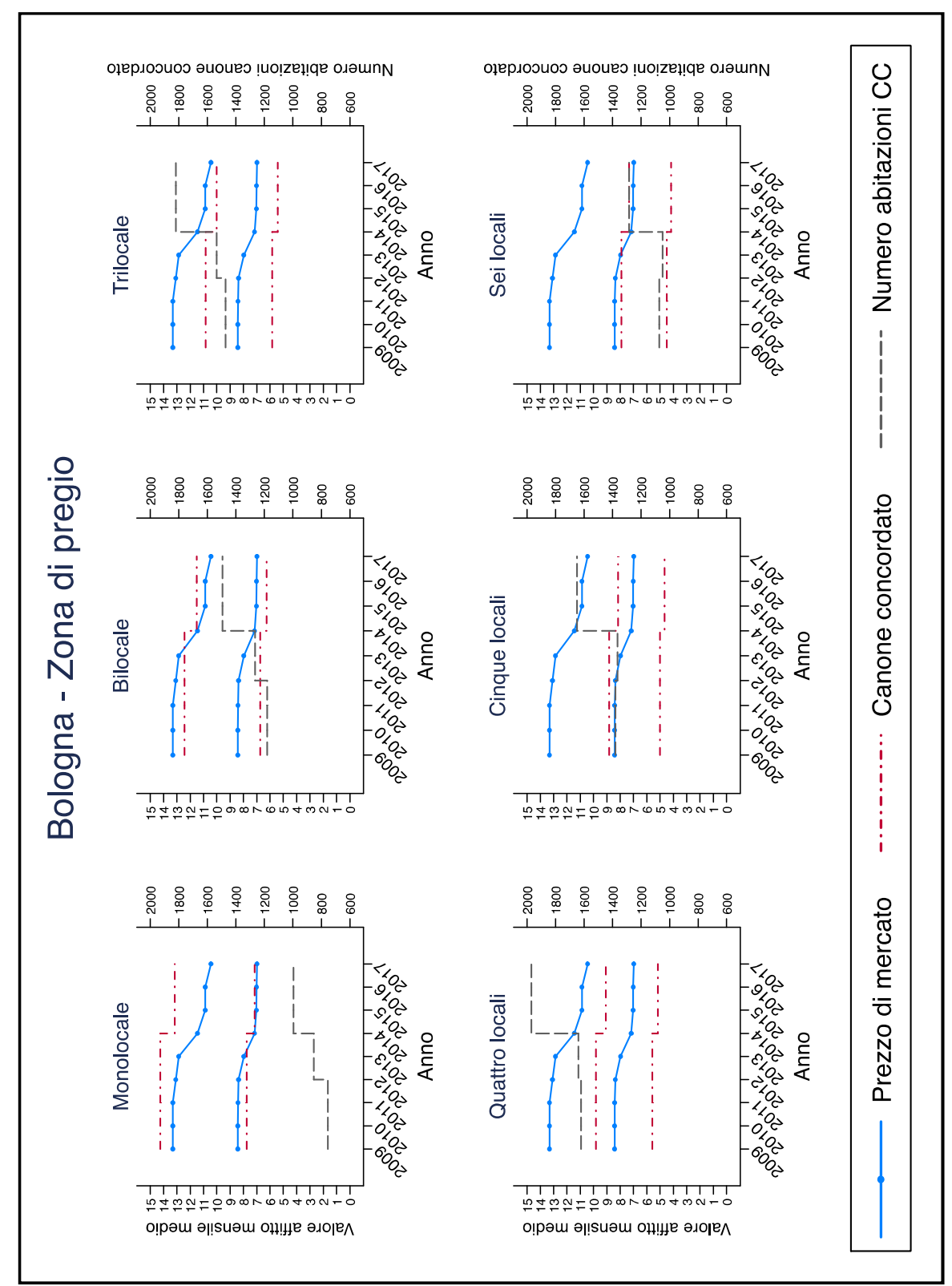


Figura 32. Collocazione zona di applicazione del canone concordato "Zona A".

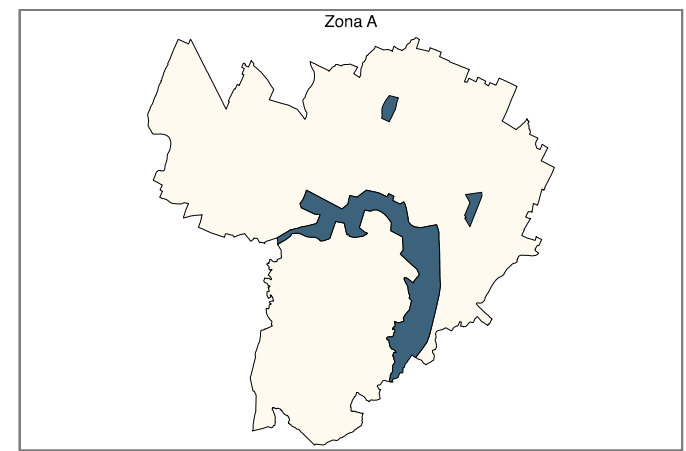

Figura 33. Andamento dei valori al MQ a canone di mercato e canone concordato per numero di vani, nella "Zona A" (anni 2009-2017). N totale abitazioni per tipologia.

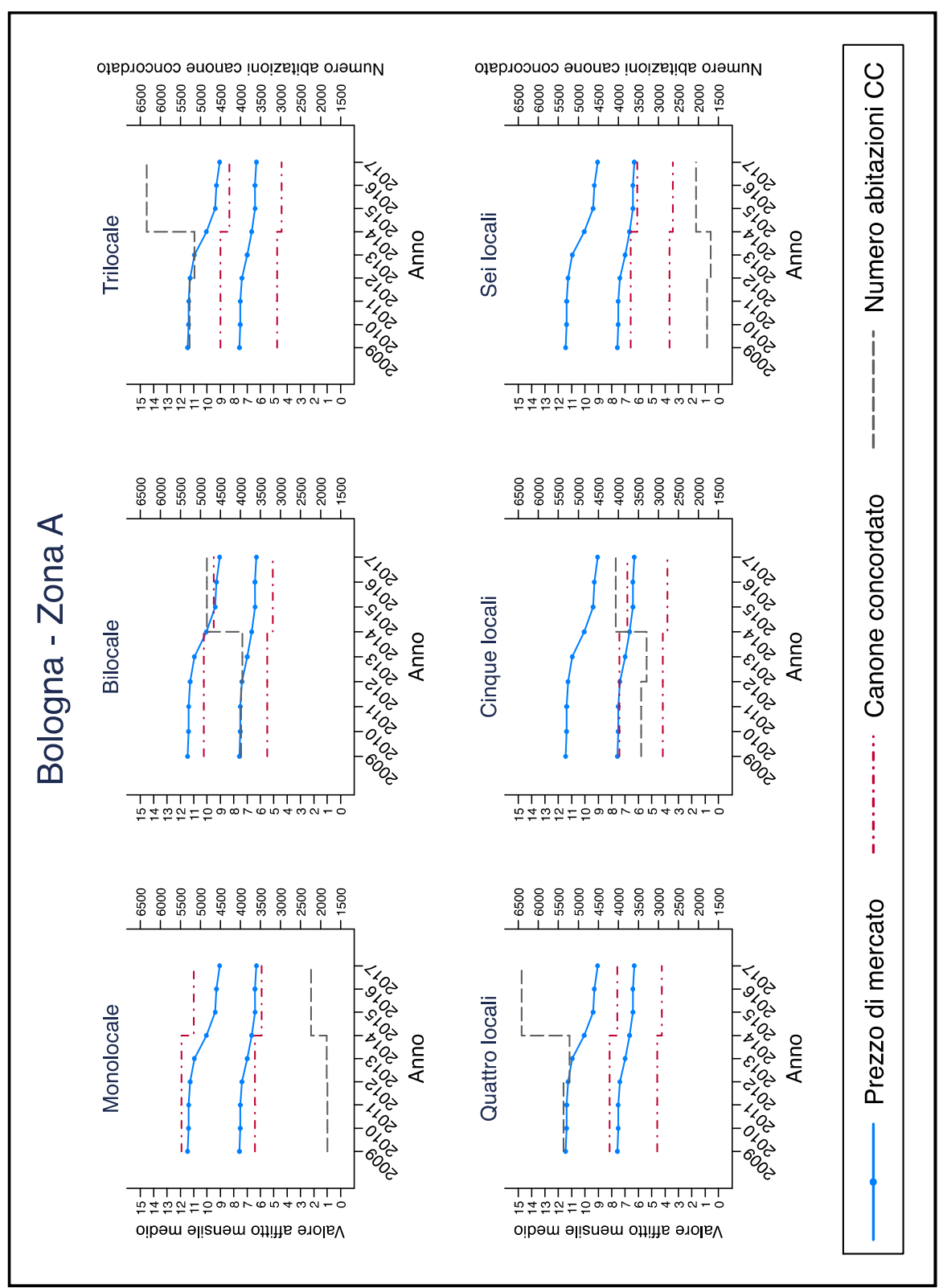


Figura 34. Collocazione zona di applicazione del canone concordato "Zona B".

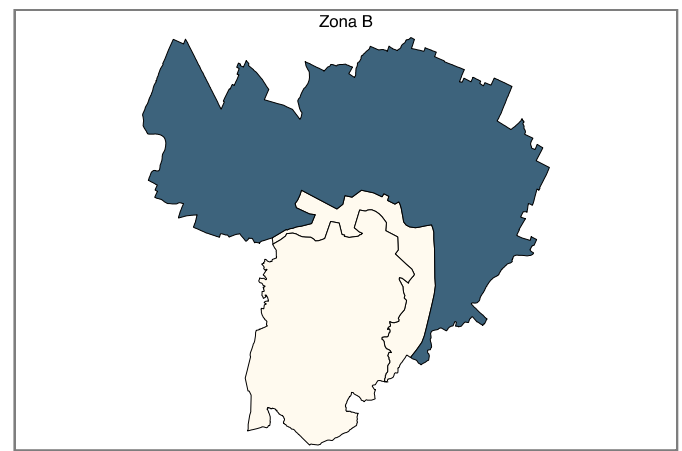

Figura 35. Andamento dei valori al MQ a canone di mercato e canone concordato per numero di vani, nella "Zona B" (anni 2009-2017). N totale abitazioni per tipologia.

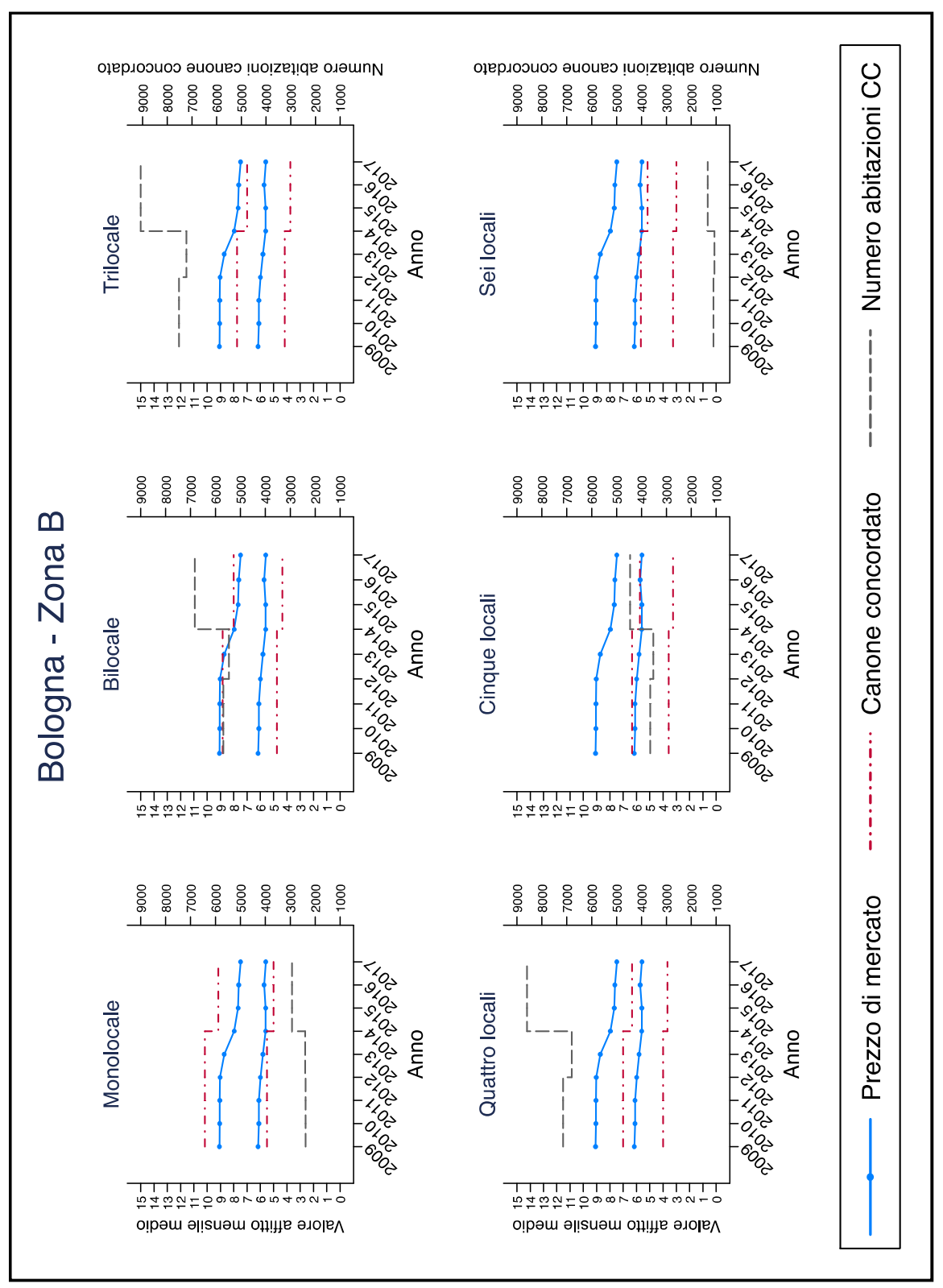




\section{Gli affitti mensili a Bologna e nelle sue zone}

\section{La definizione del numero di vani}

L'analisi dei prezzi al metro quadro non permette tuttavia di comprendere gli effetti di tali variazioni di prezzo sulla condizione delle famiglie né, in generale, di comprendere come sia strutturata l'offerta locativa a Bologna. Per completare il quadro del mercato è necessario estendere l'analisi agli affitti mensili lordi e netti per abitazione. A tal fine occorre riprendere la definizione del numero di vani per le abitazioni incluse nella nostra popolazione di riferimento, verificando poi, conseguentemente, le superfici medie degli alloggi nelle diverse zone della città. Ci si sofferma quindi sui prezzi degli affitti potenziali a canone concordato e sul libero mercato, confrontandoli, per poi introdurre, sul finire del capitolo, il concetto di sostenibilità economica per le famiglie mediane bolognesi.

La definizione del numero dei vani risulta fondamentale per stimare il valore dell'affitto mensile a canone concordato delle abitazioni incluse negli stock di immobili (2010, 2012 e 2014) che fungono da popolazione di riferimento di questa analisi ${ }^{42}$. È dunque opportuno riproporre qui a quali fasce di superficie corrispondano le sei tipologie di abitazione per numero di vani, ribadendo che si tratta di una definizione non mutuamente esclusiva, costruita a partire dalle informazioni rilevate a questo proposito dal Censimento Istat sugli immobili del 2011 (si veda la Tabella 29).

Tabella 29 - Distribuzione percentuale dei vani per classi di superficie.

\begin{tabular}{|c|c|c|c|c|c|c|c|}
\hline & \multicolumn{7}{|c|}{ Numero di vani } \\
\hline Classi di superficie & 1 & 2 & 3 & 4 & 5 & 6 & Totale \\
\hline fino a $29 \mathrm{mq}$ & 3.8 & 0.7 & 0.2 & & & & 0.7 \\
\hline $30-39 \mathrm{mq}$ & 31.7 & 10.6 & 1.4 & 0.2 & & & 10.6 \\
\hline $40-49 \mathrm{mq}$ & 33.2 & 24.9 & 5.8 & 1.2 & 0.6 & & 24.9 \\
\hline $50-59 \mathrm{mq}$ & 15.7 & 22.5 & 15.4 & 3.0 & 1.3 & 0.5 & 22.5 \\
\hline $60-79 \mathrm{mq}$ & 8.5 & 30.3 & 52.5 & 30.2 & 7.1 & 3.6 & 30.3 \\
\hline $80-99 \mathrm{mq}$ & 3.5 & 6.9 & 17.6 & 40.8 & 27.7 & 8.5 & 6.9 \\
\hline $100-119 \mathrm{mq}$ & 1.4 & 1.8 & 4.1 & 17.0 & 31.9 & 13.3 & 1.8 \\
\hline $120-149 \mathrm{mq}$ & 2.0 & 1.2 & 1.3 & 5.1 & 22.7 & 23.4 & 1.2 \\
\hline 150 e più mq & 0.1 & 1.2 & 1.7 & 2.6 & 8.6 & 50.8 & 1.2 \\
\hline Totale (N) & 8682 & 27174 & 53107 & 51945 & 26131 & 15638 & 182677 \\
\hline
\end{tabular}

A partire dalle distribuzioni di frequenza appena presentate, per ciascuna tipologia di abitazione si considerano le classi di superficie che coprono l'80\% intorno alla mediana (segnalate in grassetto nella Tabella 29), stabilendo i seguenti assunti:

${ }^{42}$ Si veda la sezione "I vani” nella Nota Metodologica. 
- Si considerano "monolocali" gli immobili aventi superficie compresa tra 30 e 59 metri quadri;

- Si considerano "bilocali" gli immobili tra 40 e 79 metri quadri;

- Si considerano "trilocali" gli immobili tra 50 e 99 metri quadri;

- Si considerano "quadrilocali" gli immobili aventi superficie compresa tra 60 e 119 metri quadri;

- Si considerano dotati di cinque locali gli immobili tra 80 e 149 metri quadri;

- Si considerano dotati di sei locali gli immobili aventi superficie superiore a 100 metri quadri.

La Tabella 30 riporta invece un prospetto delle superfici medie dei nostri stock di edifici ad uso abitativo per numero di vani.

Tabella 30. Superfici medie $\left(\mathrm{m}^{2}\right)$ delle abitazioni per numero vani. Stock 2010, 2012, 2014.

\begin{tabular}{|l|c|c|c|}
\hline & 2010 & 2012 & 2014 \\
\hline Monolocali & 48.1 & 48.1 & 48.0 \\
\hline Bilocali & 63.1 & 62.7 & 62.9 \\
\hline Trilocali & 74.2 & 74.0 & 74.1 \\
\hline Quadrilocali & 82.6 & 82.5 & 82.7 \\
\hline Cinque locali & 99.4 & 99.3 & 99.3 \\
\hline Sei locali & 128.1 & 127.7 & 127.1 \\
\hline
\end{tabular}

Si può notare una sostanziale stabilità tra gli stock, in termini di superfici medie delle abitazioni, che non può essere ricondotta soltanto alla sovrapposizione delle popolazioni di riferimento, dal momento che tra uno stock e il successivo cambiano rispettivamente 4'532 e 6'198 alloggi (a totali quasi invariati nel primo intervallo, con l'incremento del $23 \%$ dello stock nel secondo). Una spiegazione più convincente risiede probabilmente nella comune tipologia costruttiva degli edifici all'interno delle specifiche zone ${ }^{43}$.

La Figura 36 rappresenta, attraverso tre mappe (relative ai tre stock di abitazioni locate a canone concordato utilizzati), la superficie media delle abitazioni per zona OMI. Ancor più delle tabelle precedenti, rispetto alle quali, tuttavia, viene meno la suddivisione per numero di vani, essa può aiutare ad evidenziare le differenze in termini di metrature nel patrimonio immobiliare comunale.

Innanzitutto, osservando le colorazioni delle zone rimanere immutate nei tre anni di nostro interesse, si ha un'ulteriore riprova della sostanziale invariabilità del dato nel tempo (ad assumere una sfumatura più chiara è la sola zona D16 "Pedecollinare", che cambia la classe di riferimento nella nostra classificazione, pur a fronte di un calo unitario). Grande variabilità, al contrario, vige tra le differenti aree del territorio cittadino. Le metrature maggiori si riscontrano sui colli (Zona R4, $121 \mathrm{~m}^{2}$ medi nel 2014) ed in loro prossimità (Zona D16 "Pedecollinare" e D17 "Pedecollinare e

${ }^{43}$ Si veda la sezione "Le abitazioni a canone concordato" nel secondo capitolo. 
semicollinare"), oltre che nei pressi del centro storico (Zona C11 "Quartiere Marconi" e D23 "Filopanti-Massarenti").

Figura 36. Superficie media delle abitazioni nelle zone omogenee OMI, con riferimento agli stock 2010, 2012 e 2014.

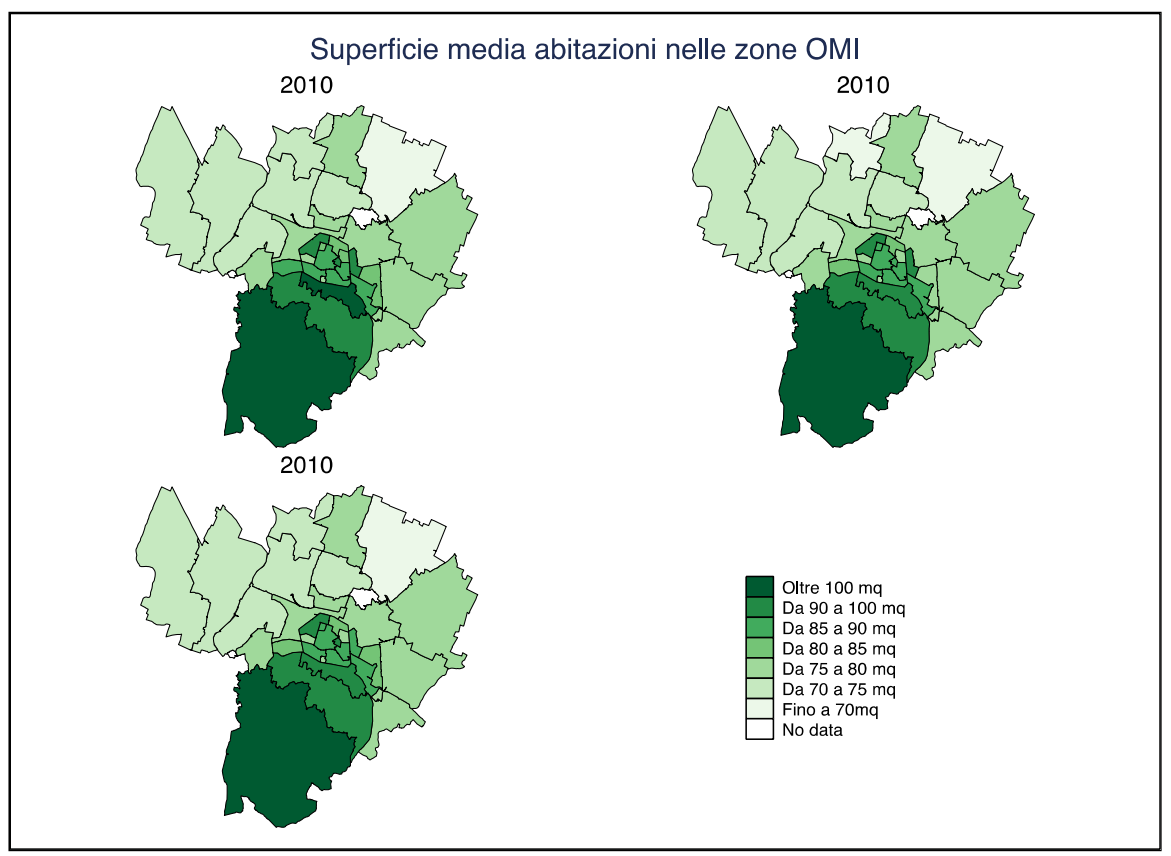

Di contro, superfici decisamente inferiori alla media si riscontrano nelle aree rurali R1, R2 e R3, ai margini della città (è infatti l'area "Agricola Nord-Est" a registrare le metrature più basse, $67 \mathrm{~m}^{2}$ medi nel 2014), e in quartieri popolari come Barca (D20) e Bolognina-Arcoveggio (D8), dove ci si attesta leggermente al di sopra dei $70 \mathrm{~m}^{2}$.

\section{Gli affitti potenziali a canone concordato}

La Tabella 31 raffigura i valori potenziali degli affitti mensili a canone concordato, nei tre anni del periodo di interesse della nostra analisi.

Per ovviare alle inevitabili influenze sulla media che risultano dalla definizione non univoca dei vani, il procedimento per la determinazione dei valori minimi e massimi riportati in tabella è il seguente: i parametri minimi e massimi stabiliti dagli accordi di canone a seconda della dotazione di elementi di pregio vengono moltiplicati per la superficie di ogni abitazione; viene poi elaborata una media tra i due risultati così conseguiti. Infine, osservando la distribuzione dei valori medi degli affitti zona per 
zona, si definiscono due soglie relative alle medie dei valori del primo e del quarto quartile della distribuzione ${ }^{44}$.

Tabella 31. Affitti medi potenziali a canone concordato, per zona di applicazione del canone e numero di vani.

Anni 2010, 2012, 2014.

\begin{tabular}{|c|c|c|c|c|c|c|c|}
\hline \multirow{2}{*}{ Zone } & \multirow{2}{*}{ Vani } & \multicolumn{2}{|c|}{2010} & \multicolumn{2}{|c|}{2012} & \multicolumn{2}{|c|}{2014} \\
\hline & & $\operatorname{Min}(€)$ & $\operatorname{Max}(€)$ & $\operatorname{Min}(€)$ & $\operatorname{Max}(€)$ & $\operatorname{Min}(€)$ & $\operatorname{Max}(€)$ \\
\hline \multirow{6}{*}{ 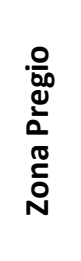 } & 1 & 388 & 623 & 386 & 625 & 356 & 576 \\
\hline & 2 & 438 & 727 & 434 & 724 & 402 & 671 \\
\hline & 3 & 470 & 773 & 468 & 772 & 433 & 716 \\
\hline & 4 & 521 & 847 & 518 & 843 & 479 & 783 \\
\hline & 5 & 592 & 928 & 590 & 923 & 549 & 856 \\
\hline & 6 & 654 & 1210 & 654 & 1212 & 608 & 1125 \\
\hline \multirow{6}{*}{$\begin{array}{l}\varangle \\
\text { O } \\
\text { ป } \\
\text { N }\end{array}$} & 1 & 339 & 527 & 337 & 526 & 313 & 486 \\
\hline & 2 & 377 & 596 & 376 & 595 & 349 & 551 \\
\hline & 3 & 395 & 631 & 394 & 630 & 365 & 584 \\
\hline & 4 & 417 & 670 & 417 & 669 & 388 & 622 \\
\hline & 5 & 484 & 730 & 485 & 728 & 446 & 670 \\
\hline & 6 & 529 & 870 & 529 & 863 & 490 & 783 \\
\hline \multirow{6}{*}{$\begin{array}{l}\infty \\
\text { D } \\
\text { D } \\
\text { N }\end{array}$} & 1 & 293 & 453 & 293 & 452 & 267 & 409 \\
\hline & 2 & 331 & 516 & 329 & 515 & 300 & 468 \\
\hline & 3 & 345 & 539 & 343 & 538 & 310 & 486 \\
\hline & 4 & 360 & 550 & 359 & 551 & 327 & 501 \\
\hline & 5 & 406 & 564 & 406 & 566 & 368 & 512 \\
\hline & 6 & 452 & 684 & 453 & 669 & 414 & 611 \\
\hline
\end{tabular}

Per ciò che concerne gli anni inclusi nella tabella, la scelta è ricaduta su 2010, 2012 e 2014 non soltanto in quanto corrispondenti ai tre stock di abitazioni che costituiscono la popolazione di riferimento della ricerca, ma anche perché si intersecano con i due differenti accordi di canone del 2008 e 2013, entrati rispettivamente in vigore nel 2009 e nel 2014. Dal momento che a determinare gli affitti medi potenziali concorrono i parametri del canone concordato e le superfici delle singole abitazioni, questi tre momenti concentrano tutte le variazioni intervenute nell'arco temporale di nostro interesse.

Si riscontra in maniera evidente l'abbassamento dei prezzi intervenuto dall'accordo 2013, di cui già si era avuta testimonianza osservando l'andamento dei valori al metro quadro. Ad esso è quasi unicamente dovuto il calo degli affitti mensili potenziali, in ragione della sostanziale uniformità delle metrature medie delle abitazioni cui si accennava in precedenza.

\footnotetext{
${ }^{44}$ Si veda la sezione "Il calcolo dei prezzi a canone concordato" nella Nota Metodologica.
} 
Inoltre, l'aumento del numero dei vani e delle superfici corrispondenti fa lievitare i valori degli affitti potenziali. La presenza di un rapporto inversamente proporzionale con cui i prezzi al metro quadro calano all'aumentare dei locali, ${ }^{45}$ permette di evitare l'esplosione degli affitti degli alloggi di maggiore ampiezza (ovvero, come nella realtà autonomamente regolata dai valori di mercato, le abitazioni a maggiore metratura hanno una domanda di mercato inferiore, dato il loro costo e quindi il valore marginale per ogni metro in più è decrescente).

\section{Gli affitti potenziali a canone di mercato}

Per ciò che concerne l'analisi dei valori potenziali minimi e massimi degli affitti a canone libero nelle trentadue aree omogenee, non ci si addentra in questa sede nel dettaglio di ogni specifica zona e tipologia di abitazione, rimandando all'Appendice online per le tabelle che presentano tali dati. Ci limiteremo al momento a citare alcune chiavi di lettura comuni relativamente all'andamento dei prezzi che definiamo " $\mathrm{di}$ mercato" ma che, ricordiamo, in ragione della metodologia applicata da OMI, risentono del numero consistente di contratti a canone concordato siglati. A questo fine, sono proposte le Tabella 32 e Tabella 33, che riportano l'andamento nel periodo considerato (2009-2017) degli affitti potenziali, minimi e massimi, nelle zone $\mathrm{OMI}^{46}$ Al loro interno, giova specificarlo, sono riportati prezzi calcolati sulla base delle dimensioni del singolo alloggio, indipendentemente dunque dal numero di vani.

In primis, sull'intero periodo si conferma la tendenza al calo dei prezzi già emersa discutendo i valori al metro quadro: il decremento si attesta attorno al 20\%, con lievi scarti decimali tra massimi e minimi e tra le varie tipologie di abitazione.

Possiamo confermare una caduta degli affitti potenziali superiore alla media nelle zone C11 "Quartiere Marconi"; C3 "Irnerio - Centotrecento - Moline - Righi - P.zza VIII Agosto - Indipendenza - V.li Masini e Pichat"); D16 ("Pedecollinare"); D21 ("Andrea Costa - Saragozza"); D22 (“Corticella"); D23 ("V.le Filopanti - Massarenti Zanolini - Stazione S. Vitale").

Appare rilevante sottolineare che un'abitazione con sei locali, a canone concordato, richiede un esborso mensile doppio rispetto ad un monolocale nella medesima zona; a canone di mercato l'affitto tende a triplicare o più.

\footnotetext{
${ }^{45} \mathrm{Si}$ veda la sezione "I valori mensili a metro quadro a canone concordato" nel capitolo relativo ai prezzi al metro quadro.

${ }^{46} \mathrm{Gli}$ affitti mensili sono calcolati sulla base della superficie di ogni abitazione presente nella zona OMI considerata: si consideri dunque che le differenze di prezzo tra le zone sono influenzate, tra altri fattori, anche dalla dimensione degli alloggi in esse presenti.
} 
Tabella 32. Andamento affitti potenziali minimi mensili, nelle aree omogenee OMI. Prezzi minimi 2009 e 2017 (espressi in €), variazioni \% sull'anno precedente e sul periodo.

\begin{tabular}{|c|c|c|c|c|c|c|c|c|c|c|c|}
\hline \multicolumn{12}{|c|}{ Andamento prezzi mensili minimi } \\
\hline \multirow{2}{*}{$\begin{array}{l}\text { Zona } \\
\text { OMI }\end{array}$} & \multirow{2}{*}{$\begin{array}{c}\text { MIN (€) } \\
2009\end{array}$} & \multicolumn{8}{|c|}{ Variazioni (\%) sull'anno precedente } & \multirow{2}{*}{$\begin{array}{c}\text { MIN (€) } \\
2017\end{array}$} & \multirow{2}{*}{$\begin{array}{c}\Delta 2017- \\
2009 \\
(\%)\end{array}$} \\
\hline & & 2010 & 2011 & 2012 & 2013 & 2014 & 2015 & 2016 & 2017 & & \\
\hline B1 & 784 & - & -0.4 & -2.2 & -7.9 & -15.4 & - & - & - & 595 & -24 \\
\hline B7 & 798 & - & - & -4.8 & -5.9 & -3.5 & - & - & -1.0 & 621 & -22 \\
\hline C10 & 719 & -11.8 & - & 2.0 & -6.7 & -11.2 & 19.0 & - & -6.7 & 596 & -17 \\
\hline C11 & 876 & -5.3 & - & -5.4 & -10.4 & -4.9 & -15.0 & - & -4.0 & 546 & -38 \\
\hline C3 & 800 & - & - & -3.7 & -3.7 & -12.0 & -6.3 & - & -6.7 & 572 & -29 \\
\hline C4 & 710 & - & - & -10.2 & -6.1 & -9.8 & - & - & - & 540 & -24 \\
\hline C5 & 786 & - & - & -10.5 & -4.8 & -3.7 & - & - & -3.4 & 623 & -21 \\
\hline C6 & 748 & - & - & -0.2 & -2.4 & -9.9 & - & - & - & 656 & -12 \\
\hline C7 & 636 & - & - & -0.5 & -3.1 & -5.5 & - & - & - & 579 & -9 \\
\hline C8 & 738 & - & - & -5.0 & -8.1 & -7.1 & -5.1 & -1.5 & - & 559 & -24 \\
\hline C9 & 663 & - & - & -2.0 & -4.1 & -7.7 & - & - & - & 575 & -13 \\
\hline D16 & 852 & - & - & -0.7 & - & -12.4 & -6.7 & - & - & 691 & -19 \\
\hline D17 & 716 & - & - & - & -3.8 & -2.5 & -4.8 & - & - & 639 & -11 \\
\hline D19 & 550 & - & - & -1.1 & -2.8 & -2.0 & - & - & - & 518 & -6 \\
\hline D2 & 544 & - & - & -4.4 & -2.3 & -3.5 & - & - & - & 490 & -10 \\
\hline D20 & 430 & - & - & -0.2 & -1.7 & -8.5 & - & 13.0 & -15.6 & 369 & -14 \\
\hline D21 & 698 & - & - & -2.2 & -10.2 & -14.3 & - & - & - & 526 & -25 \\
\hline D22 & 459 & - & - & -0.9 & - & -2.8 & -19.5 & - & - & 357 & -22 \\
\hline D23 & 677 & - & - & -1.4 & -3.4 & -6.7 & -21.6 & - & - & 471 & -30 \\
\hline D24 & 642 & - & - & -1.2 & -5.4 & 12.5 & -6.5 & - & - & 631 & -2 \\
\hline D25 & 493 & - & - & - & -4.6 & -9.6 & - & -4.5 & - & 406 & -18 \\
\hline D3 & 529 & - & - & -2.4 & -6.0 & -4.4 & -1.7 & - & - & 456 & -14 \\
\hline D4 & 519 & - & - & -3.5 & -2.3 & -7.2 & - & - & - & 454 & -12 \\
\hline D5 & 488 & - & - & -1.5 & -4.7 & 0.2 & - & - & - & 459 & -6 \\
\hline D7 & 474 & -3.2 & - & -2.1 & - & -1.2 & -8.7 & - & - & 405 & -14 \\
\hline D8 & 435 & -4.2 & - & -4.5 & -3.6 & -1.4 & - & 3.8 & 1.9 & 400 & -8 \\
\hline D9 & 429 & -2.6 & - & -5.3 & -13.6 & -1.5 & 12.9 & -1.9 & - & 372 & -13 \\
\hline E1 & 487 & -1.6 & - & -2.5 & -1.6 & 0.5 & nd & nd & nd & nd & nd \\
\hline R1 & 342 & - & - & -2.2 & -1.1 & 7.9 & nd & nd & nd & nd & nd \\
\hline R2 & 293 & - & - & -9.8 & - & -1.4 & nd & nd & nd & nd & nd \\
\hline R3 & nd & nd & nd & nd & nd & nd & nd & nd & nd & nd & nd \\
\hline R4 & 896 & - & - & -0.7 & -1.3 & -0.8 & nd & nd & nd & nd & nd \\
\hline
\end{tabular}

Quella appena sottolineata risulta essere una dinamica estremamente rilevante ai fini di un confronto tra le due forme contrattuali, sia che la si osservi dal punto di vista del locatore che dal punto di vista dell'affittuario. 
Tabella 33. Andamento affitti potenziali massimi mensili, nelle aree omogenee OMI. Prezzi massimi 2009 e 2017 (espressi in €), variazioni \% sull'anno precedente e sul periodo.

\begin{tabular}{|c|c|c|c|c|c|c|c|c|c|c|c|}
\hline \multicolumn{12}{|c|}{ Andamento prezzi mensili massimi } \\
\hline \multirow{2}{*}{$\begin{array}{l}\text { Zona } \\
\text { OMI }\end{array}$} & \multirow{2}{*}{$\begin{array}{c}\operatorname{MAX}(€) \\
2009\end{array}$} & \multicolumn{8}{|c|}{ Variazioni (\%) sull’anno precedente } & \multirow{2}{*}{$\begin{array}{c}\operatorname{MAX}(€) \\
2017\end{array}$} & \multirow{2}{*}{$\begin{array}{c}\Delta 2017- \\
2009 \\
(\%)\end{array}$} \\
\hline & & 2010 & 2011 & 2012 & 2013 & 2014 & 2015 & 2016 & 2017 & & \\
\hline B1 & 1217 & - & - & -2.0 & -2.4 & -9.2 & - & - & -12.2 & 927 & -24 \\
\hline B7 & 1126 & - & - & -6.0 & - & 4.3 & - & - & -20.8 & 874 & -22 \\
\hline C10 & 1016 & -4.2 & - & 2.0 & - & -16.4 & 2.6 & 10.0 & -18.2 & 767 & -25 \\
\hline C11 & 1175 & -5.9 & - & -3.6 & - & -14.7 & -12.5 & - & -8.6 & 728 & -38 \\
\hline C3 & 1095 & - & - & -3.7 & - & -16.8 & -14.0 & - & -8.1 & 694 & -37 \\
\hline C4 & 973 & - & - & -4.7 & - & -16.7 & -2.5 & - & - & 753 & -23 \\
\hline C5 & 1077 & - & - & -5.1 & - & -8.4 & - & - & 2.3 & 958 & -11 \\
\hline C6 & 1275 & - & - & -0.2 & - & -8.8 & -6.8 & - & - & 1081 & -15 \\
\hline C7 & 954 & - & - & -0.5 & - & -15.5 & - & - & - & 802 & -16 \\
\hline C8 & 1073 & - & - & -2.0 & -4.2 & -9.0 & -9.5 & - & - & 830 & -23 \\
\hline C9 & 1014 & - & - & -2.0 & - & -11.2 & -8.7 & - & - & 805 & -21 \\
\hline D16 & 1353 & - & - & -6.2 & - & -22.1 & -12.5 & - & - & 864 & -36 \\
\hline D17 & 1271 & - & - & -6.7 & -3.8 & -3.9 & -2.1 & - & - & 1073 & -16 \\
\hline D19 & 884 & - & - & -5.1 & -2.3 & -2.8 & -17.5 & -1.2 & - & 649 & -27 \\
\hline D2 & 846 & - & - & -0.8 & -4.8 & -8.6 & -1.6 & - & - & 719 & -15 \\
\hline D20 & 622 & - & -0.2 & -0.4 & - & -17.8 & - & 1.4 & -13.2 & 447 & -28 \\
\hline D21 & 1053 & - & - & -1.6 & -6.1 & -13.6 & -10.0 & - & -6.7 & 706 & -33 \\
\hline D22 & 708 & - & - & 0.8 & -5.4 & -12.2 & -17.7 & - & - & 488 & -31 \\
\hline D23 & 1120 & - & - & -1.4 & - & -6.6 & -28.3 & -6.1 & - & 696 & -38 \\
\hline D24 & 1074 & - & -0.3 & 0.3 & -2.9 & 12.8 & -5.6 & - & - & 1110 & 3 \\
\hline D25 & 758 & - & -0.5 & - & -5.8 & -11.9 & - & -10.3 & - & 562 & -26 \\
\hline D3 & 764 & - & - & -0.9 & -5.1 & -9.6 & -3.0 & - & - & 629 & -18 \\
\hline D4 & 692 & - & - & -0.5 & -2.8 & -2.2 & -5.9 & - & - & 616 & -11 \\
\hline D5 & 733 & - & - & 0.1 & -3.6 & -9.4 & -1.8 & -2.4 & - & 614 & -16 \\
\hline D7 & 815 & - & - & 0.4 & -2.3 & -13.1 & -19.4 & - & - & 560 & -31 \\
\hline D8 & 616 & - & - & -0.1 & -5.9 & -7.6 & - & 2.0 & - & 546 & -11 \\
\hline D9 & 671 & -5.6 & - & -3.6 & -11.8 & -8.8 & 10.3 & -3.3 & - & 524 & -22 \\
\hline E1 & 682 & -2.3 & -0.3 & -2.1 & -1.5 & -5.1 & - & - & - & nd & nd \\
\hline R1 & 564 & - & -0.7 & -1.1 & -1.3 & -2.0 & nd & nd & nd & nd & nd \\
\hline R2 & 513 & - & - & -9.8 & - & -5.6 & nd & nd & nd & nd & nd \\
\hline R3 & nd & nd & nd & nd & nd & nd & nd & nd & nd & nd & nd \\
\hline R4 & 1433 & - & - & -0.7 & - & -16.6 & nd & nd & nd & nd & nd \\
\hline
\end{tabular}

Essa, inoltre, costituisce un ulteriore indizio nell'indicare gli alloggi di dimensione e struttura più adeguata all'uso familiare (le abitazioni a metratura superiore agli $80 \mathrm{mq}$.) come meno disponibili sul mercato a canone concordato e, anche laddove 
disponibili, come vedremo, spesso fuori dalla portata economica delle famiglie stesse. Questo aspetto appare centrale ai fini della ricerca svolta e, in generale, risulta essere il cuore della problematica per l'amministrazione, sia del Comune di Bologna che della Città Metropolitana.

Per concludere questa sezione, la Tabella $34^{47}$ individua le cinque zone OMI più costose e le cinque zone OMI meno costose per tipologia di abitazione, in termini di affitto medio mensile a canone di mercato. Agli estremi della graduatoria, salvo rare eccezioni, individuiamo con costanza le medesime zone. Ai vertici, la zona D24 "Murri", la zona C6 entro cui ricade il quartiere Galvani - D'Azeglio - Santo Stefano, la D17 "Pedecollinare". A queste, sempre presenti, si affiancano altre zone ricorrenti, tra cui la zona C5 "San Vitale - Aldrovandi - Guerrazzi - Santo Stefano", la zona C9 entro cui si colloca il quartiere Malpighi - San Felice, la zona B1 "Centro Storico". In breve, dunque, a monopolizzare il vertice della graduatoria di prezzo degli affitti sono settori della città che ricadono entro la cerchia dei viali, o appena a sud di essa.

Tabella 34. Prospetto delle cinque zone OMI più costose e delle cinque zone OMI meno costose per tipologia di abitazione. Affitti mensili medi a canone di mercato (€).

\begin{tabular}{|c|c|c|c|c|c|c|c|c|c|c|c|c|}
\hline \multirow{2}{*}{2017} & \multicolumn{2}{|c|}{ Monolocali } & \multicolumn{2}{|c|}{ Bilocali } & \multicolumn{2}{|c|}{ Trilocali } & \multicolumn{2}{|c|}{ Quadrilocali } & \multicolumn{2}{|c|}{$\begin{array}{l}\text { Cinque } \\
\text { locali }\end{array}$} & \multicolumn{2}{|c|}{ Sei locali } \\
\hline & Zona & $€$ & Zona & $€$ & Zona & $€$ & Zona & $€$ & Zona & $€$ & Zona & $€$ \\
\hline \multirow{5}{*}{ 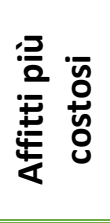 } & D24 & 508 & D24 & 655 & $\mathrm{D} 24$ & 762 & C6 & 849 & C6 & 1079 & C6 & 1394 \\
\hline & C6 & 460 & $\mathrm{C} 6$ & 595 & $\mathrm{C} 6$ & 715 & D24 & 845 & D24 & 1008 & $\mathrm{C} 5$ & 1300 \\
\hline & D17 & 442 & D17 & 579 & D17 & 700 & D17 & 814 & D17 & 990 & D17 & 1284 \\
\hline & C9 & 421 & C9 & 558 & C5 & 670 & C5 & 782 & B1 & 960 & D24 & 1254 \\
\hline & $\mathrm{C7}$ & 418 & $\mathrm{C} 5$ & 539 & C9 & 653 & $\mathrm{~B} 1$ & 757 & C5 & 956 & B1 & 1218 \\
\hline \multirow{5}{*}{ 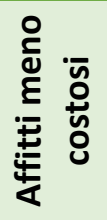 } & D9 & 303 & D7 & 390 & D7 & 468 & D7 & 519 & D7 & 615 & D7 & 776 \\
\hline & D7 & 298 & D25 & 389 & D25 & 465 & D8 & 508 & D8 & 609 & D25 & 761 \\
\hline & D23 & 297 & D9 & 375 & D9 & 454 & D9 & 508 & D9 & 583 & D9 & 716 \\
\hline & D20 & 278 & D22 & 362 & D20 & 415 & D20 & 450 & D22 & 539 & D22 & 681 \\
\hline & D22 & 275 & D20 & 359 & D22 & 410 & D22 & 446 & D20 & 531 & D20 & 649 \\
\hline Media & & 350 & & 453 & & 536 & & 604 & & 745 & & 992 \\
\hline
\end{tabular}

All'estremo opposto, si collocano stabilmente zone più popolari della città: la zona D20 "Santa Viola - Barca", la D22, ossia Corticella; le zone circostanti al quartiere Bolognina e a Piazza dell'Unità (D7, D8); la zona D9 “Marco Polo - Pescarola"; la zona D25 "Viale Filopanti - Via del Chiù".

\footnotetext{
${ }^{47}$ Anche per la definizione degli affitti mensili contenuti in questa tabella è stata applicata una definizione non univoca del numero di vani delle abitazioni.
} 


\section{Canone concordato e libero mercato: I'andamento degli affitti a Bologna.}

Il paragrafo finale di questo capitolo è dedicato ad un confronto tra i due regimi contrattuali all'interno delle tre zone - Zona di Pregio, Zona A e Zona B - in cui gli accordi tra proprietari e inquilini dividono Bologna. Oltre agli affitti potenziali minimi e massimi, a canone concordato e a canone di mercato, la cui procedura di determinazione è illustrata nei precedenti paragrafi dedicati, nei grafici sottostanti si introduce un ulteriore fondamentale elemento, che troverà ampio approfondimento nel capitolo successivo: la sostenibilità.

Per sostenibilità si intende la possibilità per un determinato nucleo familiare di far fronte ai costi di una locazione, e dunque di poter accedere ad un'abitazione. La letteratura definisce nel $30 \%$ del reddito familiare mensile netto il limite massimo oltre il quale un affitto diventa economicamente insostenibile ${ }^{48}$. A tale soglia si perviene scontando il reddito equivalente pro-capite dei cittadini dall'aliquota IRPEF, moltiplicando quanto ottenuto per l'opportuno coefficiente della scala OCSE modificata (a seconda della numerosità della famiglia), dividendo su dodici mensilità il reddito netto familiare che ne risulta e considerandone, appunto, i tre decimi.

Per valutare la sostenibilità delle differenti tipologie di abitazioni, ad ognuna di esse sono associate le soglie di due specifici nuclei:

- La soglia per i nuclei familiari di due persone, in quanto categoria mediana nella distribuzione delle famiglie bolognesi, è posta a confronto con tutti i tipi di immobile.

- La soglia per i nuclei composti da una sola persona è considerata per i monolocali e i bilocali.

- La soglia per i nuclei di tre persone è considerata per trilocali e quadrilocali.

- La soglia per i nuclei di quattro persone è considerata per le abitazioni con cinque o più locali.

Figura 37, Figura 38 e Figura 39 sono riferite alle tre zone di applicazione del canone concordato. Nei grafici in esse contenuti, le linee azzurre continue raffigurano i valori minimi e massimi degli affitti potenziali di mercato; le linee tratteggiate rosse rappresentano graficamente l'equivalente per il canone concordato; le linee verdi tracciano le soglie di sostenibilità per le tipologie di nucleo di volta in volta considerate.

La Tabella 35, invece, presenta lo scarto (espresso in euro) tra prezzi d'affitto a canone concordato e a canone di mercato, per numero di vani e ubicazione. Possiamo affermare che il canone concordato risulta sempre meno costoso per l'inquilino, se si escludono i monolocali (nei loro valori minimi e massimi). Questa tipologia di

\footnotetext{
${ }^{48}$ Per la procedura dettagliata di definizione delle soglie di sostenibilità economica si rimanda alla sezione "La definizione delle soglie di sostenibilità economica" nella Nota Metodologica.
} 
abitazione, infatti, risulta sempre più conveniente a prezzi di mercato, se si esclude la Zona A ad inizio periodo. Nel 2017, infine, diventano convenienti a prezzo di mercato anche i bilocali (nei loro valori minimi) nelle Zone Pregio e A.

Questo primo confronto tra i regimi contrattuali fa emergere due tendenze:

- Al crescere del numero di vani, proprio per effetto dell'applicazione dei medesimi parametri indipendentemente dalle superfici, gli affitti di mercato superano e si distaccano progressivamente da quelli a canone di mercato (per i quali invece si applicava una progressiva riduzione dei valori al metro quadro al crescere dei locali dell'immobile locato).

- I prezzi di mercato fanno registrare cali più rilevanti rispetto a quelli del canone concordato, in particolare nella Zona di Pregio e, in misura leggermente inferiore, nelle restanti fasce. Ciò fa sì che, nel corso degli anni considerati, aumentino le soluzioni a disposizione dell'inquilino più convenienti a canone di mercato.

Tabella 35 - Scarto tra prezzo affitti a canone concordato e a canone di mercato, per zona di applicazione del canone e numero vani. Anni 2009, 2014, 2017. Valori espressi in euro. In grassetto le combinazioni per le quali l'affitto a canone concordato è più alto che a canone di mercato.

Scarto affitto mensile a canone concordato rispetto a canone di mercato - 2009 (€)

\begin{tabular}{l|l|c|c|c|c|c|c|}
\multicolumn{2}{l|}{ Numero vani } & $\mathbf{1}$ & $\mathbf{2}$ & $\mathbf{3}$ & $\mathbf{4}$ & $\mathbf{5}$ & $\mathbf{6 +}$ \\
\hline \multirow{2}{*}{$\begin{array}{l}\text { Prezzi } \\
\text { minimi }\end{array}$} & Zona Pregio & $\mathbf{5}$ & -53 & -139 & -206 & -320 & -477 \\
\cline { 2 - 8 } & Zona A & -5 & -53 & -112 & -154 & -247 & -400 \\
\cline { 2 - 8 } & Zona B & $\mathbf{1 7}$ & -29 & -81 & -118 & -186 & -296 \\
\hline \multirow{2}{*}{$\begin{array}{l}\text { Prezzi } \\
\text { massimi }\end{array}$} & Zona Pregio & $\mathbf{1}$ & -105 & -237 & -355 & -546 & -915 \\
\cline { 2 - 8 } & Zona A & -50 & -154 & -276 & -387 & -566 & -877 \\
\cline { 2 - 8 } & Zona B & $\mathbf{1}$ & -78 & -173 & -241 & -341 & -519 \\
\hline
\end{tabular}

Scarto affitto mensile a canone concordato rispetto a canone di mercato - 2014 (€)

\begin{tabular}{|c|c|c|c|c|c|c|c|}
\hline \multicolumn{2}{|c|}{ Numero vani } & 1 & 2 & 3 & 4 & 5 & $6+$ \\
\hline \multirow{3}{*}{$\begin{array}{l}\text { Prezzi } \\
\text { minimi }\end{array}$} & Zona Pregio & 30 & -19 & -86 & -136 & -221 & -344 \\
\hline & Zona A & 21 & -20 & -70 & -105 & -182 & -311 \\
\hline & Zona B & 21 & -20 & -69 & -102 & -162 & -248 \\
\hline \multirow{3}{*}{$\begin{array}{l}\text { Prezzi } \\
\text { massimi }\end{array}$} & Zona Pregio & 30 & -19 & -86 & -136 & -221 & -344 \\
\hline & Zona A & 21 & -20 & -70 & -105 & -182 & -311 \\
\hline & Zona B & 21 & -20 & -69 & -102 & -162 & -248 \\
\hline
\end{tabular}

Scarto affitto mensile a canone concordato rispetto a canone di mercato - 2017 (€)

\begin{tabular}{|l|l|c|c|c|c|c|c|}
\hline \multicolumn{2}{l|}{ Numero vani } & $\mathbf{1}$ & $\mathbf{2}$ & $\mathbf{3}$ & $\mathbf{4}$ & $\mathbf{5}$ & $\mathbf{6 +}$ \\
\hline \multirow{2}{*}{$\begin{array}{l}\text { Prezzi } \\
\text { minimi }\end{array}$} & Zona Pregio & $\mathbf{5 1}$ & $\mathbf{1 2}$ & -49 & -89 & -157 & -262 \\
\cline { 2 - 9 } & Zona A & $\mathbf{4 7}$ & $\mathbf{1 2}$ & -34 & -68 & -138 & -238 \\
\cline { 2 - 9 } & Zona B & $\mathbf{2 8}$ & -10 & -57 & -87 & -148 & -219 \\
\hline \multirow{2}{*}{$\begin{array}{l}\text { Prezzi } \\
\text { massimi }\end{array}$} & Zona Pregio & $\mathbf{6 4}$ & -11 & -104 & -191 & -337 & -588 \\
\cline { 2 - 9 } & Zona A & $\mathbf{1 1}$ & -80 & -176 & -244 & -379 & -596 \\
\cline { 2 - 9 } & Zona B & $\mathbf{2 1}$ & -45 & -123 & -173 & -253 & -371 \\
\hline
\end{tabular}


Soffermiamoci ora sull'andamento delle soglie di sostenibilità: dal 2009 al 2017 il limite massimo pari al $30 \%$ del reddito netto familiare sale per i nuclei di una ( $+7 \%$ sul periodo) e due persone (+9\% sul periodo); cala invece del $3 \%$ il tetto per le famiglie di tre o quattro membri, pur in presenza di un'inversione di tendenza dal 2014.

Rimandando al successivo capitolo una più approfondita discussione della sostenibilità economica degli affitti nelle aree omogenee OMI (definita rispetto l'affitto potenziale medio a canone concordato, prima ancora del corrispettivo nel libero mercato), alcune osservazioni sono necessarie:

- Nella Zona B gli affitti a canone concordato sono sempre sostenibili per i nuclei da due a quattro persone (le soglie di sostenibilità giacciono sempre al di sopra dei valori massimi di affitto a canone concordato, fatto salvo per i nuclei di due persone in abitazioni di sei locali);

- Nella Zona A i valori massimi degli affitti a canone concordato sono sempre al di sotto delle soglie per le famiglie con tre o più componenti (salvo una leggera sovrapposizione a inizio periodo per gli immobili con sei o più locali); per le famiglie composte da due membri, il canone è sempre sostenibile, anche nei suoi valori massimi, solo per monolocali e bilocali (cui si aggiungono trilocali e quadrilocali dal 2014).

- Nella Zona di Pregio i costi degli affitti sono nettamente superiori; ne consegue che le soglie di sostenibilità siano pressoché sempre comprese tra gli affitti minimi e massimi a canone concordato. Soltanto il calo dei prezzi degli ultimi tre anni fa sì che i nuclei da due persone nei monolocali e quelli da tre nei trilocali registrino tetti al di sopra dei valori massimi di canone.

- Un particolare approfondimento merita la situazione dei nuclei monopersonali: un affitto in Zona di Pregio appare pressoché impossibile, con le soglie di sostenibilità al di sotto o estremamente vicine ai minimi di canone concordato (e a maggior ragione di mercato). Occorre dunque allontanarsi dal centro, giovandosi anche dell'aumento del reddito familiare netto negli ultimi anni del periodo, combinato con il calo dei prezzi iniziato tra 2013 e 2014. La Zona A offre dunque monolocali accessibili (seppur con margini risicati rispetto ai valori minimi di canone), mentre la Zona B garantisce buone possibilità di accedere a monolocali e bilocali (dal 2014). 
Figura 37 - Bologna, Zona di pregio. Serie storica dei prezzi di affitto potenziali minimi e massimi, a canone concordato e di mercato, suddivisi per numero di vani. Soglie di sostenibilità (30\% reddito familiare mensile netto) per nuclei di riferimento.

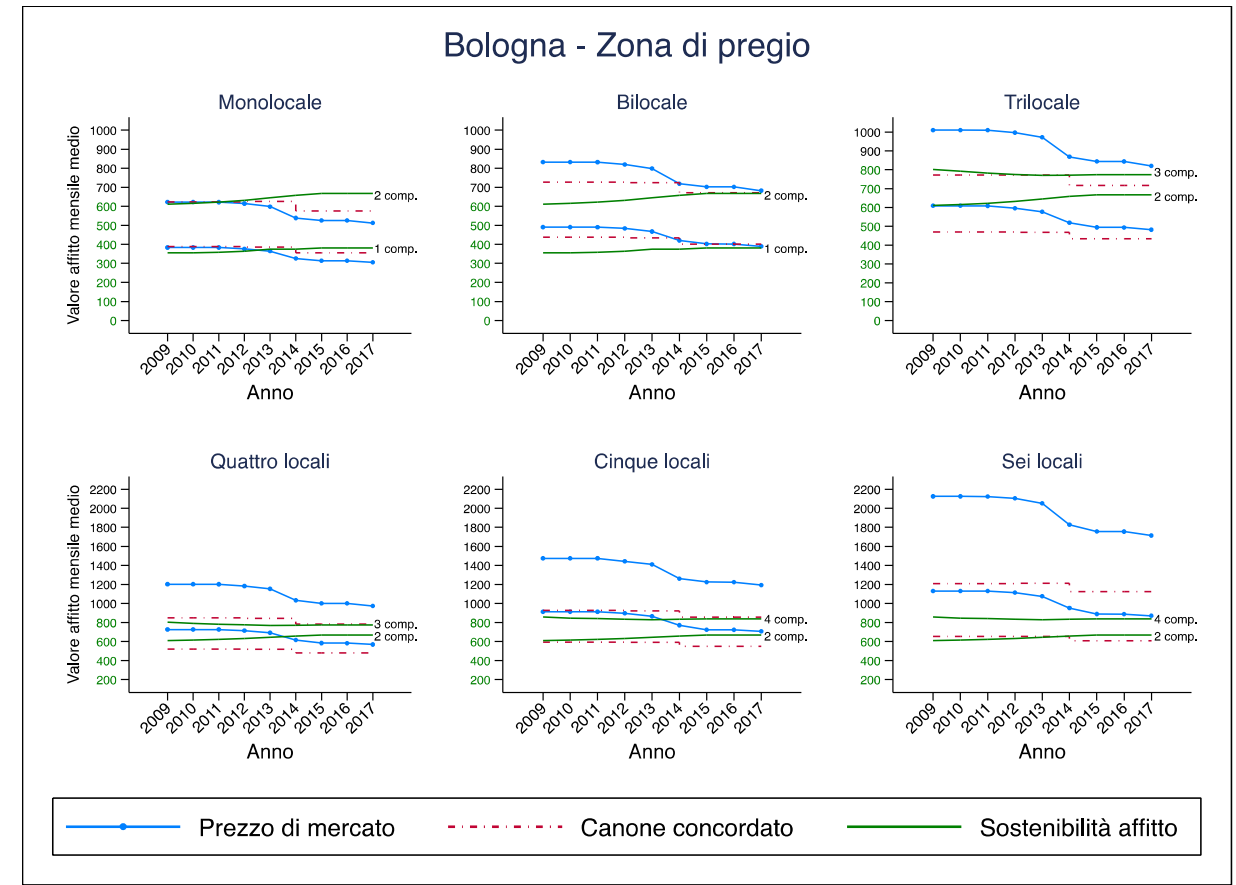

Figura 38 -Bologna, Zona A. Serie storica dei prezzi di affitto potenziali minimi e massimi, a canone concordato e di mercato, suddivisi per numero di vani. Soglie di sostenibilità (30\% reddito familiare mensile netto) per nuclei di riferimento.

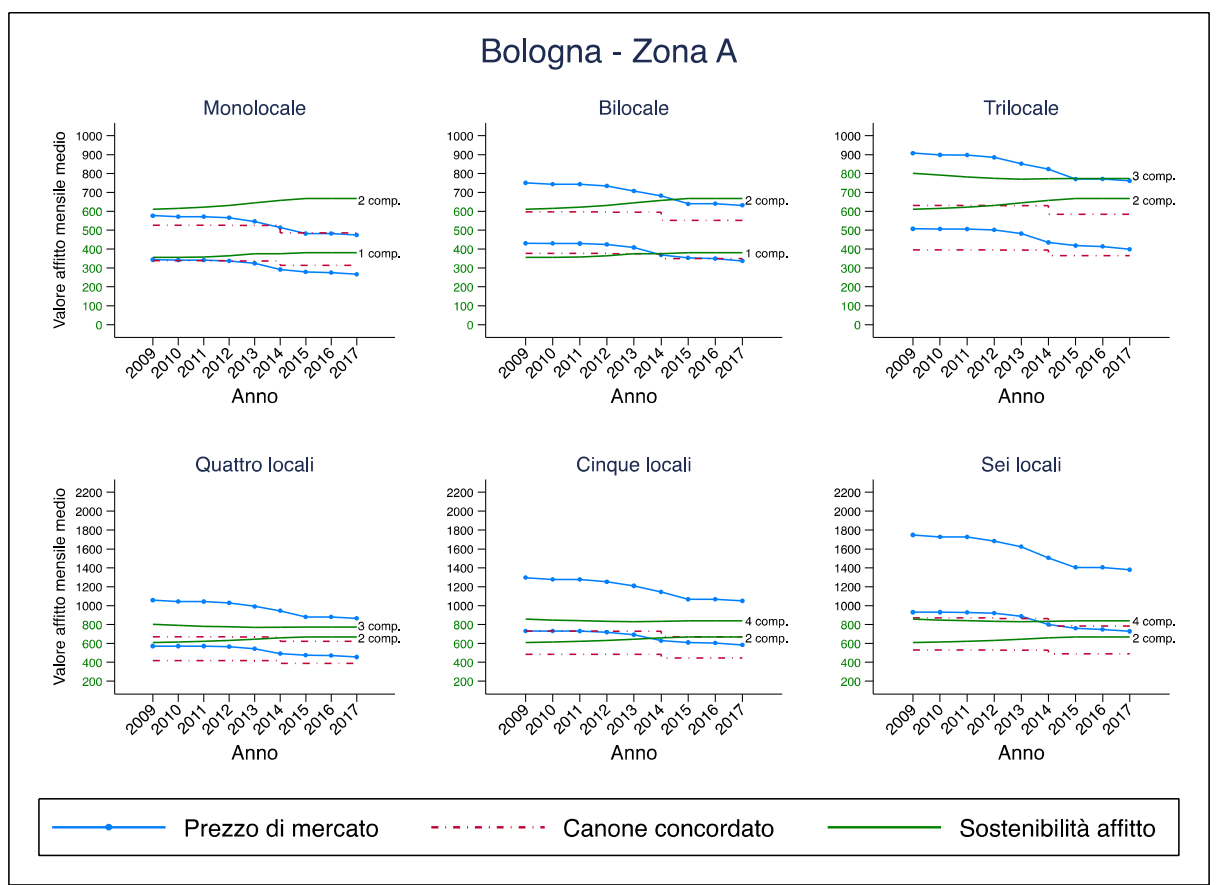


Figura 39 - Bologna, Zona B. Serie storica dei prezzi di affitto potenziali minimi e massimi, a canone concordato e di mercato, suddivisi per numero di vani. Soglie di sostenibilità (30\% reddito familiare mensile netto) per nuclei di riferimento.

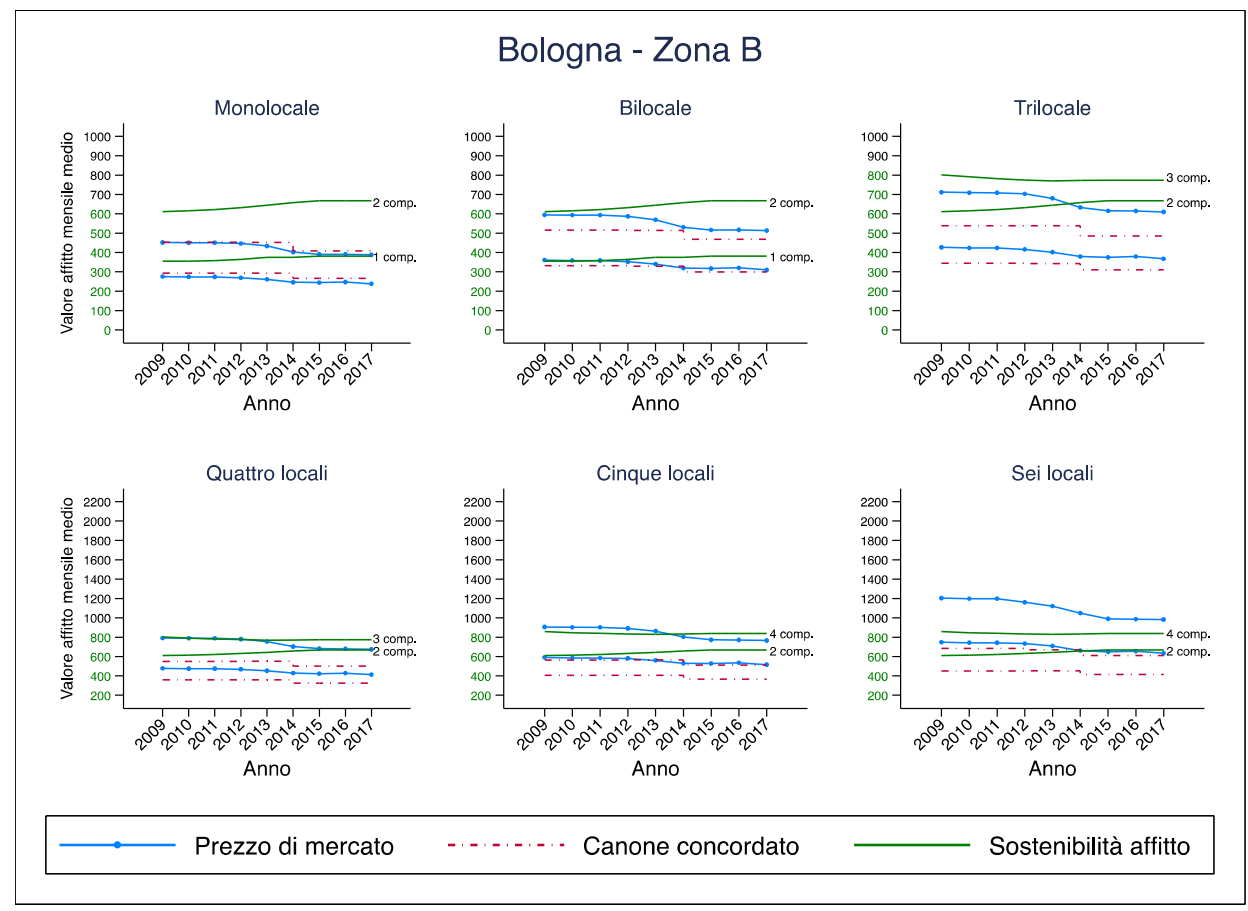




\section{Le regole di equilibri di mercato per il canone concordato}

Dopo aver presentato l'evoluzione dei prezzi al metro quadro e dei canoni d'affitto mensili dei contratti di locazione a canone libero e canone di mercato, questa parte della ricerca si prefigge l'obiettivo di valutare sostenibilità e concorrenzialità del canone concordato. Date per presenti sia una certa domanda di locazioni abitative sia una certa offerta, affinché un contratto d'affitto - a libero mercato o a canone concordato - venga stipulato, è necessario che si verifichino due precise condizioni, una dal lato della domanda, l'altra dal lato dell'offerta. Tali condizioni sono quelle che qui vengono definite la concorrenzialità dal lato dell'offerta e la sostenibilità dal lato della domanda. Concorrenzialità e sostenibilità infatti non sono, se considerate singolarmente, condizioni sufficienti per definire il numero effettivo di abitazioni affittabili mediante canone concordato. Obiettivo di questo paragrafo è quello di esaminare congiuntamente queste due condizioni, per offrire una valutazione più completa del mercato in esame.

La sostenibilità di un canone di locazione interessa in particolare il locatario, riguarda quindi il lato della domanda del mercato locativo. La sostenibilità, come anticipato nella sezione precedente, viene definita in letteratura. ${ }^{49} \mathrm{E}$ "sostenibile" un'abitazione il cui affitto mensile medio (a canone concordato o di mercato) sia inferiore al $30 \%$ del reddito familiare mensile netto.

L'analisi della sostenibilità sarà affrontata in un primo momento avendo come riferimento la famiglia bolognese mediana, con l'obiettivo di valutare la situazione complessiva del mercato cittadino. Oltre a questo nucleo familiare di riferimento, è interesse di questa ricerca approfondire le possibilità di accesso agli alloggi per famiglie in difficoltà economica e sociale, che non trovano risposta immediata nell'edilizia residenziale pubblica in quanto non sufficientemente ben posizionate nelle graduatorie ERP. Queste famiglie versano in condizioni economiche che non garantiscono loro una piena autonomia sul mercato delle locazioni (a canone concordato e, a maggior ragione, a canone libero) e saranno analizzate nel prossimo paragrafo.

\footnotetext{
${ }^{49}$ La definizione è data da Banca d'Italia nelle pubblicazioni biennali di commento all'Indagine sui Bilanci delle famiglie italiane, chiamate "Supplemento al bollettino statistico". Si veda ad esempio "I bilanci delle famiglie italiane nell'anno 2012", n.5, 27 gennaio 2014 (p.27):

https://www.bancaditalia.it/pubblicazioni/indagine-famiglie/bil-fam2012/suppl 05 14n.pdf

In questa pubblicazione Banca d'Italia stessa rimanda alla consultazione di M. Schwartz ed E. Wilson, "Who can afford a home?", US Census Bureau:

https://www.census.gov/hhes/www/housing/special-topics/files/who-can-afford.pdf
} 
Il secondo tema d'interesse affrontato in questa parte della ricerca riguarda la condizione di profitto da parte del locatore, ossia dal lato dell'offerta. La sostenibilità, da sola, non è sufficiente per garantire l'accesso delle famiglie alle abitazioni: affinché avvenga una stipula del contratto è necessario che vi sia anche una soddisfazione delle condizioni poste dal conduttore. Quest'ultimo, infatti, potrebbe avere proposte migliori al di fuori del regime di canone concordato e decidere di non ricorrere a questa forma contrattuale. ${ }^{50}$ É definita "concorrenziale" un'abitazione il cui canone concordato medio al netto della cedolare secca (pari al 19\% fino al 2013, poi scesa al $10 \%$ dal 2014) è maggiore o uguale al canone medio a libero mercato, sempre al netto della cedolare secca al $21 \%$ prevista per questa tipologia di contratti.

Va sottolineato tuttavia che anche all'interno dei canoni concordati esiste un incentivo (quello che gli economisti definiscono una strategia dominante) per i conduttori (in base alla loro avversione al rischio) ad affittare a studenti universitari a canone concordato piuttosto che a famiglie. Questo incentivo è sia di natura sociodemografica che legislativa. Dal punto di vista socio-demografico, le famiglie rappresentano un soggetto che genera maggiori rischi per il conduttore. La presenza di minorenni, la forte dipendenza dei nuclei familiari da redditi omogenei da lavoro, la maggiore esposizione a disabilità e disagio, legalmente tutelati, rendono le famiglie un soggetto di cui è più complesso "liberarsi" laddove vengano meno gli accordi sottoscritti. Dal punto di vista di soggetti avversi al rischio, come sono generalmente i locatori di immobili (quantomeno i piccoli proprietari - problematica che emerge sia nella parte di indagine di mercato che nella letteratura economica) la famiglia è un contraente più pericoloso di uno studente.

Si aggiunge a questa osservazione l'aspetto legale. Lo studente al fine di ottenere il contratto a canone concordato non deve avere la residenza nell'area del comune. Appare agli occhi del locatore molto più semplice ritenere lo studente un soggetto più debole come contraente e quindi più facilmente "gestibile" rispetto ad una famiglia. Tuttavia questo aspetto si scontra con la variazione dei parametri su cui è calcolato il pagamento delle tasse universitarie. II passaggio all'ISEE infatti pare aver contribuito fortemente ad un incremento di soggetti a ISEE 0 fra gli iscritti all'Alma Mater Studiorum. In prima valutazione appare possibile che questo accada a causa della creazione di nuovi nuclei familiari indipendenti dalle famiglie di origine.

Diversamente dalla discussione precedente, per valutare concorrenzialità e sostenibilità del canone concordato, volendo quantificare per ogni zona il numero effettivo di abitazioni sostenibili e concorrenziali, si adotta la definizione mutuamente esclusiva del numero di vani già discussa in precedenza.

\footnotetext{
50 Implicitamente si sta assumendo che vi sia sempre profitto nel porre sul mercato di locazione una abitazione sfitta; questa condizione tuttavia non può essere verificata sulla base dati infomativa in nostro possesso. Facendo riferimento solo all'indagiine conoscitiva nella prima parte sappiamo esservi una quota, intorno al $10 \%$ delle abitazioni, non sul mercato locativo né adibite ad altro utilizzo, tuttavia non si dispone di ulteriori informazioni su tale patrimonio immobiliare.
} 


\section{Soglie di sostenibilità}

Al fine di valutare la condizione del cittadino mediano, questa ricerca si rifà agli studi annualmente pubblicati dall'Area Programmazione Controlli e Statistica del Comune di Bologna ${ }^{51}$, relativi ai redditi dichiarati dalle famiglie bolognesi. Da questi si è ricavato il reddito mediano equivalente pro-capite, anno per anno (per il 2016 e 2017 è applicato il valore 2015, ultimo disponibile ad oggi). II dato viene poi lavorato scontandone l'aliquota IRPEF e moltiplicandolo per i coefficienti della scala OCSE modificata per tenere in conto del numero di adulti. Infine, una volta desunto il reddito mensile, si individua, come da letteratura, il 30\% dello stesso quale tetto massimo per le spese d'affitto ${ }^{52}$.

Sono state calcolate le soglie di sostenibilità economica per 4 differenti tipologie familiari a seconda del numero di membri componenti il nucleo (da 1 a 4). Ad ogni tipologia di immobile (numero di vani) vengono associate le soglie massime per due nuclei di riferimento: quelli di due persone (ossia l'ampiezza media delle famiglie bolognesi), e quelli corrispondenti alla grandezza delle abitazioni (1 persona per i monolocali e i bilocali; 3 persone per i trilocali e quadrilocali; 4 dai cinque locali in su).

Figura 40 - Evoluzione temporale soglie di sostenibilità, cittadino bolognese mediano.

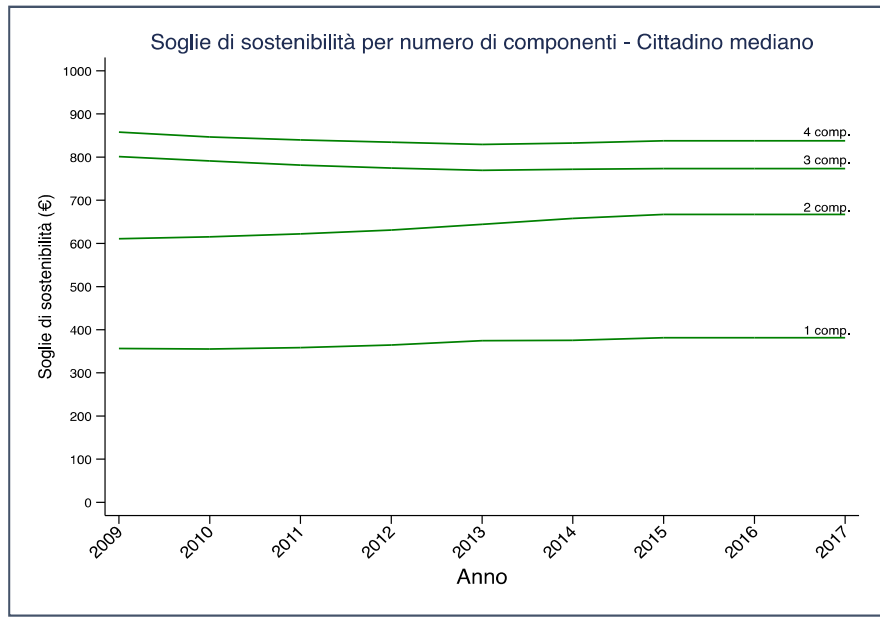

La sostenibilità viene poi calcolata in funzione della soglia maggiore tra le due: 2 persone per monolocali e bilocali, 3 persone per tre e quattro locali, 4 persone per le

\footnotetext{
51 "I redditi 2015 dichiarati dalle cittadine e dai cittadini di Bologna", Comune di Bologna, Area Programmazione Controlli e Statistica, Dicembre 2017,: http://www.comune.bologna.it/iperbole/piancont/Redditi/PRES REDDITI 2015var50.pdf 52 Per una trattazione più dettagliata relativa alla determinazione di queste soglie si rimanda alla nota metodologica.
} 
abitazioni più grandi. Ovviamente si tratta di assunzioni necessarie ad inquadrare "in media" il problema. Ogni singola famiglia infatti presenta specifiche esigenze. La Figura 40 illustra l'evoluzione temporale delle 4 soglie determinate in base al numero di componenti53.

La Tabella 36 rappresenta il numero di abitazioni totali, I'affitto medio e la percentuale di abitazioni sostenibili a canone concordato, degli stock di alloggi 2010, 2012 e 2014, suddivise per zone di applicazione del canone e per numero di vani. Tali soglie sono tuttavia definite sulla base di conversione dei redditi mediani rispetto alle famiglie bolognesi dove spesso famiglie di 3 persone comprendono 3 lavoratori (2 genitori e un figlio), tuttavia nella realtà dei fatti le famiglie di 3 o 4 persone potrebbero avere de facto valori molto più bassi laddove le fonti di reddito siano 2 o meno.

Tabella 36 - Percentuale di abitazioni sostenibili a canone concordato, suddivise per zone di applicazione del canone concordato e numero di vani.

\begin{tabular}{|c|c|c|c|c|c|c|c|c|c|c|}
\hline \multirow[b]{3}{*}{$\begin{array}{l}\text { Zona } \\
\text { Canone }\end{array}$} & & \multicolumn{9}{|c|}{ Abitazioni sostenibili a canone concordato } \\
\hline & & \multicolumn{3}{|c|}{2010} & \multicolumn{3}{|c|}{2012} & \multicolumn{3}{|c|}{2014} \\
\hline & Vani & Tot. & $\begin{array}{l}\text { Affitto } \\
\text { medio }\end{array}$ & $\begin{array}{c}\% \\
\text { Sost. }\end{array}$ & Tot. & $\begin{array}{l}\text { Affitto } \\
\text { medio }\end{array}$ & $\begin{array}{c}\% \\
\text { Sost. }\end{array}$ & Tot. & $\begin{array}{l}\text { Affitto } \\
\text { medio }\end{array}$ & $\begin{array}{c}\% \\
\text { Sost. }\end{array}$ \\
\hline \multirow{6}{*}{ 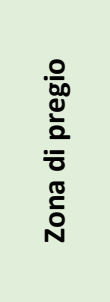 } & 1 & 468 & 450.8 & 100.0 & 536 & 450.2 & 100.0 & 628 & 415.7 & 100.0 \\
\hline & 2 & 287 & 526.3 & 100.0 & 318 & 527.9 & 100.0 & 368 & 488.2 & 100.0 \\
\hline & 3 & 624 & 590.3 & 100.0 & 640 & 588.1 & 100.0 & 767 & 544.7 & 100.0 \\
\hline & 4 & 560 & 689.0 & 100.0 & 576 & 688.9 & 100.0 & 686 & 637.6 & 100.0 \\
\hline & 5 & 437 & 755.2 & 100.0 & 423 & 754.7 & 100.0 & 517 & 702.2 & 100.0 \\
\hline & 6 & 635 & 984.4 & 38.7 & 626 & 985.2 & 36.1 & 767 & 913.3 & 50.7 \\
\hline \multirow{6}{*}{ 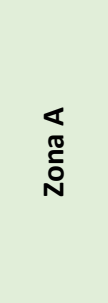 } & 1 & 966 & 383.4 & 100.0 & 989 & 382.1 & 100.0 & 1165 & 353.3 & 100.0 \\
\hline & 2 & 864 & 434.1 & 100.0 & 860 & 433.5 & 100.0 & 1074 & 401.4 & 100.0 \\
\hline & 3 & 2491 & 480.5 & 100.0 & 2452 & 480.4 & 100.0 & 2999 & 445.5 & 100.0 \\
\hline & 4 & 1916 & 566.5 & 100.0 & 1838 & 566.5 & 100.0 & 2265 & 525.8 & 100.0 \\
\hline & 5 & 963 & 625.9 & 100.0 & 929 & 625.1 & 100.0 & 1154 & 576.2 & 100.0 \\
\hline & 6 & 820 & 770.0 & 78.5 & 766 & 767.1 & 77.3 & 907 & 703.7 & 86.9 \\
\hline \multirow{6}{*}{ 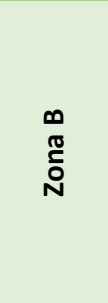 } & 1 & 1203 & 329.2 & 100.0 & 1211 & 328.5 & 100.0 & 1469 & 297.8 & 100.0 \\
\hline & 2 & 1179 & 375.2 & 100.0 & 1188 & 374.5 & 100.0 & 1461 & 340.3 & 100.0 \\
\hline & 3 & 3716 & 418.4 & 100.0 & 3497 & 417.6 & 100.0 & 4406 & 377.3 & 100.0 \\
\hline & 4 & 2632 & 483.0 & 100.0 & 2534 & 482.7 & 100.0 & 3207 & 438.9 & 100.0 \\
\hline & 5 & 799 & 531.3 & 100.0 & 764 & 531.9 & 100.0 & 983 & 482.5 & 100.0 \\
\hline & 6 & 310 & 669.0 & 91.9 & 304 & 653.5 & 92.1 & 361 & 602.0 & 93.6 \\
\hline
\end{tabular}

Dalla Tabella 36, indipendente dalla zona di applicazione del canone concordato considerata, tutte le abitazioni fino a 5 locali appaiono economicamente sostenibili per il nucleo familiare di riferimento. Solamente le abitazioni a sei locali, per

\footnotetext{
${ }^{53}$ Nella nota metodologica viene presentata la tabella con i valori rappresentati in questo grafico.
} 
qualunque anno considerato, presentano una percentuale di abitazioni sostenibili inferiore al $100 \%$. Queste percentuali differiscono notevolmente per le diverse zone e solo leggermente tra i diversi anni (in particolare tra 2010 e 2012). Relativamente al 2012, la famiglia mediana di quattro componenti ${ }^{54}$ può permettersi solo il $36.10 \%$ dei sei locali nella zona di pregio, il $77.29 \%$ in zona A e il $92.11 \%$ in zona B. Le percentuali relative allo stock di alloggi 2010 sono simili; quelle relative al 2014 sono maggiori per ognuna delle tre zone, indicando un miglioramento della sostenibilità economica degli affitti in questo anno.

La maggior sostenibilità registrata nel 2014 deriva dalla riduzione dei canoni di locazione mensili, a sua volta quasi sicuramente determinata dalla revisione al ribasso dei valori massimi e minimi definiti dalla revisione dell'accordo per l'applicazione dei contratti a canone concordato.

Le differenze all'interno dei singoli anni erano prevedibili in quanto l'accordo di applicazione dei contratti di canone concordato prevede prezzi inferiori spostandosi dalla prima all'ultima zona.

Secondo tali valori, la coppia bolognese mediana può sostenere tutti gli affitti a canone concordato per le abitazioni di piccole dimensioni (monolocali e bilocali); il nucleo mediano composto da tre persone può sostenere tutti gli affitti per le medie dimensioni (tre e quattro vani), oltre alle abitazioni di dimensioni inferiori, per quanto non adatte a questa tipologia familiare. La famiglia mediana di 4 persone, invece, può sostenere l'affitto a canone concordato in tutti i 5 locali, e nelle abitazioni più piccole per quanto alcune non adatte a loro, ma non nella totalità dei 6 locali.

La definizione di sostenibilità utilizzata merita una precisazione su tre questioni che possono determinare questi alti tassi di sostenibilità. La prima è la definizione del valore del canone utilizzata: si tratta del canone medio, calcolato a partire dai valori minimi e massimi precedentemente utilizzati. Anche se tutte le abitazioni risultano sostenibili rispetto al canone concordato medio, non è detto che in tutti i contratti sia applicato questo valore, e le percentuali sarebbero sicuramente diverse considerando l'estremo superiore in sostituzione alla media.

La seconda questione riguarda i redditi utilizzati per la definizione delle soglie: si tratta dei redditi mediani dei cittadini bolognesi. Pur essendo il miglior indicatore possibile date le informazioni disponibili, non riesce comunque a cogliere le differenze tra tipologie familiari differenti. Una famiglia di 4 persone potrebbe essere composta da 4 percettori di reddito (si pensi ad esempio a due genitori, un figlio che lavora e un pensionato), ma allo stesso tempo potrebbe essere una coppia con due figli piccoli.

\footnotetext{
${ }^{54}$ Per come è stata definita la sostenibilità la soglia per i 5 e 6 locali è definita in base al nucleo familiare mediano di 4 persone.
} 
evidente che le percentuali calcolate sovrastimano la sostenibilità per famiglie di questo tipo ${ }^{55}$.

L'ultima questione riguarda la soglia utilizzata a seconda del numero di vani: questa è stata definita in funzione del numero di persone più adatto alla tipologia di abitazione, considerando sempre il valore massimo quando presenti più possibilità ${ }^{56}$. Considerando però la composizione delle famiglie nel comune di Bologna, i nuclei di 3 e 4 persone rappresentano rispettivamente il $13.1 \%$ e $7.7 \%$ sul totale delle famiglie. È quindi interessante definire la sostenibilità in funzione della coppia mediana (poiché 2 è il numero medio di membri per famiglia), indipendentemente dal numero di vani.

Tabella 37 - Percentuale di abitazioni sostenibili a canone concordato per il nucleo mediano di due persone, suddivise per zone di applicazione del canone concordato e numero di vani.

\begin{tabular}{|c|c|c|c|c|c|c|c|c|c|c|c|c|c|}
\hline \multicolumn{14}{|c|}{ Abitazioni sostenibili a canone concordato } \\
\hline \multirow[b]{2}{*}{ 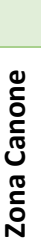 } & \multirow[b]{2}{*}{$\begin{array}{l}\bar{c} \\
\text { 永 }\end{array}$} & \multicolumn{4}{|c|}{2010} & \multicolumn{4}{|c|}{2012} & \multicolumn{4}{|c|}{2014} \\
\hline & & 붐 & 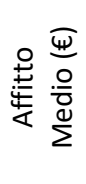 & $\begin{array}{l}\stackrel{+}{n} \\
\text { ஸे } \\
z\end{array}$ & $\begin{array}{l}\text { ஸें } \\
\text { ஸे } \\
\text { dீ }\end{array}$ & $\stackrel{+}{\circ}$ & $\underbrace{\frac{\overparen{\Psi}}{4}}_{\frac{ \pm}{4}}$ & $\begin{array}{l}+் \\
\text { D̃ } \\
z\end{array}$ & 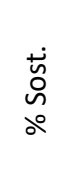 & $\stackrel{+}{\circ}$ & 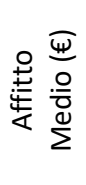 & $\begin{array}{l}+\pi \\
\text { D̃ } \\
z\end{array}$ & 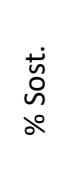 \\
\hline \multirow{6}{*}{ 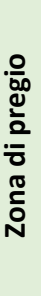 } & 1 & 468 & 451 & 468 & 100 & 536 & 450 & 536 & 100 & 628 & 416 & 628 & 100 \\
\hline & 2 & 287 & 526 & 287 & 100 & 318 & 528 & 318 & 100 & 368 & 488 & 368 & 100 \\
\hline & 3 & 624 & 590 & 396 & 64 & 640 & 588 & 488 & 76 & 767 & 545 & 767 & 100 \\
\hline & 4 & 560 & 689 & 0 & 0 & 576 & 689 & 67 & 12 & 686 & 638 & 435 & 63 \\
\hline & 5 & 437 & 755 & 0 & 0 & 423 & 755 & 0 & 0 & 517 & 702 & 52 & 10 \\
\hline & 6 & 635 & 984 & 0 & 0 & 626 & 985 & 0 & 0 & 767 & 913 & 0 & 0 \\
\hline \multirow{6}{*}{$\begin{array}{l}\frac{1}{\pi} \\
\stackrel{0}{5} \\
\text { N }\end{array}$} & 1 & 966 & 383 & 966 & 100 & 989 & 382 & 989 & 100 & 1165 & 353 & 1165 & 100 \\
\hline & 2 & 864 & 434 & 864 & 100 & 860 & 434 & 860 & 100 & 1074 & 401 & 1074 & 100 \\
\hline & 3 & 2491 & 481 & 2491 & 100 & 2452 & 480 & 2452 & 100 & 2999 & 445 & 2999 & 100 \\
\hline & 4 & 1916 & 567 & 1691 & 88 & 1838 & 567 & 1766 & 96 & 2265 & 529 & 2265 & 100 \\
\hline & 5 & 963 & 626 & 463 & 48 & 929 & 625 & 564 & 61 & 1154 & 576 & 1154 & 100 \\
\hline & 6 & 820 & 770 & 10 & 1 & 766 & 767 & 76 & 10 & 907 & 704 & 503 & 56 \\
\hline \multirow{6}{*}{$\begin{array}{c}\infty \\
\mathbb{0} \\
\stackrel{c}{0} \\
N\end{array}$} & 1 & 1203 & 329 & 1203 & 100 & 1211 & 326 & 1211 & 100 & 1469 & 298 & 1469 & 100 \\
\hline & 2 & 1179 & 375 & 1179 & 100 & 1188 & 376 & 1188 & 100 & 1461 & 340 & 1461 & 100 \\
\hline & 3 & 3716 & 418 & 3716 & 100 & 3497 & 418 & 3497 & 100 & 4406 & 377 & 4406 & 100 \\
\hline & 4 & 2632 & 483 & 2632 & 100 & 2534 & 483 & 2534 & 100 & 3207 & 439 & 3207 & 100 \\
\hline & 5 & 799 & 531 & 799 & 100 & 764 & 532 & 764 & 100 & 983 & 483 & 983 & 100 \\
\hline & 6 & 310 & 669 & 179 & 58 & 304 & 654 & 201 & 66 & 361 & 602 & 300 & 83 \\
\hline
\end{tabular}

\footnotetext{
${ }^{55}$ Lo stesso discorso può valere per i nuclei di dimensione inferiore: anche una coppia, ad esempio, potrebbe essere monoreddito e difficilmente raggiungere queste elevate percentuali di sostenibilità.

${ }^{56}$ Per i monolocali e bilocali si adotta la soglia relativa ad una coppia, per i tre e quattro locali si applica il valore del nucleo mediano composto da 3 persone e la famiglia di 4 persone è adottata per le restanti tipologie.
} 
La Tabella 37 riporta gli stessi valori di quella precedente, con riferimento ad una soglia di sostenibilità definita sul nucleo mediano composto da 2 persone.

Il numero di abitazioni sostenibili si riduce, indipendentemente dal numero di vani, considerando come riferimento il nucleo mediano di due persone. Si riducono, per la Zona di Pregio e la Zona A, il numero di tipologie abitative per cui tutte le abitazioni rientrano al di sotto della soglia del $30 \%$ del reddito medio. Tralasciando le abitazioni a 5 o 6 vani, difficilmente richieste effettivamente da un nucleo di due persone, e quelle a 1 o 2 vani per cui tutte le abitazioni continuano ad essere sostenibili, è interessante soffermarsi sui trilocali e le abitazioni di 4 vani: si tratta, infatti, di tipologie di abitazioni che potrebbero essere effettivamente domandate dalle coppie. Entrambe queste tipologie di alloggi risultano interamente sostenibili per la Zona B, e i trilocali anche nella Zona A. Relativamente ai quattro locali della zona A la percentuale di abitazioni sostenibili rimane comunque elevata e aumenta con gli anni, raggiungendo nel 2014 la completa sostenibilità anche per i trilocali. La Zona di Pregio, per cui i prezzi al metro quadro del canone concordato sono più alti, presenta percentuali nettamente inferiori, ma anche in questo caso la quota di abitazioni sostenibili aumenta tra il 2010 e il 2014.

In questa tabella, inoltre, anche le differenze delle percentuali di abitazioni sostenibili tra i diversi stock risultano maggiori rispetto alla Tabella 36. In generale il mercato a canone concordato nel 2012 appare più sostenibile (rispetto al 2010) per la famiglia mediana: escludendo le percentuali analoghe ( $0 \%$ e 100\%), per tutte le percentuali differenti lo stock di alloggi 2012 presenta una percentuale di abitazioni sostenibili maggiore, qualunque sia la zona o il numero di vani. A sua volta lo stock di alloggi 2014 risulta maggiormente sostenibile se confrontato al 2012.

Può essere utile identificare il numero di abitazioni effettivamente sostenibili per la coppia mediana, considerando il 2014 come riferimento in quanto i prezzi al metro quadro del canone per questo anno sono analoghi a quelli attuali ${ }^{57}$. Nella Zona di Pregio 2'250 delle 3'733 abitazioni locate a canone concordato, ossia il $60.27 \%$ del totale, risultano sostenibili. II 100\% delle abitazioni di piccole dimensioni (1 o 2 vani) sono sostenibili (996 abitazioni), 1'202 sono accessibili tra le abitazioni di medie dimensioni e solo 52 tra quelle grandi.

Il numero di abitazioni sostenibili, sia in termini assoluti che percentuali, aumenta passando alla Zona A: 9'160 di 9'564 (95.78\%). Tutte le abitazioni piccole e medie risultano sostenibili per la coppia mediana, mentre di quelle grandi le sostenibili sono 1'657 su 2'061. Relativamente alla zona B il 99.49\%, ossia 11'826, risultano sostenibili: solamente 61 a sei locali non soddisfano la soglia di spesa mensile della coppia mediana. Complessivamente delle $25^{\prime} 184$ abitazioni locate a canone concordato nel

\footnotetext{
57 A tal proposito si sottolinea ancora una volta come la riduzione dei prezzi al metro quadro determini le maggiori percentuali di sostenibilità in corrispondenza di questo anno rispetto a quelli precedenti.
} 
Comune di Bologna, 23'236 (92.26\%) risultano accessibili alla coppia mediana bolognese.

È importante sottolineare ancora una volta come questa sostenibilità sia determinata dall'impianto metodologico su cui si basa la ricerca. In primo luogo, la coppia mediana per quanto utile per l'analisi non rappresenta tutte le famiglie della città: esistono, tra i tanti nuclei di due persone, anche coppie monoreddito che difficilmente potranno registrare percentuali di sostenibilità così elevate. Inoltre, per definire la sostenibilità è stato utilizzato il canone mensile medio, ottenuto come sintesi del valore più basso in assoluto (prezzo al metro quadro minimo della fascia più bassa di elementi di pregio) e quello più alto (prezzo al metro quadro massimo della fascia con il maggior numero di elementi di pregio). Non avendo informazioni in merito ai contratti stipulati non è stato possibile procedere diversamente, ma in assenza di informazioni sugli elementi di pregio la differenza nella sostenibilità può essere consistente (è altresì possibile il contrario).

Poiché i nuclei familiari di 3 o 4 persone hanno soglie di sostenibilità maggiori rispetto a quella della coppia mediana, il numero di abitazioni sostenibili per queste due famiglie di riferimento è ancora più elevato. Tuttavia, come detto, le famiglie di 3 o 4 membri hanno redditi mediani calcolati tramite $\mathrm{i}$ coefficienti di conversione che potrebbero fortemente sovrastimare la condizione economica familiare. Infine, il nucleo individuale presenta una possibilità di spesa nettamente inferiore alle altre famiglie: 381.26€ mensili secondo l'ultimo dato disponibile (2015). Le percentuali di sostenibilità relative al nucleo individuale, che rappresenta il $51.4 \%$ percento delle famiglie bolognesi, sono riportate nella Tabella 38.

Come prevedibile, il numero di abitazioni sostenibili per la famiglia mediana unipersonale sono nettamente più basse rispetto a quelle precedentemente calcolate. Per nessun anno analizzato e per nessuna delle tre zone di applicazione del canone concordato alcuna abitazione da 4 vani o più risulta accessibile. Le percentuali di sostenibilità aumentano tra il 2010 e il 2014 e passando dalla zona di pregio alla Zona B. Relativamente alla zona di pregio, solamente il $6.41 \%$ dei monolocali risulta accessibile nel 2010 a questa famiglia di riferimento.

La situazione migliora leggermente passando al 2014, quando la percentuale di monolocali sostenibili sale al $28.66 \%$. Nessuna delle abitazioni più grandi risulta sostenibile per questo nucleo familiare. Nella Zona A si passa dal $31.06 \%$ di monolocali sostenibili nel 2010 al 60\% degli stessi nel 2014, anno in cui risultano accessibili anche il $13.50 \%$ dei bilocali. La Zona B presenta le percentuali più alte: nel 2014 la totalità di monolocali e bilocali di quest'area risulta accessibile, unitamente al $47.32 \%$ dei trilocali. Per rendere chiara l'idea: Bologna non è una città per single o per famiglie monoreddito. Questo risultato appare particolarmente importante; infatti segnala una strutturale impossibilità per le nuove generazioni di abbandonare il tetto familiare laddove non vi sia un partner o altre persone con cui condividere i costi (non solo nel centro cittadino ma nella gran parte dell'area urbana), e costringe gli anziani soli, 
laddove non dotati di pensioni particolarmente elevate o proprietari della propria abitazione, a oggettive difficoltà.

Tabella 38. Percentuale di abitazioni sostenibili a canone concordato per il nucleo mediano individuale, suddivise per zone di applicazione del canone concordato e numero di vani.

\begin{tabular}{|c|c|c|c|c|c|c|c|c|c|c|c|c|c|}
\hline \multicolumn{14}{|c|}{ Abitazioni sostenibili a canone concordato per nucleo mediano di 1 persona } \\
\hline \multirow[b]{2}{*}{ 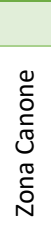 } & \multirow[b]{2}{*}{$\begin{array}{l}\overline{\bar{c}} \\
\stackrel{5}{>}\end{array}$} & \multicolumn{4}{|c|}{2010} & \multicolumn{4}{|c|}{2012} & \multicolumn{4}{|c|}{2014} \\
\hline & & ث் & 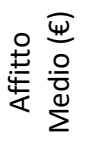 & $\begin{array}{l}\dot{\tilde{\omega}} \\
\stackrel{0}{n} \\
z\end{array}$ & 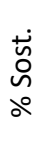 & ثீं & 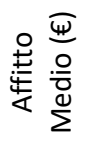 & $\begin{array}{l}\tilde{\Delta} \\
\text { ஸे } \\
z\end{array}$ & $\begin{array}{l}\text { में } \\
\text { ஸे } \\
\text { do }\end{array}$ & ப் & 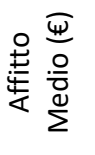 & $\begin{array}{l}\dot{\tilde{n}} \\
\text { ஸे } \\
z\end{array}$ & $\begin{array}{l}\dot{\omega} \\
\text { ஸे } \\
\text { よீ }\end{array}$ \\
\hline \multirow{6}{*}{$\begin{array}{l}\frac{0}{00} \\
\frac{0}{20} \\
\frac{0}{2} \\
\frac{0}{0} \\
\frac{0}{0} \\
\text { N }\end{array}$} & 1 & 468 & 451 & 30 & 6 & 536 & 450 & 58 & 11 & 628 & 416 & 180 & 29 \\
\hline & 2 & 287 & 526 & 0 & 0 & 318 & 528 & 0 & 0 & 368 & 488 & 0 & 0 \\
\hline & 3 & 624 & 590 & 0 & 0 & 640 & 588 & 0 & 0 & 767 & 545 & 0 & 0 \\
\hline & 4 & 560 & 689 & 0 & 0 & 576 & 689 & 0 & 0 & 686 & 638 & 0 & 0 \\
\hline & 5 & 437 & 755 & 0 & 0 & 423 & 755 & 0 & 0 & 517 & 702 & 0 & 0 \\
\hline & 6 & 635 & 984 & 0 & 0 & 626 & 985 & 0 & 0 & 767 & 913 & 0 & 0 \\
\hline \multirow{6}{*}{ 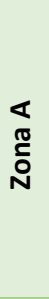 } & 1 & 966 & 383 & 300 & 31 & 989 & 382 & 344 & 35 & 1165 & 353 & 699 & 60 \\
\hline & 2 & 864 & 434 & 0 & 0 & 860 & 433 & 0 & 0 & 1074 & 401 & 145 & 14 \\
\hline & 3 & 2491 & 480 & 0 & 0 & 2452 & 480 & 0 & 0 & 2999 & 446 & 0 & 0 \\
\hline & 4 & 1916 & 566 & 0 & 0 & 1838 & 567 & 0 & 0 & 2265 & 526 & 0 & 0 \\
\hline & 5 & 963 & 626 & 0 & 0 & 929 & 625 & 0 & 0 & 1154 & 576 & 0 & 0 \\
\hline & 6 & 820 & 770 & 0 & 0 & 766 & 767 & 0 & 0 & 907 & 704 & 0 & 0 \\
\hline \multirow{6}{*}{ 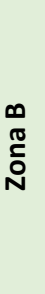 } & 1 & 1203 & 329 & 781 & 65 & 1211 & 328 & 908 & 75 & 1469 & 298 & 1469 & 100 \\
\hline & 2 & 1179 & 375 & 253 & 21 & 1188 & 375 & 407 & 34 & 1461 & 340 & 1461 & 100 \\
\hline & 3 & 3716 & 418 & 0 & 0 & 3497 & 418 & 160 & 5 & 4406 & 377 & 2085 & 47 \\
\hline & 4 & 2632 & 483 & 0 & 0 & 2534 & 483 & 0 & 0 & 3207 & 439 & 0 & 0 \\
\hline & 5 & 799 & 531 & 0 & 0 & 764 & 532 & 0 & 0 & 983 & 482 & 0 & 0 \\
\hline & 6 & 310 & 669 & 0 & 0 & 304 & 654 & 0 & 0 & 361 & 602 & 0 & 0 \\
\hline
\end{tabular}

Complessivamente, facendo riferimento al 2014, le abitazioni accessibili alla famiglia unitaria mediana sono 180 in Zona di Pregio (ossia il 4.8\% dello stock di abitazioni locate a canone concordato in quest'area), 844 in Zona A (8.83\% delle abitazioni di questa zona) e 5'015 (42.19\%) in Zona B. Complessivamente nel Comune di Bologna solo 6'039 delle $25^{\prime} 184$, ossia il $23.98 \%$, abitazioni locate a canone concordato risulta accessibile a questa famiglia mediana. La percentuale di sostenibilità risulta nettamente inferiore per il 2012 (9.18\%) e 2010 (6.54\%).

Poiché il $51.40 \%$ delle famiglie bolognesi appartiene a questa tipologia famigliare, è opportuno monitorare con attenzione questa situazione.

Fin qui è stata esaminata la sostenibilità degli affitti a canone concordato, ora ci si concentrerà sui valori di mercato. Complessivamente, poiché i canoni di mercato sono tendenzialmente superiori rispetto a quelli a canone concordato, il numero, e le 
relative percentuali, di abitazioni sostenibili sarà inferiore rispetto alle tabelle precedenti.

La Tabella 39 vuole essere la riproposizione della Tabella 36, con l'unica differenza che in questo caso la sostenibilità è definita per gli affitti a valori di mercato e non a canone concordato $^{58}$. La Tabella 40 riporta le percentuali di abitazioni sostenibili a canone di mercato, per zone e vani, quando la sostenibilità è definita in funzione del nucleo mediano di due persone, indipendentemente dal numero di vani dell'abitazione.

Tabella 39 - Percentuale di abitazioni sostenibili a valori di mercato, suddivise per zone di applicazione del canone concordato e numero di vani.

\begin{tabular}{|c|c|c|c|c|c|c|c|c|c|c|}
\hline \multicolumn{11}{|c|}{ Abitazioni sostenibili a canone di mercato } \\
\hline & & \multicolumn{3}{|c|}{2010} & \multicolumn{3}{|c|}{2012} & \multicolumn{3}{|c|}{2014} \\
\hline 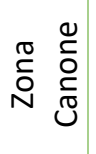 & 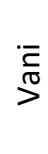 & ப் & 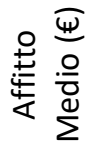 & 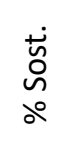 & ث் & 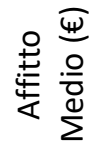 & 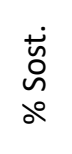 & ث் & 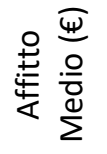 & 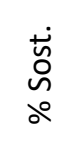 \\
\hline \multirow{6}{*}{ 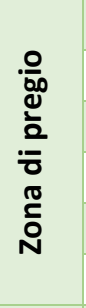 } & 1 & 468 & 447 & 100 & 536 & 443 & 100 & 628 & 386 & 100 \\
\hline & 2 & 287 & 596 & 63 & 318 & 588 & 79 & 368 & 513 & 100 \\
\hline & 3 & 624 & 770 & 59 & 640 & 759 & 58 & 767 & 659 & 93 \\
\hline & 4 & 560 & 964 & 2 & 576 & 951 & 2 & 686 & 823 & 26 \\
\hline & 5 & 437 & 1186 & 0 & 423 & 1169 & 0 & 517 & 1015 & 2 \\
\hline & 6 & 635 & 1724 & 0 & 626 & 1701 & 0 & 767 & 1475 & 0 \\
\hline \multirow{6}{*}{$\begin{array}{l}\varangle \\
\mathbb{0} \\
\check{c} \\
N\end{array}$} & 1 & 966 & 399 & 100 & 989 & 394 & 100 & 1165 & 345 & 100 \\
\hline & 2 & 864 & 513 & 93 & 860 & 509 & 96 & 1074 & 451 & 100 \\
\hline & 3 & 2491 & 646 & 92 & 2452 & 638 & 90 & 2999 & 575 & 95 \\
\hline & 4 & 1916 & 834 & 36 & 1838 & 822 & 35 & 2265 & 743 & 60 \\
\hline & 5 & 963 & 1050 & 6 & 929 & 1036 & 6 & 1154 & 928 & 27 \\
\hline & 6 & 820 & 1498 & 0 & 766 & 1467 & 0 & 907 & 1304 & 0 \\
\hline \multirow{6}{*}{$\begin{array}{l}\infty \\
0 \\
\check{c} \\
N\end{array}$} & 1 & 1203 & 316 & 98 & 1211 & 313 & 98 & 1469 & 282 & 98 \\
\hline & 2 & 1179 & 416 & 99 & 1188 & 411 & 99 & 1461 & 371 & 99 \\
\hline & 3 & 3716 & 530 & 100 & 3497 & 523 & 100 & 4406 & 474 & 100 \\
\hline & 4 & 2632 & 669 & 94 & 2534 & 663 & 93 & 3207 & 599 & 99 \\
\hline & 5 & 799 & 837 & 53 & 764 & 826 & 53 & 983 & 744 & 86 \\
\hline & 6 & 310 & 1147 & 5 & 304 & 1109 & 6 & 361 & 1013 & 19 \\
\hline
\end{tabular}

Come prevedibile, sia la Tabella 39 che la Tabella 40 evidenziano una minor sostenibilità per i canoni mensili medi di mercato rispetto a quelli definiti a canone

\footnotetext{
${ }^{58}$ La tabella è presentata solo con finalità di confronto con gli affitti potenziali a canone concordato. I valori di mercato vengono infatti definiti per aree omogenee e non per zone di applicazione del canone concordato. Poiché la sovrapposizione tra le due mappe non è perfetta, nonostante le abitazioni utilizzate siano perfettamente geolocalizzate, i canoni di mercato medi per zone di applicazione del canone concordato rappresentano una forzatura rispetto alla realtà.
} 
concordato, dettati dai maggiori affitti medi di questa tipologia contrattuale. Ancora una volta, per entrambe le tabelle, la percentuale di abitazioni sostenibili aumenta passando dallo stock 2010 a quello 2014. Le percentuali aumentano anche passando da Zona Pregio a Zona A e B, all'interno dello stesso anno, ma con un'intensità inferiore rispetto alle locazioni a canone concordato. Questo potrebbe essere dovuto al fatto che i prezzi di mercato sono calcolati per aree omogenee e non per zone di applicazione del canone concordato. Poiché non c'è una perfetta sovrapposizione tra le due definizioni, spesso un'area omogenea ricade in più di una zona del canone e influenza in questo modo $i$ canoni mensili medi utilizzati per definire la sostenibilità.

Tabella 40 - Percentuale di abitazioni sostenibili a valori di mercato per il nucleo mediano di due persone, suddivise per zone di applicazione del canone concordato e numero di vani.

\begin{tabular}{|c|c|c|c|c|c|c|c|c|c|c|}
\hline \multicolumn{11}{|c|}{ Abitazioni sostenibili a canone di mercato } \\
\hline & & \multicolumn{3}{|c|}{2010} & \multicolumn{3}{|c|}{2012} & \multicolumn{3}{|c|}{2014} \\
\hline 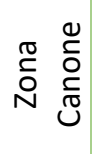 & $\begin{array}{l}\overline{\bar{E}} \\
\bar{\Gamma}\end{array}$ & ثீं & 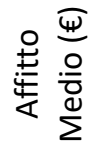 & $\begin{array}{l}\text { ते } \\
\text { ڤn } \\
\text { वீ}\end{array}$ & 苛 & 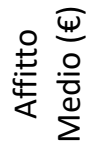 & $\begin{array}{l}\dot{n} \\
\text { ஸे } \\
\text { مீ}\end{array}$ & ث艹 & 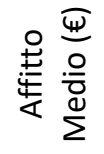 & 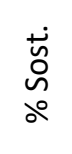 \\
\hline \multirow{6}{*}{$\begin{array}{l}\frac{0}{00} \\
\frac{0}{0} \\
\frac{0}{2} \\
\frac{0}{0} \\
\mathbb{0} \\
\stackrel{0}{0} \\
N\end{array}$} & 1 & 468 & 447 & 100 & 536 & 443 & 100 & 628 & 386 & 100 \\
\hline & 2 & 287 & 596 & 63 & 318 & 588 & 79 & 368 & 513 & 100 \\
\hline & 3 & 624 & 770 & 1 & 640 & 759 & 4 & 767 & 659 & 52 \\
\hline & 4 & 560 & 964 & 0 & 576 & 951 & 0 & 686 & 823 & 0 \\
\hline & 5 & 437 & 1186 & 0 & 423 & 1169 & 0 & 517 & 1015 & 0 \\
\hline & 6 & 635 & 1724 & 0 & 626 & 1701 & 0 & 767 & 1475 & 0 \\
\hline \multirow{6}{*}{ 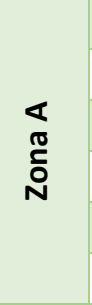 } & 1 & 966 & 399 & 100 & 989 & 394 & 100 & 1165 & 345 & 100 \\
\hline & 2 & 864 & 513 & 93 & 860 & 509 & 96 & 1074 & 451 & 100 \\
\hline & 3 & 2491 & 646 & 40 & 2452 & 638 & 53 & 2999 & 575 & 78 \\
\hline & 4 & 1916 & 834 & 2 & 1838 & 822 & 3 & 2265 & 743 & 31 \\
\hline & 5 & 963 & 1050 & 0 & 929 & 1036 & 0 & 1154 & 928 & 1 \\
\hline & 6 & 820 & 1498 & 0 & 766 & 1467 & 0 & 907 & 1304 & 0 \\
\hline \multirow{6}{*}{$\begin{array}{c}\infty \\
\mathbb{0} \\
\stackrel{0}{O} \\
N\end{array}$} & 1 & 1203 & 316 & 98 & 1211 & 313 & 98 & 1469 & 282 & 98 \\
\hline & 2 & 1179 & 416 & 99 & 1188 & 411 & 99 & 1461 & 371 & 99 \\
\hline & 3 & 3716 & 530 & 91 & 3497 & 523 & 96 & 4406 & 474 & 99 \\
\hline & 4 & 2632 & 669 & 25 & 2534 & 663 & 31 & 3207 & 599 & 82 \\
\hline & 5 & 799 & 837 & 1 & 764 & 826 & 2 & 983 & 744 & 18 \\
\hline & 6 & 310 & 1147 & 0 & 304 & 1109 & 0 & 361 & 1013 & 0 \\
\hline
\end{tabular}

\section{Concorrenzialità}

Il tema della sostenibilità affronta il problema dal punto di vista della domanda, quello della concorrenzialità si riferisce al lato dell'offerta. Si tratta di valutare, infatti, se il locatore possa avere convenienza ad applicare un contratto a canone concordato o 
meno. La sostenibilità, da sola, non è sufficiente per garantire l'accesso delle famiglie alle abitazioni: affinché avvenga una stipula del contratto è necessario che vi sia anche una soddisfazione delle condizioni poste dal conduttore. Quest'ultimo, infatti, potrebbe avere proposte migliori al di fuori del regime di canone concordato e decidere di non ricorrere a questa forma contrattuale. É definita "concorrenziale" un'abitazione il cui canone concordato medio al netto della cedolare secca (pari al $19 \%$ fino al 2013 , poi scesa al $10 \%$ dal 2014 ) è maggiore o uguale al canone medio a libero mercato, sempre al netto della cedolare secca al $21 \%$ prevista per questa tipologia di contratti.

La Tabella 41 presenta, per entrambi gli stock di abitazioni disponibili, il numero assoluto e le relative percentuali di alloggi che risultano concorrenziali a canone concordato, oltre che gli incassi medi netti per le due possibilità contrattuali, suddivise per zone di applicazione del canone concordato e numero di vani.

Tabella 41 - Percentuale di abitazioni concorrenziali a canone concordato, suddivise per zone di applicazione del canone concordato e numero di vani.

\begin{tabular}{|c|c|c|c|c|c|c|c|c|c|c|c|c|c|}
\hline \multicolumn{14}{|c|}{ Concorrenzialità abitazioni locate a canone concordato } \\
\hline & & \multicolumn{4}{|c|}{2010} & \multicolumn{4}{|c|}{2012} & \multicolumn{4}{|c|}{2014} \\
\hline $\begin{array}{l}0 \\
\stackrel{0}{0} \\
\text { N }\end{array}$ & $\begin{array}{l}\bar{c} \\
\text { 焉 }\end{array}$ & ثீं & 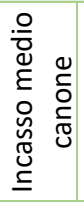 & 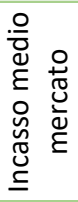 & 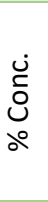 & 苍 & 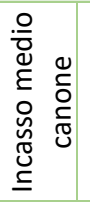 & 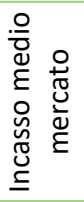 & $\begin{array}{l}\dot{\mathrm{c}} \\
\dot{0} \\
\text { ذo }\end{array}$ & 苍 & 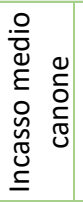 & 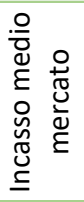 & $\begin{array}{l}\dot{\check{c}} \\
\text { ठ̀ } \\
\text { ó }\end{array}$ \\
\hline \multirow{6}{*}{$\begin{array}{l}\frac{0}{00} \\
\frac{0}{0} \\
\frac{2}{2} \\
\frac{0}{0} \\
\frac{1}{0} \\
\frac{1}{0}\end{array}$} & 1 & 468 & 365 & 353 & 46 & 536 & 365 & 350 & 46 & 628 & 374 & 305 & 100 \\
\hline & 2 & 287 & 426 & 471 & 5 & 318 & 428 & 464 & 5 & 368 & 439 & 406 & 79 \\
\hline & 3 & 624 & 478 & 608 & 0 & 640 & 476 & 599 & 0 & 767 & 490 & 521 & 33 \\
\hline & 4 & 560 & 558 & 761 & 0 & 576 & 558 & 751 & 0 & 686 & 574 & 650 & 0 \\
\hline & 5 & 437 & 612 & 937 & 0 & 423 & 611 & 923 & 0 & 517 & 632 & 802 & 0 \\
\hline & 6 & 635 & 797 & 1362 & 0 & 626 & 798 & 1344 & 0 & 767 & 822 & 1165 & 0 \\
\hline \multirow{6}{*}{ 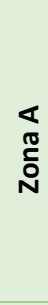 } & 1 & 966 & 311 & 315 & 41 & 989 & 309 & 311 & 40 & 1165 & 318 & 273 & 91 \\
\hline & 2 & 864 & 352 & 405 & 10 & 860 & 351 & 402 & 10 & 1074 & 361 & 356 & 58 \\
\hline & 3 & 2491 & 389 & 510 & 0 & 2452 & 389 & 504 & 0 & 2999 & 401 & 454 & 29 \\
\hline & 4 & 1916 & 459 & 659 & 0 & 1838 & 459 & 649 & 0 & 2265 & 473 & 587 & 6 \\
\hline & 5 & 963 & 507 & 830 & 0 & 929 & 506 & 819 & 0 & 1154 & 519 & 733 & 0 \\
\hline & 6 & 820 & 624 & 1183 & 0 & 766 & 621 & 1159 & 0 & 907 & 633 & 1031 & 0 \\
\hline \multirow{6}{*}{ 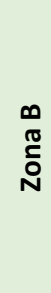 } & 1 & 1203 & 267 & 250 & 71 & 1211 & 266 & 247 & 71 & 1469 & 268 & 223 & 96 \\
\hline & 2 & 1179 & 304 & 329 & 10 & 1188 & 303 & 325 & 10 & 1461 & 306 & 293 & 58 \\
\hline & 3 & 3716 & 339 & 419 & 0 & 3497 & 338 & 414 & 11 & 4406 & 340 & 375 & 3 \\
\hline & 4 & 2632 & 391 & 529 & 0 & 2534 & 391 & 524 & 0 & 3207 & 395 & 473 & 0 \\
\hline & 5 & 799 & 430 & 661 & 0 & 764 & 431 & 653 & 0 & 983 & 434 & 588 & 0 \\
\hline & 6 & 310 & 542 & 906 & 0 & 304 & 529 & 876 & 0 & 361 & 542 & 800 & 0 \\
\hline
\end{tabular}

Osservando i valori netti medi di incasso, per il 2010 e il 2012, indipendentemente dalla zona di applicazione del canone concordato, l'unica tipologia abitativa che risulta 
conveniente per il proprietario, sulla base di un mero confronto tra i valori medi, è il monolocale. Nel 2014, grazie alla riduzione dell'aliquota applicata al contratto di canone concordato, indipendentemente dalla zona, i valori medi netti di incasso del canone concordato risultano superiori a quelli di mercato anche per i bilocali oltre che per i monolocali.

Per tutte le abitazioni con un numero maggiore di vani, il mercato assicura un valore medio di incasso superiore, con una differenza molto marcata nel caso delle abitazioni con più locali, rispetto a quello di canone concordato.

Non essendo intervenute modifiche legislative tra il 2010 e il 2012, l'unica differenza tra questi due anni, oltre ai rispettivi prezzi di locazione a canone di mercato e a canone concordato, è lo stock di abitazioni utilizzato come base campionaria. Per questa coppia di anni un'abitazione è definita concorrenziale se il canone concordato al netto della cedolare secca al $19 \%$ assicura al proprietario dell'immobile un'entrata non inferiore al canone di mercato scontato della rispettiva cedolare secca (21\%).

Essendo I'unica differenza determinata dai prezzi e dallo stock di abitazioni, le percentuali di alloggi concorrenziali per zone e numero di vani risulta simile tra i due anni. Dato il basso scarto percentuale tra le due aliquote, il numero di abitazioni concorrenziali è relativamente basso e in generale le uniche abitazioni che risultano in parte concorrenziali sono quelle di piccola metratura.

Nel 2014 la cedolare secca da applicare ai contratti a canone concordato scende al $10 \%$, aumentando il differenziale rispetto all'aliquota di mercato, e conseguentemente aumenta il numero di abitazioni concorrenziali. Questo sconto fiscale va a bilanciare la riduzione dei canoni mensili e fa sì che la percentuale di affitti concorrenziali aumenti rispetto agli anni precedentemente considerati. Nonostante il miglioramento in termini di disponibilità di offerta di locazione a canone concordato, la quasi totalità degli alloggi con un numero di vani superiore a tre continua a risultare non concorrenziale. Questo di per sé non indica che le abitazioni non siano a canone concordato, anzi, come sappiamo circa 25'000 unità sono attualmente locate a canone concordato. Cosa sta avvenendo quindi? Semplicemente i prezzi di mercato $\mathrm{OMI}$ essendo una media delle medie, per quanto sottostimino il reale valore degli affitti a mercato libero, permettono di intuire quale meccanismo porti verso il canone concordato:

- Una vasta maggioranza di famiglie potrebbe trovare non sostenibili i canoni di mercato;

- La gestione di un canone di affitto libero è maggiormente onerosa ed essa riduce quindi l'appetibilità degli stessi da parte dei conduttori;

- La cedolare secca al $10 \%$ drasticamente riduce la tassazione diventando un importante scudo che viene probabilmente percepito come più sicuro rispetto alla cedolare secca per gli affitti a canone libero.

- La domanda di locazione a Bologna è particolarmente sostenuta ma la sua forte componente studentesca genera meccanismi di rapido turnover che 
rende profittevole mantenere elevata la presenza di affittuari nelle unità abitative piuttosto che massimizzare i prezzi essendo comunque il margine di profitto elevato.

\section{Domanda e offerta di locazione a canone concordato}

Concorrenzialità e sostenibilità non sono, considerate singolarmente, condizioni sufficienti per definire il numero effettivo di alloggi affittabili mediante canone concordato. In questo paragrafo si vogliono esaminare congiuntamente queste due condizioni per capire quante delle abitazioni del dataset in esame siano effettivamente affittabili mediante questa tipologia contrattuale.

La combinazione delle due variabili (concorrenzialità e sostenibilità) genera quattro possibili combinazioni. Una di queste (sostenibile, non concorrenziale) è stata ulteriormente suddivisa per tenere conto anche della sostenibilità a mercato. Vengono distinte cinque categorie in cui collocare le abitazioni a seconda della combinazione tra sostenibilità e concorrenzialità:

- Non sostenibile, non concorrenziale;

- Sostenibile, concorrenziale;

- Non sostenibile, non concorrenziale, non sostenibile a canone di mercato;

- Non sostenibile, non concorrenziale, sostenibile a canone di mercato;

- Non sostenibile, concorrenziale.

La Figura 41 rappresenta, sul totale degli alloggi disponibili anno per anno, la distribuzione percentuale delle abitazioni nelle diverse categorie individuate.

Nessuna delle abitazioni presenti nel dataset è riconducibile alla categoria "Non sostenibile - Concorrenziale". La quota di contratti "Non sostenibili - Non concorrenziali" è sempre contenuta tra il $2 \%$ e il $3 \%$, indipendentemente dall'anno di riferimento. La percentuale di maggior interesse è quella relativa agli alloggi effettivamente collocabili mediante contratto di locazione a canone concordato: "Sostenibili - Concorrenziali". Con riferimento a questo gruppo la situazione sembra migliorare progressivamente nel tempo: le abitazioni riconducibili a questa categoria sono l'8.14\% dello stock 2010, il 10.44\% dello stock 2012 e, grazie alla riduzione della cedolare secca occorsa a partire dal 2014, il $24.97 \%$ per l'ultimo anno disponibile. Le restanti categorie fanno riferimento alla combinazione "Sostenibile - Non concorrenziale", che viene ulteriormente suddivisa in base alla sostenibilità del mercato libero. Ovviamente la categoria "Sostenibile - Non concorrenziale Sostenibile a mercato" (54.58\% nel 2014 ) appare come caso in cui la domanda ottiene un surplus positivo dal canone concordato stesso. A canone di mercato, infatti, tali abitazioni sarebbero sostenibili per le famiglie che, invece, a canone concordato riescono ad ottenere minor esborso. In quest'ottica il canone concordato sta assolvendo alla sua funzione di calmierare i prezzi favorendo il benessere delle 
famiglie. Ancora più positivo l'effetto del canone concordato per quelle abitazioni che appartengono alla categoria "Sostenibile - Non concorrenziale - Non sostenibile a mercato". Esse, infatti, non otterrebbero soddisfazione (non sarebbero locate) ai prezzi di mercato. Si ricorda comunque che tali valutazioni sono effettuate sulla base della famiglia mediana, ergo l'analisi non sostiene che non vi possano essere sul mercato soggetti che hanno le disponibilità economiche per sostenere i prezzi a libero mercato.

Figura 41. Distribuzione abitazioni per sostenibilità e concorrenzialità, 2010-2012-2014, Bologna.

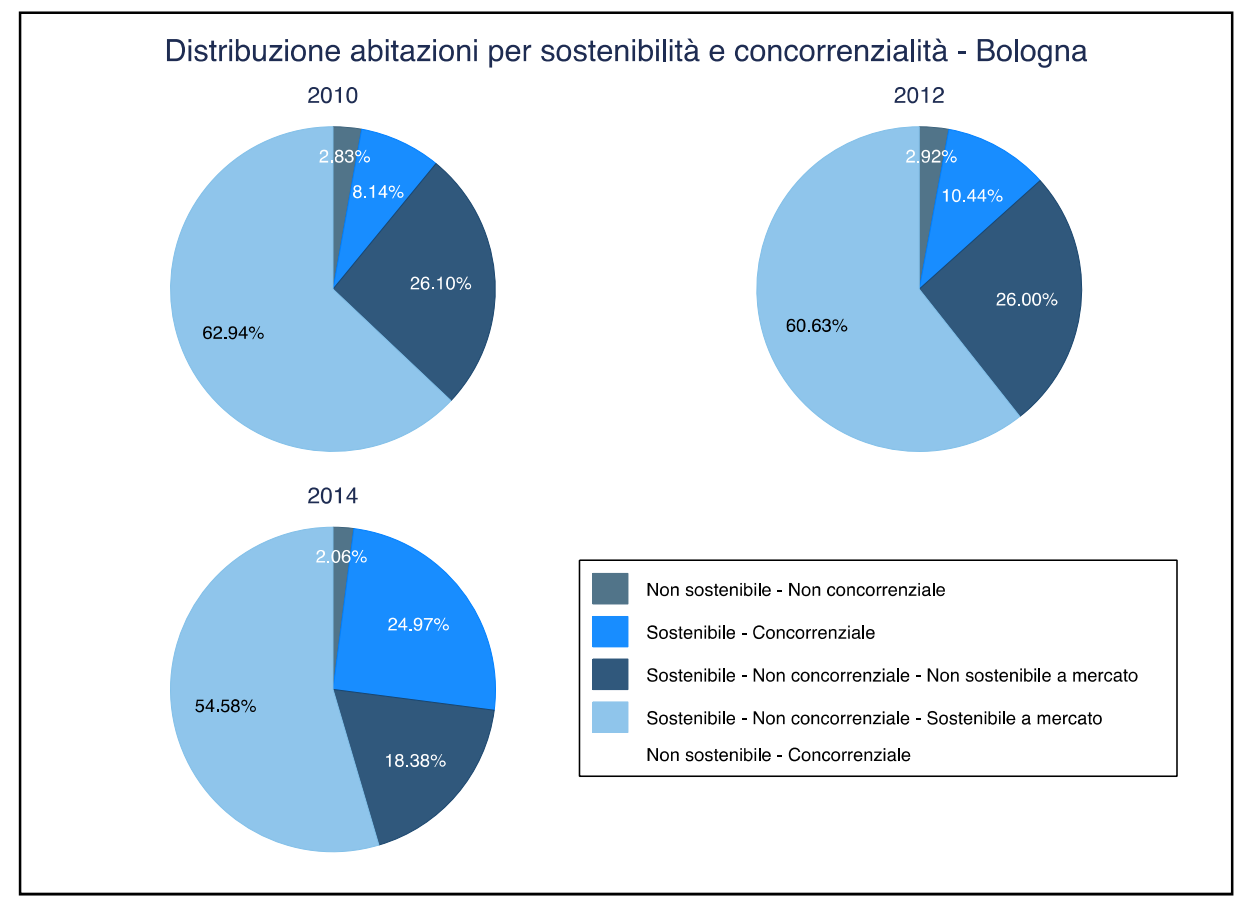

La Tabella 42 presenta una serie di statistiche descrittive per alcune variabili (numero di abitazioni, superficie media, numero medio di vani, canone concordato mensile medio, incidenza media del canone sulla soglia e canone di mercato mensile medio) suddivise nelle categorie precedentemente identificate. L'ultima colonna della Tabella 42 presenta, anno per anno, i valori medi delle variabili riportate relativamente all'intero stock di alloggi a disposizione. Considerando inizialmente i valori medi anno per anno, indipendentemente dalla suddivisione nelle categorie precedentemente individuate, non si notano grandi differenze per quanto riguarda la superficie e il numero medio di vani ( $79.79 \mathrm{mq}$ e 3.32 vani sono i valori medi 2014). Al contrario, nel 2014 si assiste ad un'importante riduzione del canone concordato mensile, e di conseguenza anche dell'incidenza dello stesso sulla soglia di sostenibilità familiare. Se nel 2012, infatti, si registra un valore medio di 507.93€ mensili ed un'incidenza media dell' $81 \%$, nel 2014 il canone mensile medio si attesta a $465.72 €$ (riduzione dell' $8.31 \%$ rispetto al valore 2012), ossia $71 \%$ della soglia di sostenibilità familiare. 
Tabella 42 - Distribuzione alloggi per categorie di sostenibilità e concorrenzialità e relative statistiche descrittive.

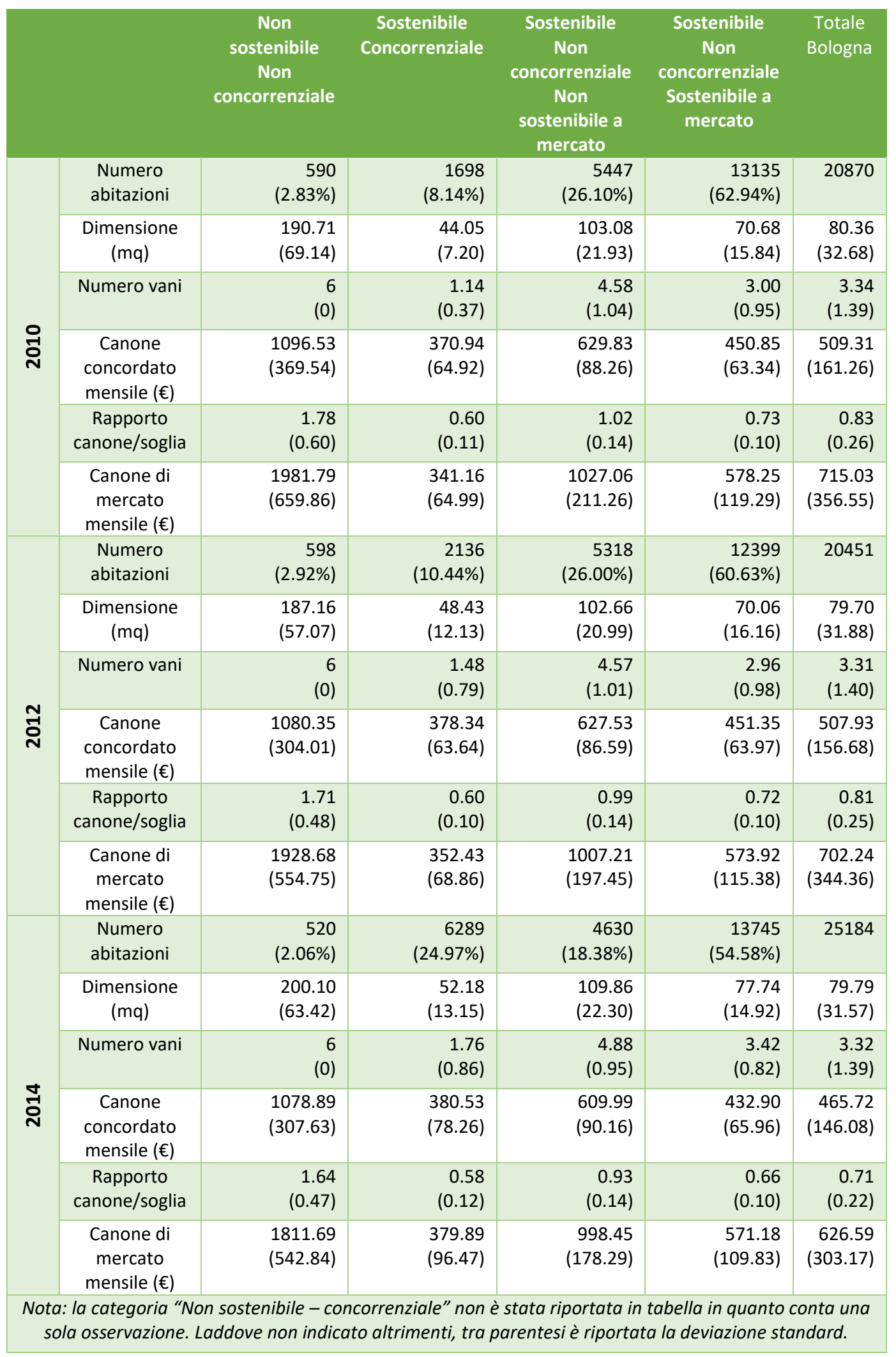


Poiché esiste un'importante correlazione tra i prezzi del canone concordato e del canone di mercato, nel 2014 si assiste anche ad una riduzione del canone di mercato mensile: $702.24 €$ nel 2012 e $626.59 €$ nel 2014 , segnando una riduzione del $10.77 \%$ tra i due anni.

Approfondendo l'analisi alle singole categorie evidenziate, ciò che è interessante notare è la distribuzione del numero di vani e delle superfici all'interno delle stesse. Tutte gli alloggi "Non sostenibili - Non concorrenziali" sono abitazioni di sei locali, con canoni d'affitto molto alti e tassi di incidenza dell'affitto sulla soglia ben superiori all'unità, che indicano come la sostenibilità sia lontana dall'essere raggiunta. Diversamente le abitazioni "Sostenibili - Concorrenziali" sembrano essere quelle più piccole: la superficie media varia dai $44.05 \mathrm{mq}$ del 2010 ai $52.18 \mathrm{mq}$ del 2014, con un numero di vani medio pari a 1.14 per il primo anno e a 1.76 per l'ultimo.

Più eterogenei sono gli alloggi appartenenti alle ultime due categorie (escludendo quella delle abitazioni "Non sostenibili - Concorrenziali", per cui non si registrano osservazioni), come dimostrano anche le deviazioni standard maggiori riportate in corrispondenza delle loro statistiche descrittive.

In conclusione, è opportuno sottolineare come, nonostante l'incremento del numero di abitazioni che soddisfano domanda e offerta di locazione nel corso degli anni, solamente un'abitazione su quattro nel 2014 è teoricamente locabile tramite contratto a canone concordato. Inoltre, si tratta di abitazioni molto piccole in termini di metratura e numero di vani, tendenzialmente inadatte a famiglie di due o più componenti. Le due categorie riconducibili alla casistica "Sostenibile - Non concorrenziale" racchiudono la maggior parte delle abitazioni, nonché quelle di maggior interesse per le famiglie bolognesi (dimensioni medie). Queste abitazioni (72.96\% dello stock 2014 ) soddisfano le esigenze degli inquilini (sostenibilità), ma non risultano concorrenziali a canone concordato per i proprietari, che hanno una maggior convenienza a locarle tramite canone di mercato.

Concorrenzialità e sostenibilità all'interno delle zone di applicazione del canone concordato

Fin qui l'analisi della sostenibilità e concorrenzialità si è concentrata sulle abitazioni locale a canone concordato nel comune di Bologna, considerato nel suo insieme. Un maggior livello di dettaglio può essere ottenuto studiando aree più ristrette della città, in quanto gli elementi trattati potrebbero non distribuirsi uniformemente su tutto il territorio. Si procederà ora a presentare la stessa analisi per le tre aree di applicazione del canone concordato (Zona di Pregio, Zona A, Zona B). 
La Figura 42 individua la Zona di Pregio sul territorio cittadino. Questa presenta i prezzi di locazione a canone concordato, e quindi anche di mercato, maggiori e coincide con la zona collinare e la parte centro-meridionale del centro storico. In quest'area trovano collocazione il $14.35 \%$ (3012), il $15.15 \%$ (3120) e il $14.82 \%$ (3733) degli stock di abitazioni 2010, 2012 e 2014. La Figura 43 rappresenta la distribuzione di questi alloggi tra le categorie precedentemente evidenziate dalla combinazione tra sostenibilità e concorrenzialità, mentre la Tabella 43 riporta le statistiche descrittive delle abitazioni appartenenti a questa zona di applicazione del canone concordato, per i tre anni relativi agli stock di abitazioni disponibili.

Figura 42 - Confini della zona di pregio sul territorio cittadino.

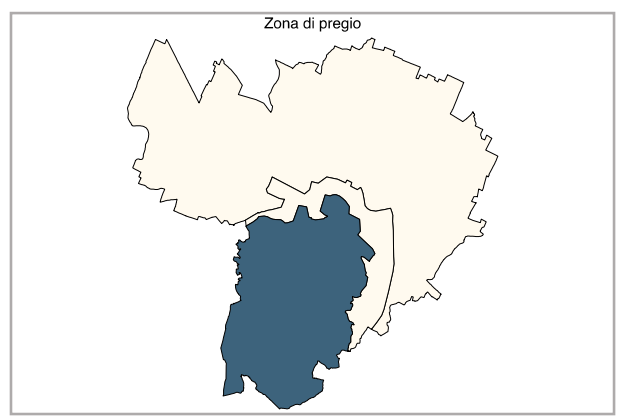

Figura 43. Distribuzione abitazioni per sostenibilità e concorrenzialità, 2010-2012-2014, Zona di pregio.

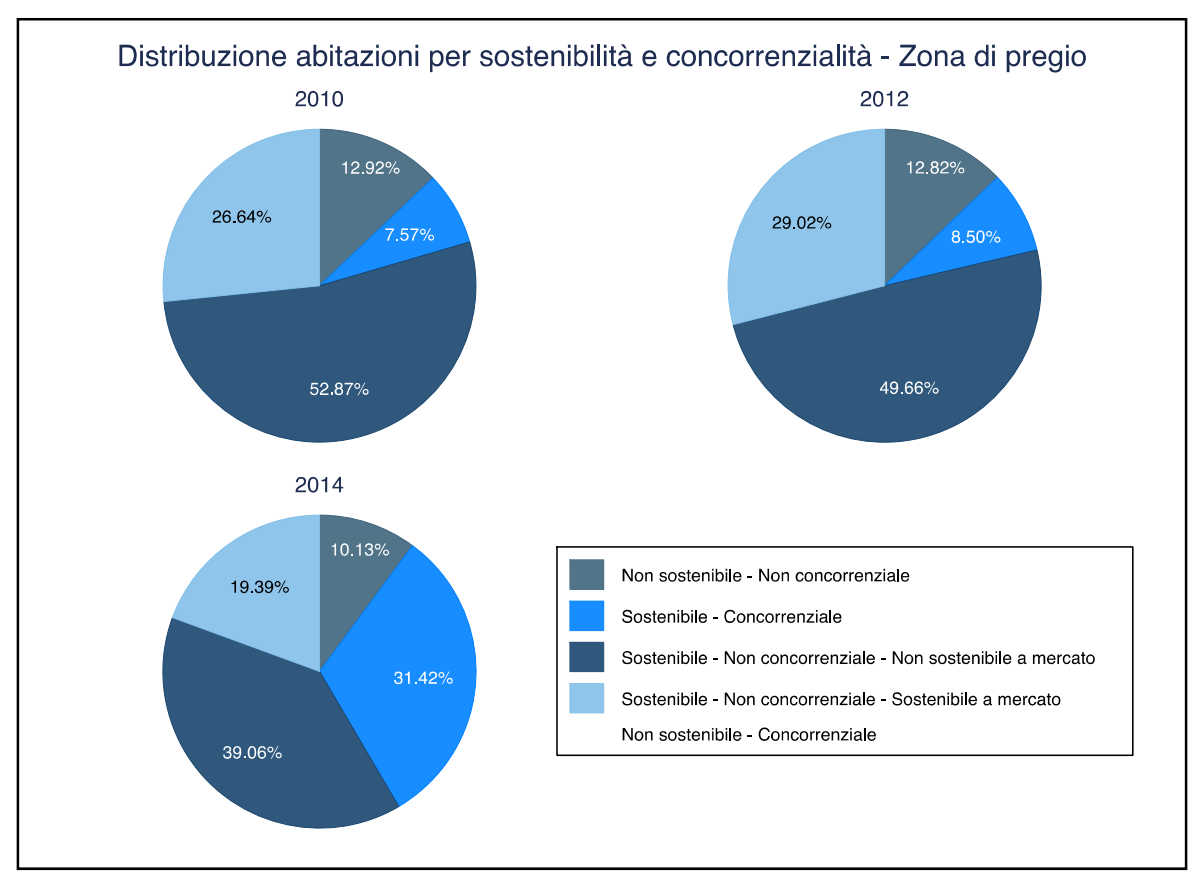


Tabella 43. Distribuzione alloggi per categorie di sostenibilità e concorrenzialità e relative statistiche descrittive.

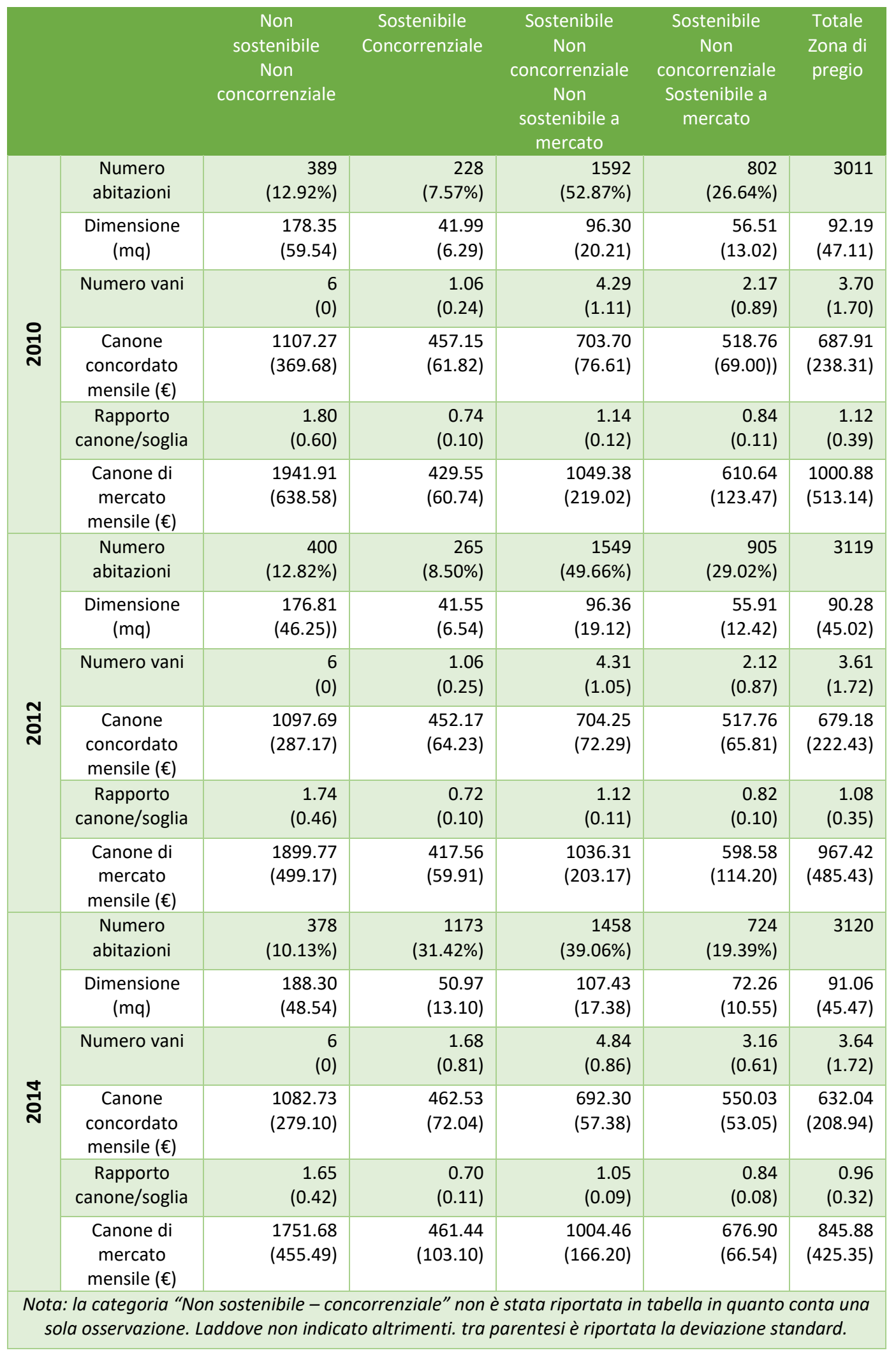


La percentuale di abitazioni "Non sostenibili - Non concorrenziali" della zona di pregio è nettamente superiore alla media cittadina, con valori osservati tra il $2 \%$ e il $3 \%$ per tutti gli anni. La riduzione dei prezzi al metro quadro per gli affitti a canone concordato, definita nell' accordo 2013, provoca un aumento consistente nella quota di abitazioni sia sostenibili che concorrenziali: 8.50\% nel 2012 e 31.42\% nel 2014 . Questo aumento è verificabile anche quando si considera il comune di Bologna nel suo complesso, ma in quel caso l'intensità è inferiore, indicando quindi un maggior beneficio, derivante dalla riduzione dei valori di locazione, per la Zona di Pregio rispetto alla città nel suo complesso. Nonostante questa nota positiva, però, la quota di abitazioni non sostenibili né a canone concordato né a mercato è molto alta (più della metà degli alloggi totali nel 2010), anche nel 2014 il valore registrato (39.06\%) è alto, seppur nettamente inferiore rispetto agli anni precedenti.

Confrontando le statistiche descrittive tra i diversi anni riportati si riscontra una netta differenza tra i primi due anni e il 2014. Nonostante gli stock di abitazioni applicati ai valori 2010 e 2012 siano diversi, la dimensione media delle abitazioni, il numero di vani, il canone d'affitto medio a canone concordato e a mercato e l'incidenza del canone mensile sulla soglia di sostenibilità sono molto simili. È interessante notare come, a livello complessivo per la Zona di Pregio, il valore medio del rapporto tra canone e soglia sia inferiore all'unità solamente nel 2014, anche se il 96\% rappresenta una quota molto vicina al limite massimo di spesa.

Zona A

La Figura 44 evidenzia la Zona A sul territorio cittadino. Le due sezioni distaccate rispetto all'area più grande sono incluse nella Zona $B$, ma a queste abitazioni si applicano i valori del canone concordato della Zona A, ed abbiamo pertanto deciso di inserirle nell'analisi relativa a quest'area. Questa presenta i prezzi di locazione a canone concordato intermedi rispetto alle altre due aree e coincide con la parte settentrionale del centro storico e le aree appena fuori dalla cinta muraria, escluse quelle a Sud. In quest'area trovano collocazione il 38.43\% (8'020), il 38.31\% (7' 834 ) e il 37.98\% (9'564) degli stock di alloggi 2010, 2012 e 2014.

La Figura 45 rappresenta la distribuzione di questi alloggi tra le categorie precedentemente evidenziate dalla combinazione tra sostenibilità e concorrenzialità, mentre la Tabella 44 riporta le statistiche descrittive delle abitazioni appartenenti a questa zona di applicazione del canone concordato, per i tre anni relativi agli stock di alloggi disponibili.

La percentuale di abitazioni "Non sostenibili - Non concorrenziali" della zona A è allineata alla media cittadina e in generale molto contenuta, per tutti gli anni osservati. La riduzione dei prezzi al metro quadro per gli affitti a canone concordato, definita nell'accordo 2013, unitamente alla riduzione della cedolare secca al $10 \%$, 
provoca un aumento consistente nella quota di abitazioni sia sostenibili che concorrenziali: $6.24 \%$ nel 2012 e $28.25 \%$ nel 2014 . Questo aumento è verificabile anche quando si considera il Comune di Bologna nel suo complesso, ma in quel caso l'intensità è inferiore, indicando quindi un maggior beneficio, derivante dalla riduzione dei valori di locazione, per la zona A rispetto alla città nel suo complesso. Nonostante questa nota positiva, però, la quota di abitazioni non sostenibili né a canone concordato né a mercato è superiore alla media cittadina, ma comunque inferiore rispetto ai valori registrati per la zona di pregio.

Figura 44 - Confini della zona A sul territorio cittadino.

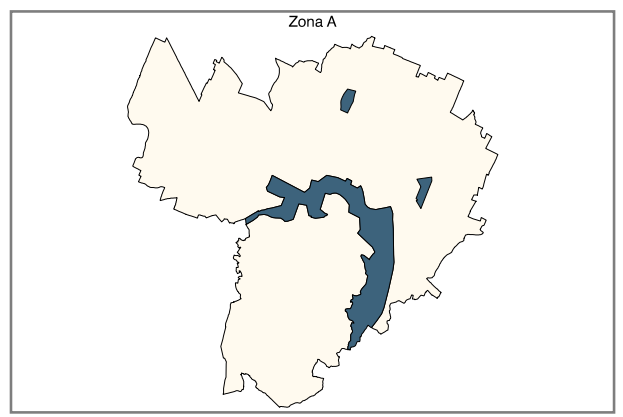

Figura 45. Distribuzione abitazioni per sostenibilità e concorrenzialità, 2010-2012-2014, Zona A.

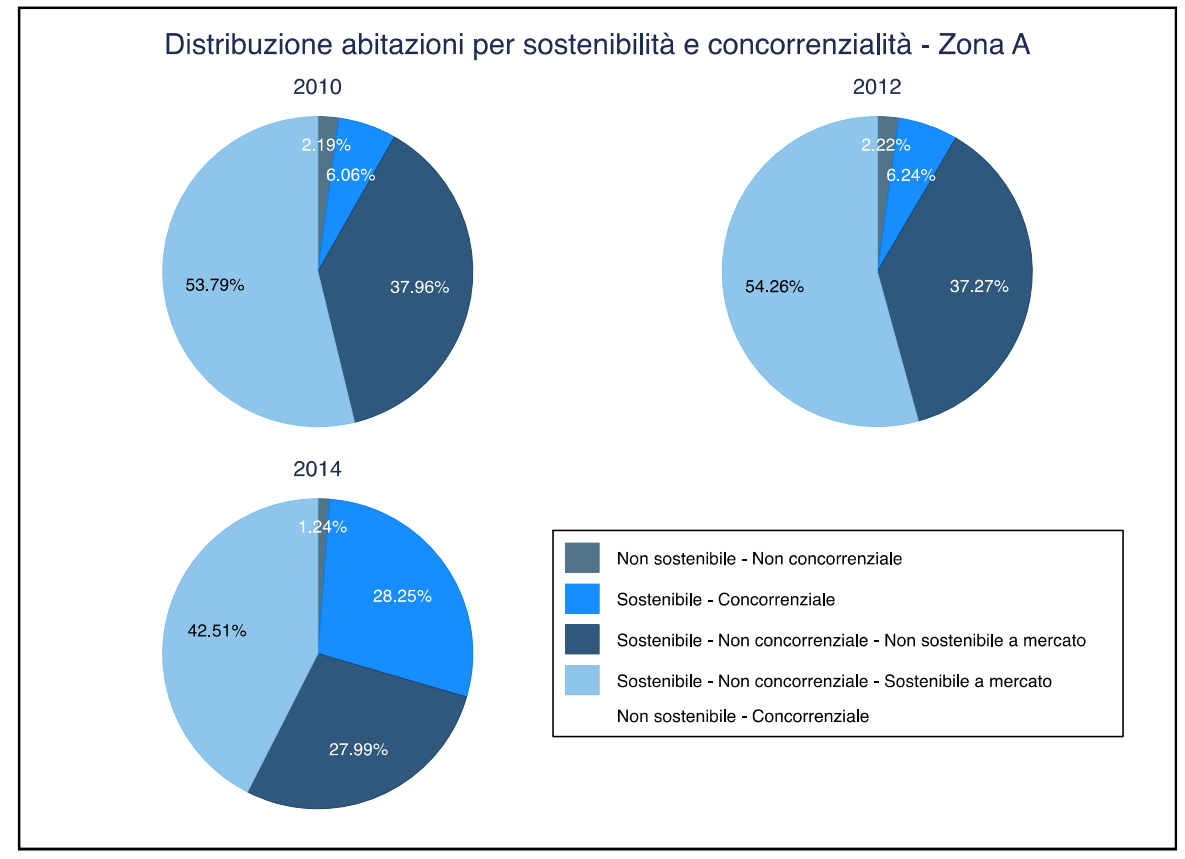


Tabella 44. Distribuzione alloggi per categorie di sostenibilità e concorrenzialità e relative statistiche descrittive.

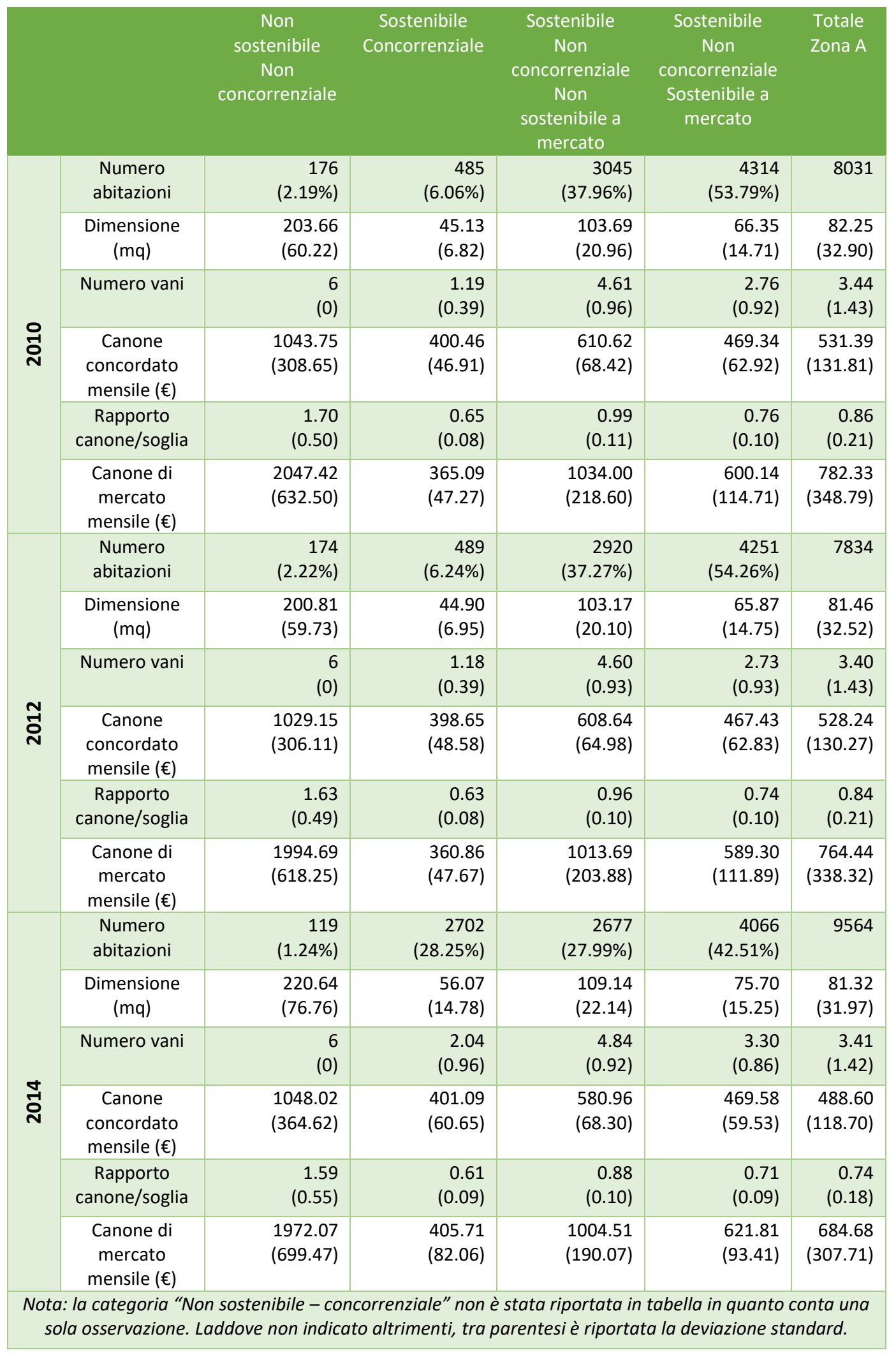


Come per la zona di pregio persiste una certa stabilità tra le statistiche descrittive (Tabella 44) all'interno delle singole categorie tra gli anni 2010 e 2012. Diversi sono invece i valori di riferimento per il 2014, a causa della diversa classificazione delle abitazioni all'interno delle categorie, dovuta all' approvazione del più recente accordo di applicazione del canone concordato. A livello complessivo (ultima colonna della Tabella 44) i canoni mensili risultano inferiori rispetto alla zona di pregio, e per tutti gli anni considerati il livello di incidenza del canone sulla soglia di sostenibilità è inferiore all'unità, rendendo in media sostenibile l'affitto in quest'area.

\section{Zona B}

La Figura 46 vuole evidenziare l'area di riferimento della Zona B sul territorio cittadino. Questa presenta i prezzi di locazione a canone concordato più bassi e coincide con l'area periferica settentrionale.

Figura 46 - Confini della zona B sul territorio cittadino.

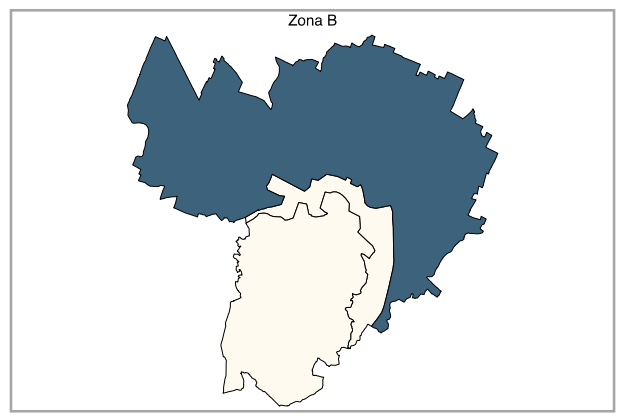

Figura 47. Distribuzione abitazioni per sostenibilità e concorrenzialità, 2010-2012-2014, Zona B.

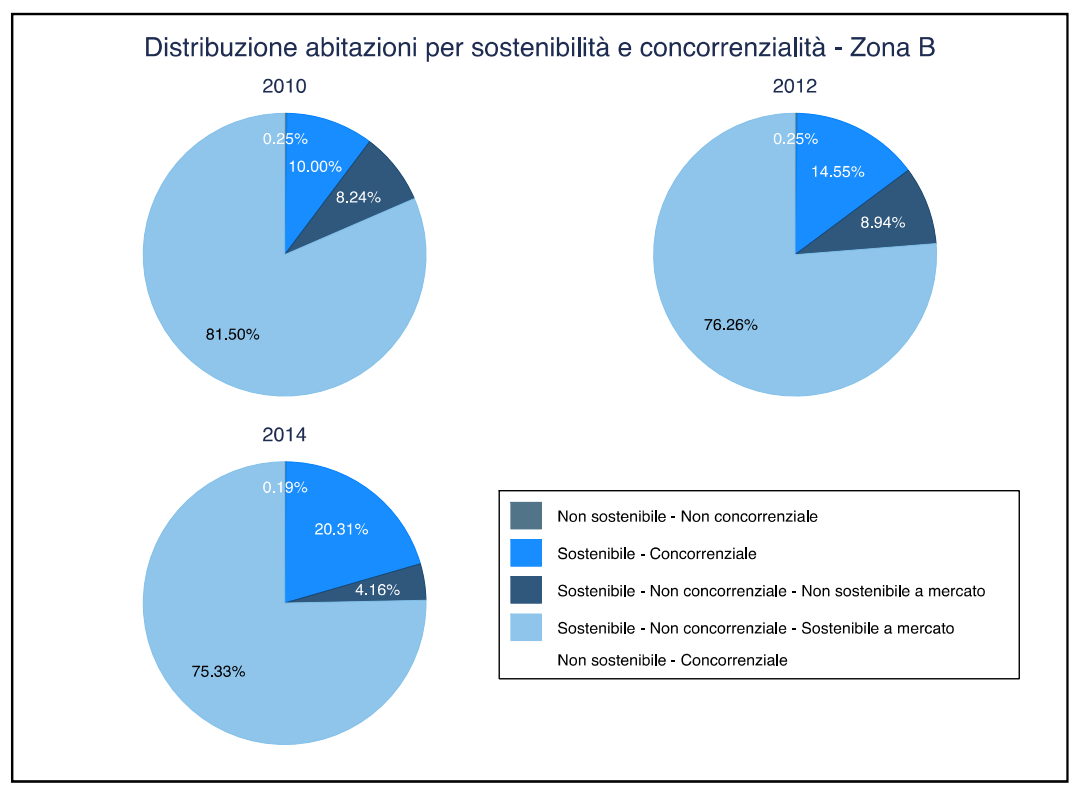


Tabella 45. Distribuzione alloggi per categorie di sostenibilità e concorrenzialità e relative statistiche descrittive.

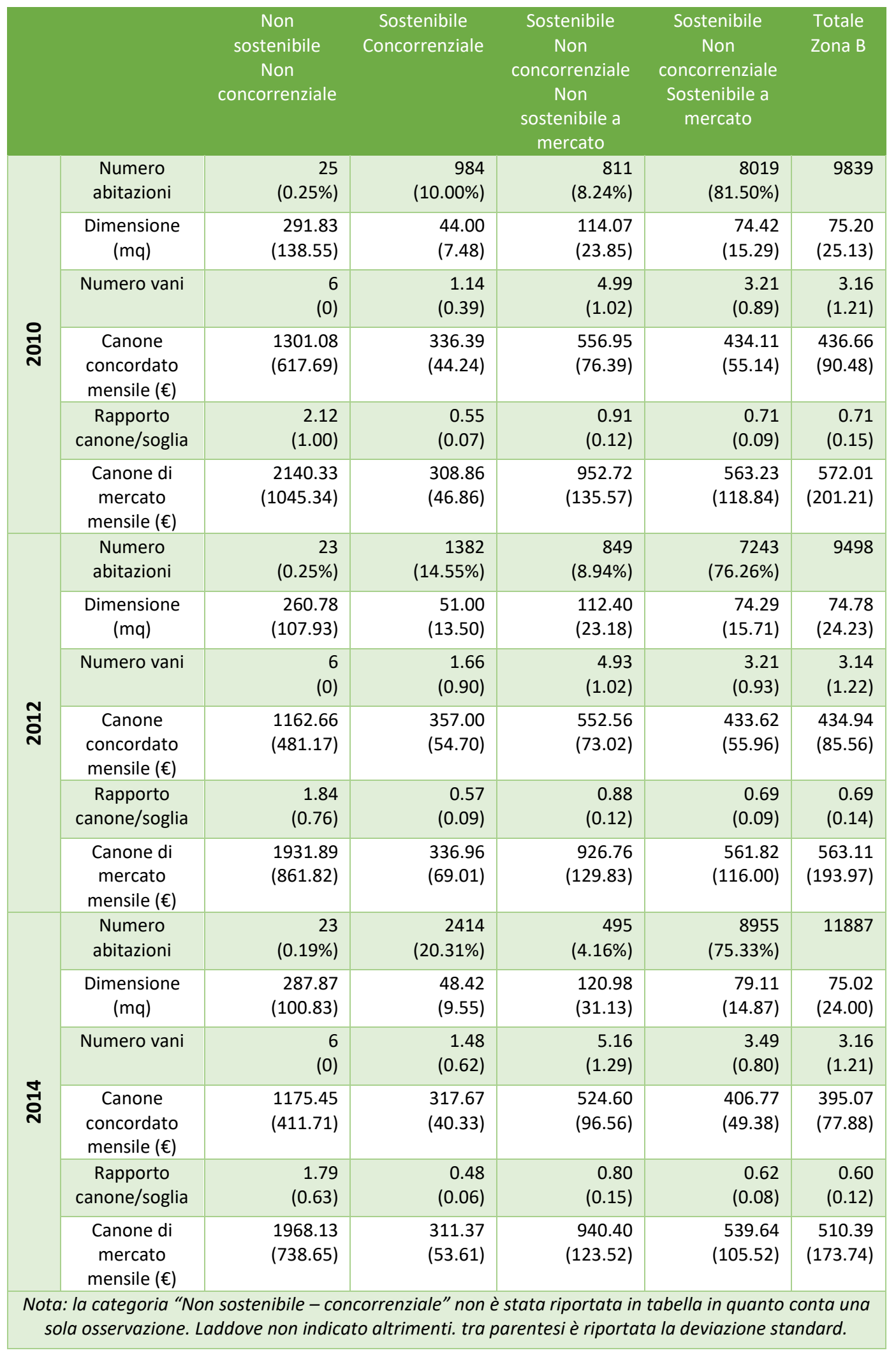


In quest'area trovano collocazione la maggior parte delle abitazioni degli stock disponibili: il 47.14\% (9'839), il 46.44\% (9'498) e il 47.20\% (11'887), rispettivamente per il 2010, 2012 e 2014. La Figura 47 rappresenta la distribuzione di queste abitazioni tra le categorie precedentemente evidenziate dalla combinazione tra sostenibilità e concorrenzialità, mentre la Tabella 45 riporta le statistiche descrittive delle abitazioni appartenenti a questa zona di applicazione del canone concordato, per i tre anni relativi agli stock di alloggi disponibili. È pressoché nulla la quota di abitazioni "Non sostenibili - Non concorrenziali" della Zona B, e quindi inferiore rispetto al valor medio cittadino (leggermente inferiore al 3\%).

Molto alta risulta la percentuale di alloggi non concorrenziali e sostenibili sia a canone concordato che a mercato: in questa categoria ricadono 3 alloggi su quattro nell'ultimo anno osservato. La quota di abitazioni migliori dal punto di vista di incontro tra domanda e offerta, ossia quelle sostenibili e concorrenziali, aumenta quasi analogamente tra i primi due anni e gli ultimi due. Nonostante questo, la quota relativa a questa categoria risulta nel 2014 più bassa rispetto alla media cittadina e alle percentuali registrate nelle altre zone $(20.31 \%$ nella zona B, $24.97 \%$ la media cittadina e $\mathbf{2 8 . 2 5 \%}$ e $31.42 \%$ rispettivamente per Zona A e di pregio). L'aumento del numero di abitazioni concorrenziali e sostenibili tra il 2012 e il 2014 indica come l'accordo 2013 abbia avuto effetti positivi in quest'area, ma i maggiori aumenti registrati in Zona A e di pregio segnalano come i benefici siano stati, seppur presenti, inferiori per quest'area.

La Tabella 45 presenta per la zona B una maggiore variabilità temporale delle statistiche descrittive relative ad ogni categoria, anche tra il 2010 e 2012 rispetto a quanto riscontrato per le altre aree della città. Questo è dovuto alla modifica graduale delle abitazioni componenti le diverse categorie sostenibilità-concorrenzialità, diversamente dalle altre zone in cui la modifica era molto più netta tra 2012 e 2014, senza osservare grandi differenze tra i primi due anni.

\section{Concorrenzialità e sostenibilità all'interno delle zone OMI}

Dopo aver analizzato la diversa distribuzione delle abitazioni secondo le possibili combinazioni di concorrenzialità e sostenibilità all'interno delle zone di applicazione del canone concordato, si procede ora a riportare, in modo più sintetico, l'analisi relativa alle zone OMI. In Appendice online sono riportati i valori specifici per ogni zona OMI mentre in testo sono riportate le mappe di riferimento.

Le diverse gradazioni di colore nelle mappe della Figura 48 fanno riferimento alle percentuali di abitazioni sostenibili e concorrenziali, riportate nelle tabelle precedenti. Si è deciso di rappresentare sinteticamente mediante questa figura solamente questa tipologia di abitazioni poiché si tratta di quelle che soddisfano contemporaneamente domanda e offerta. Si tratta, secondo le definizioni adottate in questa analisi, delle abitazioni effettivamente allocabili a canone concordato. 
A livello complessivo è possibile osservare un generale aumento di questa tipologia di abitazioni tra gli anni considerati, come testimonia la maggior presenza di tonalità scure nelle mappe 2012 e 2014 rispetto alla prima.

Concentrandosi sulle zone del centro città, l'incremento delle percentuali è netto: si passa dallo $0 \%$ di abitazioni sostenibili e concorrenziali nel 2010 e 2012 per quasi tutte le zone OMI presenti in quest'area, a percentuali comprese tra il $10-20 \%$ e il $20-50 \%$ nel 2014. Questo netto incremento, ben visibile per il centro storico ma presente anche nelle zone periferiche, è probabilmente determinato da una coppia di fattori.

Figura 48. Percentuali di abitazioni sostenibili e concorrenziali per zona OMI, Comune di Bologna.

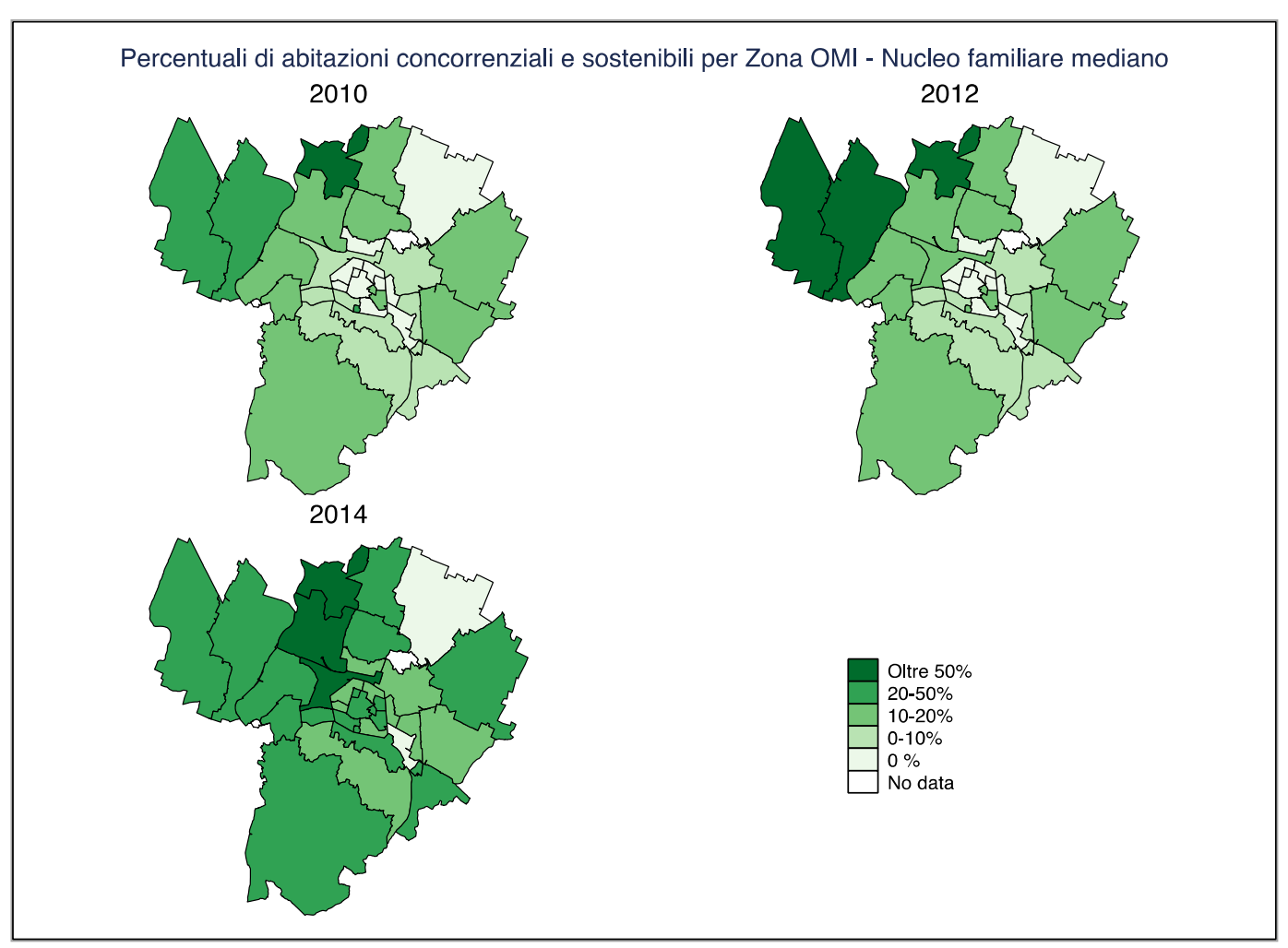

Da un lato la riduzione dei prezzi di locazione a canone concordato, definiti dall'accordo 2013, ha reso più sostenibili le abitazioni; dall'altro, lo sconto fiscale identificabile nella riduzione della cedolare secca al $10 \%$ per i contratti a canone concordato - anch'esso in vigore dal 2014, rappresenta un fattore di incremento della concorrenzialità del canone concordato rispetto al mercato per i proprietari.

La zona R2 ("Agricola Nord") è l'unica a presentare una percentuale appartenente alla fascia più alta per tutti gli anni considerati: l'84\% delle abitazioni di questa zona dello stock 2010 risultano sostenibili e concorrenziali, la percentuale è maggiore del 90\% per gli ultimi due anni. È importante sottolineare, però, come questa zona abbia un peso marginale sul campione totale in termini di numero di abitazioni. 
Interessante è anche il caso della zona D24 ("Murri"), l'unica a presentare lo $0 \%$ di abitazioni di questa tipologia per tutti gli anni considerati. Sarebbe opportuno approfondire questo aspetto poiché si tratta di una zona in cui trovano collocazione un numero consistente di abitazioni presenti nel dataset: circa il 5\% in ognuno degli stock disponibili. 


\section{Sostenibilità e concorrenzialità per i nuclei in difficoltà abitativa}

\section{Soglie di sostenibilità - Nuclei in difficoltà abitativa}

Dopo aver affrontato, nel precedente capitolo, il tema della sostenibilità e concorrenzialità per i nuclei bolognesi mediani, si passa ad approfondire le possibilità di accesso all'abitazione per famiglie in "difficoltà economica e sociale". Queste famiglie, identificate in questa analisi con quelle che non trovano risposta immediata nell'edilizia residenziale pubblica (ERP) in quanto non sufficientemente ben posizionate nelle graduatorie, versano in condizioni economiche che non garantiscono loro una piena autonomia sul mercato delle locazioni (a canone concordato e, a maggior ragione, a canone libero).

Cambiando la famiglia di riferimento dell'analisi, è solamente il tema della sostenibilità che deve essere riadattato rispetto alla sezione precedente. II tema della concorrenzialità, essendo legato al lato offerta e quindi ai proprietari, non cambia a seconda che l'inquilino sia la famiglia bolognese mediana o quella in difficoltà economica.

Per individuare i limiti di spesa di questa particolare fascia di popolazione, il procedimento è partito dall'ultima graduatoria per l'assegnazione di alloggi ERP. Data la differenza nei dati disponibili tra queste famiglie e quelle mediane, le relative soglie di sostenibilità (definite come $30 \%$ del reddito familiare) saranno calcolate in modo differente. Per il tipo di informazioni disponibili in questo caso abbiamo ritenuto più opportuno calcolare una soglia massima e una minima, partendo dalla distribuzione dei redditi degli individui di riferimento. In questo caso, quindi, non si calcoleranno quattro fasce distinte per il numero di componenti, bensi un valore minimo e massimo all'interno dei quali troveranno collocazione tutte le possibilità di spesa per l'affitto degli individui in "difficoltà abitativa" ${ }^{59}$

Dalla graduatoria ERP, che contiene il reddito ISEE e il numero di componenti del nucleo familiare richiedente, sono stati esclusi i primi 1'500 nuclei, che nei prossimi tre anni dovrebbero veder esaudite le proprie richieste secondo le previsioni di ACER (500 alloggi all'anno per i prossimi 3 anni). Dopo aver ottenuto la distribuzione dei redditi familiari, convertendo il reddito ISEE, si possono calcolare i due valori di riferimento: la soglia minima è identificata come $30 \%$ della media dei redditi del primo quartile della distribuzione; la soglia massima, invece, come $30 \%$ della media dei redditi del quarto quartile della distribuzione stessa.

Poiché non si dispone di tutte le graduatorie stilate nel corso degli anni, otterremo due valori unici, che saranno adottati per tutti gli anni analizzati nella serie storica. Le

\footnotetext{
${ }^{59}$ Per un maggior dettaglio si rimanda alla nota metodologica (sezione "La definizione delle soglie di sostenibilità economica - nuclei in difficoltà abitativa").
} 
soglie così ottenute permettono di sostenere un canone mensile di 68.74€ per il valore minimo e di $495.31 €$ per quello massimo ${ }^{60}$.

La Figura 49 presenta le sei soglie congiuntamente: le quattro rette verdi fanno riferimento ai nuclei mediani, distinti per numero di componenti, mentre le due rette rosse rappresentano il limite inferiore e superiore di sostenibilità per gli individui considerati in "difficoltà abitativa".

Figura 49 - Evoluzione temporale soglie di sostenibilità, nuclei mediani e cittadini in "emergenza abitativa".

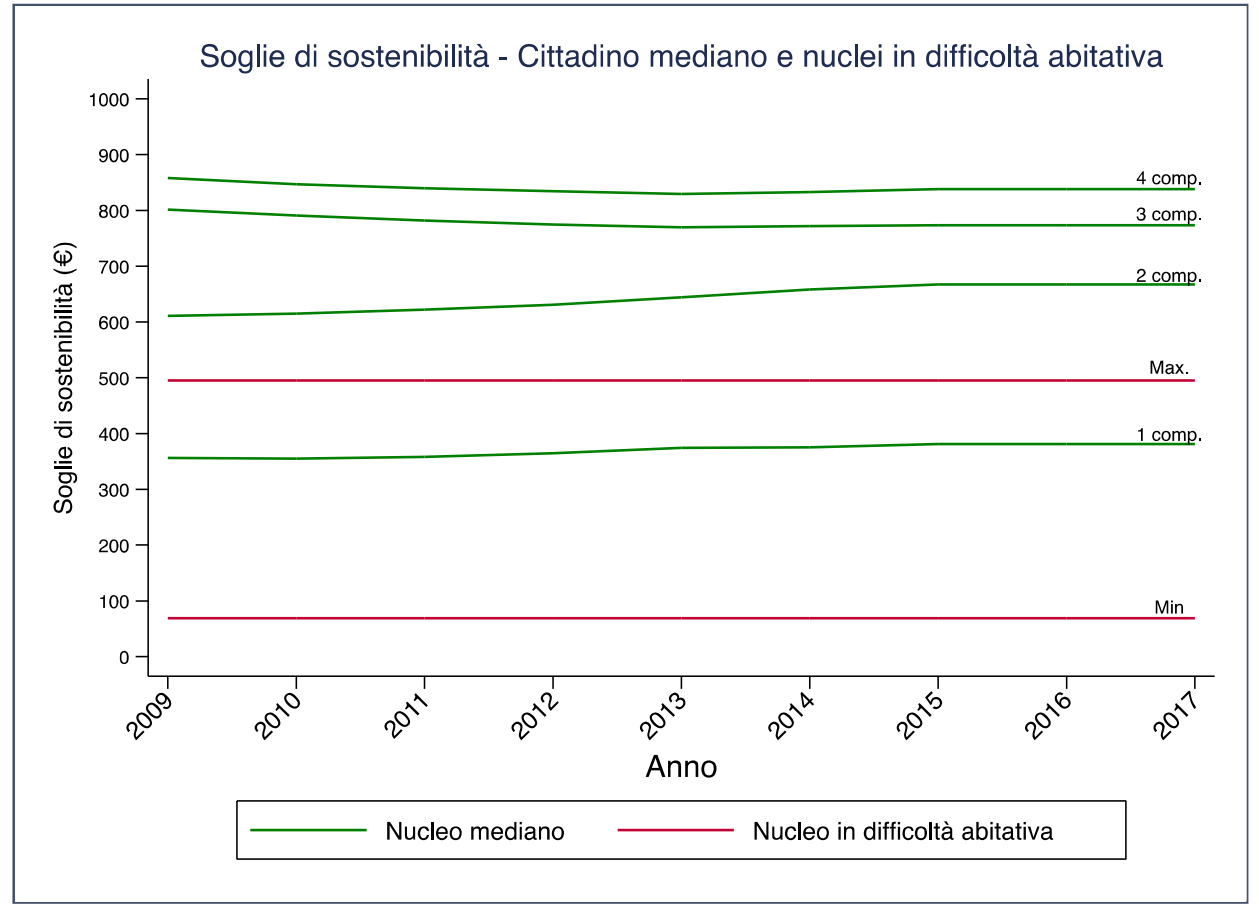

Il valore minimo è molto basso e, già a priori, si può immaginare come gli individui più poveri di questo gruppo non potranno sostenere un canone d'affitto mensile né a canone concordato, né a mercato. L'estremo superiore si colloca al di sopra della soglia di sostenibilità del nucleo familiare unitario mediano, lasciando qualche speranza di sostenibilità per gli individui più ricchi esclusi dalla graduatoria ERP. In realtà, osservando le statistiche descrittive relative al quartile più alto della popolazione di riferimento, nuclei su cui viene calcolata la soglia massima, si tratta di famiglie molto numerose (4 componenti il valore mediano) che difficilmente potranno trovare collocazione nelle abitazioni più piccole (per le quali potrebbe essere maggiore la percentuale di abitazioni sostenibili).

60 I valori corrispondenti dei redditi familiari annuali sono rispettivamente di $2^{\prime} 749.45 €$ e $19 ' 812.63 €$. 
Andando a valutare la percentuale di abitazioni sostenibili per questo particolare gruppo di famiglie, in funzione della soglia minima, nessun alloggio degli stock utilizzati rientra nelle possibilità di spesa mensili per l'affitto delle famiglie più povere escluse dalla graduatoria ERP. Indipendentemente dalla zona e dal numero di vani, tutte le percentuali relative al numero di abitazioni sostenibili risultano nulle.

Diversamente, per gli individui più abbienti di questo particolare gruppo, alcune abitazioni, in particolare quelle più piccole, rientrano nelle possibilità di spesa mensile per l'affitto, come evidenziato nella Tabella 46.

Considerando inizialmente solo gli stock di alloggi 2010 e 2012, indipendentemente dalla zona di applicazione del canone, nessuna abitazione grande (vani superiori o uguali a 5) risulta sostenibile per le famiglie più ricche tra quelle definite in difficoltà abitativa. Gli alti affitti della Zona di Pregio rendono quasi impossibile l'accesso all'abitare di queste famiglie: solamente il $16.67 \%$ e il $70.15 \%$ dei bilocali e monolocali dello stock 2012 risultano essere sostenibili. La composizione numerosa di queste famiglie (nucleo mediano 4 persone) rende impossibile, senza ricadere in una condizione di disagio abitativo, accedere ad alcuni degli alloggi di quest'area. Anche nella Zona A la situazione non risulta essere tanto migliore: tutti i monolocali e i bilocali risultano sostenibili, ma devono essere esclusi per la numerosità dei nuclei di riferimento, e tutte le altre abitazioni, escludendo il 60\% circa dei trilocali, non risultano sostenibili. La Zona B è leggermente più accessibile: tutte le abitazioni con un numero di vani uguale o inferiore a 3 sono sostenibili, unitamente al $65 \%$ circa dei quattro locali. Anche in quest'area nessuna delle abitazioni di dimensioni superiori risulta sostenibile. Se si considera la composizione mediana di queste famiglie e si vuole evitare una situazione di disagio abitativo (numero di persone maggiore rispetto al numero di vani) solamente 1'655 alloggi sulle 9'498 della Zona B (stock 2012), ossia il $17.43 \%$, risultano sostenibili per i più abbienti tra gli esclusi dalla graduatoria ERP. Se si ritiene valida la definizione di disagio abitativo e si considerano tutte le famiglie in questione non diverse dalla composizione mediana (4 persone), solamente 1'655 alloggi su tutti quelli locati a canone concordato nel Comune di Bologna nel 2012 (20'451), ossia l'8.09\%, risultano sostenibili.

Nel 2014 si assiste ad una riduzione generale dei canoni mensili di locazione, sia per contratti concordati, che liberi. Come conseguenza di ciò, effetto molto probabilmente causato dalla riduzione dei valori massimi e minimi del canone concordato definiti nell'accordo 2013, il numero di abitazioni sostenibili aumenta, e questo si verifica anche per le persone più abbienti tra i nuclei in difficoltà economica.

Per lo stock di alloggi 2014, con riferimento alla zona di pregio, le percentuali di monolocali e bilocali sostenibili aumentano, e si registra un valore non nullo (16.69\%) anche per i trilocali. Nella Zona A, oltre al $91.06 \%$ dei trilocali, risulta sostenibile anche il $21.68 \%$ dei quadrilocali, abitazioni effettivamente soddisfacenti in termini di assenza di disagio abitativo per la famiglia mediana di 4 persone. Nella Zona B, infine, anche le abitazioni più grandi risultano in parte sostenibili e non si registrano percentuali negative per nessuna tipologia abitativa. Complessivamente, con riferimento allo 
stock di abitazioni 2014, il numero di abitazioni sostenibili e compatibili con la dimensione mediana della famiglia di riferimento, ossia quelle con un numero di vani pari o superiori a quattro, risultano 4'293 sulle $25^{\prime} 184$ complessivamente locate a canone concordato nel Comune di Bologna. La riduzione dei prezzi di locazione registrata nel 2014 comporta un aumento della percentuale di abitazioni sostenibili e soddisfacenti (dall'8.09\% al 17.05\%) per la famiglia di riferimento del gruppo più abbiente tra le famiglie in difficoltà economica.

Tabella 46 - Percentuale di abitazioni sostenibili a canone concordato rispetto alla soglia massima dei nuclei in difficoltà abitativa, suddivise per zone di applicazione del canone concordato e numero di vani.

\begin{tabular}{|c|c|c|c|c|c|c|c|c|c|c|}
\hline \multirow[b]{3}{*}{ 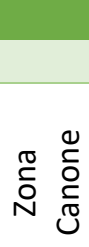 } & & \multicolumn{9}{|c|}{ Abitazioni sostenibili a canone concordato } \\
\hline & & \multicolumn{3}{|c|}{2010} & \multicolumn{3}{|c|}{2012} & \multicolumn{3}{|c|}{2014} \\
\hline & $\frac{\bar{c}}{\bar{m}}$ & 苛 & 芯 & 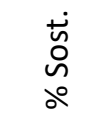 & 苍 & $\begin{array}{l}\dot{\hat{n}} \\
\dot{n}\end{array}$ & $\begin{array}{l}\dot{\tilde{n}} \\
\hat{~} \\
\text { よᄋ }\end{array}$ & 苛 & $\begin{array}{l}\dot{\mathrm{n}} \\
\dot{\sim}\end{array}$ & 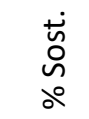 \\
\hline \multirow{6}{*}{ 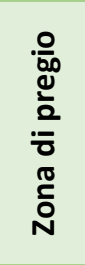 } & 1 & 468 & 326 & 69.66 & 536 & 376 & 70.15 & 628 & 590 & 93.95 \\
\hline & 2 & 287 & 46 & 16.03 & 318 & 53 & 16.67 & 368 & 201 & 54.62 \\
\hline & 3 & 624 & 0 & 0 & 640 & 0 & 0 & 767 & 128 & 16.69 \\
\hline & 4 & 560 & 0 & 0 & 576 & 0 & 0 & 686 & 0 & 0 \\
\hline & 5 & 437 & 0 & 0 & 423 & 0 & 0 & 517 & 0 & 0 \\
\hline & 6 & 635 & 0 & 0 & 626 & 0 & 0 & 767 & 0 & 0 \\
\hline \multirow{6}{*}{ 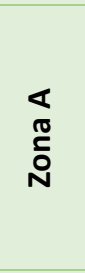 } & 1 & 966 & 966 & 100 & 989 & 989 & 100 & 1165 & 1165 & 100 \\
\hline & 2 & 864 & 864 & 100 & 860 & 860 & 100 & 1074 & 1074 & 100 \\
\hline & 3 & 2491 & 1527 & 61.25 & 2452 & 1511 & 61.62 & 2999 & 2731 & 91.06 \\
\hline & 4 & 1916 & 0 & 0 & 1838 & 0 & 0 & 2265 & 491 & 21.68 \\
\hline & 5 & 963 & 0 & 0 & 929 & 0 & 0 & 1154 & 0 & 0 \\
\hline & 6 & 820 & 0 & 0 & 766 & 0 & 0 & 907 & 0 & 0 \\
\hline \multirow{6}{*}{ 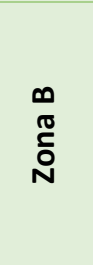 } & 1 & 1203 & 1203 & 100 & 1211 & 1211 & 100 & 1469 & 1469 & 100 \\
\hline & 2 & 1179 & 1179 & 100 & 1188 & 1188 & 100 & 1461 & 1461 & 100 \\
\hline & 3 & 3716 & 3716 & 100 & 3497 & 3497 & 100 & 4406 & 4406 & 100 \\
\hline & 4 & 2632 & 1693 & 64.32 & 2534 & 1655 & 65.31 & 3207 & 3072 & 95.79 \\
\hline & 5 & 799 & 0 & 0 & 764 & 0 & 0 & 983 & 709 & 72.13 \\
\hline & 6 & 310 & 0 & 0 & 304 & 0 & 0 & 361 & 21 & 5.82 \\
\hline
\end{tabular}

\section{Concorrenzialità e sostenibilità per i nuclei in difficoltà abitativa}

Ciò che differisce in questa sezione rispetto all'analisi precedente è la definizione della soglia di sostenibilità; la concorrenzialità, diversamente, non cambia, in quanto riguarda esclusivamente il locatore.

Per individuare i limiti di spesa dei nuclei in difficoltà abitativa, il procedimento ha mosso dall'ultima graduatoria per l'assegnazione di alloggi ERP. Per il tipo di informazioni disponibili in essa, abbiamo ritenuto più opportuno calcolare una soglia 
massima e una minima, partendo dalla distribuzione dei redditi degli individui di riferimento. Poiché non si dispone di tutte le graduatorie stilate nel corso degli anni, otterremo due valori unici, che saranno adottati per tutti gli anni analizzati nella serie storica. Le soglie così ottenute permettono di sostenere un canone mensile di $68.74 €$ per il valore minimo e di $495.31 €$ per quello massimo.

Con una possibilità massima di spesa mensile di 68.74€ risulta evidente che per gli individui più poveri tra quelli esclusi dalla graduatoria di assegnazione dell'edilizia pubblica residenziale, sarà impossibile sostenere qualsiasi affitto nel comune di Bologna.

Diverso è il discorso per la soglia massima: un valore di 495.31€, superiore alla soglia del nucleo bolognese mediano composto da una persona, potrebbe garantire la sostenibilità dell'affitto, almeno in alcune delle zone in cui è stata suddivisa la città. Va però sottolineato come, per i nuclei in difficoltà abitativa, le soglie di sostenibilità non sono differenziate per numerosità familiare e come, in corrispondenza della soglia maggiore, la composizione familiare mediana di questi nuclei è di 4 persone. Come verificato nel capitolo relativo alla sostenibilità di questi nuclei, la maggior parte delle abitazioni sostenibili si registra per gli appartamenti più piccoli, non adatti a queste famiglie.

Figura 50. Distribuzione abitazioni per sostenibilità e concorrenzialità per nuclei in difficoltà abitativa.

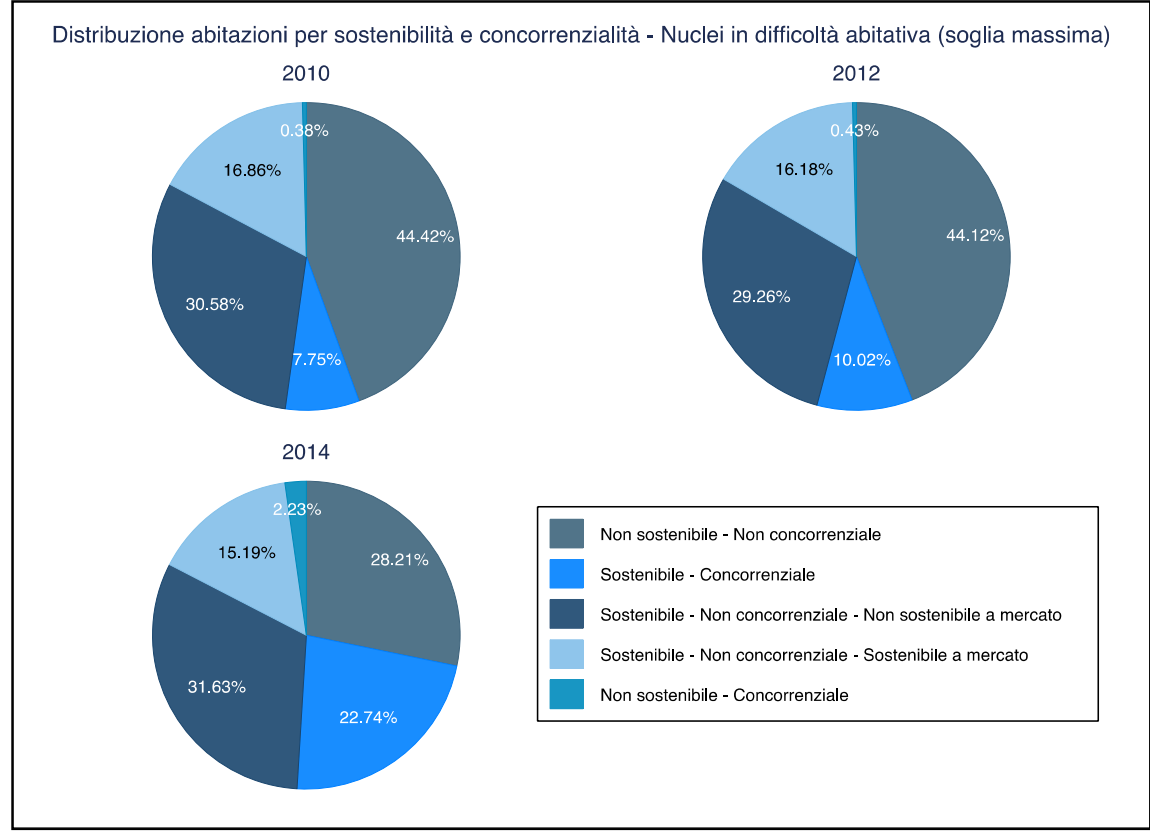

Poiché i dati, per tutti gli anni considerati, confermano che nessun appartamento risulta sostenibile adottando la soglia minima, l'analisi si focalizzerà esclusivamente su quella massima. La Figura 50 rappresenta, per i tre anni di riferimento, la distribuzione degli alloggi degli stock utilizzati tra le diverse combinazioni possibili 
sostenibilità-concorrenzialità, utilizzando come riferimento la soglia massima delle famiglie in difficoltà abitativa. Le abitazioni da guardare con maggior interesse sono quelle sostenibili e concorrenziali, che riescono a soddisfare contemporaneamente le esigenze di locatore e locatario. Secondo le definizioni assunte per concorrenzialità e sostenibilità, si tratta delle abitazioni effettivamente locabili mediante contratti a canone concordato. La percentuale di queste, così come accadeva per il nucleo bolognese mediano, aumenta nel corso degli anni, passando dal $7.75 \%$ iniziale al 22.74\% del 2014. L'aumento consistente di questa percentuale è dettato principalmente da due fattori: da un lato la riduzione dei prezzi di locazione a canone concordato definiti nell'accordo 2013, che rende più sostenibili le abitazioni del campione, dall'altro lo sconto fiscale, a partire dal 2014, per i proprietari, con la riduzione della cedolare secca al $10 \%$, che rende il canone concordato maggiormente concorrenziale rispetto al libero mercato, per cui l'aliquota è del $21 \%$.

Tabella 47 - Percentuali di distribuzione sostenibilità-concorrenzialità per zone di applicazione del canone concordato, applicando la soglia di sostenibilità massima per i nuclei in difficoltà abitativa.

\begin{tabular}{|c|c|c|c|c|c|c|c|}
\hline & & 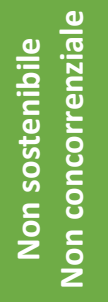 & 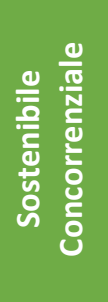 & 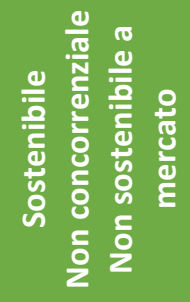 & 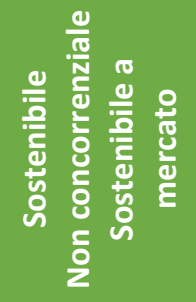 & 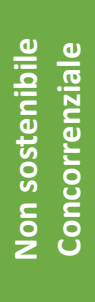 & 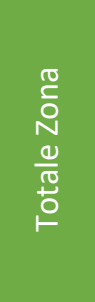 \\
\hline \multirow{3}{*}{ 웅 } & Zona di Pregio & 85.05 & 4.98 & 2.13 & 5.25 & 2.59 & 3011 \\
\hline & Zona A & 58.14 & 6.06 & 24.79 & 11.01 & 0.00 & 8020 \\
\hline & Zona B & 20.79 & 9.98 & 44.02 & 25.19 & 0.02 & 9839 \\
\hline \multirow{3}{*}{$\underset{\sim}{\stackrel{N}{O}}$} & Zona di Pregio & 83.52 & 5.77 & 2.53 & 5.45 & 2.73 & 3119 \\
\hline & Zona A & 57.11 & 6.24 & 24.51 & 12.14 & 0.00 & 7834 \\
\hline & Zona B & 20.48 & 14.53 & 41.95 & 23.03 & 0.02 & 9498 \\
\hline \multirow{3}{*}{$\underset{⿱}{\stackrel{\sim}{~}}$} & Zona di Pregio & 64.83 & 20.87 & 3.75 & 0.00 & 10.55 & 3733 \\
\hline & Zona A & 41.15 & 26.51 & 26.79 & 3.81 & 1.75 & 9564 \\
\hline & Zona B & 6.29 & 20.30 & 44.28 & 29.12 & 0.01 & 11887 \\
\hline
\end{tabular}

Parallelamente all'aumento della tipologia desiderabile, si assiste ad una riduzione delle abitazioni relative alla categoria "non sostenibile e non concorrenziale", che assumeva una quota del $44.42 \%$ nel 2010 per poi scendere a $28.21 \%$ nel 2014 . Le percentuali relative ai restanti gruppi non presentano grandi variazioni per i tre anni di riferimento: le abitazioni "sostenibili e non concorrenziali, sostenibili anche a mercato" si attestano intorno al 30\%; mentre quelle "sostenibili e non concorrenziali, non sostenibili a mercato" registrano sempre valori vicini al $16 \%$. 
Tabella 48. Percentuali di distribuzione sostenibilità-concorrenzialità per zone di applicazione del canone concordato e vani, Bologna, anni 2010-2012-2014.

\begin{tabular}{|c|c|c|c|c|c|c|c|}
\hline Anno & $\begin{array}{l}\text { Zona } \\
\text { Canone }\end{array}$ & Vani & $\begin{array}{c}\text { Non } \\
\text { sostenibile } \\
\text { Non } \\
\text { concorrenziale }\end{array}$ & $\begin{array}{c}\text { Sostenibile } \\
\text { Concorrenziale }\end{array}$ & $\begin{array}{l}\text { Sostenibile } \\
\text { Non } \\
\text { concorrenziale } \\
\text { Non sostenibile } \\
\text { a mercato }\end{array}$ & $\begin{array}{l}\text { Sostenibile } \\
\quad \text { Non } \\
\text { concorrenziale } \\
\text { Sostenibile a } \\
\text { mercato }\end{array}$ & $\begin{array}{c}\text { Non } \\
\text { sostenibile } \\
\text { Concorrenziale }\end{array}$ \\
\hline \multirow{18}{*}{ 옹 } & \multirow{6}{*}{$\begin{array}{l}\frac{0}{00} \\
\frac{0}{20} \\
\frac{0}{0} \\
\frac{1}{0} \\
\frac{N}{2}\end{array}$} & 1 & 15.60 & 30.98 & 4.91 & 33.76 & 14.74 \\
\hline & & 2 & 80.84 & 1.74 & 14.29 & 0 & 3.14 \\
\hline & & 3 & 100 & 0 & 0 & 0 & 0 \\
\hline & & 4 & 100 & 0 & 0 & 0 & 0 \\
\hline & & 5 & 100 & 0 & 0 & 0 & 0 \\
\hline & & 6 & 100 & 0 & 0 & 0 & 0 \\
\hline & \multirow{6}{*}{ đ̊ } & 1 & 0 & 40.99 & 9.32 & 49.69 & 0 \\
\hline & & 2 & 0 & 10.42 & 56.25 & 33.33 & 0 \\
\hline & & 3 & 38.70 & 0 & 56.68 & 4.62 & 0 \\
\hline & & 4 & 100 & 0 & 0 & 0 & 0 \\
\hline & & 5 & 100 & 0 & 0 & 0 & 0 \\
\hline & & 6 & 100 & 0 & 0 & 0 & 0 \\
\hline & \multirow{6}{*}{$\begin{array}{c}\infty \\
\mathbb{2} \\
\check{c}\end{array}$} & 1 & 0 & 71.40 & 1.66 & 26.93 & 0 \\
\hline & & 2 & 0 & 9.84 & 2.37 & 87.79 & 0 \\
\hline & & 3 & 0 & 0.16 & 70.34 & 29.49 & 0 \\
\hline & & 4 & 35.60 & 0.04 & 63.41 & 0.87 & 0.08 \\
\hline & & 5 & 100 & 0 & 0 & 0 & 0 \\
\hline & & 6 & 100 & 0 & 0 & 0 & 0 \\
\hline \multirow{18}{*}{ 궁 } & \multirow{6}{*}{$\begin{array}{l}\frac{0}{80} \\
\frac{0}{2} \\
\frac{2}{0} \\
\frac{\pi}{0} \\
\frac{1}{0}\end{array}$} & 1 & 16.04 & 32.46 & 5.97 & 31.72 & 13.81 \\
\hline & & 2 & 79.87 & 1.89 & 14.78 & 0 & 3.46 \\
\hline & & 3 & 100 & 0 & 0 & 0 & 0 \\
\hline & & 4 & 100 & 0 & 0 & 0 & 0 \\
\hline & & 5 & 100 & 0 & 0 & 0 & 0 \\
\hline & & 6 & 100 & 0 & 0 & 0 & 0 \\
\hline & \multirow{6}{*}{$\begin{array}{l}\varangle \\
\mathbb{\pi} \\
\stackrel{0}{0}\end{array}$} & 1 & 0 & 40.44 & 6.67 & 52.88 & 0 \\
\hline & & 2 & 0 & 10.35 & 55.12 & 34.53 & 0 \\
\hline & & 3 & 38.38 & 0 & 56.28 & 5.34 & 0 \\
\hline & & 4 & 100 & 0 & 0 & 0 & 0 \\
\hline & & 5 & 100 & 0 & 0 & 0 & 0 \\
\hline & & 6 & 100 & 0 & 0 & 0 & 0 \\
\hline & \multirow{6}{*}{ 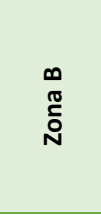 } & 1 & 0 & 71.26 & 1.65 & 27.09 & 0 \\
\hline & & 2 & 0 & 10.61 & 2.36 & 87.04 & 0 \\
\hline & & 3 & 0 & 11.15 & 66.51 & 22.33 & 0 \\
\hline & & 4 & 34.61 & 0.04 & 63.54 & 1.74 & 0.08 \\
\hline & & 5 & 100 & 0 & 0 & 0 & 0 \\
\hline & & 6 & 100 & 0 & 0 & 0 & 0 \\
\hline \multirow{18}{*}{ D্ } & \multirow{6}{*}{$\begin{array}{l}\frac{0}{00} \\
\frac{0}{20} \\
\frac{0}{0} \\
\frac{\pi}{0}\end{array}$} & 1 & 0 & 93.95 & 0 & 0 & 6.05 \\
\hline & & 2 & 7.88 & 41.03 & 13.59 & 0 & 37.50 \\
\hline & & 3 & 54.89 & 4.95 & 11.73 & 0 & 28.42 \\
\hline & & 4 & 100 & 0 & 0 & 0 & 0 \\
\hline & & 5 & 100 & 0 & 0 & 0 & 0 \\
\hline & & 6 & 100 & 0 & 0 & 0 & 0 \\
\hline & \multirow{6}{*}{ 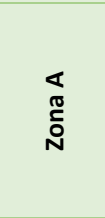 } & 1 & 0 & 91.24 & 1.46 & 7.30 & 0 \\
\hline & & 2 & 0 & 58.01 & 27.47 & 14.53 & 0 \\
\hline & & 3 & 6.64 & 26.81 & 60.15 & 4.10 & 2.30 \\
\hline & & 4 & 74.00 & 1.99 & 19.69 & 0 & 4.33 \\
\hline & & 5 & 100 & 0 & 0 & 0 & 0 \\
\hline & & 6 & 100 & 0 & 0 & 0 & 0 \\
\hline & \multirow{6}{*}{ 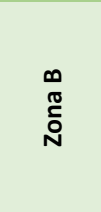 } & 1 & 0 & 96.19 & 1.57 & 2.25 & 0 \\
\hline & & 2 & 0 & 58.25 & 0.75 & 41.00 & 0 \\
\hline & & 3 & 0 & 3.31 & 34.54 & 62.14 & 0 \\
\hline & & 4 & 4.21 & 0.09 & 92.86 & 2.84 & 0 \\
\hline & & 5 & 27.77 & 0 & 72.13 & 0 & 0.10 \\
\hline & & 6 & 94.18 & 0 & 5.82 & 0 & 0 \\
\hline
\end{tabular}


Per approfondire l'analisi, riferita in Figura 50 al Comune di Bologna nel suo complesso, si presenta una suddivisione delle stesse categorie per zone di applicazione del canone concordato. La Tabella 47 presenta le percentuali di ripartizione delle diverse combinazioni sostenibilità-concorrenzialità, per zona di applicazione del canone concordato per gli anni 2010, 2012, 2014.

Anche in questo caso si nota un miglioramento tra il 2010 e il 2014: diminuiscono, indipendentemente dalle zone di applicazione del canone, le quote di abitazioni "non sostenibili e non concorrenziali" e aumentano quelle "sostenibili e concorrenziali". Considerando il gruppo di individui a cui questa sezione dell'analisi fa riferimento, sorprende osservare, nel 2014, percentuali di sostenibilità importanti. La Tabella 48 suddivide le stesse categorie anche in funzione della tipologia di abitazione (numero di vani) e permette di approfondire la situazione relativa alla categoria di interesse (sostenibile e concorrenziale).

Osservando la Tabella 48 è interessante focalizzare l'attenzione sulla colonna relativa alle abitazioni sostenibili e concorrenziali. Per tutti gli anni considerati le percentuali relative alle abitazioni più ampie (cinque e sei locali) risultano nulle. Anche gli appartamenti a quattro vani non si discostano di tanto dal valore nullo, indipendentemente dall'anno e dalla zona di applicazione del canone concordato. Per i trilocali le percentuali cambiano nel tempo. Nel 2010 nessun trilocale, ad eccezione dello $0.14 \%$ degli stessi nella Zona B, risultano essere concorrenziali e sostenibili. Nel 2012 continuano a non esserci trilocali concorrenziali e sostenibili in Zona di Pregio e Zona A, ma aumenta la percentuale nella Zona B (11.15\%). Nel 2014, infine, le percentuali risultano non nulle per tutte le zone: il $4.95 \%, 26.81 \%, 3.31 \%$ del totale dei trilocali di, rispettivamente, Zona di Pregio, A e B.

Complessivamente la percentuale di abitazioni sostenibili e concorrenziali, superiore al $20 \%$ nel 2014, è quasi interamente costituita dalle abitazioni di dimensioni ridotte. Queste abitazioni, però, non sono quelle di interesse per la maggior parte dei nuclei in difficoltà abitativa. La famiglia mediana in questo senso è composta da 4 persone, ed ipotizzando di non voler determinare una situazione di sovraffollamento, la quasi totalità delle abitazioni concorrenziali e sostenibili non è effettivamente adatta a queste famiglie. Considerando anche questo elemento risulta evidente che la disponibilità di tali abitazioni è pressoché nulla per i più abbienti tra coloro che sono esclusi dall'edilizia residenziale pubblica.

La Tabella 49 mostra ancora più efficacemente quanto appena esposto. Se si osserva il numero di vani medio per le abitazioni che soddisfano domanda e offerta di locazione si nota come questa sia molto basso: 1.14 nel 2010, 1.49 nel 2012 e 1.65 nel 2014. È vero che il canone concordato mensile è molto basso per questa tipologia di abitazioni, e questo rende i rapporti di sostenibilità notevolmente inferiori all'unità, ma questa non è condizione sufficiente. La famiglia mediana di questo gruppo di individui, composta da 4 persone, sarà impossibilitata a vivere in abitazioni con un numero di vani medio inferiore a due e una superficie media di $50 \mathrm{mq}$. 
Tabella 49 - Distribuzione alloggi per categorie di sostenibilità e concorrenzialità e relative statistiche descrittive, Comune di Bologna.

\begin{tabular}{|c|c|c|c|c|c|c|c|}
\hline & & 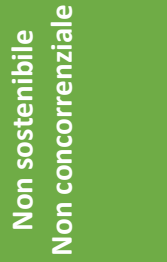 & 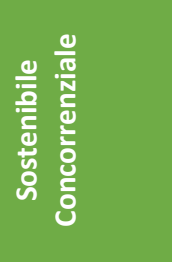 & 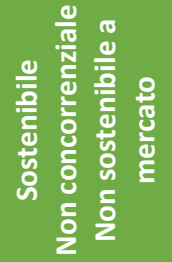 & 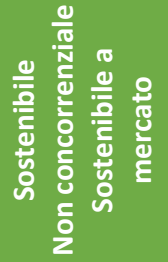 & 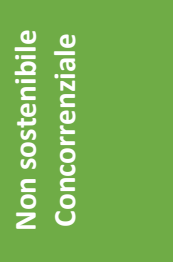 & 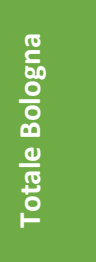 \\
\hline \multirow{6}{*}{ 웅 } & $\begin{array}{l}\text { Numero } \\
\text { abitazioni }\end{array}$ & $\begin{array}{r}9270 \\
(44.42 \%)\end{array}$ & $\begin{array}{r}1618 \\
(7.75 \%)\end{array}$ & $\begin{array}{r}6383 \\
(30.58)\end{array}$ & $\begin{array}{r}3519 \\
(16.86)\end{array}$ & $\begin{array}{r}80 \\
(0.38)\end{array}$ & 20870 \\
\hline & $\begin{array}{l}\text { Dimensione } \\
(\mathrm{mq})\end{array}$ & $\begin{array}{l}102.51 \\
(35.37)\end{array}$ & $\begin{array}{l}43.78 \\
(7.06)\end{array}$ & $\begin{array}{l}71.90 \\
(9.97)\end{array}$ & $\begin{array}{l}54.87 \\
(7.89)\end{array}$ & $\begin{array}{r}49.58 \\
(32.68)\end{array}$ & $\begin{array}{r}80.36 \\
(32.68)\end{array}$ \\
\hline & Numero vani & $\begin{array}{r}4.37 \\
(1.10)\end{array}$ & $\begin{array}{r}1.14 \\
(0.36)\end{array}$ & $\begin{array}{r}3.13 \\
(0.64)\end{array}$ & $\begin{array}{r}2.08 \\
(0.55)\end{array}$ & $\begin{array}{r}1.19 \\
(1.39)\end{array}$ & $\begin{array}{r}3.34 \\
(1.39)\end{array}$ \\
\hline & $\begin{array}{c}\text { Canone } \\
\text { concordato } \\
\text { mensile }(€)\end{array}$ & $\begin{array}{r}626.96 \\
(174.12)\end{array}$ & $\begin{array}{l}363.40 \\
(56.56)\end{array}$ & $\begin{array}{l}444.48 \\
(29.74)\end{array}$ & $\begin{array}{l}383.78 \\
(17.83)\end{array}$ & $\begin{array}{r}523.45 \\
(161.26)\end{array}$ & $\begin{array}{r}509.3 \\
(161.3)\end{array}$ \\
\hline & $\begin{array}{c}\text { Rapporto } \\
\text { canone/soglia }\end{array}$ & $\begin{array}{r}1.02 \\
(0.28)\end{array}$ & $\begin{array}{r}0.59 \\
(0.09)\end{array}$ & $\begin{array}{r}0.72 \\
(0.05)\end{array}$ & $\begin{array}{r}0.62 \\
(0.03)\end{array}$ & $\begin{array}{r}0.85 \\
(0.26)\end{array}$ & $\begin{array}{r}0.83 \\
(0.26)\end{array}$ \\
\hline & $\begin{array}{c}\text { Canone di } \\
\text { mercato } \\
\text { mensile }(€)\end{array}$ & $\begin{array}{l}974.72 \\
(56.79)\end{array}$ & $\begin{array}{l}333.81 \\
(56.79)\end{array}$ & $\begin{array}{l}594.19 \\
(66.18)\end{array}$ & $\begin{array}{l}429.78 \\
(35.23)\end{array}$ & $\begin{array}{r}489.75 \\
(356.55)\end{array}$ & $\begin{array}{r}715.0 \\
(356.6)\end{array}$ \\
\hline \multirow{6}{*}{ 궁 } & $\begin{array}{l}\text { Numero } \\
\text { abitazioni }\end{array}$ & $\begin{array}{r}9024 \\
(44.12)\end{array}$ & $\begin{array}{r}2049 \\
(10.02)\end{array}$ & $\begin{array}{r}5983 \\
(29.26)\end{array}$ & $\begin{array}{r}3308 \\
(16.18)\end{array}$ & $\begin{array}{r}87 \\
(0.43)\end{array}$ & 20451 \\
\hline & $\begin{array}{l}\text { Dimensione } \\
(\mathrm{mq})\end{array}$ & $\begin{array}{l}101.89 \\
(22.81)\end{array}$ & $\begin{array}{r}48.39 \\
(12.29)\end{array}$ & $\begin{array}{r}71.99 \\
(10.08)\end{array}$ & $\begin{array}{l}53.33 \\
(7.47)\end{array}$ & $\begin{array}{r}49.56 \\
(31.88)\end{array}$ & $\begin{array}{r}79.70 \\
(31.88)\end{array}$ \\
\hline & Numero vani & $\begin{array}{r}4.35 \\
(1.11) \\
\end{array}$ & $\begin{array}{r}1.49 \\
(0.80)\end{array}$ & $\begin{array}{r}3.14 \\
(0.65)\end{array}$ & $\begin{array}{r}1.99 \\
(0.55)\end{array}$ & $\begin{array}{r}1.20 \\
(1.40)\end{array}$ & $\begin{array}{r}3.31 \\
(1.40)\end{array}$ \\
\hline & $\begin{array}{c}\text { Canone } \\
\text { concordato } \\
\text { mensile }(€)\end{array}$ & $\begin{array}{r}626.23 \\
(164.67)\end{array}$ & $\begin{array}{l}372.18 \\
(57.24)\end{array}$ & $\begin{array}{l}445.85 \\
(29.46)\end{array}$ & $\begin{array}{l}381.18 \\
(18.37)\end{array}$ & $\begin{array}{r}523.41 \\
(156.68)\end{array}$ & $\begin{array}{r}507.9 \\
(156.7)\end{array}$ \\
\hline & $\begin{array}{c}\text { Rapporto } \\
\text { canone/soglia }\end{array}$ & $\begin{array}{r}0.99 \\
(0.26)\end{array}$ & $\begin{array}{r}0.59 \\
(0.09)\end{array}$ & $\begin{array}{r}0.71 \\
(0.05)\end{array}$ & $\begin{array}{r}0.60 \\
(0.03)\end{array}$ & $\begin{array}{r}0.83 \\
(0.25)\end{array}$ & $\begin{array}{r}0.81 \\
(0.25)\end{array}$ \\
\hline & $\begin{array}{c}\text { Canone di } \\
\text { mercato } \\
\text { mensile }(€)\end{array}$ & $\begin{array}{r}957.85 \\
(365.42)\end{array}$ & $\begin{array}{l}347.05 \\
(64.74)\end{array}$ & $\begin{array}{l}592.45 \\
(65.15)\end{array}$ & $\begin{array}{l}428.73 \\
(31.91)\end{array}$ & $\begin{array}{r}478.95 \\
(344.36)\end{array}$ & $\begin{array}{r}702.2 \\
(344.4)\end{array}$ \\
\hline \multirow{6}{*}{ 守 } & $\begin{array}{c}\text { Numero } \\
\text { abitazioni }\end{array}$ & $\begin{array}{r}7104 \\
(28.21)\end{array}$ & $\begin{array}{r}5727 \\
(22.74)\end{array}$ & $\begin{array}{r}7966 \\
(31.63)\end{array}$ & $\begin{array}{r}3825 \\
(15.19)\end{array}$ & $\begin{array}{r}562 \\
(2.23)\end{array}$ & 25184 \\
\hline & $\begin{array}{l}\text { Dimensione } \\
(\mathrm{mq})\end{array}$ & $\begin{array}{l}111.81 \\
(11.51)\end{array}$ & $\begin{array}{r}50.28 \\
(11.51)\end{array}$ & $\begin{array}{r}80.22 \\
(12.80)\end{array}$ & $\begin{array}{r}64.82 \\
(13.17)\end{array}$ & $\begin{array}{r}71.56 \\
(31.57)\end{array}$ & $\begin{array}{r}79.79 \\
(31.57)\end{array}$ \\
\hline & Numero vani & $\begin{array}{r}4.75 \\
(0.98)\end{array}$ & $\begin{array}{r}1.65 \\
(0.79)\end{array}$ & $\begin{array}{r}3.56 \\
(0.75)\end{array}$ & $\begin{array}{r}2.76 \\
(0.81)\end{array}$ & $\begin{array}{r}2.80 \\
(1.39)\end{array}$ & $\begin{array}{r}3.32 \\
(1.39)\end{array}$ \\
\hline & $\begin{array}{c}\text { Canone } \\
\text { concordato } \\
\text { mensile }(€)\end{array}$ & $\begin{array}{r}629.13 \\
(169.29)\end{array}$ & $\begin{array}{l}365.45 \\
(63.79)\end{array}$ & $\begin{array}{l}436.02 \\
(35.23)\end{array}$ & $\begin{array}{l}364.12 \\
(33.84)\end{array}$ & $\begin{array}{r}534.16 \\
(146.08)\end{array}$ & $\begin{array}{r}465.7 \\
(146.0)\end{array}$ \\
\hline & $\begin{array}{c}\text { Rapporto } \\
\text { canone/soglia }\end{array}$ & $\begin{array}{r}0.96 \\
(0.26)\end{array}$ & $\begin{array}{r}0.56 \\
(0.10)\end{array}$ & $\begin{array}{r}0.66 \\
(0.05)\end{array}$ & $\begin{array}{r}0.55 \\
(0.05)\end{array}$ & $\begin{array}{r}0.81 \\
(0.22)\end{array}$ & $\begin{array}{r}0.7 \\
(0.2)\end{array}$ \\
\hline & $\begin{array}{c}\text { Canone di } \\
\text { mercato } \\
\text { mensile }(€)\end{array}$ & $\begin{array}{r}969.12 \\
(343.42)\end{array}$ & $\begin{array}{l}361.66 \\
(78.48)\end{array}$ & $\begin{array}{l}605.71 \\
(84.40)\end{array}$ & $\begin{array}{l}440.01 \\
(59.04)\end{array}$ & $\begin{array}{r}565.68 \\
(303.17)\end{array}$ & $\begin{array}{r}626.6 \\
(303.2)\end{array}$ \\
\hline
\end{tabular}

Considerando come criterio di assenza di una condizione di disagio abitativo la presenza di un numero di vani almeno pari al numero di persone della famiglia di riferimento, la situazione appare particolarmente critica. Relativamente all'anno 
2014, le abitazioni sostenibili e concorrenziali adatte ad una famiglia di 4 persone in difficoltà abitativa (4 o più locali) sono 0 nella Zona di Pregio, 45 per la Zona A (ossia lo $0.47 \%$ delle abitazioni totali di questa zona) e 3 per la Zona B. Complessivamente queste abitazioni rappresentano lo 0.02\% dello stock complessivo del 2014.

Rendendo la definizione di disagio abitativo meno rigida e considerando accettabili anche i trilocali, gli alloggi concorrenziali e sostenibili adatti a questo gruppo di individui sono 38 per la Zona di Pregio ( $1 \%$ della zona stessa), 849 nella Zona A ( $8.88 \%$ delle abitazioni totali di questa zona) e 149 nella Zona B (1.25\%). A livello complessivo solamente 1'036 (4.11\%) delle abitazioni affittate a canone concordato nel 2014 nel comune di Bologna risultano concorrenziali e sostenibili ed effettivamente alla portata dalle famiglie più abbienti escluse dall'assegnazione di alloggi popolari.

Per riassumere le informazioni riportate nelle tabelle relative alla ripartizione per zona OMI, la Figura 51 rappresenta con diverse gradazioni di colore le percentuali di abitazioni sostenibili e concorrenziali.

\section{Figura 51. Percentuali di abitazioni sostenibili e concorrenziali per zona OMI.}

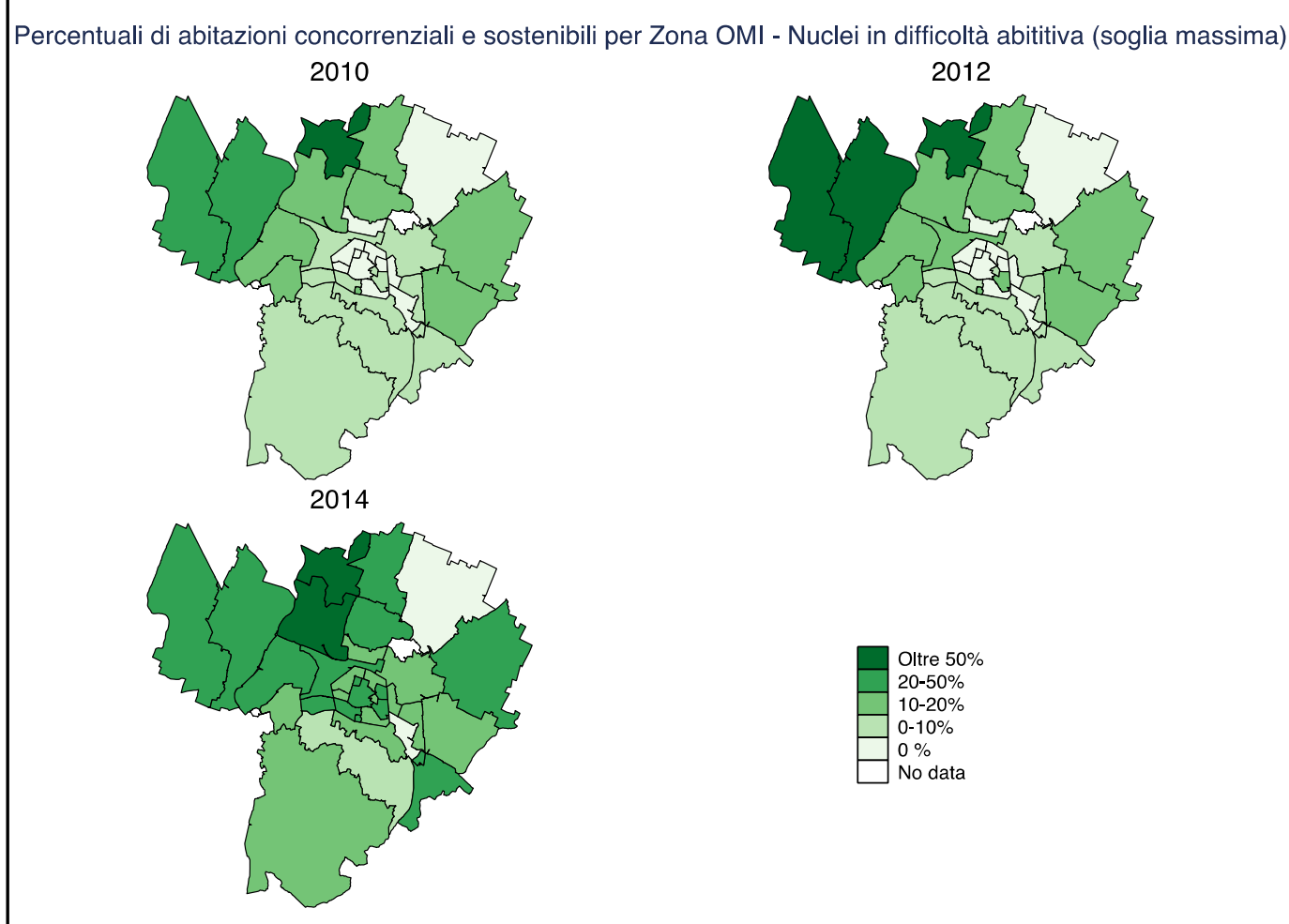

A livello complessivo è possibile osservare un generale aumento di questa tipologia di abitazioni tra il 2010 e il 2014, come testimonia la maggior presenza di tonalità scure nella mappa 2014 rispetto alle prime.

Nel centro storico si passa da una percentuale nulla per quasi tutte le zone nel 2010, a percentuali comprese tra il 10 e il $20 \%$ e tra il 20 e il $50 \%$ nel 2014 . Anche nelle zone 
più periferiche si assiste ad un aumento delle abitazioni che soddisfano i requisiti di domanda e offerta.

In conclusione, però, è importante sottolineare nuovamente come, nonostante le percentuali di abitazioni concorrenziali e sostenibili siano importanti, e non marcatamente inferiori a quelle calcolate per i nuclei bolognesi mediani, queste siano in larga parte costituite dalle piccole abitazioni. Le abitazioni a 4, 5 e 6 locali, e buona parte dei trilocali, non risultano sostenibili in funzione della soglia massima delle famiglie in difficoltà abitativa. Poiché le famiglie escluse dall'edilizia residenziale pubblica sono, secondo le statistiche descrittive calcolate, numerose, l'offerta presente sul mercato del canone concordato appare inadatta a soddisfare le esigenze di queste persone. La situazione è ulteriormente critica poiché, considerando la soglia minima dei nuclei in difficoltà abitativa, ossia quella relativa ai più poveri di questo gruppo, nessuna abitazione presente negli stock analizzati risulta sostenibile. L'offerta del mercato del canone concordato bolognese è inadatta a soddisfare le esigenze dei nuclei in difficoltà e la causa principale va ricercata nella sostenibilità: da un lato $i$ redditi di queste famiglie sono troppo bassi, dall'altro i canoni di locazione sono troppo elevati. 


\section{La domanda di alloggi degli studenti}

\section{Iscritti e fuorisede a Bologna}

Bologna non vanta soltanto l'Università più antica dell'occidente, ma anche una delle più frequentate ed attrattive: stando infatti ai più aggiornati dati pubblicati dal MIUR (riferiti all'anno accademico 2015/2016), soltanto Milano, Roma, Napoli e Torino attraggono più studenti nei rispettivi atenei, pubblici e privati che siano. Non va tuttavia dimenticato che tali città sono di dimensioni ben superiori a quelle di Bologna.

Per valutare gli effetti di una così ampia popolazione studentesca sul mercato abitativo della città occorre disporre di informazioni precise sul numero e sulla provenienza degli studenti che frequentano lezioni e esami presso il campus bolognese. Non sono di interesse ai fini di questa ricerca i partecipanti ai corsi presso sedi distaccate.

Al fine di poter stimare il numero di studenti fuorisede, ossia di coloro che necessariamente devono trasferirsi in città aumentando la domanda di alloggi, in quanto residenti ad una distanza tale da rendere impraticabile l'opzione del pendolarismo, ci si è avvalsi della gentile collaborazione dell'Ateno stesso e dei dati resi disponibili dai loro uffici.

Gli iscritti all'Università di Bologna, presso le facoltà afferenti al campus di Bologna sono 63'615 per l'anno accademico in corso (2017/18): un dato che conferma la tendenza di crescita registrata a partire dal 2014/15, dopo che nei sette anni precedenti si era rapidamente scesi da 66'130 nel 2008/09 a 61547 nel 2013/14 ${ }^{61}$.

Utilizzando i dati forniti dall'Ateneo ${ }^{62}$, riferiti alla provincia di residenza degli iscritti, il numero di fuorisede in cerca di una sistemazione a Bologna è calcolabile nell'ordine delle 36 mila unità circa per l'A.A. in corso. Prima di proseguire, occorre però chiarire la definizione stessa di "fuorisede", e di quanti invece possano frequentare i corsi essendo pendolari, non avendo necessità di trovare un alloggio in città. ${ }^{63}$

L'Agenzia Regionale per il Diritto agli Studi Superiori ER.GO. definisce fuorisede lo "studente che risiede in un Comune la cui distanza dalla sede del corso frequentato sia percorribile, con i mezzi di trasporto pubblico, in un tempo superiore a novanta minuti" 64 .

\footnotetext{
61 Il calo degli studenti è stato ampiamente in linea con una tendenza nazionale dovuta alle difficoltà economiche dvute alla crisi del 2008.

62 Si ringraziano il Prorettore Vicario Degli Esposti e il Dott. Cinti (responsabile Ufficio Data Warehouse e rilevazioni ministeriali).

${ }^{63}$ Questo non esclude la possibilità che tutti gli studenti non fuorisede non cerchino alloggio.

${ }^{64}$ Fonte: www.er-go.it, criteri per la definizione dello studente fuorisede.
} 
L'applicazione di questo criterio ai dati a nostra disposizione, il cui livello di dettaglio giunge fino alla provincia di residenza dell'iscritto (e non al comune), comporta il calcolo dei tempi di percorrenza tra Bologna e i vicini capoluoghi provinciali (si è deciso di non considerare di norma distanze percorribili solo grazie a treni ad alta velocità, il cui costo risulterebbe particolarmente oneroso e potrebbe rendere economicamente più conveniente un affitto in città, nonostante la riduzione della durata dei viaggi). In questo caso, entro il limite dei novanta minuti di viaggio ricadono Ferrara, Forlì, Firenze, Modena, Parma, Prato, Pistoia, Ravenna, Reggio Emilia, Rovigo. Tutti gli studenti provenienti da queste province, oltre ai bolognesi, vengono così esclusi dal novero dei fuorisede. É chiaro, tuttavia, che tale approssimazione non tiene debitamente conto delle difficoltà nel raggiungimento dei comuni più periferici nelle province considerate, spesso meno collegati rispetto ai capoluoghi, anche per conformazione territoriale.

Per questo motivo abbiamo scelto di adottare una definizione più restrittiva che considera pendolari gli studenti residenti nelle province limitrofe a quella di Bologna (Ferrara, Modena, Prato, Pistoia, Ravenna, Firenze) cui si aggiunge Reggio Emilia per l'effettiva rapidità di collegamento ferroviario verso la città felsinea (la scelta tuttavia probabilmente sottostima il numero totale dei fuorisede).

La Tabella 50 affianca al totale degli iscritti, in serie storica dal 2008 al 2018, il conteggio degli studenti fuorisede effettuato secondo i due criteri appena illustrati.

Tabella 50. Totale studenti iscritti ai corsi dell'Università di Bologna nelle facoltà cittadine. Numero e \% di studenti fuorisede secondo due definizioni: criterio delle province limitrofe e criterio ER.GO.

\begin{tabular}{|l|l|l|l|l|l|}
\hline $\begin{array}{l}\text { Anno } \\
\text { accademico }\end{array}$ & $\begin{array}{c}\text { Totale studenti } \\
\text { iscritti }\end{array}$ & $\begin{array}{c}\text { Studenti fuorisede (criteri } \\
\text { ER.GO.) }\end{array}$ & \multicolumn{2}{c|}{$\begin{array}{c}\text { Studenti fuorisede } \\
\text { (escluse province } \\
\text { limitrofe e RE) }\end{array}$} \\
\hline $\mathbf{2 0 0 8 / 0 9}$ & 66130 & 29850 & $45 \%$ & 34144 & $52 \%$ \\
\hline $\mathbf{2 0 0 9 / 1 0}$ & 64230 & 28095 & $44 \%$ & 32265 & $50 \%$ \\
\hline $\mathbf{2 0 1 0 / 1 1}$ & 63340 & 27455 & $43 \%$ & 31563 & $50 \%$ \\
\hline $\mathbf{2 0 1 1 / 1 2}$ & 62847 & 27388 & $44 \%$ & 31474 & $50 \%$ \\
\hline $\mathbf{2 0 1 2 / 1 3}$ & 61778 & 27459 & $44 \%$ & 31446 & $51 \%$ \\
\hline $\mathbf{2 0 1 3 / 1 4}$ & 61547 & 27898 & $45 \%$ & 31905 & $52 \%$ \\
\hline $\mathbf{2 0 1 4 / 1 5}$ & 61716 & 28888 & $47 \%$ & 32894 & $53 \%$ \\
\hline $\mathbf{2 0 1 5 / 1 6}$ & 62740 & 30113 & $48 \%$ & 34142 & $54 \%$ \\
\hline $\mathbf{2 0 1 6 / 1 7}$ & 63382 & 31546 & $50 \%$ & 35582 & $56 \%$ \\
\hline $\mathbf{2 0 1 7} / \mathbf{1 8}$ & 63615 & 32504 & $51 \%$ & 36451 & $57 \%$ \\
\hline
\end{tabular}

Applicando la definizione restrittiva, nell'anno accademico in corso sono oltre 36 mila ( $57 \%$ del totale) gli iscritti che necessitano di un alloggio in città, in linea con una tendenza di crescita, lenta ma costante, a partire dal 2012. Si noti inoltre che l'adozione dei criteri ER.GO., che amplia il numero delle province i cui residenti 
sarebbero considerati pendolari, farebbe calare il numero dei fuorisede nell'ordine del 6-7\%.

La Figura 52 sintetizza i dati riportati finora: la linea continua rappresenta l'andamento del numero degli iscritti alle facoltà bolognesi dall'anno accademico 2008/09 a quello 2017/18; quella tratteggiata il numero di studenti fuorisede secondo la definizione che esclude le province limitrofe e Reggio Emilia.

Figura 52. Andamento del numero totale di studenti iscritti e del numero degli studenti fuorisede (secondo la definizione restrittiva che esclude Bologna, le province limitrofe e Reggio Emilia).

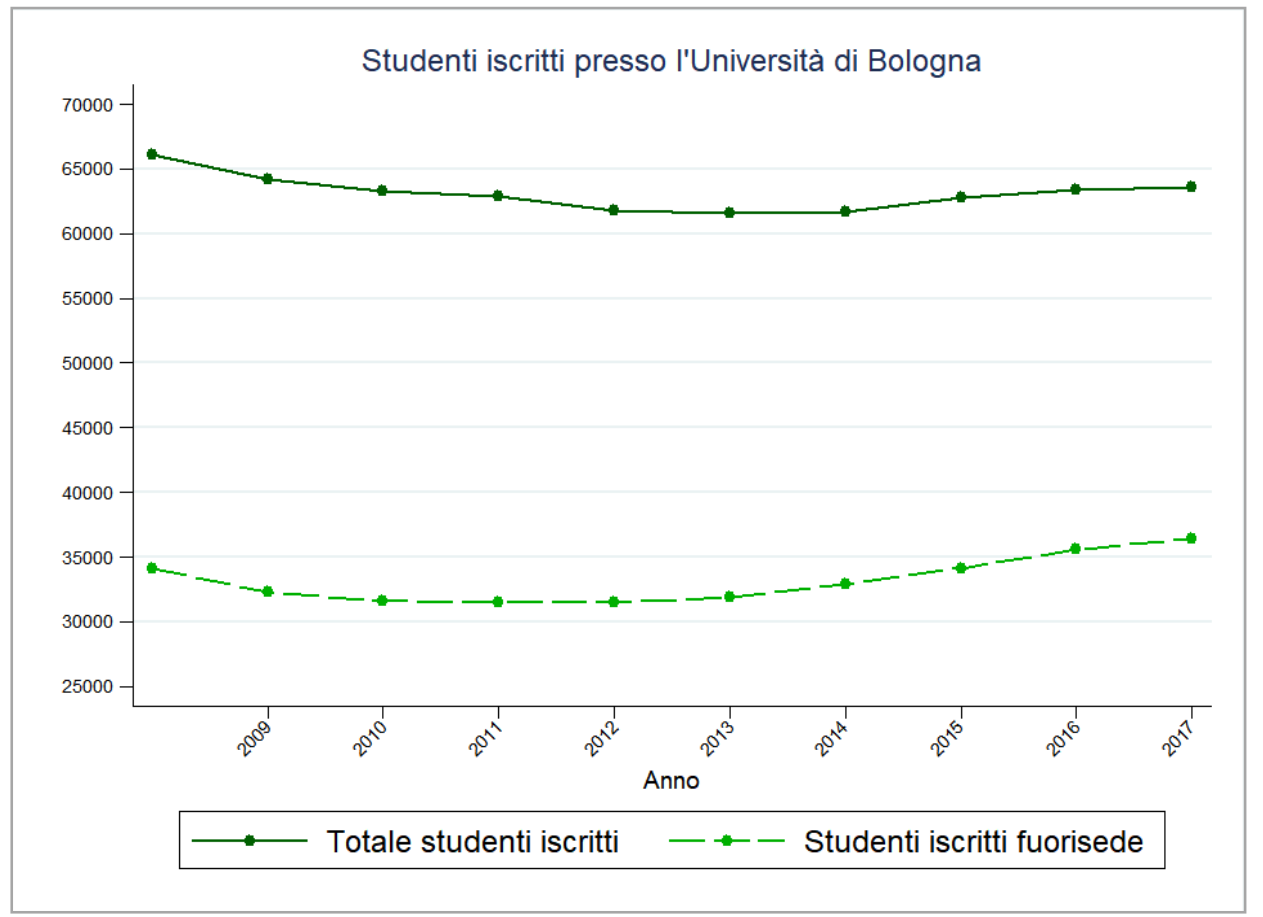

La successiva Figura 53 rappresenta graficamente le province di provenienza degli studenti iscritti presso le facoltà bolognesi, colta all'inizio e al termine del periodo di nostro interesse, ossia negli A.A. 2008/09 e 2017/18. I medesimi dati sono riportati in sintesi nella Tabella 51, accorpando le province secondo la tradizionale suddivisione dell'Italia in macro-aree operata da ISTAT ${ }^{65}$, ma presentando separatamente sia il valore della provincia di Bologna che quello delle restanti province dell'Emilia Romagna.

L'ateneo bolognese si conferma particolarmente attrattivo: nell'anno in corso, gli

\footnotetext{
65 Per la definizione delle macro-aree di residenza è applicata la suddivisione Istat, estrapolando i dati relativi a Bologna e al resto della regione Emilia Romagna. Nord-Ovest: Valle d'Aosta, Piemonte, Liguria, Lombardia. Nord-Est: Veneto, Trentino A.A., Friuli V.G.. Centro: Toscana, Umbria, Marche, Lazio. Sud: Abruzzo, Molise, Campania, Puglia, Basilicata, Calabria. Isole: Sicilia e Sardegna.
} 
iscritti emiliano-romagnoli rappresentano soltanto il $50 \%$ della platea, con un calo evidente della quota di "indigeni" bolognesi (oltre 5'000 unità in meno rispetto ad inizio periodo). A comporre la restante metà della platea sono prevalentemente studenti provenienti dal Centro ( 7 ' 851 , pari al $12 \%$ ed in aumento di un terzo rispetto ad inizio periodo) e dal Sud (16\%), che insieme alle Isole conta più di un quinto degli iscritti.

Figura 53. Provincia di residenza degli studenti iscritti ai corsi dell'Università di Bologna, A.A. 2008/09 e A.A. 2017/18.

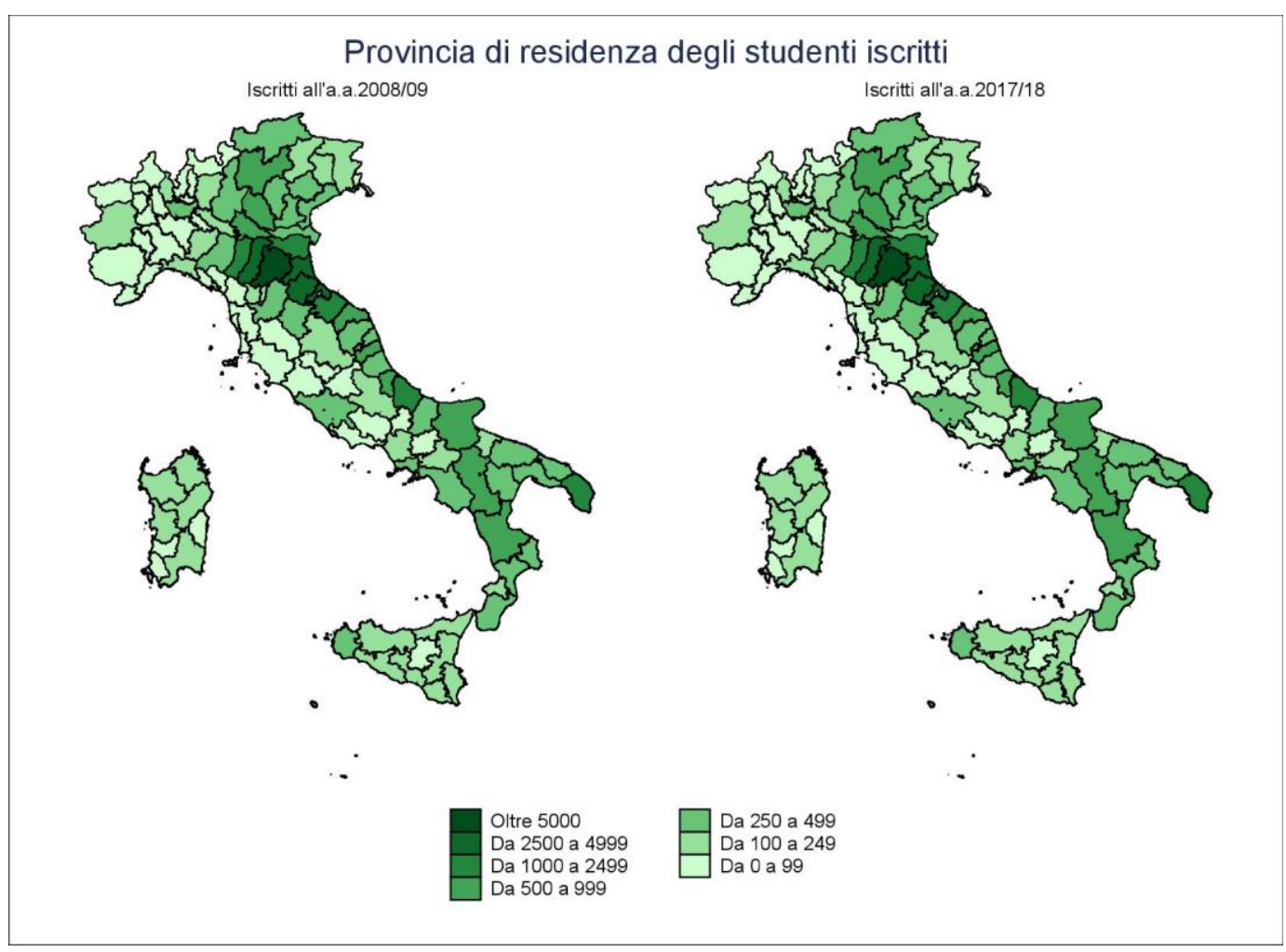

In questo contesto, nelle mappe si evidenziano tonalità più scure lungo la fascia adriatica, mentre, di contro, il Nord-Ovest e l'area compresa tra la Toscana tirrenica e il Lazio, non costituiscono bacini significativi, per la presenza di altri importanti poli universitari come Milano, Torino, e Roma. Infine, occorre rimarcare che gli iscritti residenti all'estero costituiscono solo una parte della quota di studenti internazionali presenti a Bologna: essi infatti risultano normalmente immatricolati ai corsi attivati dall'Ateneo, seguendoli dunque nella loro interezza. Esiste però un numero considerevole di exchange students che arrivano in città per soggiorni brevi (come progetti di scambio Erasmus o Overseas) e che, pur non essendo conteggiati nei totali dei fuorisede stimati in precedenza, vanno comunque ad incrementare la domanda di abitazioni a Bologna. Al difficile percorso di ricerca di un alloggio cui questi ultimi 
vanno incontro è dedicata l'Appendice, al termine del capitolo, che riporta le principali evidenze di un sondaggio in merito, recentemente svolto dall'Università.

Tabella 51. Iscritti ai corsi dell'Università di Bologna per macro-area di residenza e \% sul totale per gli a.a. 2008/09 e a.a. 2017/18. Scarto \% inizio-fine periodo.

\begin{tabular}{|l|r|r|r|r|r|}
\hline Area geografica di residenza & $\begin{array}{c}\text { Iscritti } \\
08 / 09\end{array}$ & $\begin{array}{c}\text { \% su } \\
\text { totale } \\
\text { iscritti }\end{array}$ & $\begin{array}{c}\text { Iscritti } \\
17 / 18\end{array}$ & $\begin{array}{c}\% \text { su } \\
\text { totale } \\
\text { iscritti }\end{array}$ & $\begin{array}{c}\text { Scarto } \\
2008 / 17 \\
(\%)\end{array}$ \\
\hline Bologna & 22488 & 34 & 17138 & 27 & -24 \\
\hline Emilia Romagna & 15448 & 23 & 14582 & 23 & -6 \\
\hline Nord-Ovest & 3026 & 5 & 3212 & 5 & 6 \\
\hline Nord-Est & 4682 & 7 & 4632 & 7 & -1 \\
\hline Centro & 5890 & 9 & 7851 & 12 & 33 \\
\hline Sud & 10368 & 16 & 10397 & 16 & 0 \\
\hline Isole & 2590 & 4 & 3792 & 6 & 46 \\
\hline Estero & 1618 & 2 & 2009 & 3 & 24 \\
\hline ND & 20 & 0 & 2 & 0 & -90 \\
\hline Totale & 66130 & & 63615 & & -4 \\
\hline
\end{tabular}

Alloggiare a Bologna: gli studentati ER.GO.

Oltre al mercato immobiliare, esistono alcune soluzioni appositamente dedicate agli studenti, suggerite dall'Ateneo. Nella pagina web di riferimento ${ }^{66}$, che propone " $i$ servizi, le convenzioni e le risorse utili per trovare casa a Bologna", oltre ad evidenziare le opportunità abitative negli studentati gestiti da ER.GO. - anche per soggiorni di breve durata - sono indicate piattaforme per la ricerca di stanze in affitto e alternative presso strutture private quali i collegi.

Soffermandosi brevemente su questi ultimi, oltre al Collegio Superiore direttamente gestito dall'Alma Mater, sono sei quelli presenti in città cui il portale UniBo rimanda ${ }^{67}$, caratterizzati dalla "dimensione comunitaria, costantemente incentivata grazie alle numerose attività proposte in ambito culturale, accademico e professionale" e dalla presenza di spazi comuni e servizi interni come biblioteche e sale studio (in alcuni casi addirittura palestre).

Dei sei collegi indicati, tre sono riconducibili alla società privata Camplus: "Camplus Alma Mater", "Camplus San Felice", "Camplus Bononia"; gli altri denotano

\footnotetext{
${ }^{66}$ Fonte: www.unibo.it/it/servizi-e-opportunita/borse-di-studio-e-agevolazioni/alloggi-e-sportelloregistrazione-affitti/alloggi-e-residenze-a-bologna/alloggi-e-residenze-a-bologna

${ }^{67}$ Fonte: www.unibo.it/it/servizi-e-opportunita/borse-di-studio-e-agevolazioni/alloggi-e-sportelloregistrazione-affitti/alloggi-e-residenze-a-bologna/bologna-citta-dei-collegi-1
} 
un'ispirazione religiosa, benché la sola "Villa San Giacomo" sia di proprietà ecclesiastica ("Residenza Torleone" e "Collegio di Spagna").

Ai fini di questa ricerca, possiamo però affermare che l'offerta di posti letto all'interno di queste strutture risulti pressoché ininfluente in termini quantitativi e che l'accesso risulti precluso ai più a causa di rette alte, anche al netto delle eventuali detrazioni praticate (si veda a questo proposito la seguente Tabella 52).

Tabella 52. Prospetto posti letto e retta annuale (escluse detrazioni) presso i collegi indicati nel portale UniBo.

\begin{tabular}{|l|l|l|}
\hline $\begin{array}{l}\text { Struttura } \\
\text { Camplus Alma Mater }\end{array}$ & Posti letto & $\begin{array}{l}\text { Retta annuale } \\
\text { (detrazioni escluse) }\end{array}$ \\
\hline Camplus Bononia & 120 posti & $\begin{array}{l}\text { Camera singola: } 12900 \text { euro } \\
\text { Camera doppia: } 10900 \text { euro }\end{array}$ \\
\hline Camplus San Felice & $\begin{array}{l}\text { C8 posti max } \\
(17 \text { appartamenti da 2, 3 o 4 } \\
\text { posti letto) }\end{array}$ & $\begin{array}{l}\text { Camera singola: } 14000 \text { euro } \\
\text { Camera doppia: } 12000 \text { euro }\end{array}$ \\
\hline Residenza Torleone & nd & $\begin{array}{l}\text { Camera singola: } 10200 \text { euro } \\
\text { Camera doppia: } 8200 \text { euro } \\
\text { Bilocale: } 8900 \text { euro }\end{array}$ \\
\hline Villa San Giacomo & nd & 11900 euro \\
\hline Collegio di Spagna & 20 posti & Nd \\
\hline
\end{tabular}

A fronte queste soluzioni esclusive, gli studentati ER.GO. rimangono l'opzione principale. Il servizio offerto da ER.GO. è destinato a studenti fuorisede e prevede l'assegnazione degli alloggi attraverso graduatoria, stilata in base a requisiti di merito (relativi al numero di crediti maturati dagli studenti nell'anno di presentazione della domanda) e di reddito. In particolare, la definizione di quest'ultimo criterio tiene conto di due indicatori: I'ISEE, che non deve essere superiore a 23 mila euro, e l'ISPE, che non deve oltrepassare i 50 mila euro.

Le residenze universitarie ER.GO. riservano inoltre un numero limitato di posti per soggiorni brevi destinato, ad esempio, a ricercatori, studenti di master o exchange students. L'accesso è definito libero in quanto non soggetto a graduatoria, bensì alla semplice disponibilità di stanze. Questa modalità di pernottamento, dunque, pur offrendo un servizio utile e necessario ad alcune categorie di studenti e ricercatori, sottrae una quota minoritaria di alloggi all'utilizzo da parte degli studenti fuorisede normalmente iscritti ai corsi dell'Ateneo.

La Tabella 53 mostra l'andamento negli ultimi dieci anni accademici del numero di posti letto e della retta mensile media. II numero di posti a disposizione sfiora i 1600, con una crescita degli alloggi messi a disposizione degli studenti che si interrompe nel 2013. Le rette mensili rispondono invece alla finalità di offrire una sistemazione a condizioni economiche accessibili: pur crescendo dell' $8 \%$ da inizio periodo le tariffe rimangono molto vicine ai 200 euro. 
Tabella 53. Posti letto e retta mensile media nelle residenze ER.GO., dall'a.a. 2008/09 all'a.a. 2017/18.

\begin{tabular}{|l|r|r|l|l|r|r|}
\hline $\begin{array}{l}\text { Anno } \\
\text { accademico }\end{array}$ & Posti letto & $\begin{array}{l}\text { Retta media } \\
\text { (€) }\end{array}$ & $\begin{array}{l}\text { Anno } \\
\text { accademico }\end{array}$ & Posti letto & $\begin{array}{l}\text { Retta media } \\
\text { (€) }\end{array}$ \\
\hline $\mathbf{2 0 0 8 / 2 0 0 9}$ & 1573 & 190.00 & $\mathbf{2 0 1 3 / 2 0 1 4}$ & 1599 & 200.01 \\
\hline $\mathbf{2 0 0 9 / 2 0 1 0}$ & 1414 & 190.70 & $\mathbf{2 0 1 4 / 2 0 1 5}$ & 1599 & 201.57 \\
\hline $\mathbf{2 0 1 0 / 2 0 1 1}$ & 1414 & 192.27 & $\mathbf{2 0 1 5 / 2 0 1 6}$ & 1599 & 201.57 \\
\hline $\mathbf{2 0 1 1 / 2 0 1 2}$ & 1414 & 194.50 & $\mathbf{2 0 1 6 / 2 0 1 7}$ & 1599 & 202.83 \\
\hline $\mathbf{2 0 1 2 / 2 0 1 3}$ & 1465 & 197.93 & $\mathbf{2 0 1 7 / 2 0 1 8}$ & 1599 & 204.83 \\
\hline
\end{tabular}

Figura 54. Andamento del numero di posti letto totali nelle residenze ER.GO. e della retta mensile media, espressa in euro (secondo asse).

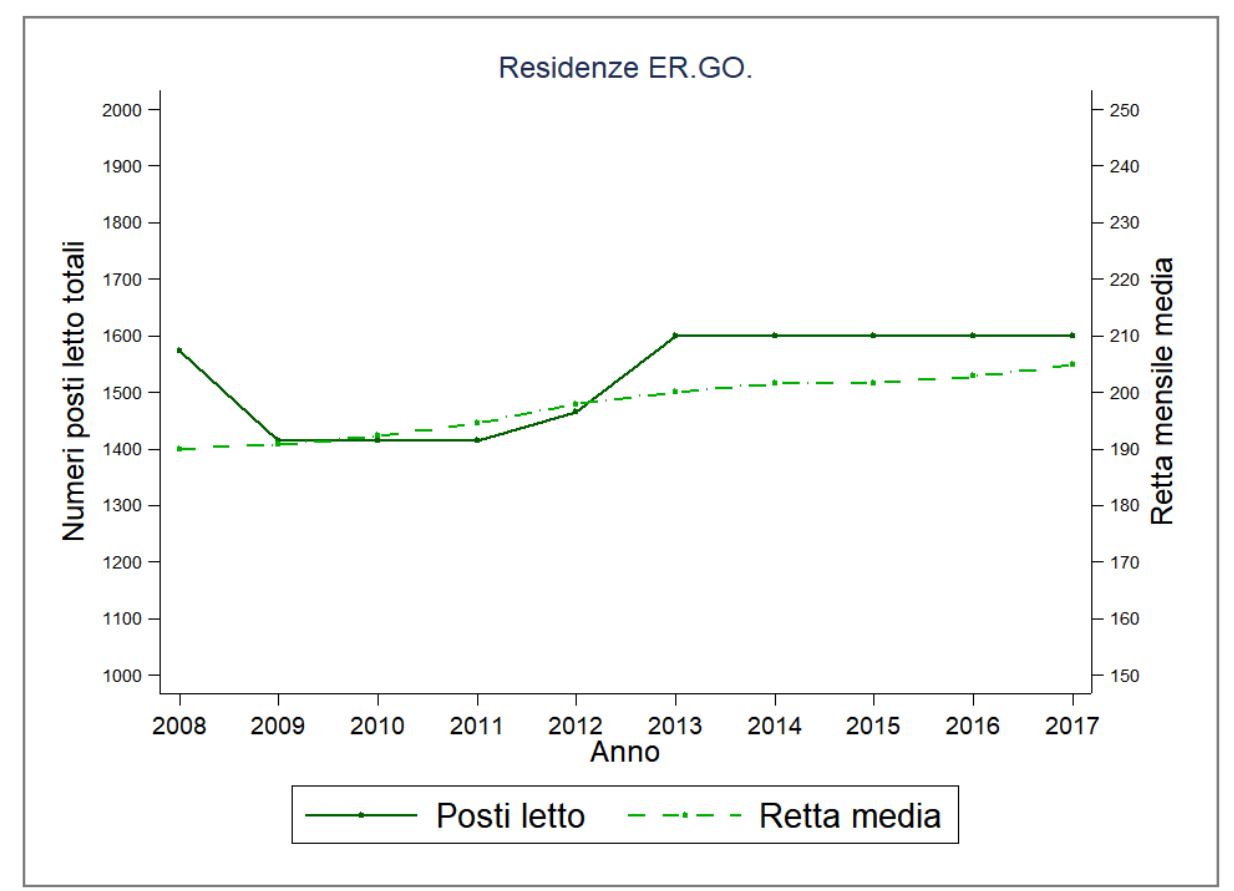

La Figura 55, corredata dalla successiva Tabella 54, rivela la collocazione in città delle residenze universitarie pubbliche, con riferimento alla suddivisione del territorio comunale in zone omogenee OMI. Sei strutture su quindici si trovano all'interno dei viali che delimitano il centro città, caratterizzandosi per dimensioni ridotte (361 posti letto in tutto, poco più di un quinto del totale). Gli studentati più capienti ("Carducci" e "Fioravanti"), oltre alla residenza "Cleto Tomba", si trovano nei pressi del quartiere Bolognina, una delle aree più popolari-e meno ricche della città (zone OMI D7 "Piazza dell'Unità - Matteotti" e D5 “San Donato").

Ancora più distanti dal centro storico e dalle principali sedi dell'Università, sono 415 dei 1'599 posti letto totali, suddivisi tra "Via Larga 1 e 2" e "Marconi" in zona Pilastro (E1) a un estremo, e "Malpighi" e "Galvani" in zona R1 "Agricola Ovest" all'altro. 
Figura 55. Collocazione delle residenze universitarie ER.GO. a Bologna.

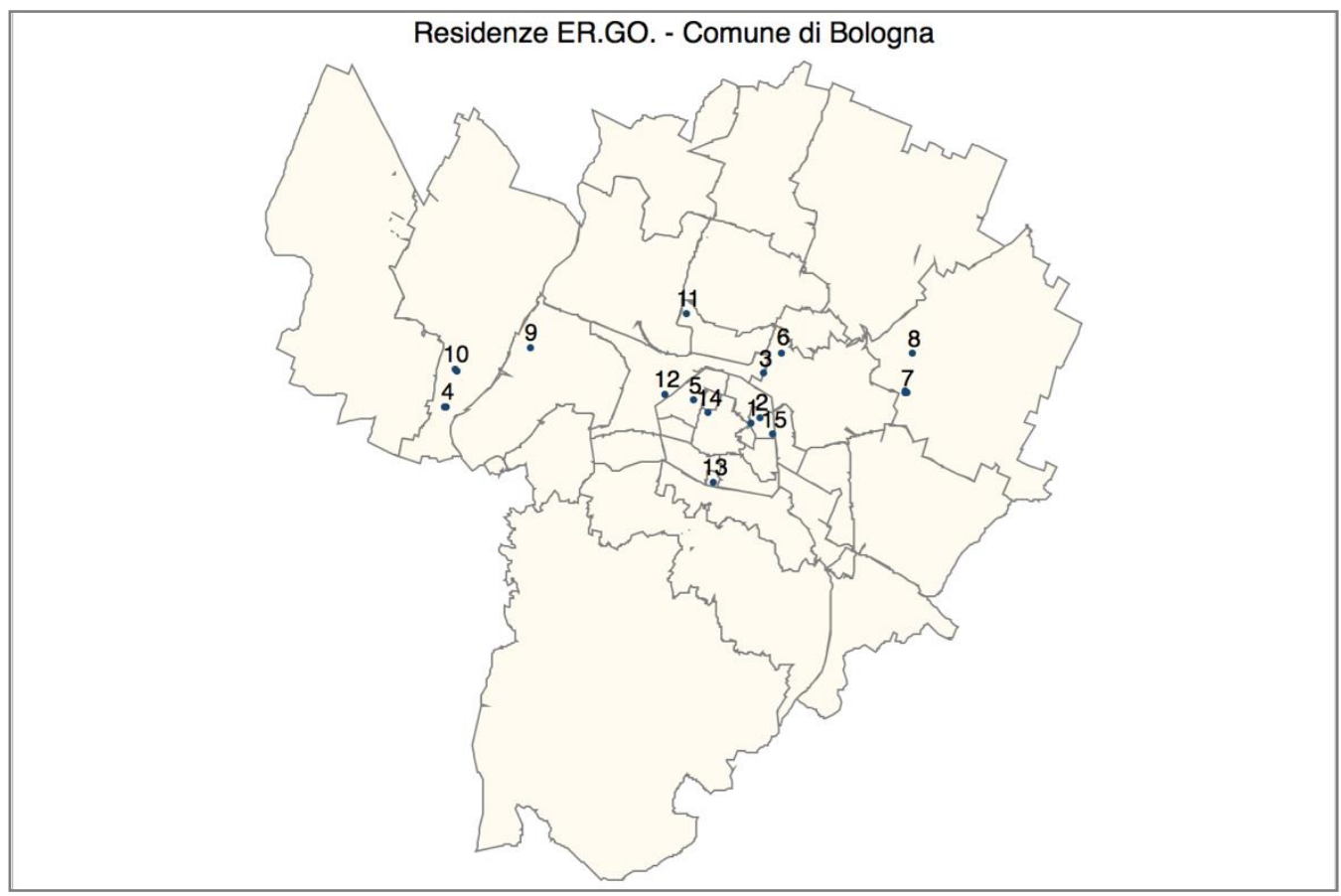

Tabella 54. Posti letto e collocazione delle residenze universitarie ER.GO., in riferimento alla precedente Figura 55.

\begin{tabular}{|l|l|l|l|l|}
\hline$\#$ & Elenco residenze & Posti letto & Indirizzo & Civico \\
\hline $\mathbf{1}$ & MORGAGNI & 59 & Largo Trombetti & $1 / 2$ \\
\hline $\mathbf{2}$ & IRNERIO & 111 & P.zza Puntoni & 14 \\
\hline $\mathbf{3}$ & CARDUCCI & 284 & Via Gandusio & $57-59$ \\
\hline $\mathbf{4}$ & MALPIGHI & 104 & Via Caduti di Casteldebole & 3 \\
\hline $\mathbf{5}$ & CASTELLACCIO & 51 & Via Castellaccio & 18 \\
\hline $\mathbf{6}$ & CLETO TOMBA & 64 & Via Cleto Tomba & $34 / 36 / 38,50$ \\
\hline $\mathbf{7}$ & VIA LARGA 1 e 2 & 116 & Via Del Carpentiere & $15 / 5$ \\
\hline $\mathbf{8}$ & MARCONI & 53 & Via del Pilastro & 164 \\
\hline $\mathbf{9}$ & EX PANIGAL & 64 & Via Emilia Ponente & $50-56$ \\
\hline $\mathbf{1 0}$ & GALVANI & 131 & Via E. De Nicola & 15 \\
\hline $\mathbf{1 1}$ & FIORAVANTI & 240 & Via Greta Garbo & $2 / 2$ \\
\hline $\mathbf{1 2}$ & GHIGI 1 e 2 & 182 & Via Berti & 17 \\
\hline $\mathbf{1 3}$ & MIRAMONTE & 25 & Via Miramonte & $3 / 5$ \\
\hline $\mathbf{1 4}$ & SCHIAVONIA & 31 & Via Schiavonia & 11 \\
\hline $\mathbf{1 5}$ & FORNI & 84 & V.le Filopanti & \\
\hline
\end{tabular}

Ovunque sorgano gli studentati, comunque, occorre far notare la sproporzione tra l'esigenza abitativa complessiva dei fuorisede e ciò che ER.GO. è in grado di offrire: 1'599 alloggi offrono una risposta efficace ma minima a fronte di un numero di fuorisede oltre i 30mila unità (qualunque definizione di fuorisede si intenda applicare). Più precisamente, applicando la definizione precedentemente introdotta, 
nell'anno accademico in corso risultano fuorisede 36 ' 451 iscritti ai corsi; ammettendo che tutti i 1'599 alloggi ER.GO. siano loro destinati, devono accedere al mercato $34^{\prime} 852$ universitari.

\section{Luoghi di interesse e linee di trasporto pubblico notturno}

Oltre a valutazioni di ordine economico, che verranno discusse approfonditamente nel prossimo paragrafo, nella scelta di un alloggio da parte di uno studente influisce in modo particolare la sua vicinanza alle aule universitarie e ai principali luoghi di aggregazione e svago.

Figura 56. Principali punti di interesse UniBo e linee di trasporto pubblico notturno.

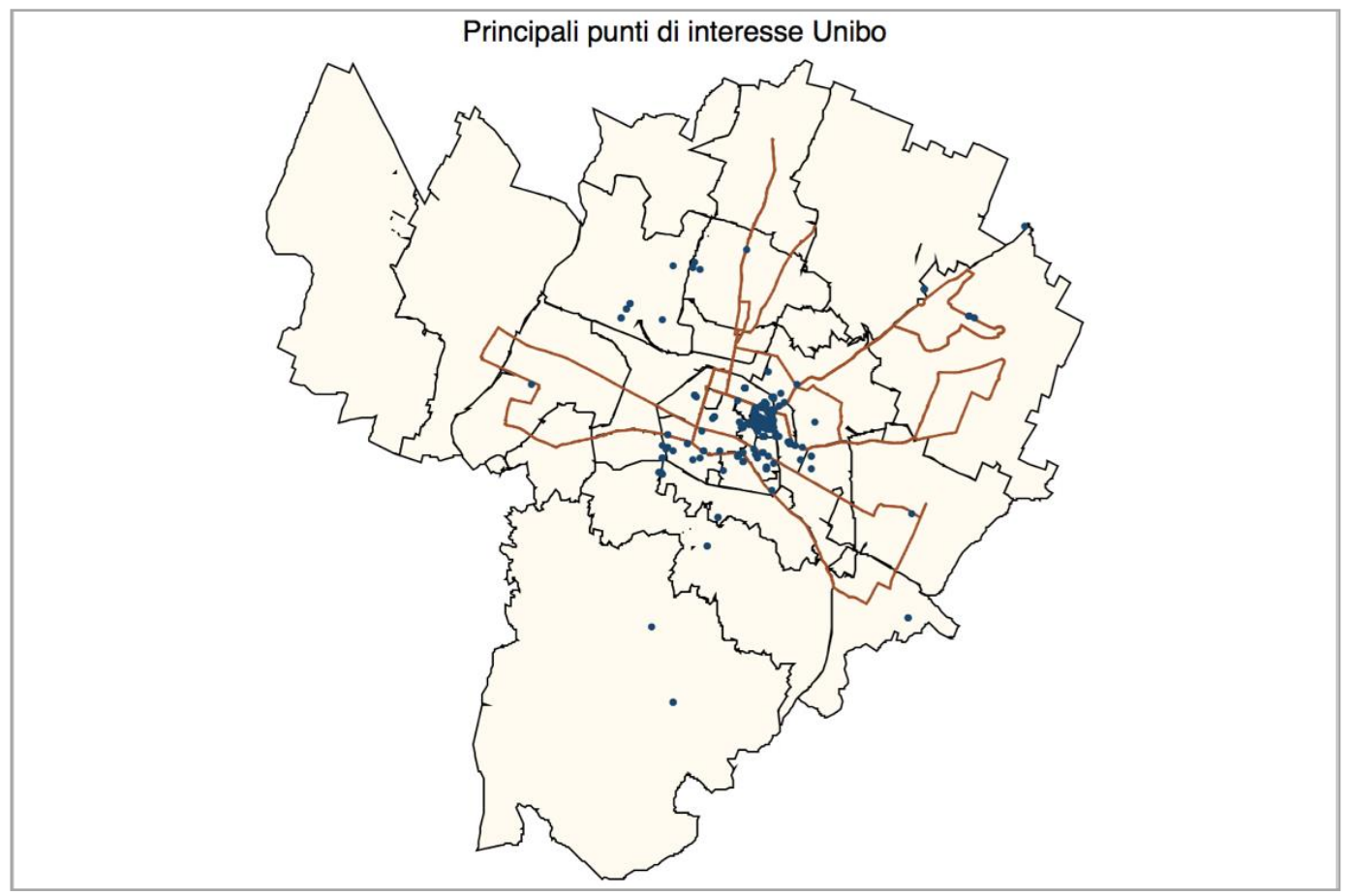

Data la collocazione delle une e degli altri, prevalentemente all'interno del centro storico, l'opzione principale risulta l'affitto dentro la cerchia dei viali o in sua prossimità, in modo da abbreviare gli spostamenti diurni e notturni. Se però raggiungere dipartimenti e facoltà può risultare sostanzialmente facile anche per chi trova una sistemazione più distante, grazie ad una rete di trasporto pubblico capillare, più complessi sono simili spostamenti dalle zone periferiche al centro, se devono avvenire a tarda ora. 
Tabella 55. Linee di trasporto pubblico notturno

\begin{tabular}{|l|l|l|}
\hline Linea & Giorni & Cadenza corse \\
\hline 14N. Via dei Mille - via del Muratore & Venerdì, sabato. & Ogni ora, dalle 0.20 alle 5.20. \\
\hline 20N. Via dei Mille - Pilastro - San Sisto & Venerdì, sabato. & Ogni ora, dalle 0.35 alle 5.35. \\
\hline 25N. Via dei Mille - Dozza & Venerdì, sabato. & Ogni ora, dall'1 alle 4. \\
\hline 61. Via Massarenti - Via Battindarno & Tutti i giorni. & Ogni ora. \\
\hline 62. Corticella - Due Madonne & Tutti i giorni. & Ogni ora. \\
\hline
\end{tabular}

Le linee del trasporto pubblico notturno, rappresentate nella Figura 56 insieme ai principali punti di interesse dell'Università di Bologna, coprono parzialmente il territorio comunale (pur toccando molti dei quartieri più popolosi e i principali assi viari). Ciò avviene grazie ad un intervento finanziato dal Comune di Bologna per oltre 21 mila euro ${ }^{68}$, che da ottobre 2016 ha reso permanenti le linee $14 \mathrm{~N}, 20 \mathrm{~N}$ e $25 \mathrm{~N}$, sperimentate la primavera precedente.

Nonostante l'arricchimento dei collegamenti, tuttavia, questi rimangono inevitabilmente parziali, per ragioni di numero di corse, di serate coperte, di tempi di percorrenza: è lecito dunque immaginare che la domanda di alloggio da parte degli studenti continui a concentrarsi in prossimità del centro storico. Esiste tuttavia una questione, quella della mobilità notturna che si interseca anche con le famiglie lavoratrici, in particolare coloro che lavoro nei servizi essenziali, nella distribuzione, nei servizi alla persona e nello svago che hanno esigenze di mobilità spesso simili agli studenti, anche se per motivazioni opposte.

Questi interessi convergenti nella mobilità diventano però divergenti nella ricerca dell'abitazione. Le aree lungo le linee notturne, per altro quelle fra le più densamente popolate, sono infatti più ambite e tendono a presentare prezzi più elevati, proprio per la domanda studentesca delle stesse.

\section{Gli studenti e il mercato degli affitti a canone concordato}

Gli accordi in materia di locazione concordata a Bologna prevedono una modalità specifica per studenti universitari, definita contratto transitorio, sottoscrivibile con conduttori iscritti "a un corso di laurea o di formazione post laurea [...], in un comune diverso da quello di residenza"69. A fronte di una domanda di alloggio corposa e stabile nel tempo, affittare i propri immobili agli iscritti ai corsi dell'Alma Mater risulta per i proprietari un'opzione vantaggiosa.

\footnotetext{
${ }^{68}$ Fonte: $\underline{h t t p: / / w w w . c o m u n e . b o l o g n a . i t / n e w s / t o r n a n o-i-b u s-n o t t u r n i-t r a-c e n t r o-e-p e r i f e r i e-n e i-~}$ fine-settimana

${ }^{69}$ Fonte: accordo territoriale sui contratti di locazione a canone concordato applicati nel territorio metropolitano di Bologna (accordo 2017 e precedenti).
} 
- Questi contratti hanno una "durata da sei mesi a tre anni, rinnovabili alla prima scadenza"70, dunque consentono al locatore di rientrare in possesso del proprio stabile in tempi decisamente inferiori a quelli previsti da un contratto ordinario (di norma cinque anni secondo la formula del $3+2$ ).

- Date le abitudini e le disponibilità economiche individuali degli studenti, è possibile e frequente che le abitazioni siano affittate a più studenti, che condividono gli spazi comuni o talvolta anche le camere da letto. Ciò comporta che il prezzo di affitto di un alloggio possa essere raggiunto e spesso superato cumulando il contributo di più inquilini, rendendo spesso questa opzione più remunerativa per il proprietario, piuttosto che l'affitto a famiglie.

- I rischi di morosità, essendo distribuiti su più studenti (e sulle relative famiglie), facilmente sostituibili data la domanda, e non sulle sorti di un singolo nucleo rendono gli studenti meno pericolosi.

- A fronte di molteplici risvolti vantaggiosi, questo tipo di contratto è sottoposto alla medesima tassazione (cedolare secca al 10\%) applicata anche ai contratti tradizionali.

- Sfrattare degli studenti morosi è normativamente molto più semplice che sfrattare una famiglia.

Questo quadro, infine, si interseca a volte con condotte illecite, come il subaffitto irregolare o come il pagamento in nero di una quota ulteriore rispetto a quanto dovuto da contratto. I fatti di cronaca riportati dai giornali e i ripetuti interventi negli anni della Guardia di Finanza sull'argomento (alcuni link sono stati riportati in introduzione) ricordano come la soglia di attenzione sull'argomento non possa essere abbassata, sebbene si sia ripetutamente intervenuto.

Detto questo, si ritiene opportuno indagare quali tipologie di abitazione e presso quali zone del territorio bolognese l'affitto a studenti risulti competitivo dal punto di vista del proprietario, sottraendo di fatto parte del patrimonio immobiliare alle esigenze abitative delle famiglie residenti.

Per cominciare, la Tabella 56 riporta il numero di abitazioni affittate a canone concordato, con riferimento all'ultimo stock a nostra disposizione (2014), suddivise per collocazione nelle zone di applicazione del canone e per numero di vani, definiti univocamente ${ }^{71}$.

Rispetto a quanto precedentemente definito analizzando il mercato locativo per i nuclei famigliari, per ciò che concerne gli studenti è più complesso definire il numero di inquilini per abitazione a seconda della quantità di locali. É infatti piuttosto diffuso

\footnotetext{
${ }^{70}$ Fonte: accordo territoriale sui contratti di locazione a canone concordato applicati nel territorio metropolitano di Bologna (accordo 2017 e precedenti).

${ }^{71}$ Si veda la sezione "I vani" nella Nota Metodologica.
} 
l'affitto di camere doppie, che portano dunque due studenti a condividere la medesima stanza, oltre agli ambienti comuni.

Tabella 56. Prospetto del numero di abitazioni locate a canone concordato per zona di applicazione del canone e numero di vani. Stock 2014.

\begin{tabular}{|c|c|c|c|c|c|c|c|}
\hline Vani & 1 & 2 & 3 & 4 & 5 & 6 & Totale \\
\hline Zona di Pregio & 628 & 368 & 767 & 686 & 517 & 767 & 3733 \\
\hline Zona A & 1165 & 1074 & 2999 & 2265 & 1154 & 907 & 9564 \\
\hline Zona B & 1469 & 1461 & 4406 & 3207 & 983 & 361 & 11887 \\
\hline Totale & 3262 & 2903 & 8172 & 6158 & 2654 & 2035 & 25184 \\
\hline
\end{tabular}

Al momento, tuttavia, assumiamo un rapporto di un inquilino per ogni vano, che ci conduce ad un numero di posti letto complessivo, a canone concordato, pari a 83'696, la cui distribuzione per zona e tipologia di abitazione è presentata nella Tabella 57.

Tabella 57. Ipotesi di distribuzione del numero dei posti letto per studenti, a canone concordato, suddivisi per zona di applicazione del canone e numero di vani. Stock 2014.

\begin{tabular}{|l|r|r|r|r|r|r|r|}
\hline Vani & \multicolumn{1}{|c|}{1} & \multicolumn{1}{|c|}{2} & \multicolumn{1}{c|}{3} & \multicolumn{1}{c|}{4} & \multicolumn{1}{c|}{ Totale } \\
\hline Zona di Pregio & 628 & 736 & 2301 & 2744 & 2585 & 4602 & 13596 \\
\hline Zona A & 1165 & 2148 & 8997 & 9060 & 5770 & 5442 & 32582 \\
\hline Zona B & 1469 & 2922 & 13218 & 12828 & 4915 & 2166 & 37518 \\
\hline Totale & 3262 & 5806 & 24516 & 24632 & 13270 & 12210 & 83696 \\
\hline
\end{tabular}

Per poter dimostrare fino a che punto possa essere economicamente vantaggioso affittare a studenti, occorre tuttavia considerare la spesa media che questi ultimi destinano all'alloggio. Per tutte le ragioni elencate finora - permanenza di breve durata, residenza altrove, diffusione di pratiche illecite, incertezza sul numero di inquilini per stanza - non possiamo che affidarci alle stime dell'Università di Bologna ${ }^{72}$, che nella sezione del suo portale web dedicata al costo della vita a Bologna indicano i seguenti valori:

- Camera singola: da 300 a 400 euro più spese;

- Camera doppia: da 200 a 270 euro più spese.

Secondo la nostra assunzione che prevede uno studente per ogni locale dell'abitazione, occorre fare riferimento ai dati relativi alle camere singole, deducendone un costo medio pari a $350 €$. Si tratta indubbiamente di un'altra

72 Fonte: http://www.unibo.it/it/servizi-e-opportunita/studio-e-non-solo/vivere-in-citta/costodella-vita-a-bologna 
inevitabile assunzione, dal momento che tanti fattori possono determinare quella cifra, dalla collocazione più o meno prossima alle sedi universitarie dell'abitazione affittata, al numero di coinquilini, alla sua dotazione di servizi. Anche se questa cifra calasse lievemente, rappresenterebbe comunque una disponibilità che non sempre le famiglie riuscirebbero a garantire.

Tabella 58. Affitto mensile medio e massimo a canone concordato, per numero vani e zona di applicazione del canone concordato. Soglia di sostenibilità degli affitti per famiglie e studenti (monolocali e bilocali: due inquilini; tre e quattro locali: tre inquilini; cinque o più locali: quattro inquilini).

\begin{tabular}{|c|c|c|c|c|c|}
\hline & $\begin{array}{l}\text { Numero } \\
\text { vani }\end{array}$ & $\begin{array}{l}\text { Affitto mensile } \\
\text { medio }\end{array}$ & $\begin{array}{l}\text { Affitto } \\
\text { mensile } \\
\text { massimo }\end{array}$ & $\begin{array}{c}\text { Soglia } \\
\text { sostenibilità } \\
\text { famiglie }\end{array}$ & $\begin{array}{c}\text { Soglia } \\
\text { sostenibilità } \\
\text { studenti }\end{array}$ \\
\hline \multirow{6}{*}{$\begin{array}{l}\frac{0}{50} \\
\frac{1}{2} \\
\frac{0}{0} \\
\frac{0}{0} \\
\frac{\pi}{0} \\
0\end{array}$} & 1 & 416 & 538 & 667 & 700 \\
\hline & 2 & 488 & 633 & 667 & 700 \\
\hline & 3 & 545 & 707 & 773 & 1050 \\
\hline & 4 & 638 & 813 & 773 & 1050 \\
\hline & 5 & 702 & 894 & 838 & 1400 \\
\hline & 6 & 913 & 1165 & 838 & 1400 \\
\hline \multirow{6}{*}{ 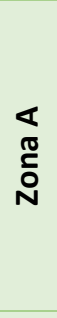 } & 1 & 353 & 460 & 667 & 700 \\
\hline & 2 & 401 & 523 & 667 & 700 \\
\hline & 3 & 446 & 582 & 773 & 1050 \\
\hline & 4 & 526 & 674 & 773 & 1050 \\
\hline & 5 & 576 & 738 & 838 & 1400 \\
\hline & 6 & 704 & 901 & 838 & 1400 \\
\hline \multirow{6}{*}{\begin{tabular}{l}
$\infty$ \\
\multirow{0}{c}{} \\
$\stackrel{0}{N}$
\end{tabular}} & 1 & 298 & 385 & 667 & 700 \\
\hline & 2 & 340 & 441 & 667 & 700 \\
\hline & 3 & 377 & 491 & 773 & 1050 \\
\hline & 4 & 439 & 556 & 773 & 1050 \\
\hline & 5 & 482 & 616 & 838 & 1400 \\
\hline & 6 & 602 & 762 & 838 & 1400 \\
\hline
\end{tabular}

La Tabella 58 esplicita il suddetto fenomeno. In essa sono rappresentati i valori medio e massimo di affitto a canone concordato in riferimento alle abitazioni dello stock 2014. Si ricorda a questo proposito che a definire il prezzo concorrono il numero di vani, la collocazione, la superficie dell'immobile e il numero di elementi di pregio ${ }^{73}$. A fianco sono esplicitate le soglie di sostenibilità per le famiglie bolognesi mediane, ricorrendo in entrambi i casi alla più alta prevista per tipologia di abitazione: due

\footnotetext{
${ }^{73}$ Per una trattazione più dettagliata sulla definizione dei prezzi si rimanda alla Nota Metodologica, nella sezione "Il calcolo dei prezzi a canone concordato".
} 
persone nei monolocali e bilocali, tre persone nei trilocali e quadrilocali, quattro persone nelle tipologie superiori ${ }^{74}$.

Infine, la soglia di sostenibilità per studenti muove dal presupposto che ognuno di loro disponga di una dotazione mensile di 350 euro. Per un confronto efficace, abbiamo previsto i medesimi criteri di riempimento dei locali applicati per le famiglie, pur sapendo che il rapporto di uno studente per locale porterebbe a soglie di sostenibilità più alte in due circostanze (quattro e sei vani) e più bassa per i monolocali.

Alla luce di queste premesse, osserviamo che la soglia di sostenibilità degli studenti è sempre superiore a quella delle famiglie, con uno scarto crescente ad ogni persona aggiuntiva considerata (33 euro di differenza nel confronto tra gruppi o nuclei di due persone, che salgono a 277 per nuclei da tre e 552 per nuclei da quattro).

Se queste differenze non incidono particolarmente in raffronto al canone d'affitto mensile medio (soltanto le abitazioni con sei o più vani in zona di pregio risultano insostenibili in media), esse comunque offrono maggiori garanzie al locatore contro rischi di morosità.

Diverso il discorso se viene considerato l'affitto mensile massimo: in quel caso immobili con quattro o più vani in zona di pregio risultano insostenibili alle famiglie, così come i sei locali in Zona $\mathrm{A}$; di contro tutto rimane sempre sostenibile per gli studenti. Lo scarto tra disponibilità degli studenti e prezzi a canone concordato risulta dunque particolarmente alto e pare consentire margini di guadagno non indifferenti. É vero infatti che esistono parametri massimi definiti dagli accordi che non possono essere contrattualmente superati, ma la definizione dei prezzi in base al numero di elementi di pregio presenti (che devono essere 7 su un elenco di 14 per accedere alla fascia più alta) e la consistente differenza tra tariffe massime e minime all'interno delle stesse fasce di prezzo per pregio e dotazione degli appartamenti, lasciano margini di ampia discrezionalità.

All'estremo, un proprietario potrebbe non ritenere sufficientemente vantaggioso un contratto a canone concordato, nemmeno fosse nella possibilità di applicare il massimo dei prezzi consentiti (potenzialmente superiori agli affitti mensili massimi inclusi in tabella, per costruzione del dato).

In quel caso, escludendo pratiche illegali, occorre sottoscrivere contratti liberi. La normativa a questo proposito non prevede tipologie contrattuali specifiche per studenti, non essendo peraltro i motivi di studio giustificazione accettata per la sottoscrizione di contratti transitori.

Un tradizionale contratto libero non offre però riduzioni IRPEF (in questo caso la tassazione è al $21 \%$ e non al $10 \%$ come per la cedolare secca sul canone concordato) e si presenta più rigido in termini di durata: quattro anni con rinnovo per ulteriori

\footnotetext{
${ }^{74}$ Si veda la sezione "La definizione delle soglie di sostenibilità economica" per il nucleo bolognese mediano, in Nota Metodologica.
} 
quattro, con possibilità di disdire l'accordo al termine del primo periodo o per validi motivi con sei mesi di preavviso.

Peraltro, come evidenziato nella Tabella 59, i contratti a canone di mercato, al netto delle tasse, si rivelano più redditizi soltanto per le abitazioni dai cinque vani in su in Zona di Pregio - ossia quella centrale, più interessante per gli studenti a causa della concentrazione delle strutture accademiche e dei luoghi di svago - e dai quattro vani in su nelle restanti zone.

Tabella 59. Scarto tra canone d'affitto concordato e di mercato al netto delle rispettive aliquote (cedolare secca al $21 \%$ per i contratti di mercato e al $10 \%$ per i contratti a canone concordato). In grassetto le tipologie di abitazione per cui il mercato risulta più redditizio per il locatore.

\begin{tabular}{|c|c|c|c|c|c|c|}
\hline & $\begin{array}{c}\text { Numero } \\
\text { vani }\end{array}$ & $\begin{array}{c}\text { Affitto } \\
\text { mensile } \\
\text { massimo (€) }\end{array}$ & $\begin{array}{c}\text { Affitto } \\
\text { mensile } \\
\text { massimo } \\
\text { netto (€) } \\
\left({ }^{*} 0.79\right)\end{array}$ & $\begin{array}{c}\text { Affitto } \\
\text { mensile } \\
\text { massimo (€) }\end{array}$ & $\begin{array}{c}\text { Affitto } \\
\text { mensile } \\
\text { massimo } \\
\text { netto (€) } \\
\left(*_{0.90)}\right.\end{array}$ & $\begin{array}{c}\text { Scarto } \\
\text { mercato - } \\
\text { canone } \\
\text { Netti (€) }\end{array}$ \\
\hline & & \multicolumn{2}{|c|}{ CANONE DI MERCATO } & \multicolumn{2}{|c|}{ CANONE CONCORDATO } & \\
\hline \multirow{6}{*}{$\begin{array}{l}\frac{0}{00} \\
\frac{0}{0} \\
\frac{0}{0} \\
\frac{0}{0} \\
\frac{0}{0} \\
\frac{0}{N}\end{array}$} & 1 & 438 & 346 & 538 & 485 & -139 \\
\hline & 2 & 578 & 457 & 633 & 569 & -113 \\
\hline & 3 & 741 & 585 & 707 & 636 & -51 \\
\hline & 4 & 915 & 723 & 813 & 732 & -9 \\
\hline & 5 & 1132 & 894 & 894 & 804 & 90 \\
\hline & 6 & 1649 & 1303 & 1165 & 1048 & 254 \\
\hline \multirow{6}{*}{ 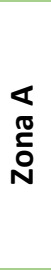 } & 1 & 370 & 292 & 460 & 414 & -121 \\
\hline & 2 & 486 & 384 & 523 & 471 & -87 \\
\hline & 3 & 628 & 496 & 582 & 524 & -28 \\
\hline & 4 & 813 & 642 & 674 & 606 & 36 \\
\hline & 5 & 992 & 784 & 738 & 664 & 119 \\
\hline & 6 & 1399 & 1105 & 901 & 811 & 294 \\
\hline \multirow{6}{*}{ 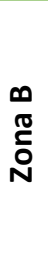 } & 1 & 311 & 246 & 385 & 347 & -101 \\
\hline & 2 & 410 & 324 & 441 & 397 & -73 \\
\hline & 3 & 524 & 414 & 491 & 442 & -28 \\
\hline & 4 & 663 & 524 & 556 & 500 & 24 \\
\hline & 5 & 814 & 643 & 616 & 555 & 89 \\
\hline & 6 & 1098 & 867 & 762 & 686 & 182 \\
\hline
\end{tabular}

A conti fatti, il contratto per studenti a canone concordato si dimostra la tipologia più vantaggiosa, in quanto competitiva economicamente, agevolata dalla cedolare secca al $10 \%$, meno rigida e impegnativa in termini di durata dell'accordo. Ciò nonostante, occorrerebbero indubbiamente un supplemento di indagine per approfondire le dinamiche, i costi, la dislocazione di un mercato che riguarda oltre trentamila studenti ogni anno.

Infine, occorre segnalare un significativo cambiamento nel calcolo delle tasse universitarie, intervenuto a partire da quest'anno. La riforma delle contribuzioni che 
ha definito una soglia ISEE pari a 23 mila euro al di sotto della quale lo studente è totalmente esente, leggiamo dagli organi di stampa ${ }^{75}$, ha comportato l'aumento del $66 \%$ degli aventi diritto all'esonero totale, che passano da 12 mila a 20 mila. Ad essi si aggiungono gli studenti che hanno ottenuto un esonero parziale, che risultano $13 \mathrm{mila}$, quasi il triplo dell'anno precedente.

Questo meccanismo, il cui intento di agevolare le fasce meno agiate della popolazione è indubbiamente apprezzabile, può però dare luogo ad effetti indesiderati. Un iscritto ai corsi potrebbe infatti maturare il diritto all'esonero semplicemente cambiando la propria residenza, distaccandosi così dal nucleo familiare e potendo presentare un ISEE significativamente più basso, pur in assenza di reali condizioni di disagio economico. Va rimarcato, tuttavia, che la residenza non può essere presa a Bologna, per non perdere il diritto a sottoscrivere il contratto a canone concordato riservato agli studenti e altre agevolazioni per fuorisede.

In generale, comunque, questa riforma avrà ricadute anche sul mercato immobiliare, dal momento che una parte più consistente della platea di studenti potrebbe trovarsi a disporre di maggiori risorse da investire per trovare un alloggio di maggiore qualità e in tempi più rapidi. Ecco come una politica di rilevante significato sociale può rischiare di trasformarsi, in certi casi, in un trasferimento di risorse verso i proprietari che locano immobili a studenti.

\footnotetext{
${ }^{75}$ Fonti: "Ateneo di Bologna, a 20mila studenti l'esonero totale dalle tasse" - La Repubblica Bologna (7 febbraio 2018); link: http://bologna.repubblica.it/cronaca/2018/02/07/news/unibo188276090/

"Bologna, l'Alma Mater taglia le tasse agli studenti universitari" - Corriere di Bologna (7 febbraio 2018); link:

http://corrieredibologna.corriere.it/bologna/cronaca/18 febbraio 08/02-bolognadocumentoocorrierebologna-web-bologna-15c45d34-0ca3-11e8-9d55-099ca8cadaf4.shtml
} 
Tra novembre 2017 e gennaio 2018, l'International Desk dell'Università di Bologna ha svolto un sondaggio presso gli studenti stranieri, relativo alle loro condizioni abitative. Sui 7000 questionari distribuiti, il campione di rispondenti ha raggiunto le 1004 unità (di cui il $78 \%$ frequentanti i corsi in città). Presentiamo qui i principali risultati, ricordando che questa ulteriore platea di universitari è soltanto in parte conteggiata nel novero degli iscritti: il 39\% degli intervistati si dichiara infatti exchange student. Questi ultimi costituiscono inevitabilmente un incremento alla domanda di alloggio a Bologna, andando ad aggiungersi al numero dei fuorisede che stimiamo al di sopra dei 36 mila per l'a.a. 2017/18.

Quasi quattro intervistati su dieci dichiarano di aver iniziato a cercare una sistemazione prima ancora di arrivare in Italia, trovandola; per il restante $62 \%$ la ricerca direttamente in città è invece risultata necessaria. Questa fase di ricognizione e selezione delle offerte ha tempi superiori al mese per oltre metà del campione, e compresi tra le due e le quattro settimane per un ulteriore terzo dei rispondenti. Un periodo particolarmente lungo, specie se si considera che frequentemente questi periodi di soggiorno hanno durata inferiore all'anno.

Le difficoltà riscontrate dagli studenti stranieri sono da ricondursi da un lato al costo degli annunci (motivazione addotta dal $26 \%$ dei rispondenti) o ad altre questioni inerenti le condizioni del contratto (cattive condizioni dell'alloggio, richiesta permanenza più lunga), dall'altro a ostacoli di natura culturale. A questo proposito, il $16 \%$ degli intervistati dichiara che il proprietario non accettava exchange students, non conosceva alcuna lingua straniera (11\%), emergevano problemi di comprensione interculturale (6\%); si citano, inoltre, all'interno di una corposa voce "altro" (8\%) anche locatori che non accettano stranieri.

Per trovare una sistemazione, gli studenti provenienti dall'estero hanno fatto ricorso all'aiuto di amici o familiari (24\%), o di servizi di supporto (come gruppi studenteschi, ESN...) - 11\%; mentre per quattro su dieci la ricerca è avvenuta online, tra social network e piattaforme varie (tra cui si cita Airbnb). La ricerca sul posto, tra immobiliari e annunci per strada, vale il $14 \%$, mentre le opzioni previste da Unibo, compresa ER.GO., sono state percorse da poco più di un intervistato su dieci. La tipologia di alloggio modale risulta essere la camera singola in appartamento condiviso (39\%), seguita dalle camere multiple (22\%) e dall'affitto di abitazioni intere (16\%). 
Oltre un terzo degli accordi sottoscritti non risulta regolarmente depositato presso l'Agenzia delle Entrate, a riprova di un malcostume diffuso che con una controparte di questo tipo può prendere ancora più piede.

Quanto agli affitti mensili, benché la categoria mediana ricada nella fascia "tra 250 e 350 " euro, utenze escluse, oltre il $48 \%$ del campione dichiara di pagare più di 350 euro (il $14 \%$ addirittura più di 500 ).

Le abitazioni degli intervistati si collocano prevalentemente fuori dal centro storico, dove alloggia soltanto il $35 \%$ del campione, ma comunque a breve distanza dalle sedi dei corsi: sei su dieci impiegano infatti meno di trenta minuti per raggiungerle, prevalentemente in autobus (42\%) o a piedi (36\%).

In conclusione, il $66 \%$ degli intervistati dà un giudizio positivo alla propria sistemazione. Nonostante questo, lo studio evidenzia alcuni suggerimenti rivolti tanto agli studenti, cui viene consigliato di iniziare la ricerca già prima di arrivare a Bologna, ma anche alle Istituzioni, universitarie e non. A queste ultime in particolare viene raccomandato di porre l'attenzione sulle difficoltà di intermediazione, migliorando $i$ servizi preposti e supportando gli studenti stranieri nelle relazioni con i proprietari, ma anche sull'esigenza di "costruire studentati e ostelli, soprattutto per studenti internazionali che non vengono accettati dai privati".

Si ringrazia il Dott. Danilo Roberto Cinti, responsabile Ufficio Data Warehouse e rilevazioni ministeriali, per la fornitura dei dati illustrati in questa sezione. 


\title{
II mercato turistico
}

\author{
Bologna città turistica
}

Negli ultimi anni si è palesato un nuovo fenomeno che deve essere tenuto in considerazione nell'analisi del mercato locativo cittadino: il turismo. Bologna, infatti, ha visto incrementare negli ultimi anni il flusso turistico in entrata.

Figura 57. Andamento numero passeggeri presso l'aeroporto Marconi di Bologna e numero di presenze turistiche nelle strutture ricettive cittadine.

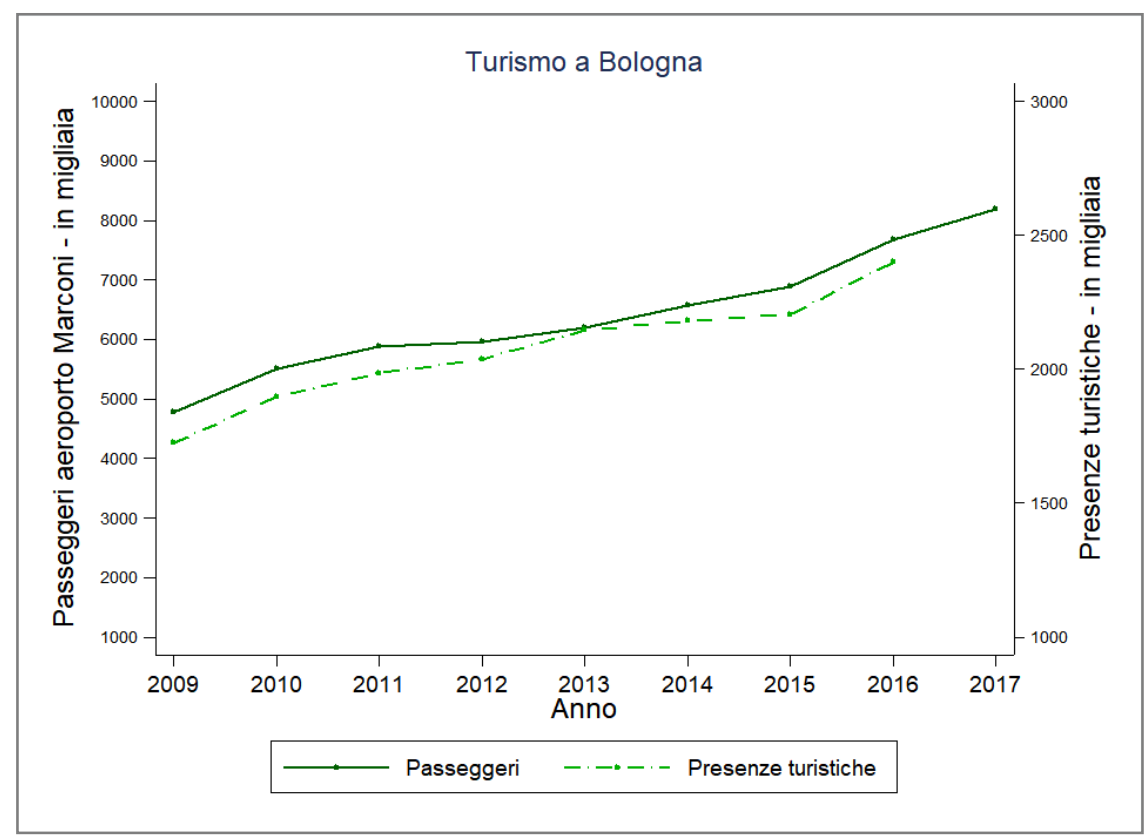

Si tratta sicuramente di un elemento positivo e su cui la città sta investendo molte risorse al fine di stabilizzare il flusso e generare una ulteriore espansione tramite progetti quali il People Mover e FICO. La Figura 57 riporta l'andamento di due indicatori significativi di questa crescita $^{76}$ : i passeggeri che transitano dall'Aeroporto

${ }^{76}$ Fonte dati sul traffico aeroportuale: report annuali Assaeroporti.

http://www.assaeroporti.com/statistiche 201712/

Fonte dati sulle presenze turistiche: rapporti "Il turismo a Bologna e nell'area metropolitana 2015" e precedenti, a cura dell'Area programmazione controlli e statistica del Comune di Bologna; per il 2016, non disponendo di tale rapporto, ci si è basati su fonti giornalistiche, tra cui: "Bologna, boom di turisti: 2,4 milioni nel 2016" - La Repubblica Bologna (19 febbraio 2017). Link:

http://bologna.repubblica.it/cronaca/2017/02/19/news/bologna_boom_di_turisti_2_4_milioni_ nel 2016-158661833/ 
Marconi (quasi raddoppiati tra 2009 e 2017, giungendo a superare gli otto milioni annui), e il numero di presenze turistiche presso le strutture ricettive in città. ${ }^{77}$

A fronte della inelastica (o quantomeno non particolarmente elastica) offerta di alberghi dovuta tendenzialmente alla impossibilità di costruire nell'area comunale nuove strutture (più per vincoli di costo che amministrativi) il fenomeno dell'affitto turistico a breve, conosciuto come Airbnb ${ }^{\text {TM }}$ ovvero dei bed and breakfast e delle case vacanze è andato a ricoprire un ruolo consistente sul mercato cittadino.

Negli ultimi anni, una serie di soluzioni alternative all'albergo propriamente detto hanno mostrato una rapida crescita. Nel momento in cui alcune abitazioni private vengono locate sul mercato degli affitti a breve e brevissimo termine, è evidente che si inneschi un conflitto con il mercato locativo "familiare", ossia quello a lungo termine. Le abitazioni affittate per fini turistici, in assenza di una espansione del numero di alloggi totali, vengono di fatto sottratte dall'offerta di locazione residenziale.

\section{Contesto normativo e dati del Comune di Bologna}

La disciplina delle strutture ricettive dirette all'ospitalità è materia di difficile interpretazione da un punto di vista giuridico, sia per il contesto in continua evoluzione (reale e normativa), sia perché soggetto a potestà legislativa concorrenziale.

A livello nazionale le fonti principali sono il Codice Civile (art. 1571 e seguenti) e il Codice del Turismo (D. Lgs. 79/2011), ma in molti casi il quadro è completato da altre leggi e/o regolamenti: nazionali, regionali e normative comunali. Nel caso dell'Emilia Romagna il testo di riferimento è la legge regionale n. 16 del 28 luglio 2004 ${ }^{78}$, opportunamente modificata e aggiornata da norme più recenti. Essa contiene le definizioni e la disciplina delle diverse strutture ricettive dirette all'ospitalità.

Esistono principalmente cinque diverse tipologie di attività: strutture ricettive alberghiere, strutture ricettive all'aria aperta, strutture extralberghiere, altre tipologie ricettive, agriturismi e fattorie didattiche. Oggetto principale di interesse di questa analisi sono le strutture ricettive extralberghiere e le altre tipologie ricettive, in quanto in queste casistiche rientrano abitazioni di privati affittate a turisti.

La legge regionale 16/2004 distingue le strutture ricettive extralberghiere in: case per ferie, ostelli, rifugi alpini, rifugi escursionistici, affittacamere, case e appartamenti per vacanze. Allo stesso modo le altre strutture ricettive si distinguono in appartamenti ammobiliati per uso turistico e attività saltuaria di alloggio e prima colazione (B\&B).

\footnotetext{
77 Rispetto al quale un incremento è atteso anche per il 2017 e per il quale non disponiamo di dati definitivi, nonostante fonti giornalistiche abbiano riportato, in corso d'anno, una tendenza di crescita intorno al $18 \%$.

78 http://demetra.regione.emilia-romagna.it/al/articolo?urn=er:assemblealegislativa:legge:2004;16
} 
Queste due sotto tipologie non devono essere, secondo la legge, attività organizzate in forma di impresa, a differenza delle sottocategorie appartenenti alla tipologia strutture extralberghiere (affittacamere, case e appartamenti per vacanze) che devono essere gestite in forma imprenditoriale.

Il singolo proprietario, però, anche se persona fisica, potrebbe ricadere nella disciplina delle tipologie extralberghiere, caratterizzate da gestione imprenditoriale, affidandosi ad un'agenzia di gestione immobiliare, come previsto dall'articolo 11 della legge regionale. Il proprietario ha due possibilità per affidarsi all'intermediario: un contratto di mandato o un contratto di locazione ordinario con possibilità di sublocazione. $\mathrm{Nel}$ primo caso l'agente immobiliare percepisce esclusivamente una provvigione e non tutto l'affitto, e la disciplina è quella della sotto categoria case e appartamenti per vacanze (art. 11). Nel secondo caso, invece, l'agente immobiliare assume la gestione dell'immobile pagando un canone d'affitto al proprietario e potendo poi disporre degli interi incassi derivanti dalla sublocazione a turisti. In questo caso, secondo il prospetto del Comune di Bologna ${ }^{79}$, si ricade nella casistica dell'intermediazione da parte di agenzie immobiliari nella locazione di appartamenti ammobiliati a turisti.

Infine, il singolo proprietario persona fisica che vuole locare il suo appartamento mediante portali telematici, potrà farlo ricadendo nella disciplina delle altre tipologie ricettive (appartamenti ammobiliati per uso turistico - art. 12 legge regionale) gestite in forma non imprenditoriale, a condizione che la prenotazione on line non superi i 30 giorni (D.L. 50/2017).

Le abitazioni registrate a settembre 2017 presso il Comune di Bologna, facendo esclusivo riferimento alle strutture ricettive extralberghiere e alle altre tipologie ricettive, risultano essere 382 . Di queste il $53.93 \%$ (206) ricadono nella tipologia di strutture ricettive extralberghiere, mentre il restante $46.07 \%$ (176) sono riconducibili alle altre tipologie ricettive. La Tabella 60 distingue ulteriormente le abitazioni registrate nelle diverse sottocategorie.

Analizzando la proprietà di questi 382 immobili, 108 (28.27\%) sono intestati a persone fisiche, 144 (37.70\%) a imprese individuali e 128 (33.51\%) a società. Sempre in termini di proprietà, 233 (60.99\%) delle 382 registrazioni sono riconducibili a proprietari con una sola registrazione in archivio. Le restanti 149 sono riconducibili a 28 diverse società o imprese individuali. La concentrazione media di questi 149 immobili è di 5 abitazioni per proprietario, ma una singola società è titolare di 33 iscrizioni.

${ }^{79}$ http://informa.comune.bologna.it/iperbole/media/files/tabella ex gdrive 3.pdf

Relativamente a questo prospetto si segnala un'incompatibilità con la classificazione delle attività nel registro comunale. In questa tabella, infatti, l' 'intermediazione da parte di agenzie immobiliari nella locazione di appartamenti ammobiliati a turisti" sembrerebbe ricadere all'interno delle altre tipologie ricettive. Diversamente, nel registro comunale tale sottocategoria è esclusivamente riconducibile alle strutture ricettive extralberghiere, classificazione apparentemente più sensata alla luce della normativa giuridica. 
Escludendo le 34 attività per cui il dato non è riportato, il numero di posti letto totale è di 1 ' 355 , con una media di poco inferiore a 4 posti letto per struttura ricettiva. Questi valori sono in realtà influenzati dai dati mancanti e anche dall'unica "Casa per ferie" registrata che ne offre $42^{80}$.

Tabella 60. Strutture ricettive registrate presso il Comune di Bologna - strutture ricettive extralberghiere e altre tipologie ricettive, settembre 2017.

\begin{tabular}{|c|c|c|c|c|}
\hline \multicolumn{5}{|c|}{ Abitazioni registrate presso il Comune di Bologna (382) - settembre 2017} \\
\hline & & $\mathbf{N}$ & $\begin{array}{c}\% \\
\text { tipologia }\end{array}$ & $\%$ totale \\
\hline \multirow{4}{*}{$\begin{array}{l}\text { Strutture ricettive } \\
\text { extralberghiere } \\
(\mathrm{N}=206 ; 54 \%)\end{array}$} & Case per ferie & 1 & 0.48 & 0.26 \\
\hline & Affittacamere & 37 & 17.96 & 9.69 \\
\hline & Case e appartamenti per vacanze & 162 & 78.64 & 42.41 \\
\hline & $\begin{array}{l}\text { Intermediazione da parte di agenzie } \\
\text { immobiliari nella locazione di } \\
\text { appartamenti ammobiliati a turisti }\end{array}$ & 6 & 2.91 & 1.57 \\
\hline \multirow{2}{*}{$\begin{array}{l}\text { Altre tipologie } \\
\text { ricettive } \\
(\mathrm{N}=176 ; 46 \%)\end{array}$} & $\begin{array}{l}\text { Appartamento ammobiliato per uso } \\
\text { turistico }\end{array}$ & 129 & 73.30 & 33.77 \\
\hline & $\begin{array}{l}\text { Bed \& breakfast - Attività saltuaria } \\
\text { di alloggio e prima colazione. }\end{array}$ & 47 & 26.70 & 12.30 \\
\hline
\end{tabular}

Poiché la disciplina normativa non consente ai "Bed \& Breakfast - attività saltuaria di alloggio e prima colazione" di raggiungere i clienti mediante portali telematici, secondo i dati registrati presso il Comune di Bologna a settembre 2017, 335 abitazioni (il totale delle registrazioni al netto delle 47 che ricadono in questa sottocategoria) sono destinabili ad affitti a breve mediante portali telematici. Si tratta di un numero minimo rispetto al totale delle abitazioni locate a lungo termine che sembrerebbe suggerire come il turismo non rappresenti una minaccia per l'offerta residenziale cittadina. Tuttavia, come verrà descritto nelle prossime pagine, tali numeri sottostimano fortemente la reale entità del fenomeno.

Il fenomeno Airbnb ${ }^{T M}$ a Bologna

Affittare su Airbnb ${ }^{\mathrm{TM}}$

Airbnb $^{\mathrm{TM}}$ è una società nata nel 2007 con la finalità di mettere in contatto persone alla ricerca di alloggi di breve durata con privati disponibili ad affittare singole stanze o la propria abitazione. In dieci anni il portale ha registrato una crescita esponenziale, che I'ha portato a raccogliere oltre quattro milioni di annunci, più delle cinque catene

\footnotetext{
80 Per "Case per ferie" si intendono "strutture attrezzate per il soggiorno a fini turistici di persone singole o gruppi, gestiti[...] senza scopo di lucro da enti pubblici, da associazioni o da enti privati operanti per il conseguimento di finalità sociali [...].
} 
alberghiere più grandi del mondo combinate. II 5 agosto 2017 Airbnb $^{\text {TM }}$, la seconda start-up mondiale per valutazione (31 miliardi di dollari), ha ospitato oltre 2,5 milioni di persone nelle proprie case. L'Italia risulta attualmente il terzo mercato più importante per la società, dopo Stati Uniti e Francia e prima di Spagna e Gran Bretagna ${ }^{81}$.

Negli ultimi anni anche Bologna è risultata coinvolta dalla diffusione capillare del fenomeno: aprire un account e pubblicizzare il proprio annuncio comporta una procedura abbastanza semplice. Più complesso è invece districarsi nelle norme di legge, in una materia che prevede una potestà concorrente tra enti di diverso livello.

Chiunque può diventare "host". "Registrarsi e pubblicare un annuncio è gratuito. Gli annunci disponibili sul nostro sito variano tanto quanto le persone che ospitano, quindi puoi affittare un materasso gonfiabile in un appartamento, case intere, stanze in un bed \& breakfast, case sugli alberi nei boschi, barche galleggianti o castelli incantati"82.

Secondo quanto precisato punto per punto nella pagina del portale "Termini e Politiche", per diventare "host" occorre attenersi a norme e aspettative volte a garantire sia l'affittuario che l'inquilino da episodi di violenza e pericolo, ispirate a principi di rispetto e di non discriminazione, fondate sull'autenticità e sull'affidabilità.

Il portale offre numerosi consigli e buone pratiche di ospitalità, evidenziando gli obblighi di legge previsti nel Paese di residenza. In particolare, per l'Italia ${ }^{83}$ si rimarca I'obbligatorietà della registrazione degli ospiti nel "Portale Alloggiati" delle questure, e la necessità di riscuotere la tassa di soggiorno al momento del check-in. A questo proposito, è da rimarcare l'accordo siglato il 23 ottobre 2017 tra Comune di Bologna e Airbnb ${ }^{\mathrm{TM}}$ per la riscossione automatica di soggiorno: questa procedura, che mette la città felsinea al pari di grandi municipalità come Amsterdam, Parigi, San Francisco, contribuirà a limitare il fenomeno dell'elusione e consentirà un miglior presidio di un settore in grande crescita.

All'esterno della struttura affittata, inoltre, non devono essere affisse insegne o altri elementi distintivi, né possono essere offerti al cliente servizi aggiuntivi (pulizia e cambio della biancheria periodica, somministrazione di pasti).

Riguardo alla registrazione di contratti di affitto, il portale ricorda che deve essere sempre sottoscritto un contratto con l'inquilino ma che questo vada registrato con marca da bollo presso l'Agenzia delle Entrate soltanto se supera la durata di 30 giorni.

In materia di pagamenti, le modalità accettate da Airbnb ${ }^{\text {TM }}$ sono numerose ed escludono il contante; agli ospiti è addebitato l'ammontare dovuto al momento della

${ }^{81}$ Fonte: "Airbnb now has more listings worldwide than the top five hotel brands combined" Bussiness Insider (10/08/2017).

Link: http://www.businessinsider.com/airbnb-total-worldwide-listings-2017-8?IR=T

82 Fonte: https://www.airbnb.it/help/article/18/who-can-host-on-airbnb

${ }^{83}$ Fonte: Airbnb, Ospitare responsabilmente in Italia.

Link: https://www.airbnb.it/help/article/1394/ospitare-responsabilmente-in-italia 
prenotazione e lo stesso è trasferito dal sistema al proprietario dell'annuncio il giorno dopo il check-in, decurtato dei costi di servizio (pari al $3 \%$ più IVA).

Infine, vengono prospettate al locatore due differenti regimi fiscali: I'IRPEF (rispetto al quale i proventi dell'home-sharing si sommano ad altre fonti di reddito) e la Cedolare Secca sugli affitti, al 21\%, non disponibile però per persone giuridiche.

\section{Una ricognizione dell'offerta bolognese su Airbnb ${ }^{\mathrm{TM}}$ : i dati AirDNA.}

Un monitoraggio dettagliato degli annunci su Airbnb ${ }^{\text {TM }}$ risulta alquanto complicato a causa del costante incremento di annunci pubblicati sul sito e della possibilità di disattivare o riattivare un'offerta di ospitalità in qualunque momento. Anche il nome dell'annuncio può essere modificato per risultare più attraente, mentre l'indirizzo preciso dell'immobile viene fornito soltanto a prenotazione effettuata (motivo per cui la georeferenziazione in mappa attraverso coordinate ha un certo livello di imprecisione). Non cambia invece l'identità dell'host, legata all'account sul portale, ma non necessariamente corrisponde al reale proprietario dell'edificio. Ciò comporta che rilevazioni condotte in momenti diversi possano restituire esiti differenti e difficilmente confrontabili tra loro. A fronte di numerosi studi sulla diffusione di Airbnb $^{\text {TM }}$ in città specifiche, quello presentato in queste pagine questo risulta essere uno dei primissimi approfondimenti su Bologna.

In questo lavoro si fa riferimento a due fonti in particolare: i dati di AirDNA ${ }^{84}$, società che monitora oltre 4 milioni di annunci in 40 mila città nel mondo a fini commerciali, e quelli di Tom Slee ${ }^{85}$, conosciuto saggista, blogger, e professionista che si occupa di sharing economy e che ha effettuato periodiche rilevazioni integrali degli annunci in svariate città europee ed americane. ${ }^{86}$

Delle informazioni di AirDNA ci serviremo per offrire una descrizione aggiornata del mercato bolognese, mentre utilizzeremo i database di Tom Slee in quanto consentono di scendere nel dettaglio di ogni singola offerta di alloggio presente al momento della raccolta dati.

La prima importante informazione che mutuiamo dalle rilevazioni AirDNA concerne l'andamento in serie storica del numero degli annunci presenti sul portale Airbnb ${ }^{\text {TM }}$ relativi a Bologna. La Figura 58 sintetizza graficamente la tendenza di crescita degli annunci in corso negli ultimi anni. A gennaio 2018 sono 1'847 gli annunci di appartamenti e "case intere", 1'115 quelli per stanze private e 63 quelli per stanze condivise ( $3^{\prime} 025$ offerte in totale). Soltanto un anno prima, a gennaio 2017 gli annunci

\footnotetext{
${ }^{84}$ Fonte: https://www.airdna.co/

${ }^{85}$ Fonte: http://tomslee.net/airbnb-data-collection-get-the-data

$86 \mathrm{Si}$ rimanda a http://tomslee.net/publications-and-media per un elenco delle pubblicazioni dell'autore, attualmente edite nelle principali lingue del mondo.
} 
erano 2'532, di per sé già in deciso aumento (a inizio 2016 ci si attestava a quota 1'789).

La serie storica mensile consente di raffigurare al meglio gli effetti prodotti dalla possibilità di disattivare momentaneamente i propri annunci, che conducono a saltuarie inversioni di tendenza. La Figura 59 riguarda invece l'andamento delle entrate mensili del mercato Airbnb ${ }^{\mathrm{TM}}$ a Bologna ed offre una rappresentazione evidente della crescita del mercato stesso e della sua stagionalità (per quanto, fa notare AirDNA, molto meno pronunciata di quella registrata in località di turismo estivo o invernale).

Figura 58. Numero di annunci presenti su Airbnb ${ }^{\text {TM }}$ per la città di Bologna. In blu le "case intere/appartamenti", in azzurro le "stanze private", in verde le "stanze condivise". Serie storica su base mensile, a partire da agosto 2015. Fonte grafico: AirDNA.co

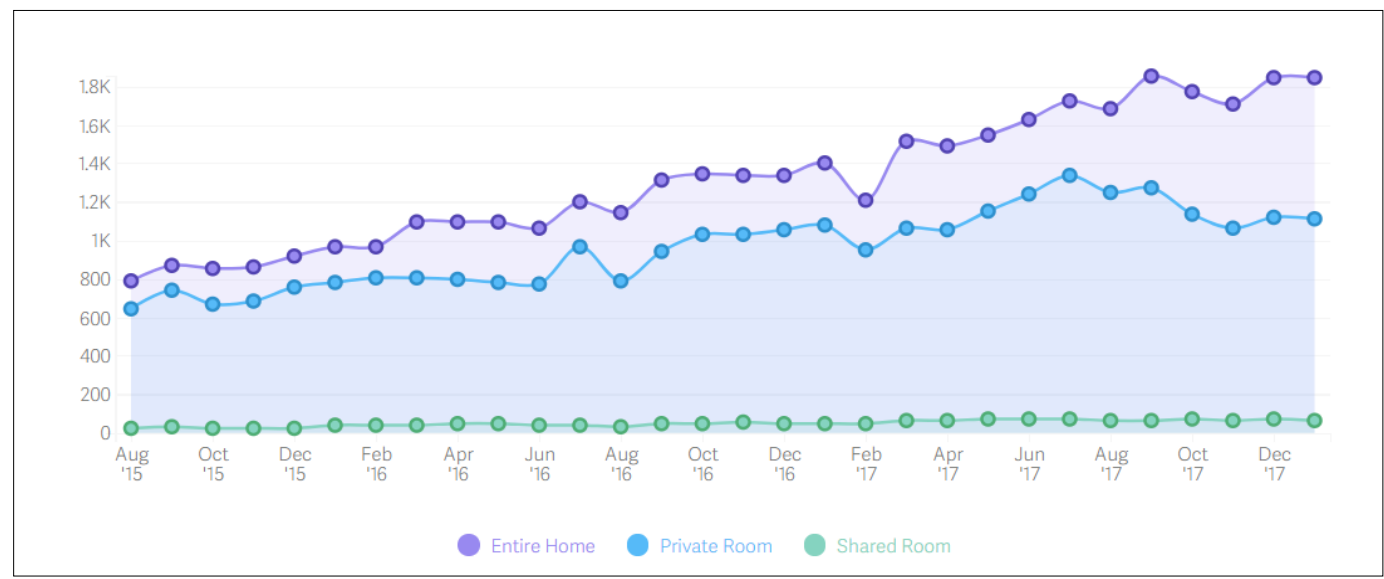

Da inizio 2016 in poi possiamo assistere a due momenti di picco che si susseguono annualmente, ossia la primavera (da marzo a maggio) e, in maniera più marcata, i mesi di settembre e ottobre. Per quanto riguarda il picco del mese di settembre, in particolare, è possibile ipotizzare che sia da imputarsi anche all'arrivo in città, alla vigilia della ripresa dei corsi, di oltre trentamila studenti universitari fuorisede, parte dei quali potrebbero necessitare di alloggi temporanei in attesa di trovare una sistemazione definitiva in città.

Al netto del ciclo stagionale, è lampante l'incremento del giro d'affari di Airbnb ${ }^{\text {TM }}$ nella città felsinea: nel 2017 le entrate totali hanno sfiorato i 27milioni, con un aumento del $66 \%$ rispetto all'anno precedente. Nel solo mese di settembre 2017 le entrate da annunci Airbnb ${ }^{\mathrm{TM}}$ a Bologna hanno superato i 3 milioni e mezzo di euro. Gli incassi sono dati prevalentemente dagli affitti di "case intere", più numerose in termini di annunci e naturalmente più costose. 
Figura 59. Serie storica delle entrate mensili generate dagli affitti di "case intere/appartamenti" (in blu), "stanze private" (in azzurro), "stanze condivise" (in verde). Valori espressi in milioni di euro. Fonte grafico: AirDNA.co

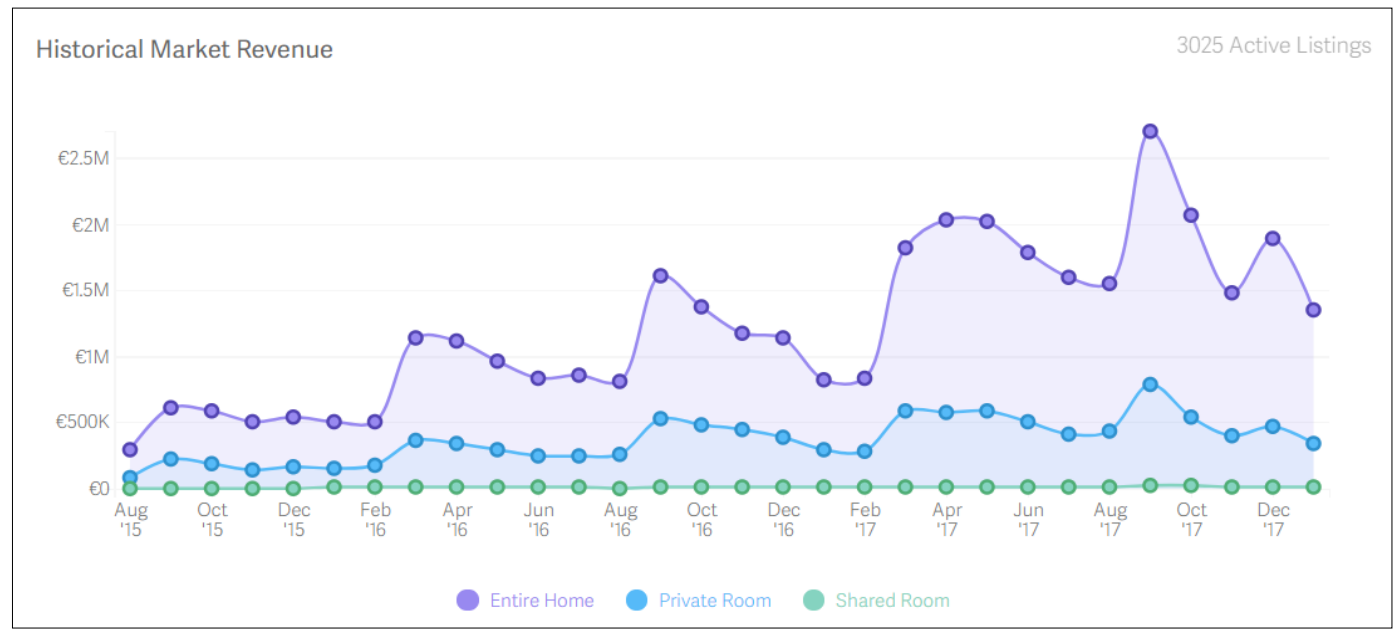

La Tabella 61, infine, presenta l'andamento mensile di prezzi e tasso di locazione mediano per "case intere" e stanze private. Osservando il tasso di locazione mediano, calcolato dividendo il numero di pernottamenti nel mese considerato sul totale dei posti disponibili per la relativa categoria di annuncio, notiamo una sostanziale discrepanza tra "case intere" e stanze private; ciò si annulla nei mesi di settembre e ottobre - periodo di punta per il mercato di Airbnb ${ }^{\mathrm{TM}}$ a Bologna. Un dato che potrebbe rafforzare l'ipotesi relativa all'afflusso di studenti fuorisede in attesa di trovare una sistemazione stabile entro l'inizio dei corsi: le stanze private sono d'altronde meno costose e la condivisione degli spazi abitativi è costume diffuso all'interno di quella specifica categoria di inquilini.

Tabella 61. Andamento mensile per l'anno 2017 di prezzo medio e tasso di locazione mediano per

"Case intere/appartamenti" e "Stanze private". Il tasso di locazione mediano è calcolato dividendo il numero di pernottamenti nel mese sul totale dei posti disponibili (escludendo gli annunci che non hanno mai registrato prenotazioni)

\begin{tabular}{|c|c|c|c|c|c|c|c|c|c|c|c|c|c|}
\hline \multicolumn{2}{|c|}{2017} & Gen & Feb & Mar & Apr & Mag & Giu & Lug & Ago & Set & Ott & Nov & Dic \\
\hline \multirow{2}{*}{ 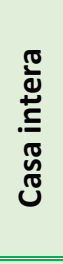 } & $\begin{array}{l}\text { Prezzo } \\
\text { Medio }(€)\end{array}$ & 71 & 67 & 84 & 79 & 79 & 75 & 73 & 70 & 86 & 76 & 73 & 80 \\
\hline & $\begin{array}{l}\text { Tasso di } \\
\text { locazione } \\
\text { mediano } \\
(\%)\end{array}$ & 38 & 58 & 55 & 73 & 69 & 65 & 58 & 63 & 73 & 74 & 60 & 50 \\
\hline \multirow{2}{*}{ 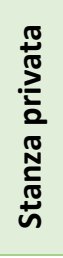 } & $\begin{array}{l}\text { Prezzo } \\
\text { Medio }(€)\end{array}$ & 37 & 34 & 45 & 40 & 40 & 36 & 35 & 33 & 41 & 38 & 37 & 40 \\
\hline & $\begin{array}{l}\text { Tasso di } \\
\text { locazione } \\
\text { mediano } \\
(\%)\end{array}$ & 31 & 46 & 43 & 53 & 54 & 53 & 42 & 61 & 71 & 74 & 57 & 48 \\
\hline
\end{tabular}


Dopo aver verificato attraverso i dati AirDNA l'incremento esponenziale del mercato degli affitti brevi su Airbnb ${ }^{\text {TM }}$, ricorriamo ai database di Tom Slee, saggista nell'ambito della "sharing economy", per studiare l'offerta bolognese abitazione per abitazione.

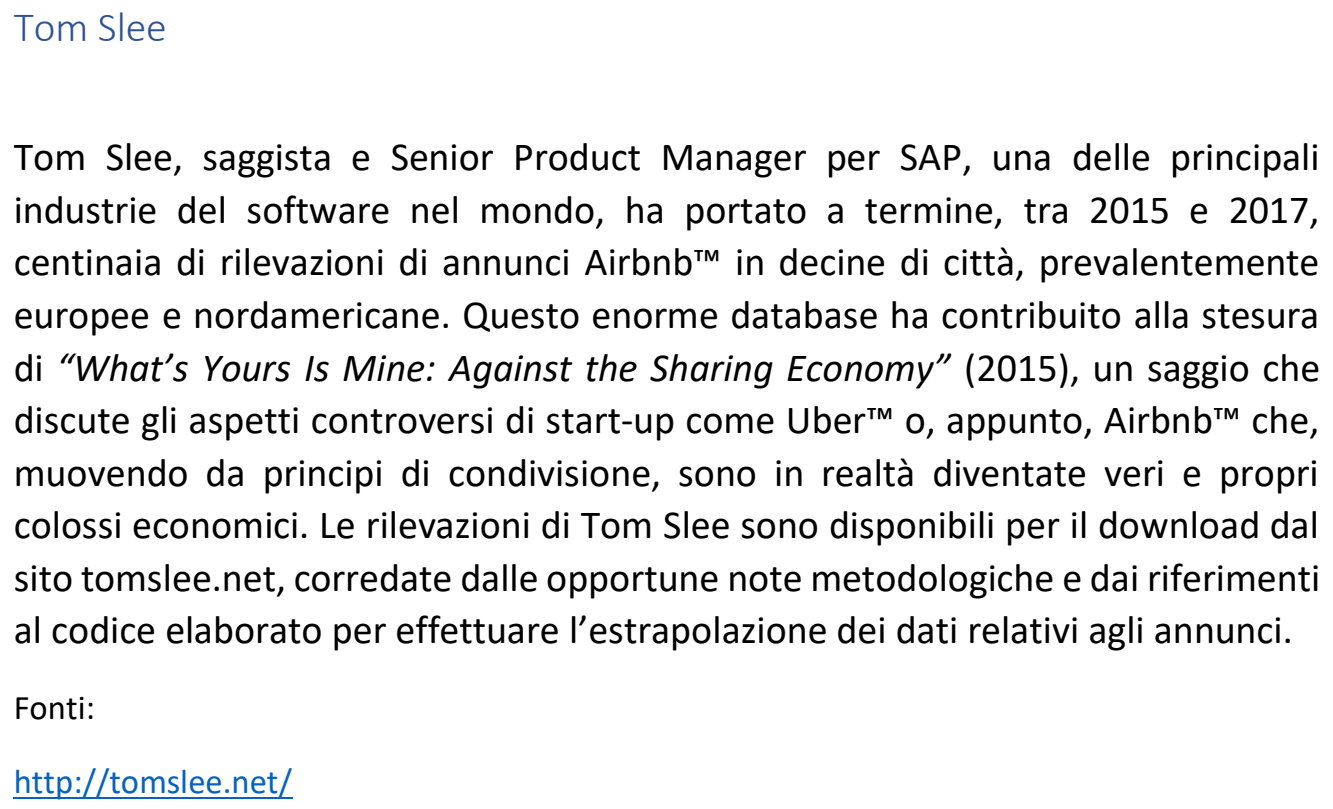
industrie del software nel mondo, ha portato a termine, tra 2015 e 2017, centinaia di rilevazioni di annunci Airbnb ${ }^{\text {TM }}$ in decine di città, prevalentemente europee e nordamericane. Questo enorme database ha contribuito alla stesura di "What's Yours Is Mine: Against the Sharing Economy" (2015), un saggio che discute gli aspetti controversi di start-up come Uber ${ }^{\mathrm{TM}}$ o, appunto, Airbnb ${ }^{\mathrm{TM}}$ che, muovendo da principi di condivisione, sono in realtà diventate veri e propri colossi economici. Le rilevazioni di Tom Slee sono disponibili per il download dal sito tomslee.net, corredate dalle opportune note metodologiche e dai riferimenti al codice elaborato per effettuare l'estrapolazione dei dati relativi agli annunci.

Fonti:

http://tomslee.net/

https://www.theguardian.com/books/2016/apr/02/whats-yours-is-mine-against-thesharing-economy-tom-slee-review

Due sono le rilevazioni complete degli annunci a Bologna svolte dall'autore, il 7 febbraio 2016 e il 30 aprile 2017. In ragione di quanto discusso nel paragrafo precedente, è importante segnalare le date precise perché il meccanismo di funzionamento del portale fa sì che di giorno in giorno gli host possano attivare o disattivare momentaneamente i propri annunci, provocando variazioni nei totali.

Tabella 62. Annunci su Airbnb ${ }^{\text {TM }}$ per Bologna (al 30/4/2017). Distribuzione di frequenza e percentuali per tipologia di abitazione.

\begin{tabular}{|l|c|c|}
\hline 2017 & N & $\%$ \\
\hline Casa intera o appartamento & 1699 & 57.38 \\
\hline Stanza privata & 1201 & 40.56 \\
\hline Stanza condivisa & 61 & 2.06 \\
\hline Totale & 2961 & 100 \\
\hline
\end{tabular}


Utilizzeremo in particolare il database 2017, più recente e coerente con i dati registrati nel medesimo periodo da AirDNA: la Tabella 62 e la Figura 60 ne descrivono la composizione per tipologia di annuncio pubblicato (casa intera, stanza privata o stanza condivisa).

Figura 60. Distribuzione annunci Airbnb ${ }^{\text {TM }}$ per tipologia di abitazione, al 30/4/2017.

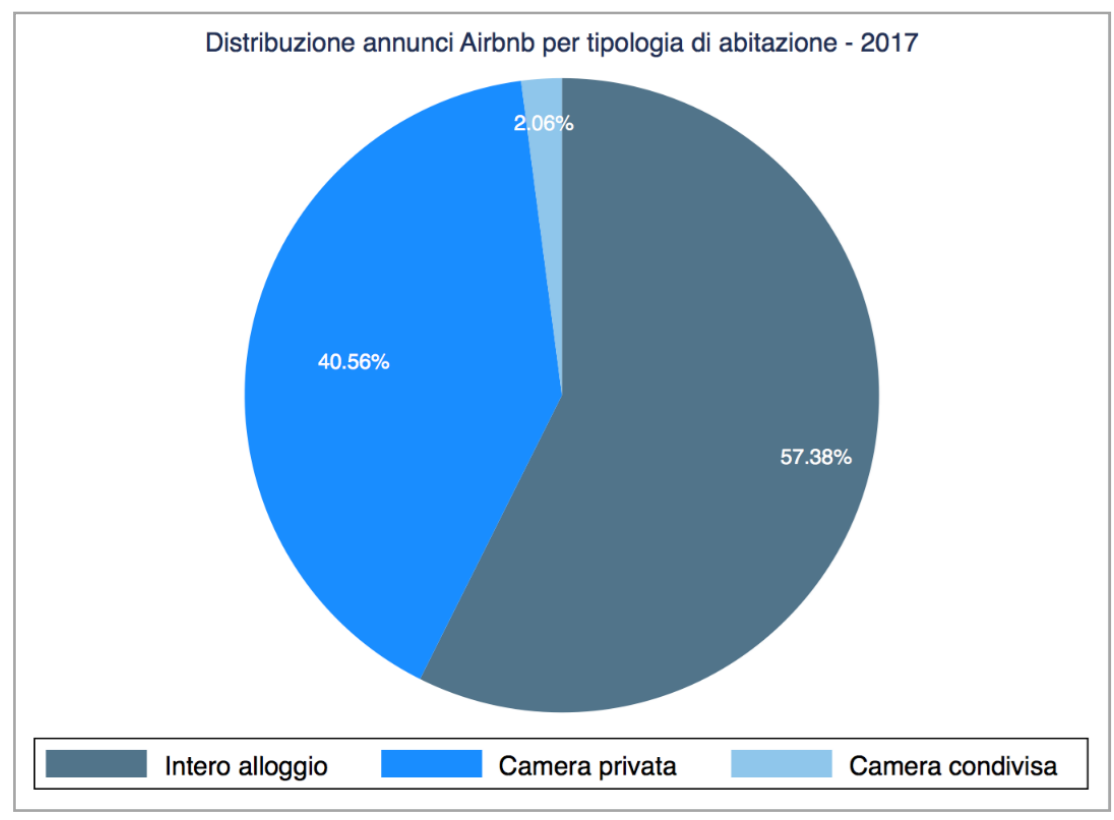

Tra le informazioni aggiuntive registrate dalle rilevazioni di Tom Slee, di ogni annuncio possiamo conoscere numero di camere da letto e capienza. Prima di discuterle, tuttavia, occorre rimarcare ancora una volta che questi immobili sono destinati ad alloggi brevi, che non devono necessariamente rispondere alle medesime esigenze di spazi e vivibilità cui invece è sottoposto il normale mercato locativo per nuclei familiari residenti.

Per questa ragione, e anche per motivi di maggiore attrattività commerciale, ad essere pubblicizzate sul portale di Airbnb ${ }^{\text {TM }}$ come "Casa intera sono spesso monolocali, o piccoli loft e open-space, interamente affittati all'ospite; discrimine dunque non sono tanto le caratteristiche strutturali dell'immobile quanto piuttosto la condivisione o meno dei suoi spazi con altri o con il proprietario stesso.

Non bisogna confondere quindi il numero di camere da letto (la cui distribuzione di frequenza per tipologia di annuncio è riportata nella Tabella 63) con il numero di vani di un'abitazione. A questo proposito, registriamo una netta prevalenza di annunci con una camera da letto, modalità maggioritaria tra le "case intere" e caratteristica peculiare delle restanti tipologie di offerta. Concentrando l'attenzione sulle sole "case intere", solo il $32,7 \%$ di esse hanno due o più camere da letto. 
Tabella 63. Numero di camere da letto per tipologia di annuncio; distribuzione di frequenza e percentuali di riga. Popolazione: annunci Airbnb ${ }^{T M}$ per Bologna al 30/4/2017.

\begin{tabular}{|c|c|c|c|c|c|c|c|c|c|}
\hline \multicolumn{10}{|c|}{ Numero di camere da letto } \\
\hline & & 0 & 1 & 2 & 3 & 4 & 5 & 6 & Totale \\
\hline \multirow{2}{*}{$\begin{array}{l}\text { Casa intera o } \\
\text { appartamento }\end{array}$} & $\mathrm{N}$ & 224 & 1164 & 543 & 111 & 14 & 4 & 2 & 2062 \\
\hline & $\%$ & 10.9 & 56.5 & 26.3 & 5.4 & 0.7 & 0.2 & 0.1 & 100 \\
\hline \multirow[t]{2}{*}{ Stanza privata } & $\mathrm{N}$ & 9 & 1492 & 32 & 6 & 1 & 0 & 0 & 1540 \\
\hline & $\%$ & 0.6 & 96.9 & 2.1 & 0.4 & 0.1 & 0.0 & 0 & 100 \\
\hline \multirow{2}{*}{ Stanza condivisa } & $\mathrm{N}$ & 0 & 93 & 0 & 0 & 0 & 0 & 0 & 93 \\
\hline & $\%$ & 0 & 100 & 0 & 0 & 0 & 0 & 0 & 100 \\
\hline \multirow{2}{*}{ Totale } & $\mathrm{N}$ & 233 & 2749 & 575 & 117 & 15 & 4 & 2 & 3695 \\
\hline & $\%$ & 6.3 & 74.4 & 15.6 & 3.2 & 0.4 & 0.1 & 0.1 & 6.3 \\
\hline
\end{tabular}

La Tabella 64 è invece riferita al numero massimo di posti letto per tipo di alloggio. Ricordando che molti annunci prevedono possibilità di sfruttare a fini di pernottamento spazi ulteriori rispetto alle camere da letto (ad esempio attraverso divani e poltrone letto), non stupisce una capienza media generale superiore alle 3 persone $(3,07)$, benché quattro quinti degli annunci complessivi dispongano al massimo di una camera da letto.

Tabella 64. Numero posti letto massimi per tipologia di annuncio; distribuzione di frequenza e percentuali di riga, media e deviazione standard. La categoria "6 o più" comprende le capienze da 6 a 16 inquilini. Popolazione: annunci Airbnb ${ }^{T M}$ per Bologna al 30/4/2017.

\begin{tabular}{|c|c|c|c|c|c|c|c|c|c|c|}
\hline \multicolumn{11}{|c|}{ Posti letto per annuncio } \\
\hline & & 1 & 2 & 3 & 4 & 5 & $6+$ & Totale & Media & $\begin{array}{l}\text { St. } \\
\text { dev. }\end{array}$ \\
\hline \multirow{2}{*}{$\begin{array}{l}\text { Casa intera } \\
\text { o app.to }\end{array}$} & $\mathrm{N}$ & 10 & 459 & 294 & 545 & 135 & 256 & 1699 & \multirow{2}{*}{3.76} & \multirow{2}{*}{1.66} \\
\hline & $\%$ & 0.6 & 27.0 & 17.3 & 32.1 & 8.0 & 15.1 & 100 & & \\
\hline \multirow{2}{*}{$\begin{array}{l}\text { Stanza } \\
\text { privata }\end{array}$} & $\mathrm{N}$ & 224 & 720 & 158 & 74 & 12 & 13 & 1201 & \multirow{2}{*}{2.14} & \multirow{2}{*}{0.92} \\
\hline & $\%$ & 18.7 & 60.0 & 13.2 & 6.2 & 1.0 & 1.1 & 100 & & \\
\hline \multirow{2}{*}{$\begin{array}{l}\text { Stanza } \\
\text { condivisa }\end{array}$} & $\mathrm{N}$ & 22 & 30 & 3 & 2 & 1 & 3 & 61 & \multirow{2}{*}{2.06} & \multirow{2}{*}{1.47} \\
\hline & $\%$ & 36.1 & 49.2 & 4.9 & 3.3 & 1.6 & 4.9 & 100 & & \\
\hline \multirow{2}{*}{ Totale } & $\mathrm{N}$ & 256 & 1209 & 455 & 621 & 148 & 272 & 2961 & \multirow{2}{*}{3.07} & \multirow{2}{*}{1.61} \\
\hline & $\%$ & 9 & 41 & 15 & 21 & 5 & 9 & 100 & & \\
\hline
\end{tabular}

Tra le "case intere", questa media si alza a 3,76 persone per immobile, con una quota consistente di offerte che consentono l'accoglienza di cinque o più ospiti $(23,1 \%)$; le stanze private e le stanze condivise si confermano modalità di permanenza adatte a una o due persone, per quanto siano presenti in misura minoritaria annunci di quel tipo che garantiscono il pernottamento a gruppi più numerosi.

Alla luce di questa distribuzione di frequenza possiamo stimare la capienza massima degli alloggi Airbnb ${ }^{\mathrm{TM}}$ a Bologna in 9095 visitatori, di cui oltre un terzo ospitati in "case intere" (Tabella 65). 
Tabella 65. Totale persone ospitabili per tipologia di annuncio e fasce di capienza, in alloggi Airbnb $^{\text {TM }}$ a Bologna il 30/4/2017.

\begin{tabular}{|l|r|r|r|r|r|r|r|}
\hline Totale persone ospitabili & \multicolumn{1}{|c|}{$\mathbf{1}$} & \multicolumn{1}{|c|}{$\mathbf{2}$} & \multicolumn{1}{c|}{$\mathbf{4}$} & \multicolumn{1}{c|}{$\mathbf{5}$} & $\mathbf{5}$ & $\mathbf{6 +}$ & Totale \\
\hline Casa intera o appartamento & 10 & 918 & 882 & 2180 & 675 & 1727 & 6392 \\
\hline Stanza privata & 224 & 1440 & 474 & 296 & 60 & 83 & 2577 \\
\hline Stanza condivisa & 22 & 60 & 9 & 8 & 5 & 22 & 126 \\
\hline Totale & & & & & & & 9095 \\
\hline
\end{tabular}

\section{Una mappa degli annunci Airbnb ${ }^{\mathrm{TM}}$ a Bologna}

Il database di Tom Slee, di cui finora abbiamo analizzato la composizione, consente di georeferenziare, e dunque di trasferire su una mappa, ogni annuncio presente a Bologna al momento della rilevazione. Occorre ricordare però quanto già anticipato: Airbnb $^{\text {TM }}$ comunica la collocazione precisa dei suoi alloggi soltanto al momento della conferma della prenotazione, dunque le coordinate che li identificano sul portale possono risultare leggermente sfalsate.

Ciò nonostante, la Figura 61 offre una rappresentazione affidabile della dispersione degli annunci a Bologna al 30 aprile 2017.

Figura 61. Collocazione degli annunci Airbnb ${ }^{T M}$ a Bologna al 30/4/2017. Evidenziate in rosa le aree ospedaliere, in grigio la zona fieristica, tracciate in rosso le linee notturne del trasporto pubblico.

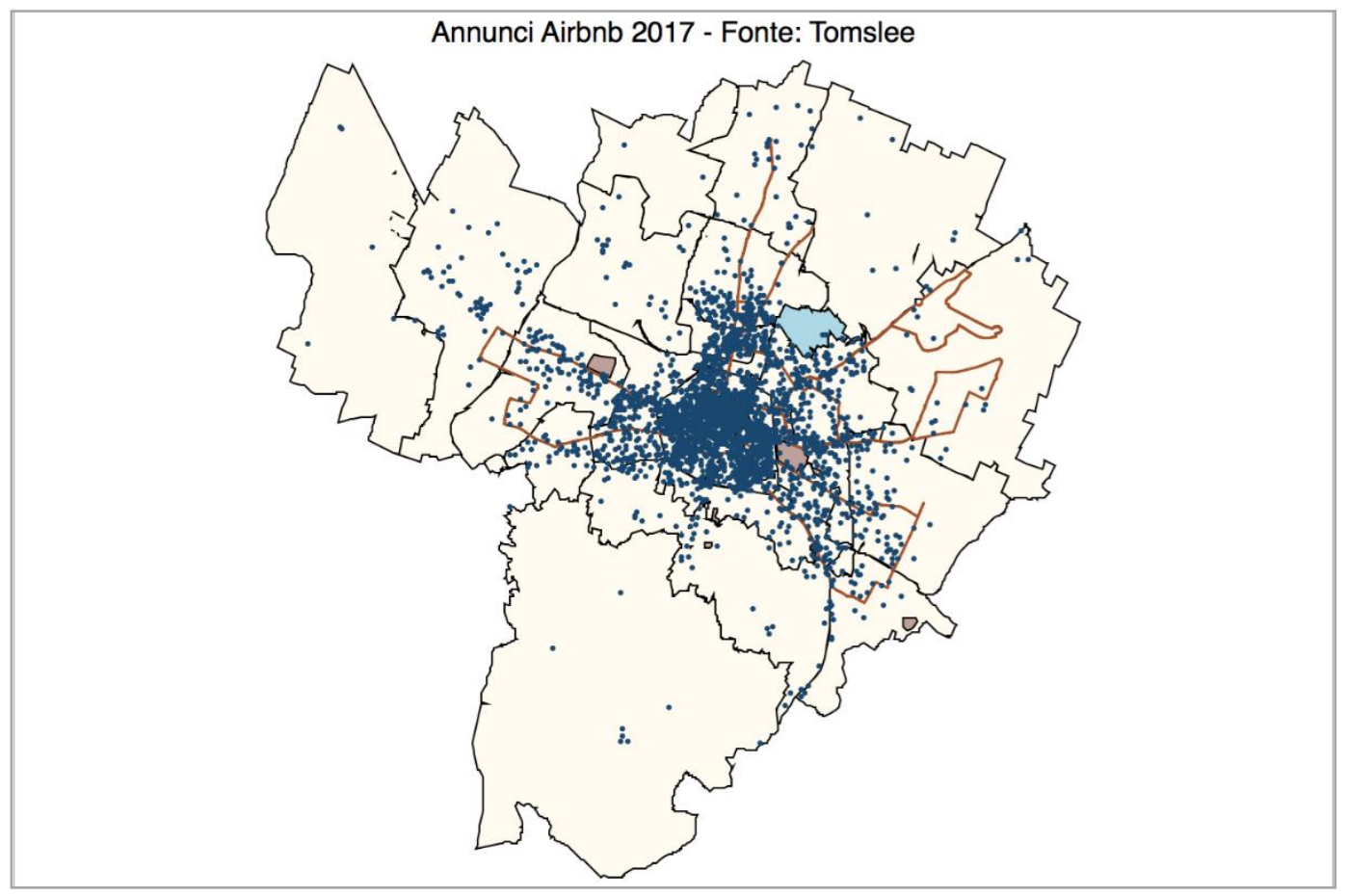


Nella mappa sono state evidenziate le principali aree ospedaliere: troviamo, in ordine da ovest a est, il Maggiore, il Rizzoli, il Sant'Orsola-Malpighi, il Bellaria. A nord est rispetto al centro storico troviamo invece evidenziata l'area fieristica. Alle molteplici ragioni che possono indurre all'alloggio in città, oltre a quelle turistiche e a quelle legate all'Università cui si è fatto cenno in precedenza, possiamo infatti aggiungere la permanenza per assistere un degente nei nosocomi cittadini o la partecipazione ad alcune importanti rassegne organizzate presso la Fiera.

In rosso infine sono tracciate le linee del trasporto pubblico notturno, che consentono ai visitatori di raggiungere i luoghi di interesse del centro anche a serata inoltrata, qualora la sistemazione trovata su Airbnb ${ }^{\text {TM }}$ sia distante da esso.

Oltre all'intero centro storico, dove si concentra la gran parte degli annunci, una discreta densità si osserva anche nelle aree omogenee dell'Osservatorio del Mercato Immobiliare D7, "Matteotti - P.zza Unità", e D8, "Bolognina - Arcoveggio", area densamente popolata e prossima ai padiglioni della fiera.

Discreta concentrazione anche nel settore compreso tra via Emilia Ovest (fino all'Ospedale Maggiore) e la zona Saragozza. Sul lato opposto del centro, le abitazioni si distribuiscono prevalentemente lungo gli assi viari principali, come via San Donato (fino alla Fiera), via Massarenti (in prossimità del Sant'Orsola) e nelle zone D2, D4 e D24 (rispettivamente "San Vitale", "Mazzini-Fossolo", "Murri"). Tutte le aree indicate sono servite dal servizio di trasporto pubblico notturno.

\section{I proprietari degli annunci}

In conformità allo spirito della sharing-economy, Airbnb ${ }^{\mathrm{TM}}$ nasce con l'intento di favorire la condivisione e mettere in contatto viaggiatori e ospiti disponibili ad offrire loro spazi della propria abitazione. Con l'evolversi del mercato, tuttavia, si è sempre più diffusa una gestione "imprenditoriale", attratta dalle potenzialità economiche di questa nuova modalità ricettiva.

Il database di Tom Slee consente di effettuare una preziosa verifica in merito, presentando di ogni annuncio il codice identificativo dell'host, che lo individua univocamente sulla piattaforma e che non può essere cambiato.

Un semplice studio dei duplicati, rispetto al codice identificativo, ha portato in luce la contraddizione tra accoglienza diffusa di iniziativa privata e gestione imprenditoriale.

Soltanto il 55\% del totale è costituito da annunci unici; un ulteriore quarto della popolazione è riconducibile a coloro i quali abbiamo definito "piccoli proprietari", ossia host Airbnb ${ }^{\mathrm{TM}}$ che pubblicano offerte per due o tre alloggi differenti. Oltre 400 soluzioni ricettive (il $14 \%$ del totale) sono attribuibili a "medi proprietari" (da tre a nove annunci diversi); infine, sei "grandi proprietari" si spartiscono il restante $5 \%$, con un picco di 74 abitazioni gestite da Halldis, società di intermediazione immobiliare con oltre 1600 appartamenti in 26 città e luoghi di vacanza in Italia ed Europa. 
Queste società gestiscono gli immobili conferiti loro dai proprietari occupandosi della ricerca degli inquilini e di tutte le fasi dell'accoglienza. Dalle parole di Alberto Melgrati, amministratore delegato di Halldis, raccolte dal Corriere della Sera il 7 dicembre $2016^{87}$, emergono ulteriori dettagli sul funzionamento di questo meccanismo: "il proprietario può stipulare con noi un contratto, annuale o anche di minor durata, con facoltà di recesso in qualsiasi momento e concordare un canone, a forfait $o$, come avviene più spesso, in dipendenza dei giorni di effettiva occupazione dell'immobile. Di norma spese condominiali e utenze restano a carico del proprietario. La società si assume le spese operative, come l'accoglienza, le pulizie, la biancheria e le piccole manutenzioni, e i costi amministrativi e commerciali". Per quanto concerne la parte fiscale, "il cliente ci conferisce un mandato senza rappresentanza grazie al quale possiamo utilizzare l'appartamento come struttura ricettiva extra alberghiera. In questo caso il cliente riceve una cifra che dovrà indicare nelle dichiarazioni fiscali alla voce "altri redditi" in quanto non si tratta più di locazione".

Figura 62. Distribuzione degli annunci Airbnb ${ }^{T M}$ a Bologna al 30/4/2017, per tipo di proprietà. Piccoli proprietari: due o tre annunci; medi proprietari: da tre a nove annunci; grandi proprietari: da dieci annunci.

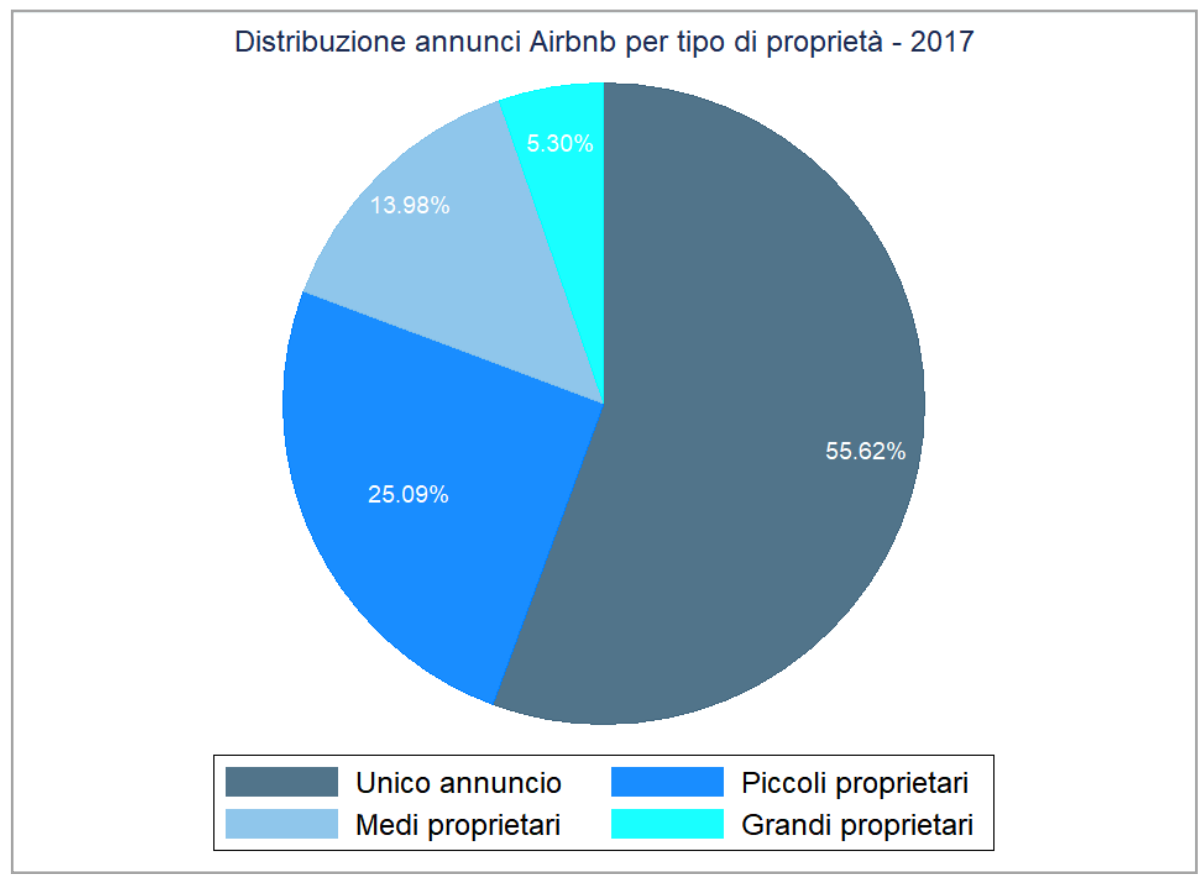

87 "Affitti, non solo Airbnb: Halldis e gli altri gestori «brevi»", Corriere della Sera, 7 dicembre 2016. Link: $\quad$ http://www.corriere.it/economia/finanza e risparmio/16 dicembre 07/affitti-non-soloairbnb-halldis-altri-gestori-brevi-76918bc0-bc98-11e6-9c31-8744dbc4ec0a.shtml 


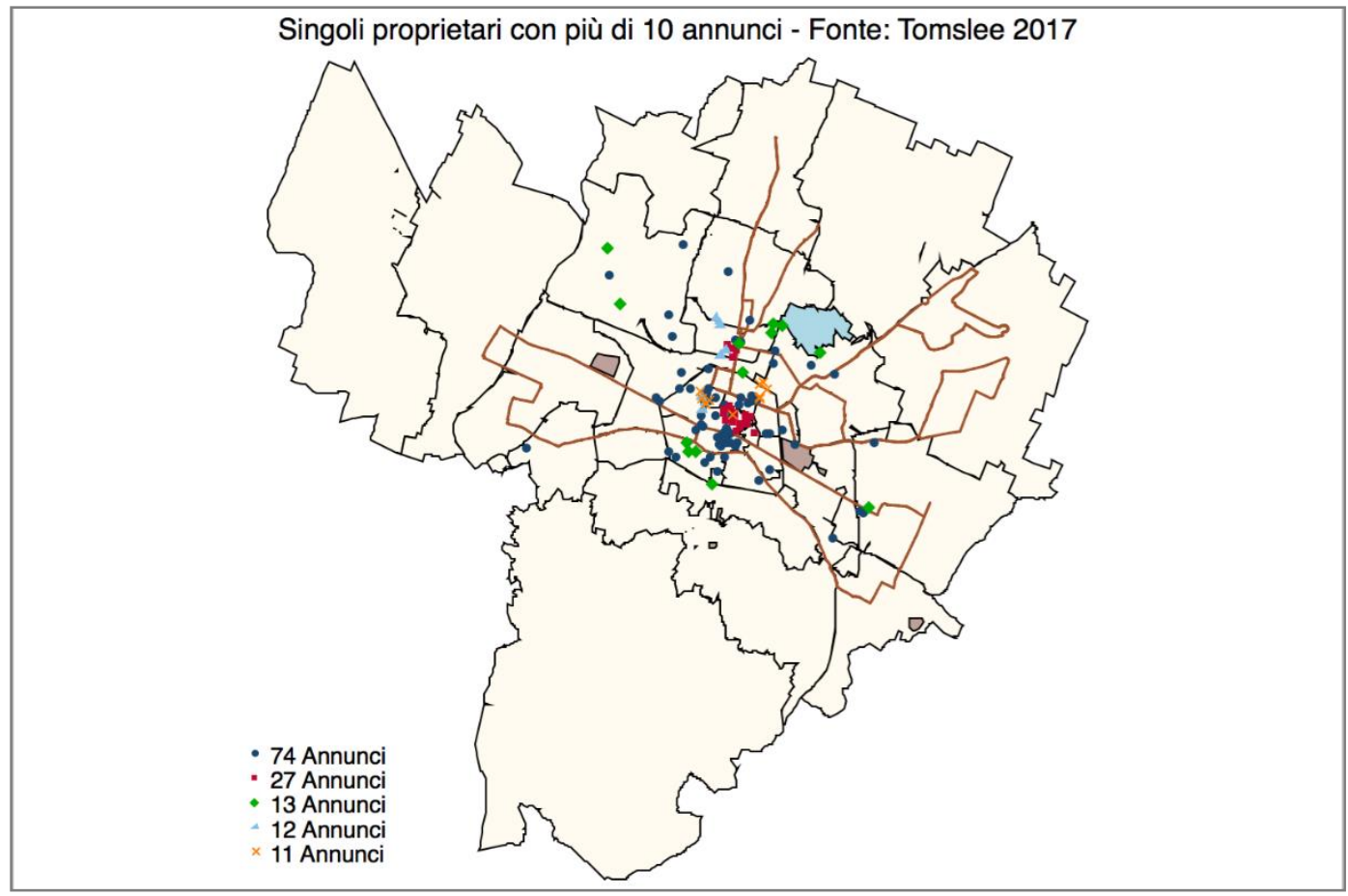

Destinare un immobile all'affitto breve: un'ipotesi sulle entrate.

Benché esista e sia praticata la possibilità di affidare la proprietà ad una società immobiliare che ne curi la promozione, l'affitto e la gestione ordinaria, ricavandone entrate che non si configurano come reddito di locazione, l'accesso al mercato "in proprio" rimane l'opzione prevalente.

Per questo motivo occorre valutare più approfonditamente l'aspetto economico della questione, per indagare la convenienza dell'eventuale spostamento di un immobile dal mercato locativo tradizionale a quello dell'affitto breve, sottraendo in tal modo soluzioni abitative dedicate a famiglie residenti o studenti.

Rispetto allo studio effettuato sulle abitazioni affittate a canone concordato o a canone di mercato, in questo caso occorre fare fronte a più elementi di incertezza nella stima delle entrate per l'host Airbnb' ${ }^{\text {тM }}$ gli annunci possono non essere permanentemente attivi, i prezzi giornalieri possono variare liberamente, esiste una stagionalità (per quanto bassa) del mercato ed un'incertezza rispetto al riempimento dei locali messi a disposizione.

Per poter formulare un'ipotesi, dobbiamo fare affidamento ad entrambi i database utilizzati finora. Delle rilevazioni di Tom Slee utilizzeremo soltanto le 1'699 "case intere", ossia l'unica tipologia di annuncio che potenzialmente potrebbe essere 
destinata a nuclei familiari residenti, considerando il prezzo registrato al 30 aprile 2017.

Per stimare il potenziale incasso annuale, ci gioveremo delle informazioni di AirDNA, traendone le variazioni percentuali dei prezzi rispetto al mese di aprile ed ipotizzando conseguentemente le tariffe mensili. Dalla medesima fonte traiamo l'andamento mensile del tasso di locazione, verificando il livello di riempimento mediano degli annunci. Moltiplicando i prezzi mensili per il rispettivo tasso di locazione (inteso come il numero di giorni locati sul totale dei giorni disponibili) otterremo un'ipotesi di incasso annuale. I dati utilizzati per effettuare le suddette stime sono contenuti nella Tabella 66.

Tabella 66. Prospetto delle variazioni mensili dei prezzi medi giornalieri per "case intere" a Bologna, rispetto ad aprile (\%). Prezzi mensili delle "case intere" contenute nel database Tom Slee, stimati a partire dal prezzo rilevato al 30/4/2017 e dalle relative variazioni mensili. Tasso di locazione mediano e numero di giorni al mese affittati.

\begin{tabular}{|c|c|c|c|c|c|c|c|c|c|c|c|c|}
\hline \multicolumn{13}{|l|}{2017} \\
\hline & ভั & 윤 & $\sum^{\frac{1}{\pi}}$ & 훈 & $\sum^{\infty 00}$ & 忌 & $\stackrel{\circ 0}{3}$ & $\stackrel{\circ}{\stackrel{⿰}{4}}$ & ڤ̆ & Ð゙ & 후 z & 㟧 \\
\hline $\begin{array}{l}\text { Variazione } \\
\text { mensile prezzo } \\
\text { medio (\%) }\end{array}$ & 0.90 & 0.85 & 1.06 & 1.00 & 1.00 & 0.95 & 0.92 & 0.89 & 1.09 & 0.96 & 0.92 & 1.01 \\
\hline $\begin{array}{l}\text { Prezzo } \\
\text { giornaliero } \\
\text { medio nel mese } \\
\text { “Case intere” (€) } \\
\text { - database Tom } \\
\text { Slee }\end{array}$ & 86 & 81 & 101 & 95 & 95 & 90 & 88 & 85 & 104 & 91 & 88 & 96 \\
\hline $\begin{array}{l}\text { Tasso di } \\
\text { locazione } \\
\text { mediano (\%) }\end{array}$ & 38 & 58 & 55 & 73 & 69 & 65 & 58 & 63 & 73 & 74 & 60 & 50 \\
\hline $\begin{array}{l}\text { Numero di giorni } \\
\text { al mese affittati }\end{array}$ & 11.8 & 16.2 & 17 & 21.9 & 21.4 & 19.5 & 18 & 19.5 & 21.9 & 22.9 & 18 & 15.5 \\
\hline
\end{tabular}

Come esplicitato nella Tabella 67, una "casa intera" affittata tramite Airbnb ${ }^{\text {TM }}$ a Bologna, con un tasso di riempimento mediano, genera un incasso annuale medio pari a 19'985 euro, al netto dei costi di gestione trattenuti dal portale (pari al 3\%).

Tabella 67. Incasso annuale medio per tipologia di annuncio.

\begin{tabular}{|l|r|r|r|r|r|}
\hline & \multicolumn{5}{|c|}{ Incasso annuale medio per tipologia annuncio } \\
\hline & 0 camere & 1 camera & 2 camere & 3 o più camere & Totale \\
\hline Incasso annuale medio (€) & 14449 & 17926 & 21564 & 38376 & 19985 \\
\hline $\mathbf{N}$ & 173 & 949 & 458 & 119 & 1699 \\
\hline
\end{tabular}


Non siamo in grado di fornire un dettaglio dei prezzi su base geografica, ad esempio per zona di applicazione del canone concordato, a causa delle lievi imprecisioni nelle coordinate fornite da Airbnb ${ }^{\mathrm{TM}}$, che non consentono una precisa collocazione dei singoli annunci. É invece più interessante osservare come vari l'incasso annuale medio in base al numero di camere da letto presenti nell'alloggio, al crescere del quale I'incasso annuale medio sale da 14'449 euro a 38'376, a testimonianza di una grande variabilità attorno alla media.

Gli incassi potenziali evidenziati sono estremamente alti, pur con un tasso di riempimento mediano; occorre raffrontarli con quelli generati dai tradizionali contratti a canone concordato, per valutare quanto possa essere conveniente per un proprietario destinare la propria "casa intera" al mercato del soggiorno turistico breve.

Ai fini di questo confronto applichiamo agli incassi da Airbnb ${ }^{\mathrm{TM}}$ la cedolare secca da entrate da locazioni al $21 \%$, per quanto, come detto, sia aperta anche la possibilità di sottoporre tali introiti al regime Irpef.

Per quanto concerne il mercato a canone concordato utilizziamo gli affitti medi calcolati sugli immobili locati a canone concordato nel 2014. Per uniformità, suddivideremo le abitazioni incluse in quello stock come segue:

- I monolocali corrisponderanno a "0 camere da letto" nella popolazione Airbnb ${ }^{\mathrm{TM}}$

- I bilocali corrisponderanno a "1 camera"

- I trilocali corrisponderanno a "2 camere"

- Le abitazioni con un numero di vani pari o superiore a 4 corrisponderanno a "3 o più camere".

Occorre inoltre segnalare che la collocazione all'interno delle diverse zone di applicazione del canone concordato è una variabile determinante nella definizione degli affitti mensili; non potendo però attribuire con certezza gli annunci Airbnb ${ }^{\mathrm{TM}}$ alle medesime zone, la discriminante geografica non verrà considerata.

Tabella 68. Incasso annuale medio netto per tipologia di abitazione: Airbnb ${ }^{\text {TM }}$ e canone concordato.

\begin{tabular}{|c|c|c|c|c|}
\hline & $\begin{array}{c}0 \\
\text { camere }\end{array}$ & $\begin{array}{c}1 \\
\text { camera }\end{array}$ & $\begin{array}{c}2 \\
\text { camere }\end{array}$ & $\begin{array}{c}3+ \\
\text { camere }\end{array}$ \\
\hline Incasso annuale medio (€) Airbnb ${ }^{\mathrm{TM}}$ & 11415 & 14162 & 17036 & 30317 \\
\hline Incasso annuale medio $(€)$ Canone concordato & 3675 & 4122 & 4515 & 6068 \\
\hline
\end{tabular}

La Tabella 68 riporta il consistente divario tra le due modalità di affitto, in termini di entrate. Annualmente un monolocale su Airbnb ${ }^{\mathrm{TM}}$, senza nemmeno dover registrare presenze di ospiti al di sopra della media, produce quasi tre volte di più di quanto 
possa fare sul mercato del canone concordato, e tale proporzione cresce progressivamente fino al rapporto di cinque a uno registrato per le abitazioni con tre o più vani. Ciò peraltro avviene al netto di due aliquote diverse: per i contratti a canone, infatti, la cedolare secca si attesta al $10 \%$, mentre per le locazioni sul libero mercato (tra cui, ovviamente, gli affitti brevi Airbnb ${ }^{\mathrm{TM}}$ ) raggiunge il $21 \%$.

Questa disparità di prospettive economiche si verifica anche considerando i valori degli affitti a canone concordato per la sola Zona di Pregio (la più costosa) o prendendo a paragone i prezzi massimi. In altri termini, nonostante la consapevolezza che il confronto possa risultare leggermente forzato per le diverse necessità cui deve assolvere un alloggio adibito a brevi soggiorni rispetto all'abitazione principale e nonostante il fatto che le locazioni a turisti lascino in carico al proprietario il pagamento delle utenze (cosa che solitamente non avviene per gli affitti lunghi), l'esplosione del mercato degli affitti brevi Airbnb ${ }^{\mathrm{TM}}$ pone questioni serie al mercato locativo tradizionale. Al momento, infatti, per un proprietario di un immobile iscriversi come host al portale è estremamente vantaggioso da più punti di vista:

- Quello delle entrate innanzitutto: anche un afflusso di ospiti decisamente al di sotto rispetto al tasso di riempimento medio è sufficiente a superare l'incasso garantito da un normale contratto a canone concordato o di mercato.

- La facoltà di disattivare e riattivare l'annuncio a piacimento offre il pieno controllo dell'immobile e la possibilità di tornarne nella sua piena disponibilità in qualunque momento.

- Un utilizzo meno intensivo dell'immobile porta, salvo eccezioni, ad un minore logoramento dello stesso.

- "Provarci" è un'opzione a quasi totale assenza di rischi: non esistono costi di ingresso a parte eventuali risistemazioni dello stabile e degli arredi (che ne accrescono comunque il valore).

É lecito immaginare che la crescita del mercato cui stiamo assistendo possa fisiologicamente giungere, nel tempo, ad un assestamento. Alle condizioni attuali, tuttavia, si può in via prudenziale prevedere (trattasi comunque di una primissima analisi del fenomeno a Bologna) che il trasferimento di abitazioni dal mercato tradizionale ad Airbnb ${ }^{\text {TM }}$ possa proseguire.

\section{Una prospettiva sul mercato}

Quante abitazioni ancora potranno essere sottratte al mercato residenziale, stanti le condizioni attuali, prima che l'opzione dell'affitto su Airbnb ${ }^{\text {TM }}$ cessi di essere vantaggiosa rispetto ad un comune contratto a canone di mercato? Al momento non disponiamo di dati sufficientemente dettagliati per indagare fino a che punto questo fenomeno possa espandersi nel futuro. In attesa di poterci cimentare con un'analisi più raffinata, è comunque possibile prevedere uno scenario 
di base. A tal fine alcune ipotesi sono necessarie: innanzitutto è necessaria una stabilizzazione dei flussi turistici su cui si fondano i dati registrati nei database AirDNA. L'ipotesi è estremamente prudenziale (per non dire conservativa) al fine di fornire un primo conteggio a valori acquisiti: come detto ad inizio capitolo, infatti, le presenze di turisti a Bologna sono in crescita costante da anni. In secondo luogo si effettua la previsione per le sole "case intere", l'unica tipologia di annunci sul portale che effettivamente porta alla sottrazione di un'intera unità abitativa al mercato locativo tradizionale. In questo contesto infatti non consideriamo il vantaggio di mettere in opera complete ristrutturazioni immobiliari atte ad ottenere differenti unità abitative (mono e bilocali) da case di maggiore dimensione data la complessità e il costo dell'intervento.

Come abbiamo appena constatato, una "casa intera", con un tasso di riempimento mediano, e un prezzo di pernottamento medio, genera un'entrata annuale di 19'985 euro lordi, ossia (89,6 $€$ per 223,7 giorni pernottati, si vedano le precedenti Tabella 66 e Tabella 67.

Dopo aver verificato la netta superiorità della scelta di affitto turistico breve rispetto ai profitti generati da un contratto a canone concordato medio, prendiamo a paragone le entrate generate da un contratto di affitto a canone di mercato calcolato nei suoi valori massimi ${ }^{88}$. Un termine di confronto, questo, giustificato dal fatto che le "case intere" affittate su Airbnb ${ }^{\mathrm{TM}}$ siano generalmente in ottimo stato e collocate in zone di valore, dunque potenzialmente applicherebbero sul mercato prezzi al metro quadro più alti della media. Attualmente, in media, le entrate annuali lorde di un contratto a canone di mercato, definito nei suoi parametri massimi, sono 8340 euro (risultanti da un affitto mensile pari a 695,6 euro).

Tabella 69. Prospetto dei dati utilizzati nella simulazione del potenziale di espansione dell'offerta Airbnb ${ }^{\text {TM }}$ a flusso turistico costante.

\begin{tabular}{l|r|}
\hline $\begin{array}{l}\text { Prospetto dati simulazione potenziale espansione dell’offerta Airbnb } \\
\text { turistico costante }\end{array}$ & $19985 €$ \\
\hline $\begin{array}{l}\text { Entrate annuali annunci “casa intera" Airbnb } \\
\text { mediano) }\end{array}$ & 223,7 \\
\hline Numero giorni pernottati (tasso di occupazione mediano) & $89,6 €$ \\
\hline Costo unitario pernottamento & $80.4 €$ \\
\hline Costo unitario pernottamento scontato da spese di gestione (-10\%) & $8340 €$ \\
\hline Entrate annuali contratto a canone di mercato, parametri al metro quadro massimi & 103,7 \\
\hline Numero giorni pernottati su Airbnb & per raggiungere entrate mercato \\
\hline Potenziale espansione offerta Airbnb & 2,157 \\
\hline
\end{tabular}

88 Questo dato è calcolato applicando i parametri OMI massimi per il 2017 ad ogni alloggio del nostro stock 2014, moltiplicandoli per la rispettiva superficie ed infine definendo il valore medio tra i canoni calcolati per ognuna. 
Nel confronto tra le due modalità di locazione, teniamo prudenzialmente conto delle spese di gestione quotidiana che un affitto breve comporta, stimando che le entrate Airbnb $^{\text {TM }}$ lorde effettive siano inferiori del 10\%, dunque 17'987 euro annui, ossia 80,4€ per ogni giorno pernottato.

I conti sono presto fatti: per guadagnare tramite Airbnb ${ }^{\mathrm{TM}}$ quanto si guadagna con un contratto a canone di mercato (8'340 euro), è sufficiente affittare il proprio appartamento 104 giorni all'anno. Perché a parità di flussi turistici, dunque di domanda di alloggi, cali così sensibilmente il tasso di occupazione (da 223,7 a 104 giorni pernottati), non può che avvenire un aumento dell'offerta. In altri termini: alle condizioni attuali, il numero di "case intere" affittate su Airbnb ${ }^{\mathrm{TM}}$ potrebbe raddoppiare continuando a garantire ai proprietari che si inseriscono in questo settore entrate superiori a quanto genererebbe un contratto locativo di mercato nei suoi valori massimi.

Se a fine aprile 2017, in accordo con il database di Tom Slee, le "case intere" in offerta sul portale erano 1'699, potremmo orientativamente aspettarci che circa altrettante possano essere sottratte al mercato residenziale. Un dato già di per sé piuttosto importante, che potrebbe assumere in realtà proporzioni maggiori dal momento che la tendenza e le aspettative sull'andamento delle presenze turistiche prevedono un ulteriore incremento degli arrivi. 


\section{Conclusione all'analisi quantitativa}

In questa sessione si ripropongono alcuni dei punti fondamentali già esplicitati in introduzione all'analisi quantitativa.

Nello specifico, secondo le stime effettuate sulla base del censimento ISTAT 2011 e dei dati catastali relativi allo stesso anno, il mercato locativo bolognese è caratterizzato da un potenziale eccesso di domanda di circa 6'000 famiglie cui non corrisponde un'allocazione economicamente sostenibile o qualitativamente adeguata nel mercato locativo bolognese. ${ }^{89}$ Per quanto questi conti siano basati su numerose ipotesi, risulta evidente come tale mercato sia caratterizzato da una domanda sostenuta, determinata da un maggior numero di persone in cerca di affitto rispetto al totale delle abitazioni offerte sul mercato.

In termini numerici il canone concordato appare essere la principale forma di contratto con una forte crescita dopo la variazione (abbassamento al 10\%) della cedolare secca del 2013. In termini numerici si dispone di 20'988 alloggi nel 2010, 20'588 nel 2012 e 25'345 nel 2014 di dimensione superiore ai 30mq. L'incidenza di tale forma contrattuale è decrescente più ci si allontana dal centro. Inoltre la presenza di alloggi a canone concordato è preponderante nelle metrature medio-piccole (dove per altro il canone concordato a volte supera la media del mercato) mentre si ha scarsa disponibilità di edifici di metratura superiore e numero di vani superiore.

II 2013, con la riforma della tassazione sui redditi derivanti da locazione immobiliare, nota come "cedolare secca", è stato l'anno di svolta per questa forma contrattuale e in generale per il mercato locativo bolognese. I nuovi accordi sul canone concordato hanno sancito un calo vicino al $10 \%$ dei valori al metro quadro, più sensibile nei parametri massimi. Ancora più intenso il calo dei prezzi di mercato: nel 2017, al termine della serie storica considerata, i valori risultano decisamente inferiori rispetto al 2009: -16\% per i minimi e -22\% per i massimi.

Quando si valuta la sostenibilità per il nucleo mediano bolognese, fatte le dovute considerazioni sulle assunzioni necessarie (l'affitto non deve superare il $30 \%$ del reddito complessivo), il canone concordato risulta alla sua portata nella Zona $\mathrm{B}$ e, anche se in misura minore, nella Zona $A$; più complessa la situazione nella costosa Zona di Pregio, mentre paiono chiudersi molte porte per i nuclei mono-personali (o monoreddito), costretti a spingersi in periferia.

La combinazione di concorrenzialità e sostenibilità determina diverse categorie all'interno delle quali sono classificate le abitazioni della banca dati. Il numero di abitazioni sostenibili e concorrenziali, e quindi effettivamente locabili a canone concordato, aumenta nel 2014 rispetto agli anni precedenti attestandosi al 25\% circa dell'intero stock. Queste abitazioni, però, sono perlopiù di dimensioni ridotte

${ }^{89}$ Si veda pagina 51 per dettagli sul concetto di eccesso di domanda. 
(superficie media $52.18 \mathrm{mq}$ e numero medio di vani 1.76, rispetto alle medie cittadine di circa $80 \mathrm{mq}$ e 3.32 vani - valori 2014) e non adatte, quindi, alle famiglie più numerose.

Analizzando questo aspetto anche all'interno delle singole zone di applicazione del canone concordato è possibile dimostrare come, sempre con riferimento al 2014, la percentuale di abitazioni ottimali sia maggiore per la zona di pregio (31.42\%) rispetto alla zona $\mathrm{A}(28.25 \%)$ e alla $\mathrm{B}(20.31 \%)$. Le percentuali più basse registrate per le zone $A$ e $B$ sono determinate dalla minor concorrenzialità, come dimostrano le quote delle categorie residue (le abitazioni sostenibili ma non concorrenziali aumentano passando da zona di pregio a zona B).

L'offerta del mercato del canone concordato bolognese è inadatta a soddisfare le esigenze dei nuclei con redditi molto bassi e la causa principale va ricercata nella sostenibilità: da un lato i redditi di queste famiglie sono troppo bassi, dall'altro i canoni di locazione sono troppo elevati. Se in termini economici per altri beni tale problema potrebbe essere marginale, la casa, assieme all'alimentazione, rientra nei beni fondamentali per la sopravvivenza e quindi il dato appare particolarmente allarmante. A concorrere alla produzione di questo contesto vi sono da sempre gli studenti fuori sede e in tempi recenti il fenomeno dell'affitto turistico a breve termine.

Assunto che ogni studente paga in media $350 €$ per una camera singola in appartamento condiviso, un proprietario potrà applicare i prezzi al metro quadro più alti consentiti dal canone, che in Zona di Pregio non sarebbero sostenibili dalle famiglie, trovando inesauribile e redditizia risposta al proprio annuncio di locazione. Questo meccanismo di potenziale esclusione dei residenti, aggravato da potenziali pratiche illecite (l'utilizzo dei canoni concordati per coprire una cospicua parte in nero o di sub-affitto), e alle recenti innovazioni nel calcolo delle tasse universitarie, fanno di questo mercato un fenomeno da monitorare approfonditamente.

Destinare un'abitazione al mercato dell'affitto turistico a breve termine, sottraendola da quelle disponibili per normali affitti residenziali, è decisamente più conveniente, non solo per la flessibilità nella gestione degli annunci (disattivabili a piacimento e senza costi di entrata), ma soprattutto per gli enormi ricavi se paragonati all'affitto a lungo periodo. Nel 2017 una "casa intera" con un prezzo medio e occupata per 232 giorni l'anno (dato mediano per la città) può arrivare a fruttare, al netto delle tasse, da tre a cinque volte un affitto medio a canone concordato. Margini talmente ampi da consentire, a parità di flussi turistici, un trasferimento di oltre 1'700 ulteriori alloggi dal mercato residenziale a quello turistico, garantendo comunque a tutti gli affittuari Airbnb $^{\text {TM }}$ entrate superiori al massimo ottenibile con un normale contratto d'affitto a canone di mercato. 


\section{Riflessioni conclusive}

Questa ricerca è stata un primo, preliminare tentativo di quantificare la domanda e l'offerta di abitazioni a locazione nel territorio comunale bolognese. Scopo della ricerca è stato tracciare una prima via di analisi che permettesse di identificare $i$ principali meccanismi che spingono i proprietari ad allocare sul mercato dell'offerta locativa i propri immobili.

All'interno di un contesto in cui esistono interessi divergenti dal punto di vista della domanda di abitazione in locazione, sebbene l'analisi sia basata su informazioni ancora parziali e che richiedono maggiore dettaglio, si sono ottenute alcune prime indicazioni:

1. Bologna presenta una forte domanda di unità abitative in locazione; tale domanda è stabile quando non crescente.

2. Bologna presenta una offerta abitativa a locazione che deve soddisfare due domande in forte concorrenza fra loro, quella familiare e quella studentesca.

3. Negli ultimi due anni i successi sul fronte della crescita del turismo cittadino hanno generato una nuova, profittevole, forma di utilizzo delle unità abitative stesse.

A fronte di queste condizioni appare chiaro nell'analisi che, nonostante assunzioni molto prudenziali, ad oggi vi sia "un'eccesso di domanda" da parte delle famiglie nell'ordine delle 6000 unità. Questo si riflette in prezzi di equilibrio di mercato particolarmente elevati, spesso non sostenibili da parte delle famiglie mediane bolognesi e quasi mai sostenibili dalle famiglie in difficoltà economica.

Il mercato dell'affitto breve per motivi turistici ha già assorbito 1700 unità e probabilmente nei prossimi anni ne assorbirà almeno altrettante, contribuendo ad aumentare ulteriormente i prezzi o quantomeno a ridurre l'offerta.

Sebbene i risultati siano preliminari, risulta evidente come il policy maker sia di fronte ad una situazione che richiede forme di intervento. Nonostante questa ricerca sia a uno stadio troppo preliminare per proporre soluzioni specifiche, alcune proposte possono essere derivate:

I. L'accesso al canone concordato da parte degli studenti risulta per i locatari una strategia dominante quindi tendente a traslare uno strumento ideato per favorire la locazione delle famiglie verso soggetti differenti - si ritiene che rimodulare le agevolazioni in favore delle famiglie potrebbe portare benefici laddove l'obbiettivo sia favorire la locazione a famiglie;

II. La Zona B del canone concordato appare troppo estesa ed eterogenea, in questo modo i prezzi uguali rischiano di ridurre l'offerta a canone concordato nelle zone più attrattive ed economicamente relativamente benestanti in favore del canone libero. 
III. Aumentare l'offerta locativa a Bologna attraverso il recupero dello sfitto (che tuttavia in questa analisi apparirebbe essere relativamente ridotto ma richiede ulteriore indagine) o il recupero di aree e immobili dismessi.

IV. La questione studentesca appare centrale, il dislocamento di parte della domanda abitativa dei cosiddetti "fuorisede" non è al centro della ricerca qui proposta ma appare come una possibile soluzione da valutare - più in generale la carente offerta di alternative in termini di studentati o di aree abitatitve di interesse per gli studenti richiede un focus specifico.

V. II fenomeno dell'affitto turistico a breve richiede un supplemento di indagine per calcolare benefici individuali e di comunità.

VI. La questione abitativa non appare confinabile nel solo territorio del Comune di Bologna ma riguarda chiaramente anche le aree limitrofe all'interno della Città Metropolitana. 


\section{Bibliografia}

Agenzia delle entrae. (2012). Statistiche catastali 2011. Tratto da: https://wwwt.agenziaentrate.gov.it/mt/osservatorio/Tabelle\%20statistiche/archivio/Statistiche_Catastali_201 1.pdf

Agenzia metropolitana per l'affitto (AMA). Tratto da http://www.ama.bo.it/1/1/il-progetto

Airbnb. Chi può ospitare con Airbnb? Tratto da https://www.airbnb.it/help/article/18/who-can-host-on-airbnb

Airbnb. Ospitare responsabilmente in Italia. Tratto da https://www.airbnb.it/help/article/1394/ospitareresponsabilmente-in-italia

Airdna. Tratto da www.airdna.co

Alma Mater Studiorum Università di Bologna. Alloggi e residenze a Bologna. Tratto da www.unibo.it/it/servizi-eopportunita/borse-di-studio-e-agevolazioni/alloggi-e-sportello-registrazione-affitti/alloggi-e-residenze-abologna/alloggi-e-residenze-a-bologna

Alma Mater Studiorum Università di Bologna. Costo della vita a Bologna. Tratto da http://www.unibo.it/it/servizi-eopportunita/studio-e-non-solo/vivere-in-citta/costo-della-vita-a-bologna

Alma Mater Studiorum Universtà di Bologna. Bologna, città dei collegi. Tratto da http://www.unibo.it/it/servizi-eopportunita/borse-di-studio-e-agevolazioni/alloggi-e-sportello-registrazione-affitti/alloggi-e-residenze-abologna/bologna-citta-dei-collegi-1

Assoaeroporti. Statistiche aeroportuali Dicembre 2017. Tratto da www.assaeroporti.com/statistiche_201712/

Azienda regionale per il diritto agli studi superiori (ER.GO). Tratto da www.er-go.it

Baldini, M. (2009). Quaderni della ricerca sociale 22 - Le politiche sociali per la casa in Italia. Tratto da Ministero del lavoro e delle politiche sociali: http://sitiarcheologici.lavoro.gov.it/Strumenti/StudiStatistiche/Documents/QRS22_casa.pdf

Baldini, M., \& Federici, M. (2011, March). Ethnic discrimination in the Italian rental housing market. Journal of Housing Economics, 20(1), 1-14.

Baldini, M., \& Poggio, T. (2009). Le politiche rivolte all'affitto e i loro effetti. In A. Brandolini, C. Saraceno, \& A. Schizzerotto, Dimensioni della diseguaglianza in italia: povertà, salute, abitazione. II Mulino.

Baldini, M., \& Poggio, T. (2012). Housing Policy Towards the Rental Sector in Italy: A Distributive Assessment. Housing Studies, 27(5), 563-581.

Banca d'Italia. (2014, Gennaio 27). Supplementi al bollettino statistico - I bilanci delle famiglie italiane nell'anno 2012. Tratto da https://www.bancaditalia.it/pubblicazioni/indagine-famiglie/bil-fam2012/suppl_05_14n.pdf

BolognaToday. (2016, Febbraio 23). Affitto case "in nero" a centinaia di studenti, stanato agente immobiliare abusivo. Tratto da http://www.bolognatoday.it/economia/case-affitto-nero-bologna-agente-immobiliare-abusivo.html

Business Insider. (2017, Agosto 10). Airbnb now has more listings worldwide than the top five hotel brands combined. Tratto da http://www.businessinsider.com/airbnb-total-worldwide-listings-2017-8?IR=T

Città Metropolitana di Bologna. (2014, Giugno 23). Accordo applicabile nel Comune di Bologna per i contratti d'affitto a canone concordato. Tratto da http://www.cittametropolitana.bo.it/pianificazione/Engine/RAServeFile.php/f/politiche_abitative/Accordo_ca none_concordato.pdf

Comune di Bologna. (2017, Ottobre 25). Imposta di soggiorno, con il Comune per gli host di Airbnb è tutto più semplice. Tratto da http://comunicatistampa.comune.bologna.it/2017/imposta-di-soggiorno-con-il-comune-per-gli-hostdi-airbnb-e-tutto-piu-semplice 
Corriere della sera. (2016, Dicembre 7). Affitti, non solo Airbnb: Halldis e gli altri gestori "brevi". Tratto da http://www.corriere.it/economia/finanza_e_risparmio/16_dicembre_07/affitti-non-solo-airbnb-halldis-altrigestori-brevi-76918bc0-bc98-11e6-9c31-8744dbc4ec0a.shtml

Corriere di Bologna. (2018, Febbraio 8). Bologna, I'Alma Mater taglia le tasse agli studenti universitari. Tratto da http://corrieredibologna.corriere.it/bologna/cronaca/18_febbraio_08/02-bolognadocumentoocorrierebologna-web-bologna-15c45d34-0ca3-11e8-9d55-099ca8cadaf4.shtml

Di Ciaccio, A., \& Borra, S. (2008). Statistica-metodologia per le scienze economiche e sociali. McGraw-Hill.

Gal, J. (2010, October 27). Is there an extended family of Mediterranean welfare states? Journal of European Social Policy, $20(4), 283-300$.

Gazzetta Ufficiale. (2011, Maggio 23). Codice del turismo - Decreto legislativo n. 79/2011.

I.Stat. (2017). Tratto da Istituto Nazionale di Statistica (ISTAT): http://dati.istat.it/

Il fatto quotidiano, Emilia Romagna. (2014, Febbraio 28). Affitti in nero, famiglia proprietaria di 117 immobili evade 3 milioni di euro. Tratto da https://www.ilfattoquotidiano.it/2014/02/28/affitti-in-nero-famiglia-proprietaria-di117-immobili-evade-3-milioni-di-euro/897138/

Iperbole, Comune di Bologna. (2016, Settembre 27). Tornano i bus notturni tra centro e periferie nei fine settimana. Tratto da www.comune.bologna.it/news/tornano-i-bus-notturni-tra-centro-e-periferie-nei-fine-settimana

ISTAT. (2011). $15^{\circ}$ Censimento della popolazione e delle abitazioni. Tratto da http://www.istat.it/it/censimentipermanenti/censimenti-precedenti/popolazione-e-abitazioni/popolazione-2011

Kemp, P. A. (2011). Low-income Tenants in the Private Rental Housing Market. Housing Studies, 26(7-8), 1019-1034.

La repubblica, Bologna. (2018, Febbraio 7). Ateneo di Bologna, a 20mila studenti l'esonero totale dalle tasse. Tratto da http://bologna.repubblica.it/cronaca/2018/02/07/news/unibo-188276090/

La Republica, Bologna. (2017, Febbraio 19). Bologna, boom di turisti: 2,4 milioni nel 2016. Tratto da bologna.repubblica.it/cronaca/2017/02/19/news/bologna_boom_di_turisti_2_4_milioni_nel_2016$158661833 /$

L'aria d'estate, La7, Lo Muzio, V. (2016, Agosto 31). Bologna, la giungla degli affitti in nero agli studenti. Tratto da http://www.la7.it/lariadestate/video/bologna-la-giungla-degli-affitti-in-nero-agli-studenti-31-08-2016-192228

Newbold, P., Carlson, W., \& Thorne, B. (2010). Statistica. Pearson.

OpenData BETA. Tratto da Comune di Bologna: http://dati.comune.bologna.it/dati

Osservatorio del mercato immobiliare (OMI). Manuale della Banca Dati Quotazioni dell'Osservatorio del Mercato Immobiliare - Istruzioni tecniche per la formazione della Banca Dati Quotazioni OMI. Tratto da Agenzia delle entrate:

http://www.agenziaentrate.gov.it/wps/file/Nsilib/Nsi/Schede/FabbricatiTerreni/omi/Manuali+e+guide/omiall egati/AGENZIA+DELLE+ENTRATE_Manuale+BDQ_OMI_2016_ALLEGATI.pdf

Osservatorio del mercato immobiliare (OMI). Quotazioni immobiliari. Tratto da Agenzia delle entrate: http://www.agenziaentrate.gov.it/wps/content/nsilib/nsi/schede/fabbricatiterreni/omi/banche+dati/quotazio ni+immobiliari

Poggio, T. (2009). Le principali dimensioni della disuguaglianza abitativa in Italia. In A. Brandolini, C. Saraceno, \& A. Schizzerotto, Dimensioni della disuguaglianza in Italia: povertà, salute, abitazione. II Mulino.

Provincia di Bologna. (2008, Febbraio 7). Accordo applicabile nel Comune di Bologna per i contratti d'affitto a canone concordato. Tratto da http://emiliaromagna.agenziaentrate.it/sites/emiliaromagna/files/private/documenti/Locazioni/accordo_affitt o_Bologna.pdf

Regione Emilia Romagna. (2004, Luglio 28). Legge regionale n.16/2004. Tratto da http://demetra.regione.emiliaromagna.it/al/articolo?urn=er:assemblealegislativa:legge:2004;16 
Rosen, K., \& Smith, L. (1983). The Price-Adjustment Process for Rental Housing and the Natural Vacancy Rate. The American Economic Review, 73(4), 779-786.

Schwartz, M., \& Wilson, E. Who Can Afford To Live in a Home?: A look at data from the 2006 American Community Survey. US Census Bureau.

The Guardian. (2016, Aprile 2). Recensione di What's Yours Is Mine: Against the Sharing Economy, Tom Slee. Tratto da https://www.theguardian.com/books/2016/apr/02/whats-yours-is-mine-against-the-sharing-economy-tomslee-review

Tomslee. Database Airbnb. Tratto da http://tomslee.net/airbnb-data-collection-get-the-data

Trasporto passeggeri Emilia Romagna. Percorsi autobus notturni. Tratto da https://www.tper.it/o

U.I. Ufficio comunale di statistica - Comune di Bologna. (2017, Luglio). Il mercato immobiliare a Bologna - dati 2016. Tratto da http://www.comune.bologna.it/iperbole/piancont/edilizia/2017/nota\%20immobiliare.pdf

U.I. Ufficio comunale di statistica - Comune di Bologna. Il turismo a Bologna e nell'area metropolitana nel 2015. Tratto da http://www.comune.bologna.it/iperbole/piancont/noterapide/turismo/2015/Turismo_Pres_2015.pdf

U.I. Ufficio comunale di statistica - Comune di Bologna. (2017, Dicembre). I redditi 2015 dichiarati dalle cittadine e dai cittadini di Bologna. Tratto da http://www.comune.bologna.it/iperbole/piancont/Redditi/PRES_REDDITI_2015var50.pdf

Ufficio Provinciale OMI - Territorio della Direzione Provinciale di BOLOGNA. (2016, Novembre 25). Nota territoriale Andamento del mercato immobiliare nel l semestre 2016. Settore residenziale - Bologna. Tratto da Agenzia delle entrate:

https://wwwt.agenziaentrate.gov.it/mt/osservatorio/note\%20territoriali\%20semestrali/note_semestrali_2016 /NT_BO_I_2016_definitiva.pdf 

